\title{
Life after trafficking
}

Citation for published version (APA):

Meshkovska, B. (2018). Life after trafficking: (re)integration processes of women that have been trafficked for the purpose of sexual exploitation in Europe. [Doctoral Thesis, Maastricht University]. Boekenplan. https://doi.org/10.26481/dis.20181122bm

Document status and date:

Published: 01/01/2018

DOI:

10.26481/dis.20181122bm

Document Version:

Publisher's PDF, also known as Version of record

\section{Please check the document version of this publication:}

- A submitted manuscript is the version of the article upon submission and before peer-review. There can be important differences between the submitted version and the official published version of record.

People interested in the research are advised to contact the author for the final version of the publication, or visit the DOI to the publisher's website.

- The final author version and the galley proof are versions of the publication after peer review.

- The final published version features the final layout of the paper including the volume, issue and page numbers.

Link to publication

\footnotetext{
General rights rights.

- You may freely distribute the URL identifying the publication in the public portal. please follow below link for the End User Agreement:

www.umlib.nl/taverne-license

Take down policy

If you believe that this document breaches copyright please contact us at:

repository@maastrichtuniversity.nl

providing details and we will investigate your claim.
}

Copyright and moral rights for the publications made accessible in the public portal are retained by the authors and/or other copyright owners and it is a condition of accessing publications that users recognise and abide by the legal requirements associated with these

- Users may download and print one copy of any publication from the public portal for the purpose of private study or research.

- You may not further distribute the material or use it for any profit-making activity or commercial gain

If the publication is distributed under the terms of Article $25 \mathrm{fa}$ of the Dutch Copyright Act, indicated by the "Taverne" license above, 


\section{Life After Trafficking:}

(re)integration processes of women that have been trafficked for the purpose of sexual exploitation in Europe

\section{Biljana Meshkovska}

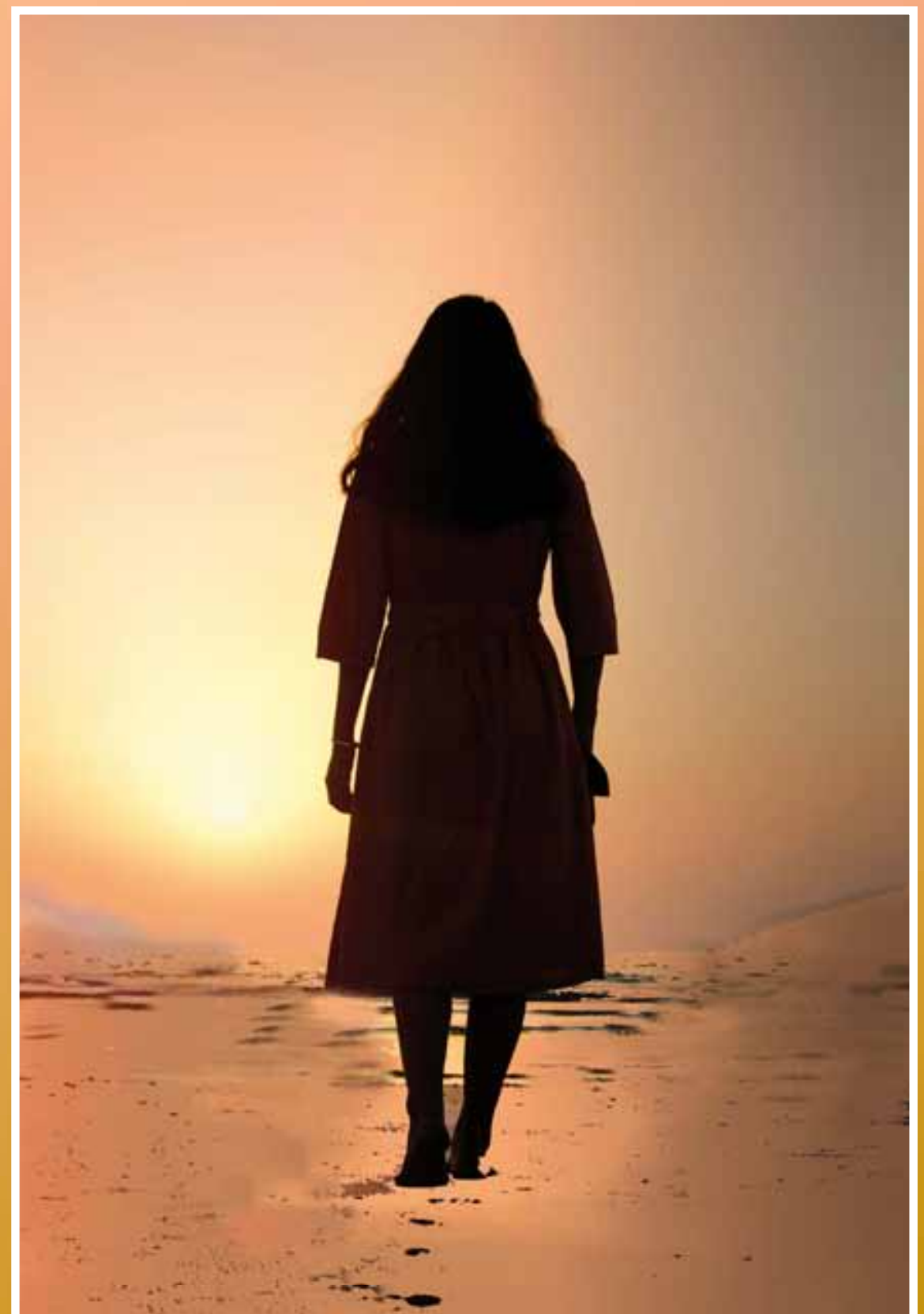





\section{Life after trafficking: (re) integration processes of}

women that have been trafficked for the purpose of sexual exploitation in Europe 
(C) 2018, Biljana Meshkovska

ISBN 9789086664627

Published by Boekenplan, Maastricht www.boekenplan.nl

All rights reserved. No part of this publication may be reproduced, stored in a retrieval system or transmitted in any form, or by any means, electronic, mechanical, photocopying, recording, or otherwise, without the prior permission in writing from the author. 


\section{Life after trafficking: (re) integration processes of women that have been trafficked for the purpose of sexual exploitation in Europe}

\section{DISSERTATION}

to obtain the degree of Doctor at Maastricht University, on the authority of the Rector Magnificus Prof. Dr. Rianne M. Letschert, in accordance with the decision of the Board of Deans, to be defended in public on Thursday 22 November 2018, at 14.00 hours

by

BILJANA MESHKOVSKA 


\title{
PROMOTERS:
}

Prof. Dr. Melissa Siegel

Prof. Dr. Arjan Bos

\author{
ASSESSMENT COMMITTEE: \\ Prof. Dr. Ronald Skeldon (Chair) \\ Prof. Dr. Anja Krumeich \\ Dr. Katie Kuschminder \\ Dr. Marta Bivand Erdal (PRIO, Norway)
}




\section{Content:}

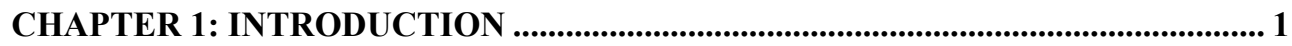

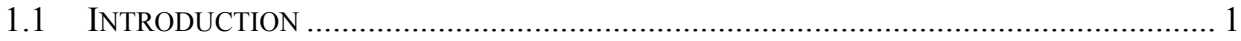

1.2 RESEARCH QUESTION (S) .................................................................................... 4

1.3 REVIEW OF RELEVANT LITERATURE - SEXUAL TRAUMA, VIOLENCE AGAINST WOMEN AND SOME WORDS ON THE IMPORTANCE OF TRUST ………………………................. 5

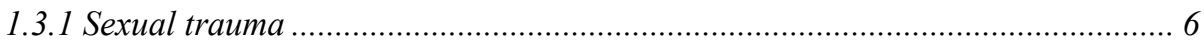

1.3.2 Violence Against Women …………………………….................................. 11

1.3 .3 Trust

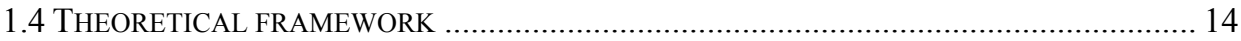

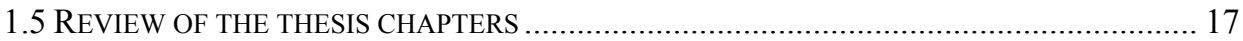

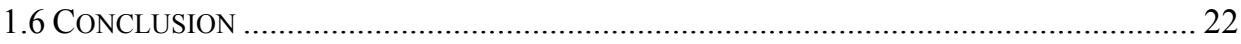

CHAPTER 2: FEMALE SEX TRAFFICKING CONCEPTUAL ISSUES, CURRENT DEBATES, AND FUTURE DIRECTIONS ............................................................... 23

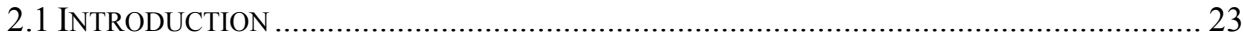

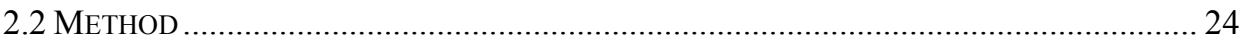

2.3 A DEFINITION OF 'HUMAN TRAFFICKING'............................................................. 25

2.4 CURRENT DEBATES: HUMAN TRAFFICKING VERSUS HUMAN SMUGGLING ...................26

2.5 CURRENT DEBATES ON THE DEFINITION OF FEMALE SEX TRAFFICKING ....................... 28

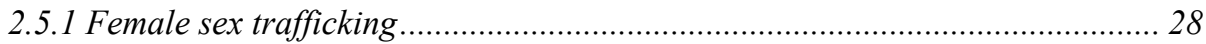

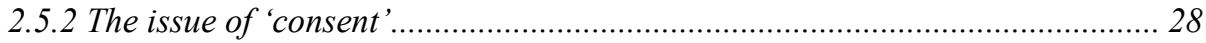

2.5.3 History of the definition of female sex trafficking ........................................... 30

2.6 ESTIMATIONS OF THE NUMBERS OF TRAFFICKED PERSONS .......................................... 32

2.7 FEMALE SEX TRAFFICKING: SECURITY, MIGRATION, AND HUMAN RIGHTS …………... 33

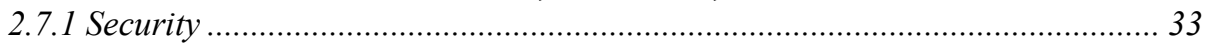

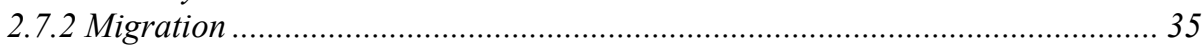

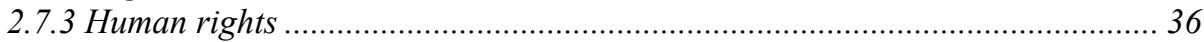

2.8 ACTORS: PERSONS TRAFFICKED, TRAFFICKERS, CLIENTS AND SERVICE PROVIDERS ... 37

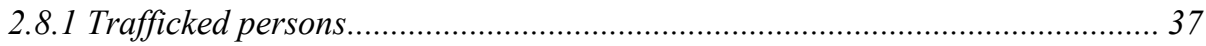

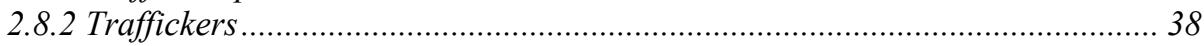

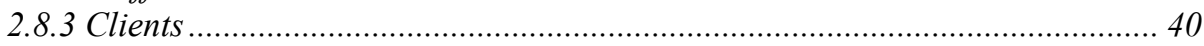

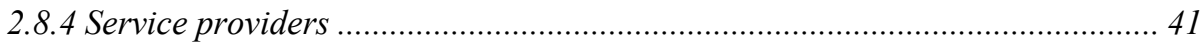

2.9 THE PROCESS OF FEMALE SEX TRAFFICKING ………............................................. 42

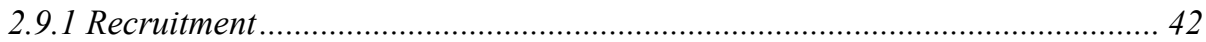

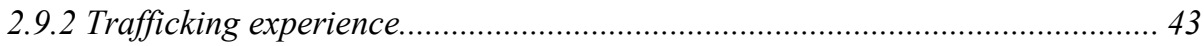

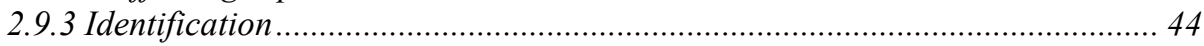

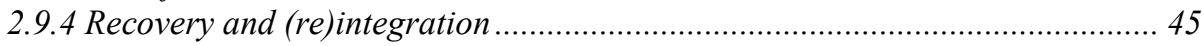

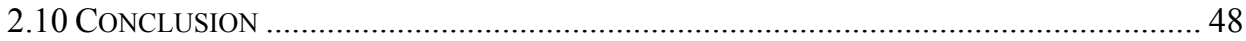




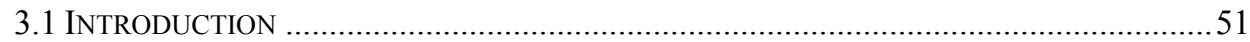

3.2 FORMULATING THE RESEARCH QUESTION ....................................................... 51

3.3 RESEARCH DESIGN: LOCATION, TARGET GROUPS FOR DATA GATHERING, RESEARCH

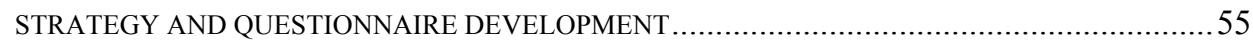

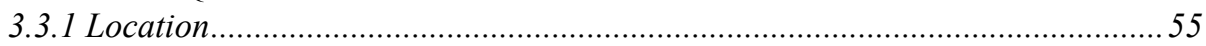

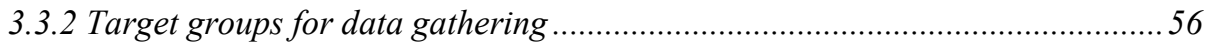

3.3.3 Research strategy and questionnaire development ......................................57

3.4 IMPLEMENTATION: SAMPLING AND OUTREACH, PILOT STUDY, INTERVIEWS AND OTHER

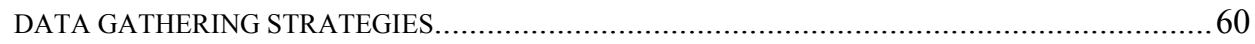

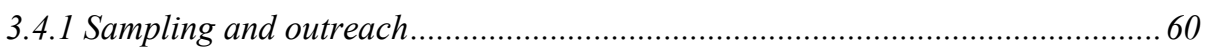

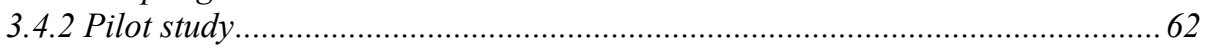

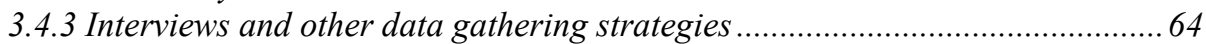

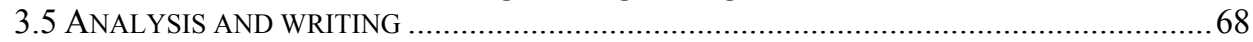

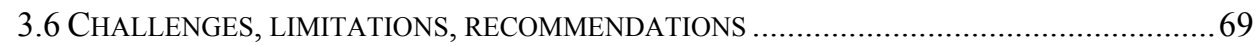

CHAPTER 4: HOW (RE) INTEGRATION SUCCESS AND

(RE) ONCEPTUELINTEGRATION FAILURE IS CONCEPTUALISED IN DIFFERENT CONTEXTS FOR WOMEN TRAFFICKED FOR THE PURPOSE OF

SEXUAL EXPLOITATION

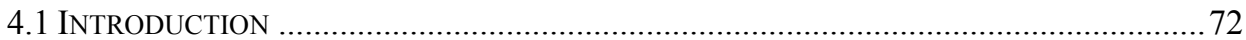

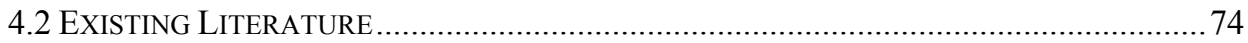

4.2.1 The concept of recovery and the role of resilience ........................................ 74

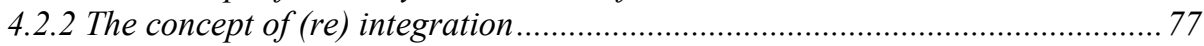

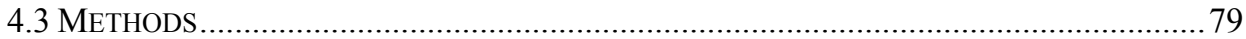

4.4 WHAT ARE THE ELEMENTS OF (RE) INTEGRATION SUCCESS? .................................. 80

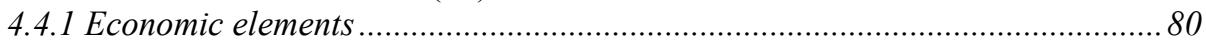

4.4.2 Professional assistance, institutional and legal empowerment ........................ 81

4.4.3 Functioning social relationships .............................................................. 83

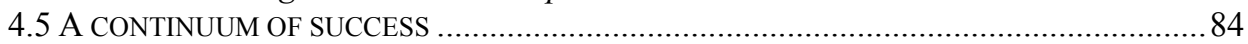

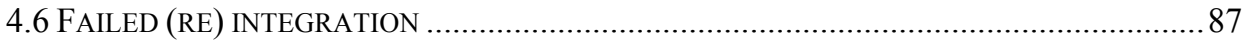

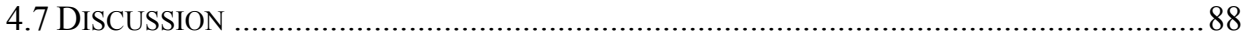

\section{CHAPTER 5: LONG-TERM (RE) INTEGRATION OF PERSONS TRAFFICKED} FOR THE PURPOSE OF SEXUAL EXPLOITATION............................................93

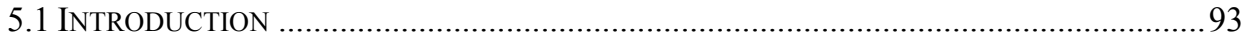

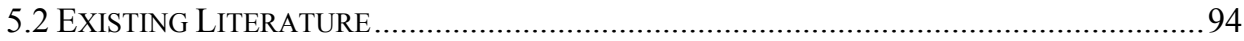

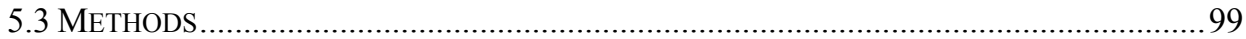

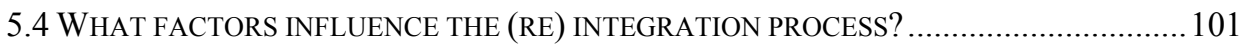

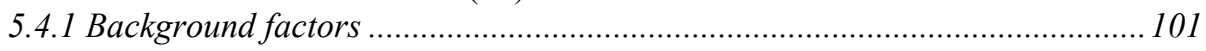

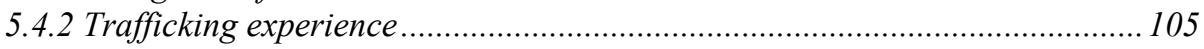

5.4.3 After the trafficking experience: Institutions, NGOs, service providers and their

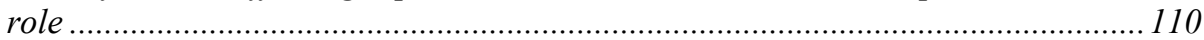

5.4.4 After the trafficking experience: Economic factors ................................... 120

5.4.5 Personal characteristics, challenges, motivations and coping...................... 121

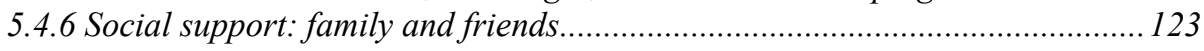




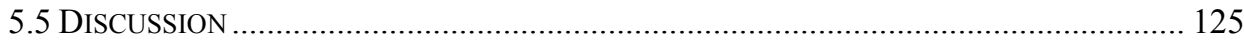

5.5.1 The importance of forming healthy social relationships.............................. 126

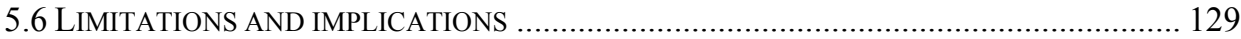

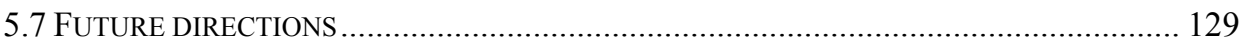

CHAPTER 6: TRAFFICKING OF WOMEN FOR SEXUAL EXPLOITATION IN EUROPE: PROSECUTION, TRIALS AND THEIR IMPACT..................................... 132

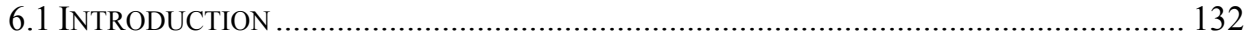

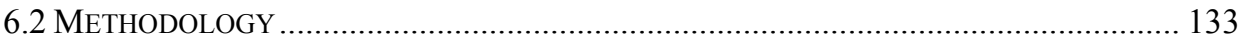

6.3 THE PRACTICE OF THE LAW: THE STORIES OF TRAFFICKED WOMEN AND SERVICE

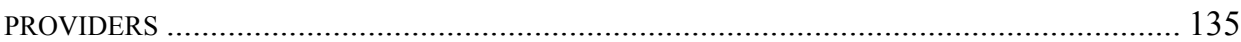

6.3.1 Length of the criminal justice process ...................................................... 135

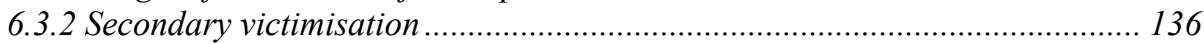

6.3.3 Specialist training and interviewing skills ................................................. 139

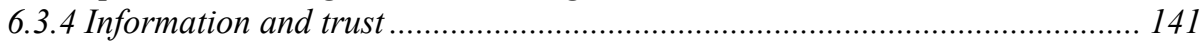

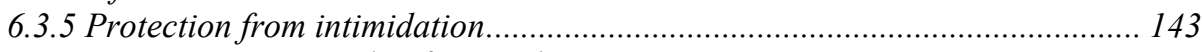

6.3.6 Not just conviction, but financial compensation ............................................. 144

6.3.7 The label 'victim' and the wish to testify................................................... 145

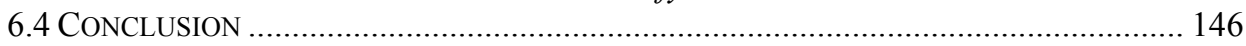

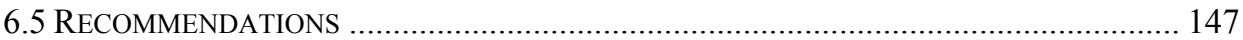

CHAPTER 7: SEX WORK AND TRAFFICKING FOR THE PURPOSE OF SEXUAL EXPLOITATION.......................................................................................... 150

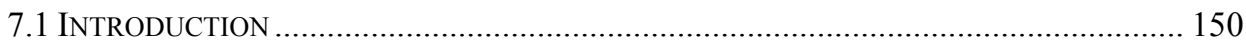

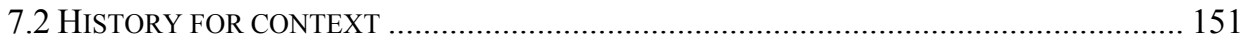

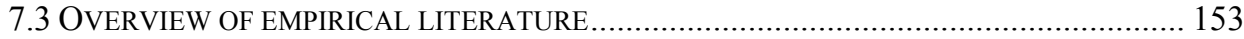

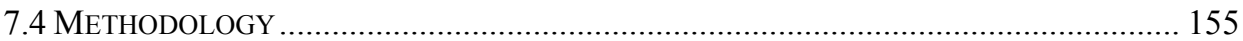

7.5 SEX WORK IN THE CONTEXT OF RECOVERY AND (RE) INTEGRATION ……………........ 157

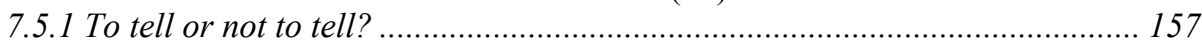

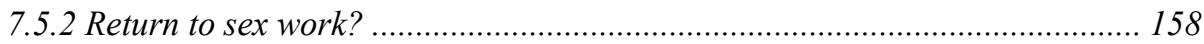

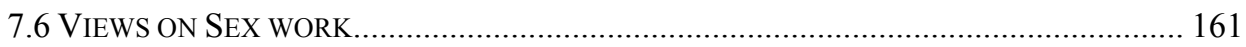

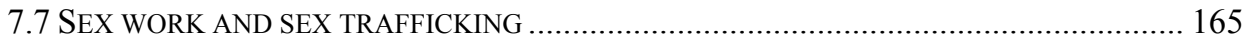

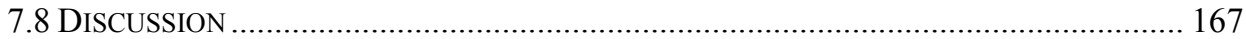

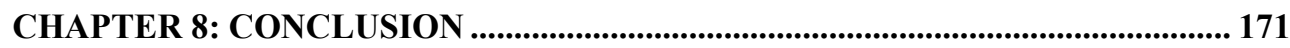

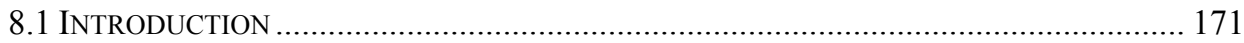

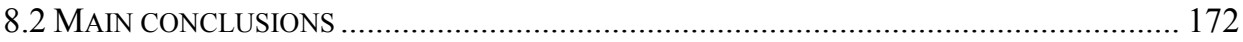

8.2.1 Recovery and (re) integration (Chapter 4) ............................................... 172

8.2.2 Autonomy versus independence (Chapter 4).............................................. 174

8.2.3 Healthy relationships (Chapter 4, Chapter 5) ............................................ 174

8.2.4 Prosecutions and trials (Chapter 6)........................................................ 179

8.2.5 Sex work, trafficking and stigma (Chapter 7) .............................................. 180

8.3 LIMITATIONS AND IMPLICATIONS OF THE RESEARCH ............................................. 182

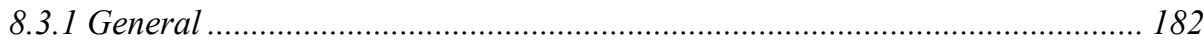

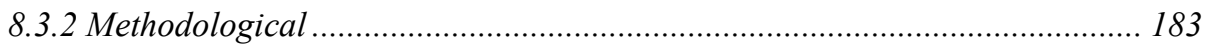

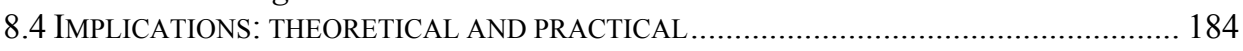

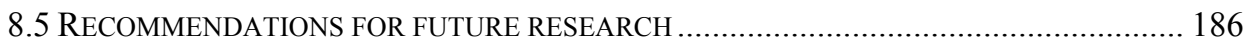


REFERENCES:

ANNEXES

.211

VALORISATION 


\section{Acknowledgements}

In the fall of 2005 I took a course named 'International Trafficking in Persons' taught by Dr. Mohamed Mattar. It was my introduction to the topic of human trafficking. My initial reaction to the issue (which although perhaps naive is nonetheless the very essence of what I felt), was: this cannot be happening in the world today. It is still the way I feel now. It is a thought that stayed with me for many years, and that ultimately led me to my $\mathrm{PhD}$ research. I hope that this thesis is a small contribution to the fight against a crime that regardless of how real, remains unimaginable, and certainly unacceptable.

My research would have been impossible without each individual who agreed to sit down and speak with me. I am in awe of every single survivor I have met. I remember my nervousness before my first interview with a woman who was identified to me as a victim of trafficking. Regardless of where each person was in their life, the strength I believe is required to simply move forward is deserving of admiration and respect. Thank you. Then, there are the persons who dedicate their daily lives to fighting human trafficking. People often say, after a few years in a job like this - helping survivors of such trauma, you have to move on to some other type of work. Many of the people I met strongly contradicted this belief. They have done their job (some for decades), and they would continue to do it with the same energy. Many times I saw tears in the eyes of not only those who have just begun working in this field, but also those that have seen and dealt with the reality of human trafficking for a long time. Thank you for sharing your experiences, and your emotions.

From the very first year of my $\mathrm{PhD}$ research, I have considered myself incredibly fortunate, for I have really had the best supervisors - thank you, Melissa Siegel and Arjan Bos. Your guidance, encouragement and support was invaluable from the very beginning of this journey. I remember the three of us sitting in Melissa's office, before my trip to Albania for the pilot phase of the research, and I remember how excited and curious you both were, as to what I was going to find and bring back. You led me through each bump that came up and helped me find a solution to every problem we crossed throughout these past years. It would be impossible to count the number of drafts each of my chapters has gone through - and thanks to that, I truly 
believe that I have gained the experience expected and needed to be able to earn this degree. But it is not only your professional support for which I am incredibly grateful. I am thankful for the joy and heartfelt congratulations you expressed when I told you I was expecting two babies. It was the beginning of my PhD journey, and I had no doubt I would finish it - but neither did you. Thank you. It has been an honour to be your student.

Thank you Prof. Dr. Maria Kyriakidou, for introducing me to gender studies and academia so many years ago. You have been my very first mentor. It is thanks to you that I started to walk the academic path I am on today.

I want to thank the members of my assessment Committee: Prof. Dr. Ronald Skeldon, Prof. Dr. Anja Krumeich, Dr. Katie Kuschminder and Dr. Marta Bivand Erdal. I additionally want to thank Prof. Dr. Arjen Leerkes, Dr. Sarah Sutterheim, Dr. Julieta Marotta and Dr. Marieke van Houte, who will also be part of my Corona. I am truly privileged to have such scholars read my work, and your constructive criticism has already helped me improve it.

There are friends and colleagues that made my fieldwork throughout the Balkans, Italy and the Netherlands possible. Thank you Marieke Ridder, Fabrice de Kerchove, Erisa Lame, Branka Panic, Nerimana Rifatbegovic, Slavka Kabakchieva and Nenad Sebek for using your professional networks to help me open doors.

Thank you my UNU-Merit/MGSoG family. Mindel van de Laar, you helped me make my first steps when I joined the PhD programme. Since then, you have been a constant support, professionally and personally. It is a pleasure and privilege to keep working with you. Robin Cowan and Tatiana Skripka, thank you for your trust and support throughout these years. Tatiana, you first introduced me to the beauty of qualitative research. I still have and refer to your course slides often, and keep every paper that you recommended on conducting interviews. You also helped me formulate and develop my research question and proposal. Thank you. Eveline in de Break, for me, you will always be the first smiling, friendly face I saw when arriving to UNUMerit/MGSoG. Thank you for your ongoing support - I always know you are just an email away. To my entire class of $2012 \mathrm{PhD}$ fellows - it was a pleasure to spend the first 2 years of this journey with you. Thank you for many wonderful moments Ayo, Thuy, Hasse, Gintare, Carla, Caroline, Tobias, Ralf, Raquel, Stefania, Juan Carlos, Tigist, Sheng, Eriko, Eva, Saba and Martin. To be part of the migration department at UNU-Merit/MGSoG is to 
be part of a family within a family. Thank you Katrin for your encouragement and support throughout the first year of my research (and ever since), as well as your input in my work. I was incredibly lucky to be matched with you as my mentor when I arrived, as I gained not only a wonderful colleague, but also a wonderful friend. Thank you Elaine, Michaella, Ortrun, Jenny, Craig, Simone, Katharina, Carole and Sonja. It is a pleasure to be part of the team. I am also thankful to the people I have met along the way during my years in Maastricht - Sandra, Pico, Andrea, Michael, Ximena, Ilire, Francois, Nevena, Hibret, Ajay, Kristine, Jocelyn and Charlotte. Thank you Mieke and Herman for your ongoing and immediate support, whenever I have needed it. It has been a pleasure working with you.

Thanks to all of you, Maastricht will always stay in my heart.

Thank you my dear paranymphs Iman and Biljana.

Dearest Iman - you are such a wonderful friend. I am very fortunate to be able to keep working with you even now. You will always be the very first person to visit our two little boys in the hospital when they were born. Thank you for your love and support.

Biljana, you are my best friend. It is said that when you meet someone at a very young age, you may be close for a while, but more often than not, you grow apart. I met you when we were both 15 years old, and I still fully remember our very first conversation. We were meant to be - and I have been lucky enough that every single year has brought us closer together. I have been doubly lucky that we are both passionate about, and work in the same field. So you will always be - not only the person I turn to in the most difficult moments - but also the person I ask to proof read my work at $2 \mathrm{am}$ in the morning. Thank you.

The last year and a half of writing and revising my thesis would have been impossible to complete, without having the most wonderful care for my boys. Thank you Elisabeth, Ingvild, Anita, Aksel, Åse Marie, Yashoda, Addis, Ada and Erik. You have the most important and most difficult job of all, raising and taking care of little ones. I am incredibly fortunate, and so grateful.

My family has been an ever-present source of support. Thank you Nikola for lending your legal expertise. Thank you Olivera, Suzana, Aleksandar, Suze, Pece, Dobri, Mima, Cane, Vesela, Vladimir and Goce. 
Thank you Nonno Vincenzo for temporarily giving up your role as mathematician and helping me navigate the Italian anti-trafficking arena. With Nonna Rossana, you have helped our little family move across countries, and helped me finish my research in the process.

Nada, Fanka and Krajna, my dear grandmothers - since I was a little girl, you have shown me what strength, resilience, grace and dignity looks like. Thank you.

On September 272018 I lost my father, Aleksandar. At the moment, it is difficult to imagine a time when it will be possible to accept this loss. But before he left, I was given one last moment of happiness with him. He was the very first person I called and told, that my $\mathrm{PhD}$ thesis has been accepted for defence. I was laughing so much, that he did the same. It was the last time I heard him laugh, and it is the last truly joyful memory I have and will cherish with him. I am grateful for this moment at a time of overwhelming sadness. Thank you my dear aunt Iva, for your love, support and steady voice - you are a piece of him I still have.

Bosiljka - you are my twin. That makes it possible for you to be as loving as you are harsh with me, and it always makes me a better person at the end thank you sis.

Metodija, it is thanks to you that I have the incredible privilege of feeling and knowing that I am never alone. Thank you little brother, for always being there for me. I cannot imagine my life without you.

To my mother Slavka, you are where my strength comes from. The person I have become is a reflection of your efforts. You have always shown me what is most important in life. Words cannot express how thankful I am for being your daughter.

Marco, I would have never reached this present moment, if it were not for your support. You put my work before your own. Made many personal sacrifices and traveled with me throughout Europe to keep me safe. You made it possible that I continue my research, while fully enjoying the most important role of my life - being a mother. Thank you. 
My two boys - Leonardo and Damian. You have filled me with more love and happiness (as well as sheer terror and fear!) than I ever thought possible. From the moment you were born, I am before all else - your mother. You are my life. Thank you for being.

Biljana Meshkovska, October 2018 



\section{Chapter 1: Introduction}

\subsection{Introduction}

A simple google news search using the term 'human trafficking' on any given day, would produce at least 100 news articles published within the last 24 hours. In $2011 \mathrm{CNN}$ launched the Freedom Project, a news initiative aiming to regularly inform the public on the issue of 'modern day slavery', and in that context human trafficking. Most recently, the news agency reported on the active and ongoing slave trade in Libya, which included video footage that shocked the world. The 2030 Sustainable Development Agenda, adopted at the global level in 2015, introduced the goal of ending trafficking of children, as well as the aim of significantly decreasing human trafficking overall, and stopping exploitation and violence against women and girls entirely (UNODC, 2016). National governments are legislating to address the issue, and countless international and national non-governmental organisations have taken up the cause. Human trafficking is at present, a hot topic attracting attention from journalists, policymakers, government and non-government organisations, at the national and international level. However, the question that arises is, where does academia stand in this process and what are the most necessary and urgent ways in which academic work can make a valuable contribution to what is clearly a human rights issue?

Until the United Nations Protocol to Prevent, Suppress, and Punish Trafficking in Persons, Especially Women and Children in 2000, a widely accepted definition of what trafficking is did not exist (UNODC, 2003b). When trafficking was defined, and the Protocol introduced, the primary purpose was to prosecute and punish the traffickers. Practically, the focus was on prosecution, and assistance to those most affected - the persons trafficked was secondary. Since 2000, this has changed and a flurry of organisations at the international and national levels have introduced assistance programs for survivors, some of them making it the sole purpose of their activity and existence. But in this regard in particular, academia has been slow to catch up. 
A more systematic approach and investigation of what the (re) integration ${ }^{1}$ process actually looks like immediately after trafficking, and in the long run is lacking. The initial research in the field of victim (re) integration has been to report on monitoring and evaluation results of assistance programs. Identified impact factors on the (re) integration process are 1) safe and affordable accommodation; 2) legal status; 3) professional and employment opportunities; 4) education and training opportunities; 5) security and safety; 6) healthy social environment; 7) social well-being and positive interpersonal relations; 8) economic situation; 9) physical well-being; 10) mental wellbeing; 11) access to service and opportunities; 12) motivation and commitment to the (re) integration process; 13) legal issues and court proceedings; 14) assistance to secondary beneficiaries (Surtees, 2010). Thus, these would be the indicators looked at, when evaluating the success or failure of (re) integration processes of victims. Scholars have then gone further, to identify some of the challenges to the (re) integration process, such as the social construction of gender, negative community attitudes, physical and emotional health problems, and the lack of victim centred approaches by NGOs (Sharma, 2015). ${ }^{2}$ Factors that help the (re) integration process are family support, increased autonomy and the independence and resilience of the trafficked person (Sharma, 2015). Some research has focused particularly on individual factors, such as the influence of the family on the (re) integration process (Brunovskis \& Surtees, 2012).

Nonetheless, significant questions remain unanswered. When looking at the overall (re) integration process existing research falls short of identifying which factors are of greater and which of lesser importance than others. In addition, it falls short of distinguishing the influencing factors on the (re) integration process in the short term, versus what the needs of the trafficked persons are in the long run, and what preserves or supports the sustainability of their (re) integration. Finally, even when current research focuses on individual factors such as the family, it is the role of the family of origin or family that was created by the trafficked person prior to the experience that is

\footnotetext{
${ }^{1}$ The term (re) integration will be used throughout this thesis, and (re) deliberately placed in parenthesis. Some of the persons captured in this research were never truly integrated into the society in which they lived, prior to the experience of trafficking. They may have lacked basic personal documents such as birth certificates, ID cards and medical cards. Thus, the efforts following the trafficking experience were of integration, rather than reintegration.

${ }^{2}$ A thorough review of research in the area of (re) integration of trafficked persons is given in Chapter 2: Female Sex Trafficking Conceptual Issues, Current Debates, and Future Directions and in Chapter 5: Long-term (re) integration of persons trafficked for the purpose of sexual exploitation.
} 
explored, not so much the role of families that persons may have created after the trafficking experience. It is with these questions in mind, that the research for this thesis began. Thus, this thesis adds to current literature in the following ways: 1) not only looking at factors relevant to the (re) integration process, but also at the dynamics of (re) integration process, and identifying which factors may be more and which less significant; 2) looking at the (re) integration process not only immediately after a trafficking experience, but many years after; 3 ) looking at the significance of the service provider for the (re) integration process of survivors, and the importance of building relationships based on trust; 4) identifying the importance of families created after a trafficking experience for the (re) integration process of survivors; 5) looking at the influence trials against traffickers have on the (re) integration process of survivors and finally; 6) looking at how sex work is addressed in the context of trafficking for the purpose of sexual exploitation, and how persistent stigmatisation of such work fits in within the dynamics of (re) integration.

As $70 \%$ of the persons identified as trafficked are women and girls, and as women and girls are primarily trafficked for the purpose of sexual exploitation, female trafficking for the purpose of sexual exploitation will be the particular focus of this research (UNODC, 2016). The greatest prevalence of female trafficking for the purpose of sexual exploitation is from Central and Southeastern Europe, where the women are trafficked within the region or to Western and Southern Europe (ILO, 2012). Thus, these regions will be the geographical focus of this study.

The purpose of this research is to produce scientific knowledge which can be used to inform assistance programs that are offered to victims of trafficking. And even further to help address the needs of survivors, not just immediately after the trafficking experience, not merely a few years after, but throughout their life.

This introductory chapter will do the following: (1) introduce the research question; (2) review literature that was key in crafting the theoretical framework of this thesis - which is literature on recovery efforts from sexual trauma and recovery of victims of domestic violence as well as some literature on the concept of trust; (3) present the theoretical framework and finally; (4) give a short introduction to each of the individual chapters of the thesis. 


\subsection{Research Question (s)}

As already noted, this research began with the purpose of making a contribution to the identified gap in knowledge in regard to (re) integration processes of women that have been trafficked for the purpose of sexual exploitation, from primarily Central and Southeastern European countries to countries of Western and Southern Europe. The aim is to identify not only the (re) integration dynamics immediately after the trafficking experience, but also in the long run.

Thus, the principal question to be answered is:

\section{What determines successful recovery and (re) integration of women trafficked for the purpose of sexual exploitation, and how do influencing factors interplay, in the short and long run?}

There are several points of the principle research question that should be emphasised. The goal of this research is not merely to identify the factors that influence (re) integration processes, which is what most of the research to date has done. It is to additionally discuss how these factors interact and which are more or less important than others, in the short run, as well as in the long run, the long run being at least 3 years or longer after the trafficking experience. ${ }^{3}$

Thus, this thesis addresses three sub-research questions, which look at different aspects of the principal research question.

(1) What is to be considered a success or failure of the (re) integration process? This question is explored in Chapter 4: How (re) integration success and (re) integration failure is conceptualised in different contexts for women trafficked for the purpose of sexual exploitation. When looking at (re) integration processes, success is measured along the lines of several dimensions, such as whether or not the trafficked person has a safe and stable place to live, her mental and physical well-being and her social support network - to mention just a few. Thus, when we evaluate

\footnotetext{
${ }^{3}$ Monitoring and evaluation research has reported that trafficking victims are usually followed by assistance programs for up to 3 years after the trafficking experience. Thus, longterm in the context of this thesis will be taken to mean the time period after any official monitoring and evaluation efforts by assistance programs have been completed - from 3 years after the trafficking experience, onward.
} 
the success of the (re) integration process, is it absolutely necessary that positive progress is noted along all the relevant dimensions?

(2) What are the influencing factors on the (re) integration process in the short run, and in the long run?

(3) How do these factors interplay, and which may be deemed as more important and which as less important for the overall (re) integration of the trafficked person?

Questions two and three are answered in Chapter 5: Long-term (re) integration of persons trafficked for the purpose of sexual exploitation. The purpose here is not simply to give a list of influencing factors on (re) integration. It is to try to distinguish what seems to be relevant at the very beginning of the (re) integration process, once a person has been identified as a victim of trafficking, and what becomes relevant as time goes on. Are some factors more important than others, in particular for the long-term sustainability of positive progress, in the (re) integration process?

\subsection{Review of relevant literature - sexual trauma, violence against women and some words on the importance of trust}

Trafficking is often linked to the issue of migration. It may be because when thinking of trafficking, many make the mistake of only considering international, and not internal trafficking, associating the process of migration with the dangers of being trafficked. Or of necessarily thinking that for trafficking to have occurred, there must have been some form of 'movement' or 'migration', which is a misconception. In fact, a necessary component of trafficking is an 'action' - such as recruitment, transportation, transfer, harbouring or receipt of persons, rather than a 'movement' or 'migration' (Gallagher, 2010). These issues are more closely analysed and discussed in the literature review chapter of this thesis, Chapter 2: Female Sex Trafficking Conceptual Issues, Current Debates, and Future Directions. Nonetheless, because of this association between migration and trafficking, when addressing (re) integration of trafficked persons, one would first think to draw from literature on (re) integration of migrants, (re) integration of refugees and forced migrants, as well as the (re) integration of ex combatants and child soldiers. At the start of the literature review that was conducted prior to the fieldwork for this thesis, these areas of study were indeed consulted, in order to find a fitting framework through which to address the noted research 
questions. ${ }^{4}$ However, it was found that what these academic fields fail to adequately address is (re) integration from the sexual trauma and abuse that occurs during trafficking for the purpose of sexual exploitation. Thus, it may not be the most fitting literature to give direction for the particular topic of this thesis. This very important, but missing aspect of (re) integration after being trafficked for the purpose of sexual exploitation, is given by literature on recovery from rape, and recovery of women from domestic violence. ${ }^{5}$ It is precisely why the next section will give a brief review of this literature, and point to the theoretical aspects that have given shape to this thesis, in particular in formulating the questionnaires used in the fieldwork. It will also give a short introduction to literature on the issue of trust, as the (re) establishment of trust throughout the process of recovery from sexual trauma and violence is key and will emerge throughout this thesis as an important component of (re) integration after being trafficked for the purpose of sexual exploitation.

\subsubsection{Sexual trauma}

When looking at recovery from rape, several studies adopt the ecological model of trauma recovery, which has been chosen as the most fitting to provide the main theoretical framework to this thesis (Harvey, 1996; Neville \& Heppner, 1999). According to Harvey (1996), through the ecological model, recovery from psychological trauma is understood as a multidimensional phenomenon marked by authority over the remembering

\footnotetext{
${ }^{4}$ For literature on reintegration of migrants refer to: Cassarino, 2010; Constant \& Massey, 2002; Dustmann, 1997; Dustmann, 2003; Dustmann \& Weiss, 2007; King, 2002; Vlase, 2013; Zhang 2013; Diatta \& Mbow, 1999; Ilahi, 1999; Merkle \& Zimmermann, 1992; McCormick \& Wahba, 2003; Rooth \& Saarela, 2007; for literature on reintegration of refugees and forced migrants refer to: Chimni, 2002; Özerdem \& Sofizada, 2006; AbbasiShavazi \& Glazebrook, 2009; Farwell, 2001; Haider, 2009; Zimmermann, 2012; Bascom, 2005; Kibreab, 2002; Cahn, 2006; Gillespie Karl, Peltzer, Malcolm Ma, 2000; Steflja, 2012; Black, 2002; Al-Ali, Black, \& Koser, 2001; Black, Koser, Munk, Atfield, D’Onfrio \& Tiemoko, 2004; Arb, 2001; Koser, 2001; for literature on reintegration of ex-combatants and child soldiers refer to: Watteville, 2001; Annan et al. 2011; Dzinesa, 2007; Veale 2011; Muggah 2007; Brooks 2012; Derluyn et al., 2004; Kohrt, Jordans, 2008; MacMullin and Loughry, 2004; Shepler 2005; Betancourt et. al., 2010; Boothby et al., 2006; Leff, 2008; Stark et al., 2009; Stovel, 2008; Williamson, 2006; Betancourt et al. January 2010; Humphreys and Weinstein, 2007; Knight and Ozerdem, 2004; Paes, 2005; Peters, 2007; Zack-Williams, 2006; Boas and Hatloy, 2008; Wessels, 2004; Specht and Attree, 2006

${ }^{5}$ In this thesis, (re) integration is a distinct concept from recovery, in fact, recovery is one of the dimensions of a wider (re) integration process. These issues will be discussed in detail in Chapter 4: How (re) integration success and (re) integration failure is conceptualised in different contexts for women trafficked for the purpose of sexual exploitation
} 
process, integration of memory and affect, affect tolerance, symptom mastery, self-esteem and self - cohesion, safe attachment, and meaning making. Individual differences in post-traumatic response and recovery are, according to this approach, the result of complex interactions among the person, event, and environmental factors (Harvey, 1996). Neville \& Heppner (1999), through the lens of the ecological model of sexual assault recovery, identify five primary factors that influence recovery, which are the sociocultural context, assault characteristics, personal characteristics, coping responses, and social support systems and interventions. The broader sociocultural context such as a rape prone culture, belong to the macro system, or dimension of recovery. The assault characteristics, such as the relationship to perpetrator and severity of injury, the person variables, such as age, class, previous sexual abuse, pre-rape psychological functioning, race, ethnicity, psychological acculturation, and coping behaviour and responses that are general and culture specific belong to the micro system or dimension of recovery. Finally, social support by family, friends, and generalised others, as well as institutions intervention such as policy or therapy, belong to the meso system, or dimension of recovery. It is precisely these different factors influencing recovery from rape at the micro, meso, and macro level that will be taken as guidelines in sculpting the theoretical frame of this thesis, and in particular in defining the interview questions and areas, for each group of respondents (questions that have to do with micro, meso and macro level factors). The subsequent review of studies will each focus on different factors found to be relevant in recovery from rape, again, at the micro, meso, and macro levels.

Several studies have focused on aspects of the recovery process that are mainly related to the micro dimensions of recovery and the factors influencing it, primarily, although not solely investigating the individual, psychological aspects of the process (Bownes et al., 1991; Burgess \& Homstrom, 1978; Burt \& Katz, 1988; Frazier, 2000; Frazier \& Burnett, 1994; Frazier \& Schauben, 1994; Hill \& Zautra, 1989; Kelland, 2012; Littleton \& Breitkopf, 2006; Regehr et al., 1999; Resick, 1993; Roth et al. 1990; Rothbaum et al. 1992; Smith \& Kelly, 2001; Ullman, 1997). Bownes et al. (1991) investigate the incidence of post-traumatic stress disorder in rape victims in the long-term, finding that there is a $70 \%$ prevalence. Burgess \& Homstrom (1978) study persons 4-6 years post-rape and find that pre-rape stress is not uniform in its effect on recovery, although individual economic hardship, lack of social support, and pre-existing biological, psychological and social problems delay recovery. Burt \& Katz (1988) study women 1-14 
years after rape and find that the five coping strategies of women are avoidance, expressiveness, nervousness/anxiousness, cognitive, and selfdestructiveness. They find that expressive behaviours are the more constructive route to recovery than avoidance coping strategies (Burt \& Katz, 1988).

Frazier (2000) puts emphasis on the importance of issues of self-blame as well as control over the recovery process, stating that self-blame is associated with poorer recovery immediately as well as several years after rape, while feelings of control over the recovery process are most strongly associated to recovery. Frazier \& Burnett (1994) find that coping strategies such as taking precautions, thinking positively, expressing feelings, seeking social support, counselling, and keeping busy have a positive impact on recovery. In a study by Frazier \& Schauben (1999), self-blame is found to be associated with poorer recovery, alongside thinking often about why the rape occurred. Hill \& Zautra (1989) conduct a study of two types of self-blame, characterological and behavioral, and find that they have different effects on adjustment, as they imply different perceptions of control over future aversive events. Kelland (2012) finds that when persons raped express and understand their experience narratively, it can help the recovery process. Littleton \& Breitkopf (2006) study raped university women in the US and find that women who blamed themselves, were vulnerable to reliance on maladaptive avoidance coping and that victims who held negative beliefs about their own worth reported more reliance on avoidance coping. Regehr et al. (1999) focusing on raped women in the US, found that those women with higher levels of self-efficiency, or with perceptions of greater control over the outcome of events in their lives, showed lower rates of depression and post-traumatic stress symptoms. Resick (1993) studied the overall psychological impact of rape on women and found that it caused fear and anxiety, post-traumatic stress disorder, depression, poor self-esteem, social adjustment issues, and sexual dysfunctions. Roth et al. (1990) study the psychological aftermath of sexual assault in university students in the US, focusing on how various aspects of victims' lifetime sexual assault history, their relationships with the assailant mediate post traumatic recovery. They find that persons raped were almost twice as likely as non-victims to meet criteria for psychiatric care (Roth et al., 1990). Rothbaum et al. (1992) focused specifically on the prevalence of PTSD and found that $94 \%$ of women exhibited symptoms immediately after rape, while the percentage dropped to $47 \%$, three months after. 
Smith and Kelly (2001) using the existential-phenomenological method, which aims at uncovering the basic structures of human existence by seeking to describe themes that constitute the experience, study recovery from the perception of the person raped, and identify three stages, starting with reaching out to others, reframing the rape, and finally, redefining the self. Thus, they state that a woman's definition of recovery depends on where she is on her journey of healing. At the beginning of her journey, she defines recovery as resuming her normal routines, talking without crying, freeing herself of obsessive thoughts, and feeling safe again, while further on she redefines recovery as having a new meaning of life through self-love, selfunderstanding, and forgiveness for herself and the rapist (Smith \& Kelly, 2001). Finally, Ullman (1997) compares women sexually assaulted only in adulthood, to women sexually assaulted in both childhood and adulthood, and finds women sexually assaulted in both phases of life reported more psychological symptoms in response to their recent assault and exhibited poorer self-rated recovery and were more likely to blame others. In addition, women still searching for meaning in their victimisation reported poorer recovery, suggesting that such meaning must be found in order to successfully heal from the trauma of sexual victimisation (Ullman, 1997).

Another set of studies have focused on the meso level dimensions that impact recovery from rape (Braithwaite, 2006; Campbell \& Ahrens, 1998; Kinyeki, 2013; Ptacek, 2009; Ullman, 1999). Braithwaite (2006) and Ptacek (2009) note the increasing use of restorative justice in addressing rape recovery. Restorative justice refers to informal, dialogue-based practices, which seek to decrease the role of the state in responding to crime, and increase the involvement of communities in meeting the needs of victims and offenders (Ptacek, 2009). Braithwaite (2006) points out that war often interrupts the 'unthinkableness' of rape, in cultures and societies where until that moment it was unlikely, and points out that rather than prosecution, restorative justice might be more effective in bringing about shame to perpetrators and recovery to persons raped. Ptacek (2009) draws on examples where restorative justice has been used to bring about recovery from rape in the US, Canada, Australia and New Zealand. Drawing community case studies from the US, Campbell \& Ahrens (1998) study coordinated service programs and their effectiveness in promoting positive outcomes for rape victims. They find that these programmes are helpful because they reflect an understanding of the multiple contexts of service delivery and embody that knowledge in services that are consistent with victims' needs (Campbell \& Ahrens, 1998). The model studied proposes three issues of context that explain programme 
effectiveness, which are the context of service delivery, the context of sexual assault in the lives of women, and the context of sexual assault as one of many forms of violence against women (Campbell \& Ahrens, 1998). Kinyeki (2013) studies projects implemented in Sierra Leone, aiming to specifically address the recovery of underage mothers. Finally, Ullman (1999) studies social support offered to persons raped and finds that tangible aid support was more commonly received from formal support providers, negative social reactions were more common for women telling physicians and police, while emotional support from friends was related to better recovery than these social reactions from other support providers. In addition, the study found that if persons raped experienced negative social reactions when disclosing their sexual assault experiences to others, this may contribute to their self-blame and consequently poorer psychological adjustment (Ullman, 1999).

Bletzer \& Koss (2006) approach rape recovery through the macro dimension, by looking at differences and similarities in descriptions of after rape survival, of women coming from different cultural backgrounds. They find that all women mentioned the importance of family and friends, and in the long-term, expressed the desire to forget, exhibited lower self-esteem, difficulty returning to a normal sex life, and not feeling comfortable in social relationships.

It should also be noted that recovery from rape is a complex, long-term process (Burgess \& Holmstrom, 1979; Cohen \& Roth, 1987; Ellis, 1983; Kilpatrick, 1981). Burgess \& Holmstrom (1979) study persons 4-6 years after the rape, and trace effects of the rape on sexual functioning. Cohen $\&$ Roth (1987) study persons 8 years after the rape and identify that the long-term effects of rape depend on demographic characteristics, assault characteristics, post-rape behaviors, coping strategies, and level of functioning. They discover individual differences in severity of symptoms that are related to age, socio-economic status, time since rape, force used during rape, prior history of sexual assault, whether or not the rape was reported, and amount of time it took before confiding in another person (Cohen \& Roth, 1987). Ellis (1983) studies persons at a distance of at least one year post the rape, and finds anger, diminished capacity to enjoy life, hypervigilance to danger, and continued sexual dysfunction. Kilpatrick (1981) examines persons 1 month, 6 months and 1 year post-rape and finds increased levels of anxiety, fearfulness, suspicion, and confusion. 
Finally, some studies point out that there can also be positive effects of the experience of rape, in the long run (Burt \& Katz, 1987; Thompson, 2000). Burt \& Katz (1987) through the study of dimensions of selfconceptualisation, of coping and of self-ascribed change of raped persons, conceptualise and measure how women grow and change in constructive ways, as a consequence of having to cope with rape. Thompson (2000), through the study of women raped 6-40 years ago, notes that $95 \%$ of women reported positive outcomes from recovery from trauma and rape.

\subsubsection{Violence Against Women}

Another group whose recovery process may be of relevance for the (re)integration of trafficked persons is women who are victims of domestic violence. Numerous studies identify and emphasise the health consequences of violence against women (Bennett \& O'Brien, 2007; Fischbach \& Herbert, 1997; Jordan, 2009; Koss et al., 2003).

In a study of women victims of domestic violence in the US, Bennet \& O'Brien (2007) find alcohol and drug abuse prevalence. Fischbach \& Herbert (1997) discover that depression, stress-related syndromes, chemical dependency, substance abuse, and suicide are related to domestic violence. Jordan (2009) finds that the main mental consequences for women that are victims of domestic violence are anxiety, with PTSD, panic, phobia, obsessive compulsive disorder, depression and substance abuse, in addition to low self-esteem and occurrence of self-blame. Koss et al. (2003) also find a prevalence of PTSD among women victims of domestic violence.

Another set of studies focus on some of the services offered to victims of domestic violence (Helfrich \& Aviles, 2001; Hovell, 2006). Hovell (2006) studies police and social service interventions, while Helfrich \& Aviles (2001) note the involvement of occupational therapists in treating victims of domestic violence.

The importance of legal justice, as well as the concept of restorative justice are emphasised by some scholars (Curtis-Fawley \& Daly, 2005; Herman, 2003). Herman (2003) stresses that there are barriers to participation in legal procedures for women, victims of domestic violence, which are psychological, safety related, cultural, and linguistic.

Finally, a significant set of studies puts emphasis on the importance of culture, within the context of recovery from violence, for women (Cribb, 1999; Few, 
2005; Surtees, 2003). Cribb (1999) finds that Samoan women in rural Papa and Christchurch are better at dealing with domestic violence than counterparts in Vaivase Tai, due to individualisation of social relations, changes in economic relations, and cultural responses to the changes, which impact access to services and support for victims of domestic violence.

To conclude, the literature on recovery from rape has pointed to the use of the ecological model of trauma recovery, which addresses factors from the micro, meso, and macro levels. Studies that were reviewed from this field may also focus on factors from just one of the three levels of the model, such as the relevance of self-blame at the micro level, and the social interactions at the meso level. These topics are covered in Chapter 4: How (re) integration success and (re) integration failure is conceptualised in different contexts for women trafficked for the purpose of sexual exploitation and Chapter 5: Longterm (re) integration of persons trafficked for the purpose of sexual exploitation. The literature review on recovery from violence against women points to the relevance of legal justice for the victims, which in turn has also become the primary focus of Chapter 6: Trafficking of Women for Sexual Exploitation in Europe: Prosecution, trials and their impact.

\subsubsection{Trust}

Before moving on to the discussion of the theoretical framework for this thesis, a few words should be said in regard to the concept of trust, which will also emerge as an important theme referenced throughout this thesis, in different chapters, within a varying context in each. The re-building of trust after situations of sexual assault, as well as in the situations of violence against women, reviewed above, is one of the key determinants of recovery. It is crucial in the process of building and re-building of relationships with not only family and friends, after an occurrence of a traumatic event. But it is also imperative for establishing relationships with service providers (such as mental health professionals) who in turn have a significant role in the recovery process of survivors - be it of sexual assault, domestic violence or survivors of trafficking. Thus, a short literature review of how trust becomes relevant in the context of recovery from sexual assault will be given here.

Significant amount of literature on recovery from sexual assault, places emphasis on the importance of the relationship between the survivor and the mental health professional - therapists, psychologist or other type of counsellor (Campbell et al., 2001; Glass et al., 2000; Starzynskia et al., 2017). In some literature the role of the spouse is explored in helping the survivor 
through the process of recovery after a sexual assault (Bilette et al., 2008). In all of these relationships it is the establishment of trust, or re-building of trust that determines if the relationship would be a beneficial one to the overall recovery of the survivor. Thus, Billette et al. find that victims of sexual assault show an improvement in their recovery, from PTSD in particular, when the spouse is also involved in the recovery process (2008). In these cases, the traumatic experience is dealt with not only by the victim, but also with the support of the spouse, within the intimacy of their personal relationship as a couple. Campbell et al. find that in their interactions with different community or institutional systems (offering legal or medical services), following a sexual assault, victims are often subjected to a 'second rape' (2001). This is often due to blaming the victim, for the occurrence of the trauma. It is this blame that causes further erosion of trust from the side of the survivor, and thus prevents the establishment of a relationships between the service providers and the victim that might have been helpful in the recovery process. Glass et al., emphasise the importance of the relationship with the therapist for the recovery of a victim of sexual assault, and the determination of the victim to seek further help, or try to deal with the trauma on their own (2000). Survivors note that it is characteristics of the therapist such as being warm, being kind and patient, and showing empathy and compassion that help in establishing trust between victims and therapist. While boredom, coldness and being impersonal erode trust. Once again, the establishment of trust is seen as key determinant of whether or not the victim continues to seek professional help in dealing with the trauma caused by the sexual assault.

Finally, Starzynskia et al., find that those survivors of sexual assault who have managed to establish trust and have confined to one mental health professional, show greater progress in their recovery, than victims who have failed to establish trust and have met with and disclosed their trauma to multiple health care professionals (2017). Again, in the context of the survivor - mental health professional relationships overviewed in this study, building of trust is crucial, for continuing therapy, and coming to a point in their life when the survivor no longer needs professional help (Starzynskia et al., 2017). Victim blaming and other negative reactions from mental health professionals were found to lead to failure to establish trust, and thus a helpful relationship, which led many survivors to seek assistance from multiple health professionals. In this context, it was found that a turnover of therapists, rather than one long-term therapist who the patient trusts, had a negative influence on the recovery process (Starzynskia et al., 2017). 
The significance of building and re-building trust, in personal relationships with partners, family members as well as with service providers and peers, in the context of recovery and (re) integration following trafficking for the purpose of sexual exploitation, is one of the key findings of this thesis. Consistent with the literature above, it is the establishment of trust, that determines if trafficking victims utilise their relationships with service providers in the context of their recovery, not only in the short, but also in the long run. However, what is additionally significant is to emphasise the behaviours of service providers that may contribute to erosion of trust, again, consistent with the presented literature. Namely, blaming the victim, and in addition to that, trying to exhibit control over her recovery and (re) integration process, has a negative influence on the establishment of trust, and thus on the building of beneficial relationships. As it will be elaborated throughout this thesis - this occurs perhaps even more often to victims of trafficking, than it does to survivors of sexual assault and domestic violence.

\subsection{Theoretical framework}

Having clearly formulated the research question and reviewed some of the literature from a similar field of study that could be helpful in finding the theoretical approach, the next step undertaken is to clearly outline the framework to be used in studying the (re) integration process of women trafficked for the purpose of sexual exploitation. As already mentioned, the most adequate approach that resulted from the literature review undertaken was the use of the ecological model of sexual assault recovery. However, to implement it in the context of human trafficking, it was necessary to go back to the initial ecological model of human development first introduced by Urie Bronfenbrenner in 1979. According to Bronfenbrenner:

"the ecology of human development involves the scientific study of the progressive, mutual accommodation between an active, growing human being and the changing properties of the immediate settings in which the developing person lives, as this process is affected by relations between these settings, and by the larger contexts in which the settings are embedded" (Bronfenbrenner, 1979, p.21)

Going further, he defines the four structures that are the elements of the 'ecological environment': 
"the microsystem is a pattern of (1) activities, (2) roles, and (3) interpersonal relations experienced by the developing person in a given setting with a particular physical and material characteristics" (Bronfenbrenner, 1979, p.22)

"the mesosystem comprises the interrelations among two or more settings in which the developing person actively participates" (Bronfenbrenner, 1979, p.25)

"the ecosystem refers to one or more settings that do not involve the developing person as an active participant, but in which events occur that affect, or are affected by what happens in the setting containing the developing person" (Bronfenbrenner, 1979, p.25)

and finally:

"the macrosystem refers to consistencies, in the form of content of lowerorder systems (micro-, meso-, and exo-) that exits, or could exits, at the level of the subculture or the culture as a whole, along with any belief systems or ideology underlying such consistencies" (Bronfenbrenner, 1979, p.26)

What is of relevance for this thesis, is the occurrence of an 'ecological transition', and 'human development', which would give indications of a successful (re) integration process:

"an ecological transition occurs whenever a person's position in the ecological environment is altered as the result of a change in role, setting or both" (Bronfenbrenner, 1979, p.26)

"human development is the process through which the growing person acquires a more extended differentiated, and valid conception of the ecological environment, and becomes motivated and able to engage in activities that reveal the properties of, sustain, or restructure that environment at levels of similar or greater complexity in form and content" (Bronfenbrenner, 1979, p.27) 


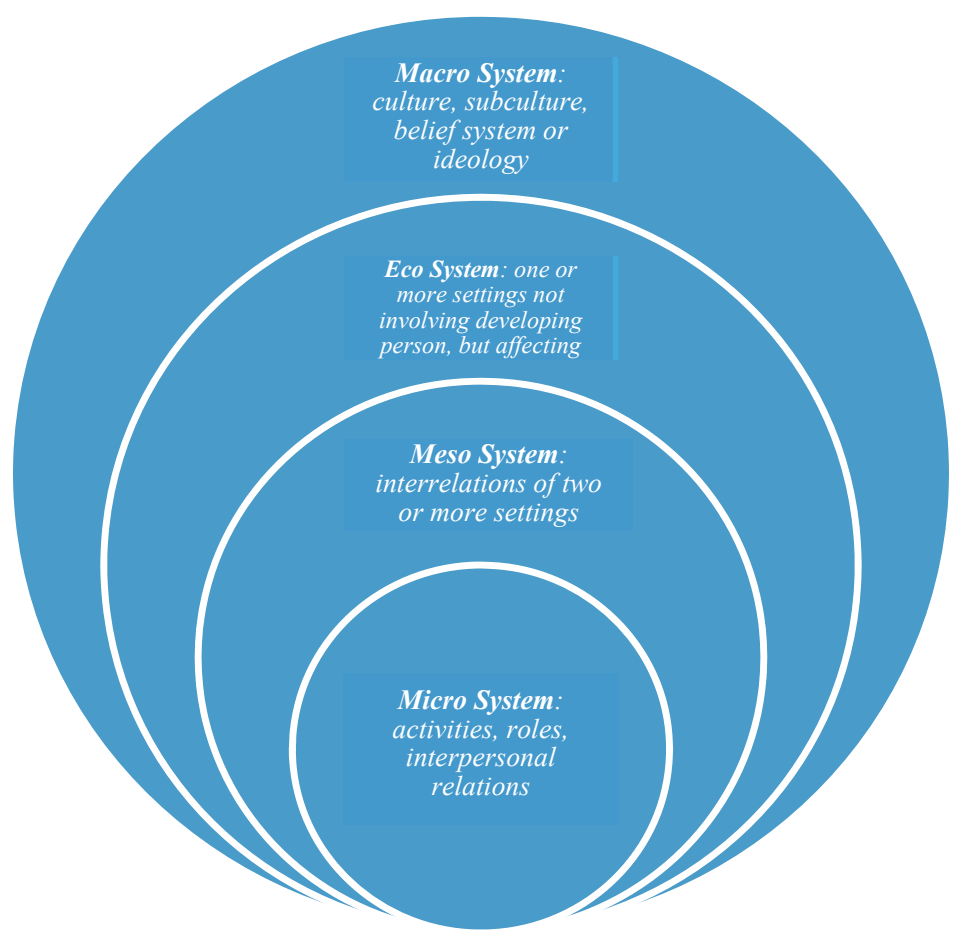

\section{Figure 1: Human Development based on Bronfenbrenner's ecological model}

Thus, one would look at signs of an 'ecological transition' and positive 'human development' as a reflection in the (re) integration process, in the life of a victim of trafficking, through an analysis of the interactions of micro, meso, exo, and macro level factors, through time. However, due to the scope of the study, as well as time and capacity limitations, the primary focus of this thesis is micro and meso level factors.

Overall however, it should be noted that all factors at the micro, meso, and macro level, identified through the work of Brofenbrenner (1979) on the ecological model of human development and Harvey (1996) and Neville \& Heppner (1999) on the ecological model of trauma recovery, have been incorporated in this thesis through the issues addressed in the questionnaire, which are available in their entirety in Annex 2. Additional detail in regard to the way the theoretical framework is used to shape the interview protocols, as well as in regard to the overall Methodology is available in Chapter 3: Methodology. 


\subsection{Review of the thesis chapters}

The final section of this Introduction will give a brief overview of the individual chapters of this thesis.

Table 1: Thesis Chapters

\begin{tabular}{|c|c|c|c|c|}
\hline $\begin{array}{l}\text { Chap- } \\
\text { ter }\end{array}$ & Theme & Key words & Methodology & $\begin{array}{l}\text { Geographic } \\
\text { region }\end{array}$ \\
\hline 2 & $\begin{array}{l}\text { Female Sex Trafficking } \\
\text { Conceptual Issues, Current } \\
\text { Debates, and Future } \\
\text { Directions }\end{array}$ & $\begin{array}{l}\text { human } \\
\text { trafficking, } \\
\text { human } \\
\text { smuggling, } \\
\text { female sex } \\
\text { trafficking, } \\
\text { security, } \\
\text { migration, human } \\
\text { rights, traffickers, } \\
\text { clients, service } \\
\text { providers, } \\
\text { recruitment, } \\
\text { identification, } \\
\text { recovery and } \\
\text { (re) integration }\end{array}$ & Literature review & - \\
\hline 3 & Methodology & $\begin{array}{l}\text { research question, } \\
\text { research design, } \\
\text { location, target } \\
\text { groups, research } \\
\text { strategy, } \\
\text { questionnaire } \\
\text { development, } \\
\text { implementation, } \\
\text { sampling, } \\
\text { outreach, pilot } \\
\text { study, interviews, } \\
\text { data gathering, } \\
\text { analysis, writing, } \\
\text { challenges, } \\
\text { limitations, } \\
\text { recommendations }\end{array}$ & - & $\begin{array}{l}\text { Albania, } \\
\text { Bulgaria, } \\
\text { Bosnia } \\
\text { Herzegovina, } \\
\text { Croatia, } \\
\text { Macedonia, } \\
\text { Montenegro, } \\
\text { Serbia, } \\
\text { Kosovo, } \\
\text { Italy, the } \\
\text { Netherlands }\end{array}$ \\
\hline
\end{tabular}




\begin{tabular}{|c|c|c|c|c|}
\hline $\begin{array}{l}\text { Chap- } \\
\text { ter }\end{array}$ & Theme & Key words & Methodology & $\begin{array}{l}\text { Geographic } \\
\text { region }\end{array}$ \\
\hline 4 & $\begin{array}{l}\text { How (re) integration success } \\
\text { and (re) integration failure is } \\
\text { conceptualised in different } \\
\text { contexts for women } \\
\text { trafficked for the purpose of } \\
\text { sexual exploitation }\end{array}$ & $\begin{array}{l}\text { elements of (re) } \\
\text { integration, (re) } \\
\text { integration } \\
\text { success, } \\
\text { economic } \\
\text { elements, } \\
\text { professional } \\
\text { assistance, } \\
\text { institutional and } \\
\text { legal } \\
\text { empowerment, } \\
\text { social } \\
\text { relationships, } \\
\text { continuum of } \\
\text { success, failed } \\
\text { (re) integration }\end{array}$ & $\begin{array}{l}\text { in - depth } \\
\text { qualitative } \\
\text { research }\end{array}$ & $\begin{array}{l}\text { Albania, } \\
\text { Bulgaria, } \\
\text { Bosnia } \\
\text { Herzegovina, } \\
\text { Montenegro, } \\
\text { Serbia, Italy, } \\
\text { the } \\
\text { Netherlands }\end{array}$ \\
\hline 5 & $\begin{array}{l}\text { Long-term (re) integration of } \\
\text { persons trafficked for the } \\
\text { purpose of sexual } \\
\text { exploitation }\end{array}$ & $\begin{array}{l}\text { factors } \\
\text { influencing (re) } \\
\text { integration, } \\
\text { background } \\
\text { factors, } \\
\text { trafficking } \\
\text { experience, } \\
\text { institutions, } \\
\text { NGOs, service } \\
\text { providers, } \\
\text { economic factors, } \\
\text { personal } \\
\text { characteristics, } \\
\text { challenges, } \\
\text { motivations, } \\
\text { coping, social } \\
\text { support, family, } \\
\text { friends }\end{array}$ & $\begin{array}{l}\text { in - depth } \\
\text { qualitative } \\
\text { research }\end{array}$ & $\begin{array}{l}\text { Albania, } \\
\text { Bulgaria, } \\
\text { Bosnia } \\
\text { Herzegovina, } \\
\text { Croatia, } \\
\text { Macedonia, } \\
\text { Montenegro, } \\
\text { Serbia, } \\
\text { Kosovo, } \\
\text { Italy, the } \\
\text { Netherlands }\end{array}$ \\
\hline 6 & $\begin{array}{l}\text { Trafficking of Women for } \\
\text { Sexual Exploitation in } \\
\text { Europe: Prosecution, trials } \\
\text { and their impact }\end{array}$ & $\begin{array}{l}\text { practice of the } \\
\text { law, length of } \\
\text { criminal justice } \\
\text { process, } \\
\text { secondary } \\
\text { victimisation, } \\
\text { specialist training, } \\
\text { interviewing } \\
\text { skills, }\end{array}$ & $\begin{array}{l}\text { in - depth } \\
\text { qualitative } \\
\text { research }\end{array}$ & $\begin{array}{l}\text { Albania, } \\
\text { Bulgaria, } \\
\text { Bosnia } \\
\text { Herzegovina, } \\
\text { Croatia, } \\
\text { Macedonia, } \\
\text { Montenegro, } \\
\text { Serbia, } \\
\text { Kosovo, }\end{array}$ \\
\hline
\end{tabular}




\begin{tabular}{|c|c|c|c|c|}
\hline $\begin{array}{l}\text { Chap- } \\
\text { ter }\end{array}$ & Theme & Key words & Methodology & $\begin{array}{l}\text { Geographic } \\
\text { region }\end{array}$ \\
\hline & & $\begin{array}{l}\text { information, trust, } \\
\text { protection from } \\
\text { intimidation, } \\
\text { conviction, } \\
\text { financial } \\
\text { compensation, } \\
\text { label 'victim', } \\
\text { wish to testify }\end{array}$ & & $\begin{array}{l}\text { Italy, the } \\
\text { Netherlands }\end{array}$ \\
\hline 7 & $\begin{array}{l}\text { Sex work and trafficking for } \\
\text { the purpose of sexual } \\
\text { exploitation }\end{array}$ & $\begin{array}{l}\text { sex work, return } \\
\text { to sex work, } \\
\text { views on sex } \\
\text { work, sex work } \\
\text { and trafficking for } \\
\text { the purpose of } \\
\text { sexual } \\
\text { exploitation, }\end{array}$ & $\begin{array}{l}\text { in - depth } \\
\text { qualitative } \\
\text { research }\end{array}$ & $\begin{array}{l}\text { Albania, } \\
\text { Bulgaria, } \\
\text { Bosnia } \\
\text { Herzegovina, } \\
\text { Croatia, } \\
\text { Macedonia, } \\
\text { Montenegro, } \\
\text { Serbia, } \\
\text { Kosovo, } \\
\text { Italy, the } \\
\text { Netherlands }\end{array}$ \\
\hline 8 & Conclusion & $\begin{array}{l}\text { limitations and } \\
\text { implications of } \\
\text { the research, } \\
\text { methodological } \\
\text { limitations, } \\
\text { theoretical and } \\
\text { practical } \\
\text { implications, } \\
\text { future research }\end{array}$ & Summary & $\begin{array}{l}\text { Albania, } \\
\text { Bulgaria, } \\
\text { Bosnia } \\
\text { Herzegovina, } \\
\text { Croatia, } \\
\text { Macedonia, } \\
\text { Montenegro, } \\
\text { Serbia, } \\
\text { Kosovo, } \\
\text { Italy, the } \\
\text { Netherlands }\end{array}$ \\
\hline
\end{tabular}




\section{Chapter 2: Female Sex Trafficking Conceptual Issues, Current Debates,}

and Future Directions, is a thorough literature review on the topic of human trafficking more generally, and female trafficking for the purpose of sexual exploitation specifically. Issues such as human trafficking and human smuggling are often confused, and this chapter attempts to clarify this issue. In addition, the most widely accepted definition of what trafficking is, and what specifically, trafficking for the purposes of sexual exploitation is, is given. Trafficking is approached through a variety of fields of study migration, security, and human rights - each of the approaches are reviewed and discussed. Finally, this chapter provides a review on existing literature regarding the different actors involved in the trafficking process - the persons trafficked, the trafficker, and the client. It concludes with literature review sections on the processes or phases of trafficking, namely, recruitment, identification of a person as trafficked, and their (re) integration.

Chapter 3: Methodology, lays out the research strategy undertaken to collect the data for this thesis, analyses, and write-up. It traces the entire process, from formulating the research question, through the numerous literature reviews that were conducted, the rationale behind the choice of target group, type of trafficking to be studied, as well as the way in which the data has been collected. This chapter also gives a thorough description of the experiences of the researcher throughout the pilot study and the interview process, as well as the many challenges faced. It concludes by explaining how the analysis of the data was conducted, and finally, the writing process.

The main body of the thesis consists of Chapters 4 through 7. The topic of (re) integration of persons trafficked for the purpose of sexual exploitation cannot be addressed, without first looking at what success and failure of the (re) integration process may mean in the context of trafficking.

Chapter 4: How (re) integration success and (re) integration failure is conceptualised in different contexts for women trafficked for the purpose of sexual exploitation, looks precisely at this issue. It makes a distinction between recovery and (re) integration, based on literature from the field of psychology, and thereafter looks at the overall (re) integration process, of which recovery is a part, based on empirical research. Based on information gathered from service providers working directly with victims of trafficking, the chapter discusses what can be considered a success of the (re) integration process, or rather the different dimensions of success, and what can be considered a failure of the (re) integration process. 
Chapter 5: Long-term (re) integration of persons trafficked for the purpose of sexual exploitation, based on empirical data gathered through qualitative research looks at the primary factors influencing the process of (re) integration of persons trafficked for the purpose of sexual exploitation. It distinguishes between factors that are relevant in the short run and factors that are important for long-term (re) integration. It also points to factors that may be more significant than others in the process of (re) integration. The structure of the chapter is such that it first looks at the factors relevant to (re) integration starting from the background of the trafficked person, factors that stem from the trafficking experience, from the identification stage, and the immediate aftermath. It then moves on the the present situation of the trafficked person, looking at current economic and social relations as well as the personal characteristics of the trafficked person. The chapter places clear emphasis on factors that are relevant for long-term (re) integration.

The remaining two chapters of this thesis were not conceptualised from the very beginning of this research, but were topics that were identified as relevant for the (re) integration process, based on the literature review conducted, as well as based on the initial fieldwork conducted, in particular the pilot study. It was found that the prosecution of traffickers and the trials against them which usually take a long time do influence the recovery and (re) integration of the victim.

Chapter 6: Trafficking of Women for Sexual Exploitation in Europe: Prosecution, trials and their impact looks into precisely this topic, and explores what the dynamics of the prosecution of the trafficker are, and how they influence the victim. This chapter is slightly different in its nature from the other chapters of this thesis as it is more explorative. As a topic that has been researched very little, much of the findings cannot be directly related to already existing literature. It is meant as a starting point in this field, and attempts to map some of the ways in which prosecution and trials influence the person trafficked, as well as provide some significant policy recommendations.

Finally, Chapter 7: Sex work and trafficking for the purpose of sexual exploitation addresses how the topic of sex work is approached within the context of trafficking for the purpose of sexual exploitation. Women that become victims of trafficking may have knowingly entered sex work, and subsequently were trafficked, as was the case with some respondents interviewed for this research. This chapter explores how they look at sex 
work, following a trafficking experience, and how the service providers that provide assistance programs discuss the issue of sex work in the context of (re) integration. This chapter also touches on issues of ongoing stigmatisation of sex work, and how this may influence whether or not women reveal their trafficking experiences to those around them.

\subsection{Conclusion}

The main purpose of this introduction chapter has been to introduce the topic of the thesis, the central research question, as well as the sub-questions addressed in each of the individual chapters. In addition, it also provides a reference to literature seen as relevant when studying the topic of (re) integration of persons trafficked for the purpose of sexual exploitation - that is, literature on recovery from rape, and recovery of women from domestic violence and on the concept of trust. The review of literature on these topics brought the research to the ecological model of human development, often employed to study recovery from sexual trauma. The model was then presented in its original form, as introduced by Urie Bronfenbrenner in 1979. The original model, looking at factors that influence human development at the micro, meso, and macro level, as well as in conjecture with particular factors at the same levels, as studied in the already mentioned literature on sexual trauma and domestic violence, was then used to give a theoretical frame and reference to this thesis. It was crucial in designing the questionnaires for the fieldwork, which are discussed in greater detail in Chapter 3: Methodology. Finally, the Introduction to this thesis provides an overview of each of the individual thesis chapters. 


\section{Chapter 2: Female Sex Trafficking Conceptual Issues, Current Debates, and Future Directions ${ }^{6}$}

\subsection{Introduction}

Human trafficking is a pressing concern with substantive negative consequences for its victims. It carries relevance for a variety of actors, from the individual looking for employment, or looking to migrate, to the international community responsible for the safety of those targeted. This interest in human trafficking has produced a plethora of information much of which is not based on rigorous empirical research. A lot of the information available is the product of heated debates on issues pertaining to security, human smuggling, female migration, and prostitution. To a lesser extent, information is yielded from lessons learnt in the field. Lastly, and unfortunately, a small portion of the literature is simply a consequence of speculation and marked by inaccuracy. Within the literature on human trafficking, focus has primarily been placed on female sex trafficking, as one of the most severe forms of human trafficking, and thus, it is this type of trafficking that most of research seeks to analyse and understand. A thorough review of the available literature on female sex trafficking that seeks to identify the most urgent issues of concern and disentangle reality from myth is crucial because the existing literature informs policies that seek to tackle human trafficking and assist people that have been affected by human trafficking. The purpose of this article is therefore to provide a comprehensive overview of the main themes involved in female sex trafficking.

This review begins by defining human trafficking and then briefly discusses current issues of contention in human trafficking such as the overlap between trafficking and smuggling and the link between female sex trafficking and sex work. As current issues have their roots in the past, we also include a short historical review that touches on white slavery and the historical origins of sex trafficking. Following that, we review different approaches to sex trafficking including trafficking as a security issue, trafficking as a migration issue, and trafficking as a human rights issue. Thereafter, we provide an

\footnotetext{
${ }^{6}$ An earlier version of this chapter has been published as: Meshkovska, B., Siegel, M., Stutterheim, SE., \& Bos, AE. (2015). Female sex trafficking: Conceptual issues, current debates, and future directions. Journal of Sex Research, 52(4), 380-95. doi:10.1080/00224499.2014.1002126
} 
overview of the main actors that play a role in sex trafficking processes, namely trafficked women, traffickers, clients, and the central service provider for persons trafficked. After the overview of the main actors, the focus shifts to the trafficking process including methods of recruitment and characteristics of the trafficking process. We then discuss methods of identification as well as processes of recovery and (re) integration of victims.

\subsection{Method}

The main themes of this Chapter were determined and outlined as a result of a preliminary review of literature on human trafficking in general, and sex trafficking in particular. Thereafter, a thorough literature review of the English language literature was conducted in order to identify the most relevant literature within each theme. Google Scholar, PsychInfo, Web of Science, ProQuest, Scopus and Medline were the main databases used. The primary key word employed was 'human trafficking'. It yielded 35300 hits in Google Scholar, 2668 hits in ProQuest, 16651 hits in Web of Science, 359 in Scopus and 143 hits in Medline. Each were subsequently sorted by relevance. The same methodology was employed for the following additional key words: 'protocol', 'definition', 'sexual exploitation', 'sex trafficking', 'smuggling', 'prostitution', 'sex work', 'history', 'security', 'migration', 'human rights', 'trafficked person', 'actor', 'victim', 'survivor', 'trafficker', 'demand', 'customer', 'client', 'service provider', 'recruitment', 'trafficking experience', 'identification', 'recovery', 'integration' and 'reintegration'. Based on the relevance of the title and the contents of the abstract to the identified themes, a final selection of 241 sources: articles (206), books (21) and reports (14) was made, all of which were read in detail. Of the final 241, 138 (113 articles, 7 books, 14 reports, 2 official documents, 1 discussion paper, 1 handbook) were selected for inclusion in the review. Literature was chosen for inclusion based on the relevance to the topic covering the major themes identified as important points of discussion for this chapter. Papers covering conceptual issues were selected on the basis of how current and relevant the topic addressed was to ongoing debates in the field, how critical and well argued the approach, and the clarity of writing. Empirical papers were chosen on the basis of a sound and transparent methodology, as well as quality analyses. The final number of selected literature also includes grey literature, such as reports, commissioned or published by international organisations, government organisations or research institutes. Grey literature was included when the contents were deemed important for understanding a current trend in the field, such as when discussing the scope and geographical 
distribution of female sex trafficking, as well as practices of recovery and (re) integration. Finally, relevant treaties, reports, and documents from the United Nations (UN), United Nations Office for Drugs and Crime (UNODC), UN Gift, European Union (EU), International Organization of Migration (IOM), International Labor Organization (ILO) and the United States (US) State Department were included. It is important to note that the issue of human trafficking has been sculpted through internationally debated and UN initiated documents. Thus, this review pays attention to these documents, particularly as they pertain to definitions of human trafficking and sex trafficking. The overall time period that the selected literature covers ranges from January $1^{\text {st }}, 1999$ to April $30^{\text {th }}, 2014$. We opted not to look further back in time because the definition of human trafficking as it is today was introduced in 2000 .

\subsection{A definition of 'human trafficking'}

The contemporary and commonly accepted definition of human trafficking was introduced in 2000 by the United Nations Protocol to Prevent, Suppress, and Punish Trafficking in Persons, Especially Women and Children. According to this United Nations Protocol, human trafficking is

"the recruitment, transportation, transfer, harboring or receipt of persons, by means of threat or use of force or other forms of coercion, of abduction, of fraud, of deception, of the abuse of power or of a position of vulnerability or of the giving or receiving of payments or benefits to achieve the consent of a person having control over another person, for the purpose of exploitation. Exploitation includes, at a minimum, the exploitation of the prostitution of others or other forms of sexual exploitation, forced labour or services, slavery or practices similar to slavery, servitude or the removal of organs" (Protocol to Prevent, Suppress, and Punish Trafficking in Persons to the UN Convention against Transnational Organized Crime, 2003, p. 2)

The Protocol continues with Article 3 (b), which states that consent is irrelevant, should any of the means noted be used. Finally, Article 3 concludes by noting that means are irrelevant when the person is a child, or below the age of 18 .

Based on the UN definition, Gallagher (2010) distinguished three elements which must be present for trafficking to have occurred: "(1) ACTION: recruitment, transportation, transfer, harboring or receipt of persons; (2) 
MEANS: threat or use of force or other forms of coercion, of abduction, of fraud, of deception, of the abuse of power or of a position of vulnerability or of the giving or receiving of payments or benefits to achieve the consent of a person having control over another person and (3) PURPOSE: exploitation" (Gallagher, 2010, p. 29).

It should be noted that the UN Protocol speaks not only of women and girls, but men, women, and children (UNODC, 2013). In addition, the inclusion of the segment 'harbouring or receipt of persons' makes clear that human trafficking is not only a process, but also pertains to the maintenance of a situation of 'exploitation' (Gallagher, 2010; UNODC, 2013). Finally, it also indicates that trafficking occurs not only across, but also within borders (UNODC, 2013).

Despite its comprehensive nature, the UN Protocol leaves numerous questions unanswered. From its inception onward, these questions have given rise to debate. One issue of contention is the interchangeability between trafficking and smuggling in practice. Another is the issue of 'consent' in the context of sex trafficking.

\subsection{Current debates: Human trafficking versus human smuggling}

Shortly after introducing the Anti-trafficking Protocol, the UN also introduced a protocol against the smuggling of migrants by land, sea, and air as a supplement to the United Nations Convention Against Transnational Organized Crime. In this protocol, the smuggling of migrants was defined as:

"the procurement, in order to obtain, directly or indirectly, a financial or other material benefit, of the illegal entry of a person into a State Party of which the person is not a national or a permanent resident" (UNODC, 2003, p. 2)

Seeing as the purpose of this protocol is to 'prevent and combat the smuggling of migrants' (UNODC, 2003), we can contend that smuggling differs from trafficking in that smuggling requires the crossing of borders. In contrast, trafficking can happen within the confines of state borders. In addition, smuggled migrants, unlike trafficked persons, can be deported to their country of origin immediately upon identification, without the possibility of being offered temporary or permanent residence as is the case for some trafficked persons in certain countries and situations (Gallagher, 2010). 
Although these distinctions may be clear on paper, in practice, it is hard to distinguish trafficking from smuggling (Aronowitz, 2001; O'Connell Davidson, 2013; Skilbrei \& Tveit, 2008). This is particularly when one takes into consideration differences in definitions of smuggling introduced at national levels (Aronowitz, 2001; O'Connell Davidson, 2013). According to Aronowitz (2001), the fact that both groups are considered 'irregular migrants' makes them vulnerable to exploitation - although it should be noted that trafficked persons are not always 'irregular migrants' while smuggled persons are. Thus, smuggling can easily become trafficking.

O'Connell Davidson (2013) reviewed how some policy makers, nongovernmental organisation (NGO) activists, and academics see trafficking and smuggling. She makes two distinctions: the first being that trafficking can be a process, whereas smuggling is a one-time transaction, and the second being that trafficking involves 'coercion and deception' while smuggling is 'voluntary'. However, drawing on numerous examples from practice, she discusses how smuggled migrants often, in an effort to finance their migration, acquire debts so great that they are required to accept working conditions that could very much be labeled as exploitative. At the same time, she argues that there are also trafficking situations in which physical violence is not present, trafficked persons are fully aware of the type work they will be conducting, and debts can be paid off in a short timeframe of one to three years (O'Connell Davidson, 2013). Thus, the question remains: wherein does the difference between smuggling and trafficking lie? And if we cannot provide a clear answer to this question, but want to remain true to the identified categories and definitions of 'trafficked' and 'smuggled', how do those, in practice situations, responsible for determining the difference decide how to allocate persons that could be deemed as trafficked and smuggled? In such decision-making moments, is it the interests of the state or the interests of the individual that are primarily taken into consideration? Unfortunately, in looking at how border policies are implemented, it becomes evident that the interests of the state takes precedence over the interests of the individual. 


\subsection{Current debates on the definition of female sex trafficking}

\subsubsection{Female sex trafficking}

In understanding and defining female sex trafficking, which is essentially the trafficking of women for purpose of sexual exploitation, we often rely heavily on the definition of human trafficking outlined above. However, it should be noted that a clear definition of 'sexual exploitation' is not yet available. In the literature, various authors provide variations of the definition. For instance, Segrave, Milivojevic, and Pickering (2009) define sex trafficking as "trafficking of women into sexual servitude" (p. 1).

\subsubsection{The issue of 'consent'}

According to those present during the drafting process (Doezema, 2005; Doezema, 2010; Gallagher, 2001; Raymond, 2002) of the Protocol to Prevent, Suppress, and Punish Trafficking in Persons, Especially Women and Children, the primary issue of debate was the issue of 'consent' and, particularly, "whether non-coerced, adult migrant prostitution should be included in the definition of trafficking" (Gallagher, 2001, p. 984). Thus, the definition of trafficking became a battleground between those that consider it possible for sex work to be a voluntary choice and those that consider prostitution to always be forced. The trafficking definition was thus to decide if the international community would lean in favour of sex work or against prostitution.

The first camp promoting and protecting sex worker rights gathered under the Human Rights Caucus and was led by the Global Alliance Against Trafficking in Women (GAATW; Doezema, 2005). The second, proabolitionist camp was the International Human Rights Network, primarily led by the Coalition Against Trafficking in Women International (CATW; Raymond, 2002). According to CATW, "there are different degrees, levels and extent of coercion, abuse and violence perpetrated against any woman or child at any particular time, which is critical to the individual. But all women who are in the sex industry are violated and sexually exploited" (O'Connor \& Healy, 2006, p. 5).

For those belonging to the Human Rights Caucus, or in general, those that believe sex work can be a choice, deeming consent irrelevant would mean infantilizing and disempowering the choices that adults, or more specifically, women make (Doezema, 1999; Doezema, 2005; Doezema, 2010). This camp claims that such a tendency is already evident in the field of human trafficking 
and that this is particularly apparent when we look at images of the ideal female victim - a woman who is deprived of her ability to make choices and completely lacks agency. In addition, such deprivation of the possibility to consent would clearly take away the option to work freely for many that are currently sex workers by choice.

For the International Human Rights Network, or in general, those that consider all prostitution to be violence against women, recognising consent as a possibility in the context of human trafficking would be mean making a distinction between "deserving and undeserving" victims (Raymond, 2002, p. 494). It would put the burden of proving a situation of trafficking on the victim and provide a possible out for the trafficker, who can then claim that the person consented to the work (Raymond, 2002). According to this camp, 'sex work' should not be considered as just any type of labour as it most often involves some kind of harm to those that practice it, and the dangers of that work are very different than the dangers of other kinds of labour. Finally, sex work cannot and should not be considered empowering for women given that it is driven by male demand (Dempsey, 2010; Jeffreys, 2009; Miriam, 2005).

So how did this battle play out in the context of the Protocol? As Gallagher (2010) explains, the protocol does not include the phrase 'irrespective of the consent of the person'. However, it does leave place for consent to be invalidated if any of the other means noted, such as 'coercion, of abduction, of fraud, of deception' are present (Gallagher, 2010, p. 27). Additionally, Hoyle, Bosworth, and Dempsey (2011) have argued that including terms that have not been clearly defined, such as 'abuse of power or of a position of vulnerability' makes it possible to make consent irrelevant in the future.

Today, it is clear that the clash between the two camps is still being fought, perhaps in particular because the definition of female sex trafficking does not clearly favor one side or the other. Thus, countries such as the US, labeled by some as 'neo-abolitionist', are in a position to continue to carry out the battle through, for example, finances (Soderlund, 2005) and, in that context, USAID grants funds only to those anti-trafficking organisations that are clearly against prostitution (Overs \& Hawkins, 2011; Soderlund, 2005). Overs and Hawkins (2011) point out that this kind of behaviour is especially damaging to sex workers as the NGOs that were crucial in offering health services to sex workers now have limited access to funding. Such a policy, as well as a push to adopt an abolitionist stance within the Protocol, gained substantial ground during the presidential terms of George W. Bush, who linked the 
moral grounds of his war on terror with a war against trafficking (Soderlund, 2005; Weitzer, 2007).

Studies have also been published showing that the legalisation of prostitution is tied to persistent or increased levels of female sex trafficking (Huisman \& Kleemans, 2014; Kenyon \& Schanz, 2014; Marinova \& James, 2012). It is, however, important to recognise that quantitative studies seeking to show that legalisation of prostitution leads to increased trafficking are questionable, as the numerical data available on prostitution as well as trafficking is far from reliable or complete (Cho, Dreyer, \& Neumayer, 2013; Jakobsson \& Kotsadam, 2013).

Munro (2006) notes that despite the friendliness of some legislative systems to sex work, stigma still persists. Thus, stigma, in combination with the pressure to register as a sex worker, as in the Netherlands, may be the real culprit for sex workers' choice to remain 'illegal' and thus vulnerable to exploitation, even though they have the option of legality (Outshoorn, 2012). Hubbard, Matthews, and Scoular (2008) compared a number of legal systems as they pertain to sex work and prostitution and concluded that they may all have similar outcomes in that most drive visible sex work and prostitution away from the streets and eyes of society underground where all involved are, once again, vulnerable to abuse.

Finally, there are those that propose a disengagement with this long-standing debate in favour of sex work and against prostitution (Barnhart, 2009; O'Connell Davidson, 2006; Wagenaar \& Altink, 2012). Some scholars note that the underlying factor in all discussions is the presence or absence of exploitation. Thus, they claim that efforts should focus on defining exploitation and distinguishing different levels of exploitation, as these should resolve many of the current debates and struggles played out in discussion surrounding the issue of sex trafficking.

It can be argued that the unrelenting ties between sex work, prostitution, and trafficking have their roots in the past, which is why they are not easy to overcome.

\subsubsection{History of the definition of female sex trafficking}

It is currently quite common to come across references to female sex trafficking as modern-day slavery in reports by not only the media, international organisations, and governments, but also in the academic 
literature. One quite apparent reason for this is that human trafficking has its roots in white slavery. White slavery is understood as the "procurement by force, deceit, or drugs, of a white woman or girl against her will, for prostitution" (Doezema, 1999, p. 25). As Silvia Scarpa (2008) notes, at the start of the twentieth century, numerous international conventions were adopted due to the sudden rise of white slavery and what is noticeable in the conventions, over time, is shifts in terminology from 'white slave traffic' to 'traffic in women and children'. Finally, in the immediate predecessor of the 2000 Protocol, namely the Convention for the Suppression of the Traffic in Persons and the Exploitation of the Prostitution of Others, which was introduced in 1949, we see "race, gender and age neutral terminology" (Scarpa, 2008, p. 52).

The pressure to address the issue of white slavery, and the manner in which it was addressed, primarily by asking for the abolition rather than the regulation of prostitution, was the result of efforts on the part of feminist abolitionists, led by Josephine Butler (Scarpa, 2008). Thus, the 1949 Convention had an abolitionist stance toward prostitution, which some say is why there was such a low number of signatories at the international level (Gallagher, 2010; Scarpa, 2008). It should be noted that today's CATW has its roots in the same abolitionist movement of the 1900s.

Another reason why trafficking is often equated with slavery can be found in the definitions of both. Bales (2012) defines contemporary slavery as "the total control of one person by another for the purpose of economic exploitation", noting that although slavery in the past was a matter of 'legal ownership', "today it is a matter of having full control over a person through the use of violence" (Bales, 2012, p. 5-6). Similarly, the previously mentioned definition of trafficking refers to 'slavery or practices similar to slavery' as forms of exploitation, or as end goals of the trafficking process, thus one could contend that 'slavery' is contained within the definition of trafficking (Gallagher, 2010).

It should be noted that there are scholars who claim that there is no data showing that white slavery was indeed a reality (Doezema, 1999). In fact, it seems to have coincided, as does present day human trafficking, with a period of feminisation of migration (Doezema, 1999). These scholars claim that the movement of women in order to work as prostitutes has in the past, as today, often functioned to create a 'moral panic', resulting in the creation of the myth of 'white slavery' (Doezema, 1999). Thus, white slavery has served as a tool 
for abolitionist to take away the ability of women to choose sex work, and thus restore the 'moral order' of society (Bernstein, 2007; Doezema, 1999). These same scholars claim that it is in the interest of such groups to use strong dichotomies such as freedom and slavery, which translate into images of the 'whore' or the 'innocent victim', in order to attract and secure public sympathy. In fact, some claim that if we look critically at the way in which trafficked persons are classified today, we will see that women who are not deemed 'pure' enough or who have not shown remorse for their choices are not given the status of being trafficked. In addition, the stories that tend to reach the public and that are contained in the reports of international organisations are those of the beautiful victim that was unaware that she would be working as a prostitute, that repents and that wants help in order to reform but practices no agency of her own and is left to the will of benevolent saviours (O'Brien, Carpenter, \& Hayes, 2013; Saunders, 2005). In countries such as the US, abolitionists are often joined by religious groups that seek to confine sex to the institution of marriage (Bernstein, 2007; Soderlund, 2005).

Taking all the above into consideration, regardless of the profile of the 'victim', the nature of exploitation, or the scope of the issue, trafficking serves well to justify strict border controls and restrictions on migration, especially for female migrants. The current data on female sex trafficking, the problems that accompany estimations, as well as approaches to sex trafficking through the prism of security, migration, and human rights are discussed in the next section of this chapter.

\subsection{Estimations of the numbers of trafficked persons}

The most commonly quoted estimation of the number of persons trafficked, provided by the International Labor Organization (ILO), claims that 20.9 million persons are currently subjected to forced labour, of which $55 \%$ are women and girls, and 22\% are forced into sexual exploitation (ILO, 2012). The ILO uses the 'capture-recapture' method to produce the estimation, which has received significant criticism. Additional estimations are those provided by the Trafficking in Persons Report, published yearly by the US State Department, which claims that 600,000-800,000 men, women, and children are subject to human trafficking annually, or a total of 27 million, a number also given by Kevin Bales, an expert on modern day slavery (Bales, 2012; US Department of State, 2013). 
However, all the above noted numbers are only estimations. The United Nations Office of Drugs and Crime (UNODC) has provided the most comprehensive data on cases identified as human trafficking in a report published in 2012. According to the UNODC, information was collected from throughout the world, primarily from government bodies, on 55,000 cases of trafficked persons. Of the noted cases, $59 \%$ were women and $58 \%$ of all cases are situations of sexual exploitation. It should be noted that sexual exploitation is the dominant form of human trafficking in Europe, Central Asia, and the Americas. Thus, the same report cautions that the predominance of this form of human trafficking may be a result of better identification methods in these particular parts of the world, or of this particular form of trafficking.

\subsection{Female sex trafficking: Security, migration, and human rights}

There are three dominant approaches to the issue of sex trafficking, and their primary relevance is not only what they offer in terms of theoretical discussions on trafficking but also the policies they suggest. Thus, trafficking is most commonly seen as an issue of state security, an issue of migration, or an issue of human rights. Each of the noted approaches, their policy implications, and criticism received, are discussed below.

\subsubsection{Security}

Gallagher (2010), a prominent human rights lawyer affiliated with the UN, recalls the outrage of colleagues when the issue of human trafficking was removed from the auspices of the United Nations human rights system and given to the United Nations Office for Drugs and Crime. However, in retrospect, she notes that it was the right decision because the Protocol would never have received the attention it has had, had it stayed solely a human rights issue (Gallagher, 2010). Thus, the primary driver for pushing through the UN Protocol was state security. Human trafficking was pegged as a transnational, organised criminal activity, and, as such, a threat to each country and its people. Not only was it claimed that transnational criminal organisations were the primary culprits of the crime, it was also noted that profits from trafficking activities are used to finance conflicts and terrorism in various parts of the world (Goodey, 2003; Shelley, 2010).

The language used in the Protocol, when speaking of criminalisation versus assistance and protection, also makes obvious that the protocol is primarily a tool used for the protection of state security (Fitzpatrick, 2002; Gallagher, 
2010; Lobasz, 2009). Article 5 of the Protocol asks for the criminalisation of human trafficking, noting that "each state party shall adopt" all measures deemed necessary to establish trafficking as a criminal offence (Protocol to Prevent, Suppress, and Punish Trafficking in Persons, 2003, p. 2). However, Article 6, which addresses assistance and protection of persons trafficked and asks for the provision of housing, counseling, and information; medical, psychological, material assistance; as well as employment, educational and training opportunities, adopts a softer language, noting "each state party shall consider" providing the noted services to persons trafficked (Protocol to Prevent, Suppress, and Punish Trafficking in Persons, 2003, p. 3).

A criminalisation and security approach to human trafficking has brought about primarily two types of policy responses from states: (1) stricter border controls; and (2) raids of establishments where supposedly trafficked women are kept (Goodey, 2003). Both approaches have been the target of harsh criticism.

Researchers note that the consequence of strict border controls is that it serves to push individuals wanting to migrate into the hands of actors that could potentially take advantage of them (Andrijasevic, 2003; Chapkis, 2003; Schaeffer-Grabiel, 2010; Skeldon, 2000; Väyrynen, 2003; Vijeyarasa, 2013). In other words, strict border controls may serve to make women migrants, especially sex migrants, thus women migrating with the primary purpose of seeking employment in the sex industry, more vulnerable to trafficking.

On the other hand, raids, which are rarely used at present, are criticised for often being employed as a method for curbing prostitution rather than addressing sex trafficking. In addition, reports have been recorded where the women that have been 'saved' through such methods have then been abused by their 'rescuers' (Amar, 2009; Lindstrom, 2004).

Interestingly, the security approach not only identifies traffickers as dangerous, but also the person who is trafficked is identified as potentially dangerous for the receiving country and its people. Aradau (2004) elaborates in detail on how the international politics of 'pity' inspired by idealised versions of the pure, innocent, and unknowing victims of trafficking have been transformed into politics of 'risk'. In other words, women who have been abused can be seen as potential future abusers. Thus, the safest strategy for receiving countries is to ship these women back to where they came from. It is in this way that repatriation and deportation have been justified to the 
public (Muftić, 2013; Segrave et al., 2009). Thus, these women, while considered to deserve pity, are nonetheless construed as different and as the 'other' (Berman, 2003; Goodey, 2003; Todres, 2009). They are seen as practicing devious activities and having questionable morals, and are therefore, best kept outside of borders (Aradau, 2004). In the name of protecting its citizens, the state regains control and creates a new purpose for its existence (Berman, 2003).

\subsubsection{Migration}

The current international emphasis on the issue of human trafficking is said to have coincided with a period of increased female migration. Economic crisis, conflict, a sense of adventure, and curiosity have prompted many women to cross borders in search of economic means for themselves or their families, or simply in search for a better life (Mai, 2013). However, it has been suggested that it may be this increased willingness to migrate that makes women more vulnerable to abuse and that feeds the supply side of human trafficking. Researchers have attempted to strengthen this claim by linking high migratory movements and developed migrant networks in destination countries to a rise of human trafficking (Cho, 2013). It is these links that have then prompted policies such as tighter border controls and campaigns that seek to discourage the migration of women (Nieuwenhuys \& Pécoud, 2007).

Once again, strict border controls and anti-trafficking campaigns have received strong criticism. Some claim that the issue of human trafficking is grossly exaggerated so that it can be used as a tool that would curb female migration, in particular migration for the purpose of sex work (Haynes, 2004; Pickering \& Ham, 2014). Information campaigns launched by actors such as the International Organization of Migration subscribe to the image of the innocent, unknowing female victim versus the evil male trafficker (Andrijasevic, 2007). Trafficking is thus often equated with prostitution and potential female migrants are given the impression that, should they attempt to cross borders, they may end up being sex slaves. Anecdotes told in prevention and information leaflets speak of women applying for jobs in the service sector but unknowingly and unwillingly ending up in prostitution. Thus, once again, this touches on the issue of consent. The stories of women who knowingly seek employment in the sex sector are not found in information pamphlets and campaigns (Andrijasevic, 2007).

Furthermore, this group is not always visible. The extent to which these women are labeled as 'trafficked' or as an 'illegal immigrant' often depends 
on the needs of the country in question at the moment. For instance, Brennan (2014) points out that, when Argentina wanted to show that it was making progress in its anti-trafficking policies by increasing its victim identification efforts, it grouped together trafficked persons with immigrant sex workers and labeled all as trafficked (Brennan, 2014). In contrast, Brazil does not make a distinction between trafficking and prostitution. Thus, all women involved in the sex industry in Spain coming from Brazil, whether they are persons trafficked or immigrant sex workers, are labeled as trafficked (Piscitelli, 2012).

As some suggest, one way to resolve the current crisis would be to not solely focus on trafficking, but also on migration rights and citizenship rights of illegal immigrants and illegal sex workers, as well as labour rights for all sex workers (Anderson \& Andrijasevic, 2008; Andrijasevic, Aradau, Huysmans, $\&$ Squire, 2012; Chapkis, 2003; Skeldon, 2000). In other words, it is argued that attempts should be made toward recognising sex work as a form of labour and making it possible for women to safely perform it either in their home countries or through working visas across borders. This, it is claimed, would constitute a true approach to trafficking through migration and would address the current vulnerability that illegal migration creates for sex workers (Agustín, 2006).

\subsubsection{Human rights}

The common framing of sex trafficking as modern-day slavery and an emphasis on the pervasiveness of violence has made it increasingly possible to approach the issue of female sex trafficking through the lens of human rights (Saunders, 2005). The reasoning is simple: As basic human rights are broken by the practice of sex trafficking, the human rights approach is crucial for addressing the needs of those affected.

The Protocol, and the already noted language surrounding the topics of assistance and protection of victims, was seen as a major failure for the human rights camp (Fitzpatrick, 2002). However, despite the soft language, in practice the basic services that should be available to those trafficked are being offered. In some cases, this happens through the state; in others, through international and nongovernmental organisations. Several of the noted provisions are the non-discriminatory application of anti-trafficking law, noncriminalisation of persons trafficked, possibility for financial compensation, protection of persons trafficked, possibility to stay in receiving countries, and 
some basic services such as medical and mental health care and shelter (Jordan, 2002).

It is worth noting that several researchers have emphasised that not all violations that happen within a trafficking experience are necessarily human rights violations. There are situations that fall within the current definition of trafficking but are not a violation of human rights. These are more likely to be violations of labour rights. One such example is when a sex worker shares her fee with an agent (Munro, 2008, Shamir, 2012). Therefore, the framing of the issue of trafficking as always and solely a matter of human rights should not always be taken for granted.

Finally, as an addition to the three approaches described above, some researchers have noted a need to look at some of the systemic factors that may be responsible for the existence of female sex trafficking (Hua \& Nigorizawa, 2010; Limoncelli, 2009; Todres, 2005; Wong, Holroyd, Chan, Griffiths, \& Bingham, 2008). Instead of discussing consent and whether or not sex work can be voluntary, we should work towards creating situations in which women always have choices and opportunities, thus efforts should be made to eliminate situations in which sex work is an economic imperative. Therefore, it is claimed, policies should address poverty, educational opportunities for women, and gender discrimination (Hua \& Nigorizawa, 2010; Limoncelli, 2009; Todres, 2005; Wong et al., 2008).

\subsection{Actors: Persons trafficked, traffickers, clients and service providers}

The following section of this chapter focuses on the primary actors involved in sex trafficking. However, although a description of the prevailing profiles of each actor as found in the literature are given, it should be noted that the characteristics of each actor are not exhaustive. There is no such thing as a 'typical' profile of a person trafficked, trafficker, client, or service provider.

\subsubsection{Trafficked persons}

The primary targets of sex trafficking are women or as Vijeyarasa (2013) eloquently states, "trafficking is gendered, and the gender is female" (p. 1024). Poverty and being unemployed prior to the trafficking experience are common characteristics attributed to trafficked women (Acharya, 2010; Hughes, 2000; Silverman et al., 2006; Vindhya \& Dev, 2011). Women trafficked are often described as having low levels of education and as coming from difficult family situations where they suffered some form of physical or 
sexual violence (Acharya, 2010; Hughes, 2000; Reid, 2012; Silverman et al., 2006; Vindhya \& Dev, 2011; Zimmerman, Kiss, Hossain, \& Watts, 2009). However, although these characteristics are frequently mentioned, they are by no means the rule. There are many women that do not come from a situation of extreme poverty but simply ended up being trafficked when looking for a way to make more money by accepting sex work (Pajnik, 2013; Stoecker, 2000; Surtees, 2004). Some women simply desire a lifestyle that they have heard about from other female migrants that work abroad or a wealthy lifestyle as presented in magazines and on television shows such as Brazilian soap operas and so-called Cinderella stories (Vijeyarasa, 2012; Vijeyarasa, 2013). In these cases, the women tend to be relatively welleducated but dissatisfied by the material benefits of their professions (Pajnik, 2012; Stoecker, 2000; Surtees, 2004).

\subsubsection{Traffickers}

One of the primary reasons that anti-trafficking legislation was enacted at the international level was because perpetrators of human trafficking have been framed as highly organised, transnational criminal groups and networks (Shelley, 2010; Surtees, 2008a). For example, focusing on traffickers in South East Europe, Surtees (2008a) noted that these organised crime groups working across countries comprise members of various nationalities and ethnicities. However, extensive recent research has failed to make connections between transnational organised crime and human trafficking (Chin \& Finckenauer, 2011; Piscitelli, 2012; Zhang, 2011).

Pimps are commonly mentioned as actors responsible for sex trafficking (Chin \& Finckenauer, 2011; Marcus, Horning, Curtis, Sanson, \& Thompson, 2014; Verhoeven, van Gestel, de Jong, \& Kleemans, 2013; Zhang, 2011). Because sex trafficking can be based on an intimate relationship between the woman and her pimp, sex trafficking can be seen as a crime of a 'relational nature' (Verhoeven et al., 2013). In this context, the man initially establishes his status as a boyfriend and subsequently controls the woman through emotions, intimidation, or violence. Overall, however, it is noted that, in most cases, pimps are not connected with organised crime. In fact, the daily activities of a pimp appear to be highly individualised and thus do not require the involvement of organised crime. On the other side, the activities of a pimp are not very profitable, thus making that role unattractive for most organised crime groups (Zhang, 2011). 
It should be noted that not only men, but also women, can be pimps. Studies find female pimps to be predominantly in high-end escort services (Zhang, 2011), but they can also be found in other roles. One such role is that of 'recruiter'. Women can act as recruiters, in many cases while they themselves are sex workers or victims of sex trafficking. Female recruiters have also been found to frequently work together with a man who, after the recruitment by the women, is responsible for the transportation (Surtees, 2008a). It has also been noted that women that have managed to create a good living for themselves as sex workers sometimes offer novices startup loans. For example, Piscitelli (2012) reports that women from Brazil that have established themselves as sex workers in Spain sometimes offer to pay for the trip and startup of other Brazilian women that knowingly want to perform sex work. Naturally, interest rates on such loans tend to be exuberant and the irony of these situations is that, although these established sex workers could be considered traffickers according to Brazilian law, they are considered victims of trafficking themselves because they are already sex workers in a foreign country (Piscitelli, 2012).

Perhaps the most notorious role of female traffickers is that of the 'madam' (Chin \& Finckenauer, 2011; Leman \& Janssens, 2013; Mancuso, 2014; Siegel, 2012). The most common case noted is that of the Nigerian madams, who are usually former sex workers themselves that have moved upward and created their own establishments which recruit and employ other women. Women from Nigeria are then either sold by their family or ask for a loan from the madam themselves in order to leave Nigeria and work as prostitutes throughout Europe. Interestingly, the way in which the madam ensures repayment of debts is by binding the woman and her family through voodoo and juju rituals. A recent study, performing a social network analysis to determine the role of different actors in a trafficking case in Italy, found the role of the madam to be central to the trafficking process (Mancuso, 2014).

Chin and Finckenauer (2011) note a number of additional actors that are often attributed the role of trafficker. Actors that loan money to a woman or her family in order to enable her to travel to another country and conduct sex work are usually referred to as 'agents', and are considered to have ownership of the woman until she repays her debt. Some other actors noted are the 'jockeys' that are responsible for the physical transfer of the woman to her place of work, 'fake husbands' that marry women that want to travel to other countries for sex work, and 'mommies' who introduce women to clients, in 
addition to 'escort agency owners' and 'brothel owners' (Chin \& Finckenauer, 2011).

Finally, it should be noted that in many cases traffickers justify their participation in trafficking through a variety of arguments. This is known as a process of 'neutralization' (Copley, 2014). Copley (2014) charts different strategies by which this is done. Some traffickers attempt to minimise the exploitation by seeing themselves as helping rather than hurting the person trafficked. Others dehumanise the victim, seeing them as objects rather than people. Another strategy entails shifting the blame for the occurrence of trafficking to, for instance, corruption. A final strategy outlined by Copley (2014) is one in which traffickers call upon the necessity of trafficking in certain situations, such as the need to supply women to soldiers during a war.

\subsubsection{Clients}

The actor least known within the process of sex trafficking is the client. It is extremely hard to distinguish between clients of voluntary sex workers and clients of trafficked persons. A study performed by Anderson and O'Connell Davidson (2003) focusing on clients of sex workers in Denmark, Thailand, India, and Italy has shed some light on how clients view the purchase of sexual services from a person that is trafficked. This study found that those clients that viewed prostitutes as objects and paying for sex as a trade, tended to like exercising control in these situations and were thus more likely to seek out sex workers in vulnerable situations (Anderson \& O'Connell Davidson, 2003). In another study by Todres (2009), the author claimed that discrimination based on race, ethnicity, or class is the driving force behind certain men purchasing sex from certain groups of women. Preference for trafficked women comes about because the women are seen as an 'other' and thus looked down upon, and their vulnerability and submissiveness is seen as an attractive trait (Todres, 2009).

Other literature has explored specific kinds of clients. Some studies have placed the onus for increased trafficking on soldiers and peacekeepers who have, as a group, increased the demand for sex workers (Allred, 2006; Haynes, 2004). The claim is that, when the supply of voluntary sex workers insufficiently meets demand, the rest is provided through trafficking. An aggravating factor in such situations is that these actors usually have immunity in the country where they are stationed and thus cannot be held accountable for purchasing sex from victims of trafficking despite adequate legislation being in place (Allred, 2006). 
The final circumstance that generates a discussion of clients and the demand side of sex trafficking is sporting events. Football world cups, cricket tournaments, super bowl matches, and Olympic games have all been identified, primarily by international organisations, governments and the media, as occasions that potentially increase the demand for sex workers, and thus, sex trafficking victims. However, increases in the incidence of sex trafficking in these situations has been widely debated (Bonthuys, 2012; Deering et al., 2012; Gould, 2010). Roe-Sepowitz, Gallagher, and Hickle (2014), in their study of sex trafficking and sex worker demand during the 2014 Super Bowl, demonstrated an increase in number of advertisements offering sexual services around the time of the Super Bowl. However, they failed to show any fluctuation or possible increase in demand during the actual Super Bowl, as opposed to any other point in time (Roe-Senowitz et. al, 2014). Both Bonthuys (2012) and Gould (2011) focused on the Football World Cup that took place in 2010 in South Africa and found that media reports, and anti-trafficking campaigns at the time, all estimated that 40,000 persons were trafficked for the event. Both authors also found the same unsubstantiated number of estimated trafficked persons in media on the 2006 World Cup in Germany. Gould (2011) also examined whether there indeed were reports of an increase in human trafficking prior to and following the 2010 Football World Cup and found no such increases. Naturally, international organisations and governments credit the lack of increase in human trafficking cases during sporting events to the implemented antitrafficking campaigns (Gould, 2011). It should be noted that the lack of evidence on the impact sporting events can have on human trafficking is potentially because it is difficult to properly assess changes in numbers of people trafficked in general and that the short period of time allotted to such sporting events makes this even more difficult.

\subsubsection{Service providers}

The final actors relevant to the issue of sex trafficking are service providers. Unfortunately, there is a scarcity of research focusing on the various service providers, such as the social workers, psychologists, lawyers, police, and doctors who in some way have had contact with victims of trafficking, which tends to occur after identification.

However, a recent study has noted social workers as the most important service providers for victims of trafficking (Busch-Armendariz, Nsonwu, \& Heffron., 2014). In fact, this study identified the social worker as the central figure for the trafficking victim for three main reasons. First, social workers 
are the main coordinators of all interactions not only between other service providers and a trafficked person but also between all other service providers involved in a case. Second, social workers are usually the ones responsible for building and establishing trust with victims. Third, social workers are usually found to be culturally sensitive and well trained to deal with diverse groups of victims and thus are, through the application of the ecological model, the most helpful people for victims immediately after identification.

\subsection{The process of female sex trafficking}

This section places the different actors within the process of sex trafficking. It thus discusses common methods for recruitment used by traffickers, common characteristics of the trafficking experience, common means by which trafficked persons are identified, and common processes of recovery and (re)integration that follow identification.

\subsubsection{Recruitment}

There are two relevant aspects of the recruitment process, namely the person or persons doing the recruiting and the method of recruitment. The method of recruitment is visibly connected to the vulnerability of the target.

One commonly mentioned recruiter and method of recruitment is the pimp who gains the trust and thus controls the woman by becoming her boyfriend and, in certain situations, by offering marriage (Crawford \& Kaufman, 2008; Deshpande \& Nour, 2013; Di Tommasso, Shima, Strøm, \& Bettio, 2009; Hom \& Woods, 2013; Verhoeven et al., 2013; Vindhya \& Dev, 2011). The vulnerability of the person trafficked in these situations may be the need for affection and love (Hom \& Woods, 2013). Preying on this vulnerability, the pimp establishes his role as a boyfriend (Verhoeven et al., 2013) and shortly thereafter asks the woman to perform sex work in order to support the couple materially. The pimp often uses intimidation and violence in order to control the woman (Verhoeven et al., 2013). This process has been termed 'pimp enculturation' by Hom and Woods (2013).

Another commonly used method of recruitment is one in which a woman is offered employment. With this method, the trafficker preys upon the woman's economic vulnerability or, alternatively, simply plays into her desire to migrate (Crawford \& Kaufman, 2008; Di Tommasso et al., 2009; Hughes, 2000; Jones, Engstrom, Hilliard \& Sungakawan, 2011; Silverman et al., 2007; Surtees, 2008a; Vindhya \& Dev, 2011). In these situations, the recruiter may 
be someone the woman knows personally, such as a friend, or the recruiter could be a stranger (Di Tommasso et al., 2009). Additionally, this method of recruitment can occur through the internet, TV, or through newspaper advertisements (Di Tommasso et al., 2009; Hughes, 2000). In these cases, the employment offered is usually babysitting, serving as a hostess in the food and beverage industry, or dancing (Di Tommasso et al., 2009; Hughes, 2000; Surtees, 2008a). In some cases, recruiters are open about the fact that the employment on offer is sex work (Di Tommasso et al., 2009; Jones et al., 2011; Molland 2010; Molland, 2011; Piscitelli, 2012), and sometimes the recruiter is a sex worker herself (Molland, 2011; Piscitelli, 2012). Consequently, when women knowingly accept sex work, the form of exploitation generally pertains to working conditions (Jones et al., 2011) or to the division of profits between the sex worker and the recruiter (Molland, 2010). For example, Jones et al. (2011) outlined how women from Thailand were offered transfer to Japan in order to work in the sex industry but upon arrival, they were informed of debt incurred, their documentation was taken away from them, and they had no control over the number of customers or type of sex to be provided until the debt was paid off.

\subsubsection{Trafficking experience}

Although each trafficking experience has its own unique characteristics, some commonalities have been reported. For example, Zimmerman and colleagues (2009), in their study that focused on 192 women receiving post-trafficking services in Belgium, Bulgaria, the Czech Republic, Italy, Moldova, Ukraine, and the United Kingdom, found that the trafficking experience lasted from 1 day to 3 months for $33.2 \%$ of the women, 3 to 6 months for $19.3 \%, 6$ months to a year for $19.8 \%$ of the women, and longer than a year for $18.8 \%$ of the women. Oram et al. (2012), in their study that explored 120 cases of trafficked women returned to Moldova, found that $67.5 \%$ were trafficked for more than 6 months and $27.5 \%$ were trafficked for more than a year. Vindhya \& Dev's (2011) study that looked at 78 case studies of trafficked women in India, found an average duration of the trafficking experience to be two years.

In terms of working conditions, studies have reported a lack of choice with regard to client selection, types of sexual services offered, and use of condoms (Di Tommaso et al., 2009; Jones et al., 2011). Also, Di Tommaso and colleagues (2009) established that the average sex trafficked woman in their study worked every day of the week, 13 hours a day, with typically five clients per day (Di Tommaso et al., 2009). Acharya (2010) documents more than eight clients per day for $43 \%$ of the victims in this study. Additionally, studies 
have noted that sex trafficked women often have limited or no freedom of movement and limited or no access to medical care (Di Tommaso et al., 2009; Jones et al., 2011; Zimmerman et al., 2008). Physical and sexual violence during the trafficking experience have also been reported frequently and can include rape, gang rape, being beaten with objects, and being burnt with cigarettes (Acharya, 2010; Crawford \& Kaufman, 2008; Di Tommaso et al., 2009; Jones et al., 2011; Zimmerman et al., 2008).

The trafficking experience has also been found to be accompanied by physical and mental health symptoms that can be short-term or can have long-term health consequences. Physical symptoms documented include headaches, tiredness, dizziness, back, stomach and pelvic pain, tooth pain, a loss of appetite, physical injuries, unsafe abortions, and sexually transmitted infections including HIV (Collins, Goldenberg, Burke, Bojorquez-Chapela, Silverman \& Strathdee, 2013; Konstantopoulos et al., 2013; Oram et al., 2012; Wirth, Tchetgen, Silverman \& Murray, 2013; Zimmerman et al., 2008). Decker, McCauley, Phuengsamran, Janyam, and Silverman (2011), in their study conducted with female sex workers in Thailand, found those who entered sex work through trafficking to be at greater risk for sexual violence upon initiation and violence in the work place, and to be more likely to have abortions and not use condoms or use them inconsistently, which increases their vulnerability to HIV infection.

Mental health symptoms reported include post-traumatic stress disorder, depression, and anxiety (Abas et al., 2013; Chudakov, Ilan, Belmaker, \& Cwikel, 2002; Konstantopoulos et al., 2013; Zimmerman et al., 2008). Sex trafficking victims are also more likely than victims of other types of trafficking to use alcohol and drugs (Lyneham \& Larsen, 2013).

\subsubsection{Identification}

Successful identification has been defined as a "situation in which antitrafficking professionals were able to identify individuals as trafficked (or potentially trafficked) in an appropriate, sensitive and timely fashion, and to provide suitable options for referral and assistance at home or abroad depending on the situation" (Brunovskis \& Surtees, 2012a, p. 16). Brunovskis and Surtees (2012a) note the main methods through which identification of persons trafficked for sexual exploitation occurs, pointing out that identification mainly happens in the country of exploitation (Brunovskis \& Surtees, 2012a). The authors emphasise that most commonly, it was found that the victims themselves recognised their situation as one of trafficking, 
and thereafter sought out additional actors, that could help them escape the situation they were in (Brunovskis \& Surtees, 2012a). Thus, Richards et al. point to neighbours as one category of actors that victims sometimes approach for help (Richards \& Lyneham, 2014). Another way of identification is by police, often through raids (Amar, 2009; Brunovskis \& Surtees, 2012a). Finally, NGOs, social assistance agencies, as well as persons who are sex workers themselves, may assist in the identification and escape of women from a situation of trafficking (Amar, 2009; Brunovskis \& Surtees, 2012; Richards \& Lyneham, 2014).

\subsubsection{Recovery and (re)integration}

Before turning to the issue of (re) integration in the context of trafficking specifically, a few words should be said about the overall approach to the concept of (re) integration as it pertains to groups close to that of trafficked persons - migrants, forced migrants and refugees. A clear overview of the various definitions of (re) integration that are used in current literature and in practice is given by Koser \& Kuschminder in their study of assisted voluntary return and reintegration of migrants, based on fieldwork done across 15 countries (2015). The two most common definitions used are those offered by the United Nations High Commissioner for Refugees (UNHCR) and the International Organisation for Migration (IOM). According to UNHCR, reintegration is:

"the ability of returning refugees to secure the political, economic, \{legal\} and social conditions needed to maintain life, livelihood and dignity" (UNHCR, 2004 as quoted in Koser \& Kuschminder, 2015, p.14)

Continued:

"Reintegration is a process that should result in the disappearance of differences in legal rights and duties between returnees and their compatriots and the equal access of returnees to services, productive assets and opportunities"(UNHCR, 2004 as quoted in Koser \& Kuschminder, 2015, p.14)

In the context of the same study, the following IOM definition of (re) integration is also offered:

"Re-inclusion or re-incorporation of a person into a group or a process, e.g. of a migrant into society of his country of origin" (IOM, 2004 as quoted in Koser \& Kuschminder, 2015, p.14) 
However, another approach would be to look at (re) integration as only one aspect of the overall process of return, and emphasise the importance of embeddedness as well (Houte, 2014):

"process of an individual's identification with and participation in one or multiple spaces of belonging” (van Houte, 2014, p.27)

Embeddedness is further specified as having three different dimensions: psychosocial, social and economic, and is seen as a process, which is continuous (Houte, 2014).

Nonetheless, as important as the above definitions and approaches to (re) integration are, they fail to capture the specificity of the process for trafficked persons in particular. Thus, when discussing (re) integration of victims of sex trafficking, particular focus must be placed also on the experience of sexual exploitation, and the overcoming of such trauma. In this regard, review of definitions of (re) integration as it pertains specifically to post-trafficking situations, is more adequate. This is the literature that this Chapter turns to now.

\section{Specific to trafficking for the purpose of sexual exploitation}

Once a trafficked person has been identified, the process of (re)integration is initiated. (Re)integration of persons that have been trafficked has been defined as "the process of recovery and socio-economic inclusion following a trafficking experience; it includes settlement in a safe and secure environment, access to a reasonable standard of living, mental and physical well-being, opportunities for personal, social and economic development and access to social and emotional support" (Surtees, 2010, p. 24). Early on in this process, focus is usually placed on dealing with the physical and mental health consequences of the trafficking experience, as well as feelings of fear and shame (Surtees, 2010). These immediate needs are usually addressed by placing identified trafficked persons in a shelter where women are offered counselling services, medical check-ups, and psychological consultation (Bjerkan, 2005; Brunovskis \& Surtees, 2008; Jayagupta, 2009; Surtees, 2010, Vijeyarasa, 2010). Unfortunately, shelter environments have received significant criticism, with the contention being that shelters can have a negative impact on victims. In fact, some researchers have indicated that shelters limit the basic freedoms of the people they seek to help and provide very little privacy (Jayagupta, 2009; Surtees, 2008c; Vijeyarasa, 2010). At the same time, attempting to 'reform' victims and turn them into 'good girls' by teaching them how to dress and act modestly, as has been documented in 
Southeast Europe (Brunovskis \& Surtees, 2008). In this context, prostitution is seen as a form of pathology (Brunovskis \& Surtees, 2008).

Given that most women eventually return to their country of origin, the next steps in the (re)integration process are economic independence and resocialisation (Brunovskis \& Surtees, 2012b; Van Hook et al., 2006; McIntyre, 2014; Pandey, Tewari, \& Bhowmick, 2013). Often, women are offered a variety of vocational training courses and provided with assistance in seeking and obtaining employment (Bjerkan, 2005). Unfortunately, training programs have not always been found to be helpful as some are poorly aligned with current job market demands (Jayagupta, 2009; Pandey et al., 2013) and others have been found to be severely gendered (Kelly, 2003). Additional methods offering potential independence and economic viability include micro credit and social businesses (Van Hook et al., 2006; Surtees, 2012). Recognising the importance of economic independence, calls have been made for greater emphasis on providing financial compensation to victims for the time they spent in a trafficking situation (Kim \& Hreshchyshyn, 2004).

Finally, the recovery and (re)integration of trafficking victims includes a focus on the social environment and, when possible, reunification and reconciliation with family as this is considered crucial to the successful recovery and (re)integration of trafficking victims (Brunovskis \& Surtees, 2012b; Pandey et al., 2013; Vijeyarasa, 2010). In their work focusing on returned trafficked persons from Moldova, Brunovskis and Surtees (2012b) established that conflicts between trafficked persons and their families tend to arise when migration expectations are not realised. They note that ongoing financial difficulties and stigma related to the trafficking experience place additional strain on family relations. In addition, a variety of other factors can inhibit reunification and reconciliation with family. For example, victims may become disappointed when family members fail to meet their expectations with regard to support provision, relationships with children may be hard to re-establish when children harbour resentment and feelings of abandonment, and relationships with partners may be strained by feelings of jealousy (Brunovskis \& Surtees, 2012b).

It should be noted that, to date, no studies exploring the long-term health, economic, and social consequences of sexual exploitation have been conducted. It is, therefore, hard to determine how these health, economic, and social factors interact over time and the degree to which the recovery and 
(re)integration services offered positively or negatively impact the well-being of a trafficked person.

\subsection{Conclusion}

This chapter set out to explore the current academic research and conceptual debates with regard to female sex trafficking, compile the existing information in a comprehensive way, and identify the knowledge that is still lacking. Based on the overall themes discussed in this chapter, several concluding remarks and final recommendations for future research can be made as future research can serve to help policy makers address and tackle human trafficking, while also assisting those that are victim to it.

First, it can be noted that most of the literature available in the field of human trafficking is highly polemic rather than academic. This is due to persistent ongoing debates and disagreement with regard to the definition of human trafficking. Although such literature is necessary and should be further produced to reflect the main discussions from the field, emphasis should also be placed on academic research, which currently is greatly lacking. This challenge could possibly be overcome, by adopting a more interdisciplinary approach to the issue of sex trafficking, and where possible, basing polemic discussions on results gained from research in a variety of fields such as economics, law, psychology, sociology, and medicine.

Second, most of the academic research on human trafficking available is qualitative rather than quantitative. This is likely attributable to the fact that human trafficking is a sensitive topic and an illicit activity. Recruiting the kind of sample sizes necessary for adequate power in quantitative studies is almost always infeasible. Researchers should thus approach efforts to use quantitative methods in this field with considerable reservation.

Third, grey literature, often written in the form of reports, tends to be used quite frequently by policy makers. Unfortunately, in grey literature the methodology applied is often unclear or undisclosed which can lead to misconceptions about human trafficking. We therefore recommend the inclusion of academics in the production of such reports.

Fourth, there have been a multitude of disagreements regarding the interpretation of the concept human trafficking, right from the very conception of the definition of human trafficking up until today. It may be 
time to accept the definition as is, even if some disagreements remain, so as to offer greater flexibility of use when needed in the field. We feel that it is not currently the time to call for changes or revisions to the definition, but rather, we should attempt to better understand the terms that are used within it, starting with 'vulnerability' and 'exploitation'. Academia would be in the best position to make this contribution to the study of human trafficking.

Fifth, when it comes to border controls and migration, current research as well as policy seems to indicate that a choice has to be made, either for or against strict border controls, and for or against freedom of migration. However, research does not reflect such clear-cut solutions. Relatively speaking, for every article that states one position, there is a counterpart that comes to opposing conclusions. Additional research is therefore needed to show the extent to which checks at the border help or hurt the people they claim to protect.

Sixth, traffickers and, in particular, clients are still very much a black box. Debates regarding the definition of a trafficked person have been ongoing. It seems that the definition of a trafficker is also unclear. When does a woman that was initially trafficked, but thereafter chose to remain in sex work and who now charges other women in sex work for protection cease to be a victim and become a perpetrator? There may be some information and research about clients of sex workers, but very little is known about those persons that purchase the services of trafficked individuals. Regarding both traffickers and clients, there are certain sources of information and methodologies of research that have not been fully utilised, such as criminal investigation reports in possession of the police and social network analyses.

There is significant literature on the health consequences of human trafficking. However, we must ask how health, social, and economic factors linked to recovery and (re) integration interact, particularly in the long run. Financial need seems to be one of the primary drivers of vulnerability to trafficking but we need to know more about the role economic necessity plays in decision making among trafficked persons after their trafficking experience, as well as the importance of financial compensation for their recovery.

Finally, female sex trafficking appears to be a polarised field in which policy makers as well as researchers feel compelled to uphold one extreme position or another. However, based on the literature reviewed, we contend that what 
is most necessary is research that, regardless of direction of the conclusions, is based on sound scientific evidence above all else. 


\section{Chapter 3: Methodology}

\subsection{Introduction}

The research field of human trafficking is riddled with an array of challenges, not just in terms of knowledge gaps and open questions that still need answering, but also, in terms of the methodologies available for gathering data. One of the primary reasons for this is the fact that the main actors - the persons trafficked, as well as the traffickers, belong to the group of 'hidden populations', "a group of individuals for whom the size and boundaries are unknown and for who no sampling frame exists" (Tyldum \& Brunovskis, 2005, p.18). Belonging to such populations thus "involves stigmatised or illegal behaviour, leading individuals to refuse to cooperate, or give unreliable answers to protect their privacy" (Heckathorn, 1997 as quoted in Tyldum \& Brunovskis, 2005, p.18).

From the very beginning, this thesis has been an attempt to identify and then address one of the existing gaps in human trafficking literature, and to, in the process, use the most adequate methodologies available for studying the aforementioned 'hidden populations'. What this chapter will do is: (1) detail the decision making process that led to the research question, (2) discuss in detail the methodologies used to gather data and the way they were adapted throughout the fieldwork phase due to challenges on the ground and finally, (3) discuss the analysis and writing process. The chapter will conclude with a focus on some of the shortfalls of the research, and recommendations for the future, with regard to human trafficking research and methodologies used.

\subsection{Formulating the research question}

At the onset, there were three distinct cycles of literature review undertaken, throughout the first two years of research. The first was to determine relevant areas of study within human trafficking in general, that are both academically necessary and practically applicable. Once the focus on recovery and (re) integration was decided, a second literature review was undertaken to study the recovery and (re) integration process, not only in the area of human trafficking, but also of related groups of persons such as migrants, refugees and forced migrants, ex-combatants and child soldiers, persons raped and 
victims of domestic violence. The purpose of the second cycle of literature review was to determine the best theoretical framework through which to approach the issue of recovery and (re) integration in the context of human trafficking. The first and second cycle of literature review conducted, entailed the study of in total 438 papers, books and reports. ${ }^{7}$ The databases used were Google Scholar, PsychINFO, Web of Science, ProQuest, Scopus and Medline. The keywords (and also areas of study) used in the searches to compile the documents were: migrants (return and (re) integration), forced migrants (return and (re) integration), ex combatants, child soldiers ((re) integration), rape (recovery), violence against women (recovery), human trafficking (numbers and definitions), human trafficking (security), human trafficking (migration), human trafficking (human rights), human trafficking (smuggling), human trafficking (prostitution), human trafficking (causes), human trafficking (recruitment), human trafficking ((re) integration), and finally, within (re) integration as it pertains to human trafficking, the subtopics: definitions, health, social, economic, long run.

The third and final literature review was undertaken to delve deeper and integrate all of the above areas of study, but now with a clear formulation of the overall research question of the thesis, as well as the theoretical framework to be used, in mind. The results of the final literature review are presented in Chapter 2: Female Sex Trafficking Conceptual Issues, Current Debates, and Future Directions, of this thesis.

The following section will describe in greater detail each cycle of literature review undertaken throughout the first two years of research.

The research process began with a thorough review of literature on the topic of human trafficking, with the purpose of identifying knowledge gaps, with practical relevance, in said literature. One of the principle topics of interest that arose was recovery and (re) integration of persons trafficked for the purpose of sexual exploitation. The emphasis on recovery and (re) integration is not solely due to its academic value, but also to its practical application and usefulness for those most hurt by the trafficking process - the victims. It is policy relevant research that can be used in the future to determine what could be useful measures to be undertaken in addressing the needs of persons that have been trafficked. One of the most severe forms of trafficking identified was for the purpose of sexual exploitation. Thus, women, who were found to

\footnotetext{
${ }^{7}$ The resulting document of the second literature review, which discusses the relevant points of the material that was studied is available upon demand.
} 
be the predominant victims of trafficking for the purpose of sexual exploitation, became the target group of this research (ILO, 2012).

Once the relevance and overall importance and applicability of recovery and (re) integration research of women trafficked for the purpose of sexual exploitation was determined, the literature was reviewed to identify what aspects of the recovery and (re) integration process to focus on, based on the existing identified gaps. It was determined that although we have some knowledge in regard to the recovery and (re) integration process, there is little information about the long-term outcomes and results of the same.

Thus, the principle research question was formulated:

\section{What determines successful recovery and (re) integration of women trafficked for the purpose of sexual exploitation, and how do these factors interplay, in the short and long run?}

A second review of literature was undertaken, with a particular focus on recovery and (re) integration processes, now not only focusing on human trafficking victims, but also on related groups such as migrants, refugees and forced migrants, ex-combatants and child soldiers, persons raped and victims of domestic violence..$^{8}$ It was found that the literature on the recovery process

\footnotetext{
${ }^{8}$ For literature on reintegration of migrants refer to: Cassarino, 2010; Constant \& Massey, 2002; Dustmann, 1997; Dustmann, 2003; Dustmann \& Weiss, 2007; King, 2002; Vlase, 2013; Zhang 2013; Diatta \& Mbow, 1999; Ilahi, 1999; Merkle \& Zimmermann, 1992; McCormick \& Wahba, 2003; Rooth \& Saarela, 2007; for literature on reintegration of refugees and forced migrants refer to: Chimni, 2002; Özerdem \& Sofizada, 2006; AbbasiShavazi \& Glazebrook, 2009; Farwell, 2001; Haider, 2009; Zimmermann, 2012; Bascom, 2005; Kibreab, 2002; Cahn, 2006; Gillespie Karl, Peltzer, Malcolm Ma, 2000; Steflja, 2012; Black, 2002; Al-Ali, Black, \& Koser, 2001; Black, Koser, Munk, Atfield, D’Onfrio \& Tiemoko, 2004; Arb, 2001; Koser, 2001; for literature on reintegration of ex-combatants and child soldiers refer to: Watteville, 2001; Annan et al. 2011; Dzinesa, 2007; Veale 2011; Muggah 2007; Brooks 2012; Derluyn et al., 2004; Kohrt, Jordans, 2008; MacMullin and Loughry, 2004; Shepler 2005; Betancourt et. al., 2010; Boothby et al., 2006; Leff, 2008; Stark et al., 2009; Stovel, 2008; Williamson, 2006; Betancourt et al. January 2010; Humphreys and Weinstein, 2007; Knight and Ozerdem, 2004; Paes, 2005; Peters, 2007; Zack-Williams, 2006; Boas and Hatloy, 2008; Wessels, 2004; Specht and Attree, 2006; for additional literature on reintegration of persons raped refer to: Bownes et al., 1991; Burgess and Homstrom, 1978; Burt and Katz, 1988; Frazier, 2000; Frazier and Burnett, 1994; Frazier and Schauben, 1994; Hill and Zautra, 1989; Kelland, 2012; Littleton and Breitkopf, 2006; Regehr et al., 1999; Resick, 1993; Roth et al. 1990; Rothbaum et al. 1992; Ullman, 1997; Braithwaite, 2006; Campbell and Ahrens, 1998; Kinyeki, 2013; Ptacek, 2009; Ullman,
} 
of rape victims was most relevant for designing the theoretical framework through which to approach the issue of recovery and (re) integration of women trafficked for the purpose of sexual exploitation. Of particular interest, was the literature that looked at sexual assault recovery through the application of the ecological model of human development (Neville \& Heppner, 1999; Campbell, Dworkin \& Cabral, 2009; Harvey, 1996; Harvey, 2007). Neville and Heppner (1999) review literature on recovery of rape victims through the development of CIEMSAR (the Culturally Inclusive Ecological Model of Sexual Assault Recovery). They look at factors at the micro (individual), meso, and macro levels, which influence the recovery of a person after a sexual assault. At the micro level, they look at the characteristics of the rape, the person specific variables, cultural factors and coping behaviours. At the meso level, the focus is on social support from friends and family, as well as institutional intervention. Finally, at the macro level, the broad sociocultural context is looked at, as well as racial and ethnic factors. Campbell, Dworkin \& Cabral (2009) have a similar approach in their review of literature on rape recovery, except they add another level of analysis, factors related to the 'chronosystem', which "encompasses the changes that occur over time between persons and their multiple environments" (Campbell, Dworkin \& Cabral, 2009, p.227). Harvey (1996) looks at the recovery of victims of trauma, and again uses the ecological model in approaching the issue. What is particularly of interest is the understanding of recovery as a 'multidimensional phenomenon', whose success or failure can be judged on the basis of several outcome criteria, taking into consideration the context of each individual case. Thus, Harvey (1996) concludes, adequate interventions cannot be a one size fit for all, but must depend on what is the best 'ecological fit' for the particular individual case, again, depending on the particulars of the context and circumstance.

Based on the literature dealing with rape recovery using the ecological model, the following sub-questions of this thesis were formulated:

1999; Bletzer and Koss, 2006; Burgess and Holmstrom, 1979; Cohen and Roth, 1987; Ellis, 1983; Kilpatrick, 1981; Burt and Katz, 1987; Thompson, 2000; for literature on reintegration of female victims of violence refer to: Bennett and O'Brien, 2007; Fischbach and Herbert, 1997; Koss et al., 2003; Helfrich and Aviles, 2001; Hovell, 2006; Curtis-Fawley and Daly, 2005; Herman, 2003; Cribb, 1999; Few, 2005; Surtees, 2003) 
(1) What is to be considered a success or failure of the (re) integration process?

(2) What are the influencing factors on the (re) integration process in the short run, and in the long run?

(3) How do these factors interplay, and which may be deemed as more and which as less important for the overall (re) integration of the trafficked person?

Once the main question and sub-questions of the thesis were formulated, and the target group was selected, it was necessary to conduct one final review of the literature, in order to collect all research on the now identified topics of study, but also define the main concepts that would come up throughout the research. The results of this third and final literature review are presented in Chapter 2: Female Sex Trafficking Conceptual Issues, Current Debates, and Future Directions.

\subsection{Research design: location, target groups for data gathering, research strategy and questionnaire development}

\subsubsection{Location}

Origin countries of Southeastern Europe and destination countries of Western Europe, mainly and primarily the Netherlands and to a lesser extent Italy, were chosen as the geographical focus of this study. The reasons are manifold. It has already been pointed out that trafficking for the purpose of sexual exploitation was chosen as a focus, as one of the most sever forms of human trafficking. Women were chosen as the target group of study as they present $55 \%$ of the victims of trafficking for the purpose of sexual exploitation (ILO, 2012). Having in mind the identified target group and type of trafficking to be studied, the researcher looked at the geographical prevalence of trafficking for the purpose of sexual exploitation per 1000 inhabitants. It was found that the highest prevalence for such trafficking, of 4.2 per 1000 inhabitants was in Central and Southeastern European countries, as countries of origin (ILO, 2012). Looking more closely to the region, having in mind also the origin of the researcher, the following countries of focus were chosen: Albania, Bulgaria, Bosnia Herzegovina, Croatia, Macedonia, Montenegro, Serbia and Kosovo. It should be noted that the researcher comes from Macedonia and has previously worked extensively throughout the region. It was expected that 
in terms of understanding the context as well as language and logistical factors, the choice of the above noted countries for study was best.

However, when studying recovery and (re) integration it is not sufficient to only look at countries of origin, for the simple reason that some victims stay in the country of destination and the recovery process begins there. Thus, to have a more complete idea of the dynamics of the process, destination countries had to be included in the study. It is found that the majority of victims of trafficking for the purpose of sexual exploitation from Central and Southeastern Europe are trafficked to Western and Southern Europe (UNODC, 2012). Having this in mind, two countries were chosen, the Netherlands and Italy, as destination countries to be included in the study, as it was found through the initial fieldwork that many victims from countries such as Albania and Bulgaria, were trafficked to Italy and the Netherlands. Due to time pressures only one interview was conducted in Italy.

\subsubsection{Target groups for data gathering}

Having decided the geographical focus of the study, it was then important to determine the target groups from whom data would be gathered. The primary target group was that on whom the research is based, namely women that have been trafficked for the purpose of sexual exploitation. At this point, a few words should be said regarding the terminology used when referring to this group. The researcher is fully aware of the discussion amongst academics and practitioners, to avoid the use of the term 'victim' and use the term 'survivor' in its place. However, for the purpose of this study, these terms will be used interchangeably, as well as most often, the term 'trafficked person' to refer to anybody belonging to the main target group of the study. The reason for this is the fact that not all women may prefer to be called 'survivor', and even further, this term may not be a good descriptor of their current situation. It may be that for some women, using the term 'victim' is a recognition of the suffering they have endured. Finally, in certain cases, and countries, a person must be identified as a 'victim' in order to be recognised as a person trafficked. Although it should be said that the term most commonly used by organisations and service providers working with trafficked persons is 'beneficiaries'. Nonetheless, this study will use the term 'victim', 'survivor', 'trafficked person' and 'beneficiary', in order to refer to those belonging to the main target group. The interchangeability of these terms in this context should be taken to mean that this group of individuals should not be solely labelled as one or the other, and what term is used should ultimately be up to 
the person to whom it refers, and dependent on their context and circumstances.

The primary group from which information was gathered for this thesis was service providers. Service providers were defined as any person who has had direct contact with a victim of trafficking. Namely, police, lawyers, doctors, social workers, case workers, psychologists, psychiatrists, teachers, hot-line operators and other types of workers at non-government as well as government organisations. These groups have direct contact with victims of trafficking, and as such are a source of knowledge regarding the recovery and (re) integration process of trafficked persons. Some service providers such as case workers, psychologists and psychiatrists, and social workers may have had continuous contact with victims lasting for many years. In this regard, once again, they are a precious source of knowledge. In addition, gathering information from them would avoid the danger of re-victimising the trafficked person.

It was also decided to gather information from trafficked persons themselves, however, for them not to be the primary source, due to serious ethical reservations. It may be argued that questioning about the trafficking experience, and any attempt at recollection may be cause for re-victimisation of the trafficked person. That is why measures were undertaken, whenever a trafficking victim was interviewed, to ensure that secondary victimisation will not happen, as far as that is possible. The measures will be discussed in greater detail later on.

Finally, it was also decided to aim for gathering information from family members or friends of trafficked persons as well, only when possible. However, having in mind that in many cases, friends and family may not be aware of the trafficking experience, there is always the danger that attempts to speak to them would expose the victim. Thus, at the end, only one such occasion occurred in the context of this study.

\subsubsection{Research strategy and questionnaire development}

There were many different options considered regarding the strategy to be used in gathering data for this study. As noted, data is gathered from trafficked persons, service providers and potentially, where possible, friends and family of trafficked persons. 
Since information needed to be gathered in regard to particular topics related to the recovery and (re) integration process of trafficking victims, it was decided to use semi-structured questionnaires. It was decided that victims would also be asked about the trafficking experience, however, if possible, the researcher would get the green light from the service provider familiar with the case, to ensure that no re-traumatisation happens. In cases where victims agreed to discuss the trafficking experience but would start showing signs of distress, it was decided to stop the line of questioning, and move on to other parts of the interviews.

Having in mind that trafficking victims belong to the group of 'hidden populations', mentioned at the beginning of this chapter, it was necessary to ensure that the information gathered from them was indeed reliable. Thus, the information gathered from victims was to be triangulated, through consultations with service providers familiar with each trafficking case prior to and, where possible, post interview of the victim.

Since specific topical data was to be gathered from the service providers as well, the method used was to be semi-structured questionnaires.

When it comes to participant observation in the context of human trafficking, some researchers have raised reasons for caution. According to Brunovskis \& Surtees (2010), there are ethical considerations when one considers the relationships which may be built with victims through participant observation and continuous contact with the victim over time, creating expectations from the researcher. Having this in mind, as well as the time constrains of the research, it was decided to meet only once with each respondent. However, no time limitations were placed on the length of the interviews. In addition, one victim was interviewed in the presence of her entire family (sister, mother, and brother) at her home. Thus, this gave at least one opportunity for familiarisation, creating a closeness and as a result, participant observation in an intimate setting was possible.

Acknowledging that there are two groups of interviewees, the victims and service providers, two different questionnaires were developed. The questionnaires were developed using the theoretical framework presented above as a basis.

At the micro level, information was to be gathered on the personal characteristics of victims, role of religion, education, agency, perceptions of 
stigma, coping, pre-trafficking related experiences, and trafficking related experiences. At the meso level, information was to be gathered on participation in (re) integration programmes, relations with family and friends, and community attitudes. Although the scope of the study did not allow for a thorough investigation of macro level influences, the researcher still investigated the socio-cultural context of the region/countries under study, attitudes toward sex work, as well as the economic and political situations.

Based on the above presented thematic areas of study at the micro and meso level, and to a certain degree the macro level, the questionnaires were designed. The full questionnaires can be found in Annex 2 .

Different interview protocols and questionnaires were used for trafficking victims and service providers. The questionnaire for trafficked persons covered several general areas, focusing on 1) life before the trafficking experience, 2) the trafficking experience itself; how it happened and what helped the person to get through it, 3) how the identification as 'trafficked' occurred, 4) description of the period immediately following trafficking, including questions having to do with experience of stigma, and participation in assistance programs, 5) description of economic situation at the time of the interview, 6) description of social life, mental and physical health at the time of interview, and finally, 7) description of how the person envisions the future. As the focus of this research is the recovery and (re) integration process not just immediately following the trafficking experience, but also in the long run, there was a particular focus on the current situation of the victim, in particular if several years had passed since the trafficking experience.

A different interview protocol was used for service providers, covering a variety of questions. Service providers were asked about their daily work and tasks; about how they come in contact with trafficked persons (how victims are identified); what is their initial approach when they come in contact with trafficked women for the first time (what are the needs of the women, what do they want, what do they tell the service provider, how does the service provider interact with them); what the challenges are, during these initial contacts; how interactions proceed as time goes by (how do the needs and wishes of the trafficked women change with time; how long do service providers follow up with each case, what are some of the challenges in later interactions) and what according to the service provider constitutes successful (re) integration (with examples given of successes and failures of the (re) 
integration process). Since one of the primary goals of this study was to look at the recovery and (re) integration process in the long run, particular attention was given to experience service providers had with victims several years after exiting the trafficking situation. They were asked to give examples of some of the interactions with beneficiaries who either stay in regular contact or contact them years after the initial granting of services. Service providers were also asked about organisational, societal, as well as personal attitudes toward sex work.

\subsection{Implementation: sampling and outreach, pilot study, interviews and other data gathering strategies}

\subsubsection{Sampling and outreach}

Fieldwork began with the compilation of a list of organisations, nongovernmental as well as government institutions, national and international, that deal with issues of human trafficking in Southeast Europe, the Netherlands and later on in the research, Italy. Organisations and institutions were then contacted via email and introduced to the research. The contacts were asked if they could put the researcher in touch with employees that work directly with victims of trafficking, and also, if the researcher can conduct interviews with their beneficiaries, those that are the victims of trafficking. It was only certain organisations and shelters in Albania, Bulgaria, Bosnia Herzegovina, and the Netherlands that were open to allowing access to their beneficiaries. One non-governmental organisation in Bosnia Herzegovina on the other hand was unwilling to put the researcher in touch even with service providers that are in direct contact with victims, let alone the victims themselves. The assumed reason for this was that service providers may unintentionally reveal personal information regarding their beneficiaries. All organisations contacted in Serbia were not willing under any circumstances to allow interviews of their beneficiaries. The reason given was negative experiences in the past, where victims were re-traumatised, or in the case of some organisations in Albania, publication of confidential personal information of victims. Some countries such as Macedonia were either unresponsive to attempts by the researcher to arrange for any kind of interviews or replied by referring the researcher to studies published about the country by organisations such as the IOM. Thus, no respondents are included from Macedonia. Additionally, although attempts were made to gain access points to anti-trafficking organisations in Croatia and Kosovo, none were successful. 
Through initial contacts established, and referrals received by the researcher, it became evident that most organisations in Southeastern Europe that work in anti-trafficking are familiar with each other, across countries. Even further, it was found that there are regular exchanges of know-how between origin and destination countries, such as between anti-trafficking organisations in Bulgaria and Serbia on the one hand, and the Netherlands on the other.

Nonetheless, it should be noted that the sampling and selection process of respondents for this study was made on the basis of research into the dominant actors in the field of anti-trafficking in each country, and referrals from various contacts of the researcher. In this regard, they are not allencompassing and representative of all existing organisations that work in this field. However, it should be noted that the main and/or largest antitrafficking organisations working in Albania, Bosnia Herzegovina, Bulgaria, and Serbia, as well as the Netherlands, have been included. In addition, organisations, as well as victims interviewed, do not originate only from capitals or major cities, but also from smaller towns and villages. A complete list of organisations can be found in Annex 3.

All trafficked persons interviewed for this study were approached through an official institution or organisation that offered some kind of service, was responsible for their identification as trafficked, or received them upon return to the country of origin. Methodology related literature from the field of human trafficking identifies such institutions/organisations as 'gatekeepers', guarding the victims from intruding researchers (Brunovskis \& Surtees, 2010). There are certain risks with this approach in that the researcher gets access only to those victims that have been identified as such, and which have been chosen by the 'gatekeeper' not by the researcher. Nonetheless, the organisations that granted access to victims in Bulgaria and the Netherlands seemed to have approached indiscriminately all the beneficiaries that were currently under their care, not only those deemed as 'good examples'. All service providers interviewed were also open about negative examples and failures of their processes of recovery and (re) integration. The shelter visited in the Netherlands introduced the researcher to all the victims originating from Central or Southeastern Europe currently residing there and let each beneficiary decide for herself if she would like to be interviewed. In addition, the IOM, which facilitated the access to beneficiaries in Bulgaria, attempted to call every beneficiary from their database that fulfilled the criteria of the research (victim of sex trafficking), in the presence of the researcher, in order to ask if they would be willing to participate in the research. Only those that 
were still in danger from the trafficker, which would then put the researcher in danger should she speak to them, were left out. Nonetheless, the overall response rate, as evident by the number of victims interviewed, was low. In some cases, it was due to the fact that the victim had moved on with her life and no longer wanted to be reminded of her past experiences. In other cases, the organisation simply did not have up to date contact information, perhaps in a deliberate attempt by the victim to cut all ties with the past life. It was also evident that some victims interviewed in Albania and Bosnia Herzegovina, had agreed to the interview due to their gratitude toward the organisation that introduced the researcher.

Finally, it should thus be said that the victims interviewed for this study are in no way representative of the entire group of women trafficked for the purpose of sexual exploitation. It is a limited group of individuals explicitly identified as victims, and all but one victim had received some kind of assistance from anti-trafficking organisations. Nonetheless, their input was extremely valuable and informative. The researcher decided against the socalled 'chain referral' strategy, which is asking a victim to refer another victim (Brunovskis \& Surtees, 2010). Victims were either reluctant to speak about any other women they knew, or it was obvious that they did not have contact with other women in a similar situation. In many situations, the researcher felt that this would be an inappropriate question to ask. This is consistent with other human trafficking methodology literature that finds trafficking victims to be 'non-associative', 'hard to reach' populations that do not have or form networks (Brunovskis \& Surtees, 2010, p.23). The complete list of respondents with a short profile of each can be found in Annex 4.

\subsubsection{Pilot study}

The interview protocol was initially tested through a pilot study that was conducted in Albania between the 25 and 29 November 2013. The pilot consisted of visiting one residential shelter, in the east of Albania, managed by a local non-government organisation. The researcher interviewed shelter coordinators, a psychologist, and two victims of trafficking. In addition, the researcher was granted access to two beneficiaries who were introduced as 'at risk of being trafficked'. It was explained that the two beneficiaries who were sisters, were brought to the shelter as young children, after the loss of their mother as their father could not care for them. As the public focus on the issue of human trafficking grew over the years, the shelter determined that the sisters would get more financial benefits, at a time when grants for anti- 
trafficking efforts were available, if they were labeled as 'at risk' for trafficking.

Finally, one of the trafficking victims was interviewed in her home, in a small village in the south of Albania. The interview was conducted in the presence of her mother, brother, and sister. The sister was very talkative and also participated extensively in the conversation with the researcher. This was also a case that gave the opportunity for participant observation, as the researcher could see the family dynamics, and could observe the trafficked person in her safe environment. The role her family played, especially the dominant role of her sister, was one of the crucial factors for her successful recovery and (re) integration from the trafficking experience.

Throughout the pilot study, as the researcher did not speak Albanian, a professional translator was used. For one of the interviews, the case worker of the victim was used as a translator. Professional translators or service providers were used interchangeably throughout the rest of the study, whenever translation was necessary.

The use of a translator, as well as the use of a person familiar with the victim but who is not a professional translator, both have their positive and negative aspects. Using a translator was positive in that the translator was told to be as precise and objective as possible, and to not intervene or interject in the interview process. On the other hand, the use of the case worker who was intimately familiar with the victim was of great value, as certain timelines and answers were clarified, or given vis-à-vis the professional opinion of the service provider. Inconsistencies were easy to spot and were then explained. The case worker also immediately noticed the discomfort of the victim when going into the particulars of the trafficking experience, so the interview moved on to other questions and thus avoided the re-traumatisation of the victim.

It was evident from the pilot interviews that there would inevitably be moments when the victim may not be comfortable speaking about the negative experiences of the past, and such instances of distress were avoided by turning to some more positive aspects of the present situation (for instance, if the victim had mentioned she has children and how much she loves them, the researcher would briefly divert to this topic, before continuing with the rest of the questions). In addition, it also became evident that there would be respondents, in particular victims, who would have language difficulties 
(even with their mother tongue) or would simply fail to understand certain questions, no matter how clearly they were stated. The use of prompts or very general, open questions such as 'what motivates you?' or 'how do you feel now?' and 'what makes you happy' were found to be the most efficient in eliciting a response from the victim in these situations.

At the end of the pilot study, it was found that no major changes needed to be made to the interview protocol. Thus, it was decided that the interviews of the pilot study would also become part of the main study and be analysed as such.

\subsubsection{Interviews and other data gathering strategies}

The main research was conducted in Albania, Bosnia Herzegovina, Bulgaria, Montenegro, Serbia, the Netherlands, and Italy. The first interviews were conducted 4 November 2013, and the last on 30 December 2015. A total of 52 semi-structured interviews were conducted. Interviews lasted for 20 minutes to over 2 hours in length. Interviews with service providers were mostly conducted in the offices of assistance organisations, while for victims these took place in the locations where they felt most comfortable, such as the offices of assistance organisations, cafes, or in one case, the home of the victim where the family of the victim (mother, brother ,and sister) could also be present and participate in the conversation. A total of 9 victims of trafficking, 8 female and 1 male were interviewed, all victims of trafficking for purpose of sexual exploitation. 4 of the victims ( 3 female and 1 male) were from Bulgaria and trafficked to the Netherlands, Spain, and an undisclosed country of destination, 2 of the victims were from Albania and trafficked to Italy and Greece, 2 were from Romania and trafficked to the Netherlands, and 1 from Bosnia Herzegovina was trafficked internally. Ages ranged from 22 to 42 , while the duration of the trafficking experience ranged from 14 days to 3 years. Although it was not always possible to ask or to understand how much time had passed since the trafficking experience, for those victims that made it clear, time since the trafficking experience ranged from several months to 10 years. In addition, 2 women were sisters, who as young children were identified as 'at risk' of being trafficked, and as such were placed in a shelter, where they reached adulthood. Finally, one of the victims from Albania, as already noted, was interviewed at her home, with the presence of her mother, brother and sister. The sister of the victim was also participating in the conversation, and was included in the interview.

Service providers were from a range of professions, where the educational background did not always coincide with the current assistance role in the 
recovery and (re)integration process. Overall, 40 service providers were interviewed, in Albania (11), Bosnia Herzegovina (5), Bulgaria (5), Italy (1), Montenegro (2), the Netherlands (6) and Serbia (10). The educational background or organisational role of those interviewed was that of case manager (2), children center coordinator (1), clinical social worker (1), crisis unit operator (1), day center coordinator (1), NGO or government agency director (4), member of female NGO lobby (1), hot line operator (1), government worker (1), lawyer (2), police official (2), program manager (3), project coordinator (1), psychologist (7), psychotherapist (1), shelter coordinator (2), shelter staff (3), social worker (2) and course teacher (1).

The guidelines of the World Health Organisation were followed in conducting interviews with victims of trafficking and service providers. In addition, it should be noted that the questionnaires of this study were reviewed and approved by the Ethics Committee of the Faculty of Psychology of Maastricht University.

Nonetheless, some observations and notes on the interview processes are warranted. Firstly, it should be noted that one interview with a male victim of trafficking for the purpose of sexual exploitation was conducted and is considered as part of this research. The interview was not planned in advance, and was not sought out specifically. It occurred in Bulgaria, where the 'gatekeeper' organisation informed the researcher of the possibility of interviewing a male victim. Although such an interview would not be representative of male victims in any way, nor have any necessary direct relation with female victims, the researcher saw this as a unique opportunity for gaining some insight into a different category of victims, and perhaps provide some limited contrast to female victims of trafficking for the purpose of sexual exploitation. Having said that, it should be emphasised that at all occasions through this thesis where data from this particular interview is presented and analysed, it is explicitly noted that it comes from the single interview of a male victim of trafficking.

The greatest challenge in interviews with the trafficking victims had to do with the establishment of trust between interviewer and interviewee. Building trust is key to receiving reliable information from victims in these contexts. However, it should be kept in mind that the researcher met with each victim only once, and only for up to several hours, and thus establishing trust was a challenge. It was found that the most efficient way of overcoming this was through the help of the 'gatekeepers'. Thus, in situations where the victim had 
trust in the service providers, it translated into trust in the researcher. However, in one case the victim conveyed her disappointment with the shelter at which she was staying, to the researcher. No shelter staff was present at that interview. Another method of building trust was to give some personal information by the researcher, or to speak about hobbies such as cooking. These strategies were also useful in relaxing the interviewee.

An additional challenge in the interviews with victims was in situations where the victim had a hard time expressing herself, in some cases due to mental problems. In these cases, it was found that the assistance of a service provider familiar with the victim throughout the interview process was of significant help.

Wherever possible, service providers were consulted about the particular cases of the victims to be interviewed ahead of the interview with the victim. In some cases, there were discussions with service providers about particular victims following the interview. Doing so, allowed for inconsistencies or misrepresentations in the narrative of the victims interviewed to be uncovered. For instance, one victim throughout the interview kept referring to the man that asked her to become a sex worker as her 'husband'. It was subsequently noted by her case worker that not only was the man she mentioned the trafficker, but that they were never married. Even further, the fact that she lived with him, and had a child with him out of marriage, was the primary reason her father would not speak to her. Thus, her representation of the man to the researcher, as her 'husband' exposed the stigmatisation she still feels as a consequence of this situation and the general weight put on marriage by the community to which she belongs.

The benefits of having a service provider present at some of the interviews with victims of trafficking have been noted. However, it should be pointed out that when victims in these contexts were asked about their satisfaction with the service provided by the host organisation, they always responded in a positive way. The objectivity of these answers should be questioned in these situations.

Furthermore, although having interviews at the offices of host organisations provided safety for both victim and researcher, the interviews at cafes or, in one case, at the home of the victim were much more relaxed and did not have the official air, and in some cases urgency, of the interviews conducted in more formal locations. Still, it should be acknowledged that having interviews 
in cafes, particularly in the small towns of origin of the victims, did expose both victim and researcher to potential danger. In one instance, the researcher travelled to a small town in Bulgaria, where the victim was living and the anti-trafficking organisation did not have a presence. It was disclosed at the interview in a local cafe that the victim had been out of the trafficking experience for only a few months, and that the trafficker has not been captured, has just re-entered Bulgaria, and in the words of the victim, "may appear at any moment". Although no dangerous situation occurred, the risk in this occasion was significant.

Finally, a few words about the interviews conducted with service providers. Those with many years of experience were a wealth of knowledge and full of practical examples. The service providers that were relatively younger in regard to experience, were hopeful and positive about their beneficiaries. Overall as a group, many were reluctant to accept an interview, or accepted to do the interview but with little enthusiasm, seemingly due to work overload (in many occasions this was evident once the researcher arrived at their offices). There were 2 separate occasions where the service providers had forgotten that interviews were set up, until the moment the researcher arrived for the interview. However, once the interview began, respondents spoke extensively and with energy and passion about their work and the people they served. Also, they were open about successes and failures they have experienced in their job.

Overall, for interviews conducted in Southeastern Europe, the fact that the researcher originates from Macedonia was of great use, as she was familiar with the cultural context and was not seen as an outsider by the service providers and, in some cases, by the victims as well. Where possible and adequate, the researcher also shared personal information and personal background, specifically, her new role as mother, in order to relate to the respondents - in particular respondents (service providers and survivors) who were parents themselves. For instance, during one interview session, the respondent who was a survivor attended the meeting with her daughter who was several months old. As the baby was crawling around, the researcher and respondent exchanged experiences of feeding and basic care of small children. In the process, the respondent also disclosed some hygiene challenges of women with small children at the shelter. 


\subsection{Analysis and writing}

The interviews conducted were in English, Italian, Bosnian, Bulgarian, Montenegrian, Serbian, and Albanian. The English language interviews were transcribed verbatim. The interviews that were conducted in Bosnian, Bulgarian, Montenegrian, and Serbian were translated and transcribed from the language of the interview directly into English by the researcher. As the researcher does not speak Albanian and Italian, Albanian and Italian segments of interviews were translated and transcribed into English by a native speaker. The segments that were translated into English by a translator or service provider during the interview were transcribed by the researcher.

During the fieldwork phase, the researcher followed a precise procedure during and after each conducted interview. Firstly, each respondent was asked if the researcher could record the interview, and permission was granted in all 52 interviews. Following each interview, the researcher reviewed the handwritten notes, and made additions if necessary. The researcher also kept a 'research diary'. In the research diary notes and adaptations, if needed, were made in regard to the methodology, theoretical framework, and general tips for each subsequent interview. In cases where particular themes of interest had come up, note was made of this, and additional questions were added to future interviews, in regard to that topic. For instance, in some cases it was found that much more informative answers were given by service providers when they were asked to contrast sex work to human trafficking, instead of being asked about their attitudes toward sex work. This occurrence was noticed, and thereafter incorporated in the interview protocol where possible.

Following the fieldwork phase, transcriptions of all 52 interviews were entered into QSR Nvivo 11.2.2 (1707) for Mac, which would be the software programme used for the analysis of the data. Once all interviews were entered in Nvivo in a uniform format, the analysis of the data began. The analysis consisted of several phases:

1. All data was read and re-read several times. In addition, 'memos' were attached to each interview of a victim of trafficking that contained observations by the researcher and some contemplations in regard to each case.

2. Open coding was then used to divide every interview into segments by a more general theme that emerged, often coinciding with the distinct topics 
covered by the questionnaire (Flick, 2009). The resulting codes were the main thematic codes that emerged.

3. Each theme resulting from the open coding was then read and in vivo and descriptive sub-codes were used to identify relevant and explanatory concepts within each theme.

4. Next, axial coding was used to map relations between identified sub-codes within each categorical code, as well as relations between sub-codes and the main code to which they were assigned.

5. Finally, mind maps were used to relate the main codes to one another, as well as map any relations between sub-codes. The conclusions induced were written up and contrasted against the hypotheses that were initially derived from the theoretical framework, as well as other existing theory.

\subsection{Challenges, limitations, recommendations}

Some of the main challenges encountered throughout this study were already mentioned. The primary difficulty remains gaining access to victims of trafficking. It was noted that anti-trafficking organisations often serve as 'gatekeepers', and rarely give researchers the possibility to speak to their beneficiaries. Several organisations have the explicit policy of not granting interviews with trafficking victims, while one organisation also had a policy to not allow any service provider that has direct contact with victims to speak to the researcher. In cases where the organisation did grant access to victims, it was often not possible to reach the beneficiaries, in particular those that had been trafficked several years in the past, as they had changed their contact information or were not answering phone calls placed from the organisation. These are the primary reasons why the number of interviews with victims of trafficking is low.

Again, as already noted, interviews were conducted in Albanian, Bulgarian, Serbian/Bosnian/Montenegrian, English and Italian. A translator was used for interviews conducted in Albanian and Italian, while the researcher conducted all interviews in Bulgarian, Serbian/Bosnian/Montenegrian and English. In certain cases, a professional translator was used, while in others a service provider adopted the role of translator as well. It should be noted that in some cases the presence of service providers at some of the interviews with victims, may have had a positive influence on the victim, as they are most often individuals which have gained the trust of the trafficked person. However, the answers of the victims in these cases may have been impacted by this presence in a negative way as well, in particular in regard to the truthfulness of answers 
in regard to services provided by the host organisation to which the service provider present during the interview belonged to. Questions were also in some cases shared with the service providers familiar with the victims ahead of the interview, to ensure that there are no inquiries which may disturb the victim. This however gives great influential power to the service provider as to what topics may be discussed with the victim. In certain situations, however, this could not be avoided as service providers explicitly asked for the interview protocols.

As already acknowledged, some of the victims interviewed showed visible difficulties of verbal expression, while others reluctance to speak openly or extensively to the researcher. In certain cases there were discrepancies between the information given by the victim during the interview and the information given by the service provider in regard to that victim. In some cases, the questions were re-formulated in a way that was more easily understandable for the victim. Finally, on a few occasions, when the interviewee became visibly disturbed by some of the topics of discussion, the conversation was continued in a different direction, thus, leaving some questions from the interview protocol unanswered.

This study has several limitations. Primarily, no generalisations can and should be made in regard to the entire population of victims for the purpose of sexual exploitation. As a result, the conclusions should be seen as a report of exploratory rather than explanatory research. In addition, all victims of trafficking that were included in this study were approached through 'gatekeepers', institutions, and organisations that have somehow been in touch with them. Thus, trafficking victims that have not been officially identified as such, or have not had any interaction with official bodies, were not captured in the sample. In addition, conclusions regarding the success or failure of the recovery and (re)integration process in the long run were drawn based on input from service providers, primarily. More information from women themselves would be necessary to make any significant generalisations.

Nonetheless, the value of service providers as sources of knowledge, with extensive and thorough experience with human trafficking victims should not be underestimated. A greater focus on them as respondents instead of trafficking victims may help avoid situations of re-victimisation of persons trafficked through the interview process. Of course, this would not solve the 
problem of failure to capture those victims that are never identified as such or who decline services.

Finally, human trafficking research continues to require innovative methodologies and approaches. Personal diaries (explained in more detail in Chapter 8: Conclusion) of victims was an approach that was attempted in this thesis but unsuccessfully. Still, it is the determination of the researcher that if successfully implemented this method may result in wealth of information, while avoiding some of the pitfalls of interviews - such as creating unrealistic expectations from the side of the respondent, or failures to build trust. The outlet may also serve as a beneficial coping mechanism for the victim herself. 


\section{Chapter 4: How (re) integration success and (re) integration failure is conceptualised in different contexts for women trafficked for the purpose of sexual exploitation ${ }^{9}$}

\subsection{Introduction}

The primary goal of this thesis is to determine the factors that influence the short-term and long-term (re) integration process of women that have been trafficked for the purpose of sexual exploitation. To do so however, requires defining what successful (re) integration is and what can be considered a failure of the process.

It begins by looking at existing definitions of (re) integration, in the context of human trafficking research. One of the most widely accepted definitions of (re) integration in the context of trafficking states that (re) integration is the "process of recovery and economic and social inclusion following a trafficking experience" (Surtees, 2010, p. 24). Some of the elements of the process are seen to be "settlement in a stable and safe environment, access to a reasonable standard of living, mental and physical well-being, opportunities for personal, social and economic development and access to social and emotional support" (Surtees, 2010, p. 24). What is identified as the key ingredient of successful (re)integration "is that of empowerment, supporting victims to develop skills toward independence and self-sufficiency and to be actively involved in their recovery and (re)integration" (Surtees, 2010, p. 24).

Several points can be made on the basis of this definition. Firstly, recovery is seen as being part of the overall (re) integration process. However, what exactly recovery means in the context of trafficking is not further clarified. Secondly, there seem to be several dimensions of success that can be derived from this definition: (1) settlement in a stable and safe environment, (2) reasonable standard of living, (3) mental and physical well-being, (4) opportunities for personal, social and economic development, (5) access to social and emotional support, last, but most important (6) empowerment, independence, self-sufficiency. However, when reviewing these different aspects of a successful (re) integration, one would then inevitably ask, does

\footnotetext{
${ }^{9}$ This chapter will be submitted for publication as an individual paper
} 
success constitute positive developments along all these dimensions? What if a victim lives in a stable and safe environment, and has social and emotional support, but is not 'independent' - does this constitute success? How realistic is it to expect success along all the noted dimensions, namely, does this definition reflect the real situation in practice? And what would be considered a definitive failure of the (re) integration process?

This chapter aims to answer the above raised questions and compare the existing definitions with practice through research conducted with service providers that work directly with women who have been victims of trafficking for the purpose of sexual exploitation. This chapter is based on the answers provided by service providers when asked what they consider to be successful (re) integration, and what they consider to be a failed (re) integration process, as well as examples of each from their own experience. The main body of this chapter will report on the different dimensions of success of the (re) integration process, as identified by service providers through their work. It will also discuss what is considered to be a failure of the process of (re) integration in practice. In the discussion section, the findings will be contrasted with already existing definitions. In addition, ahead of presenting the empirical results of this research, the concept of recovery in the context of (re) integration following a trafficking experience will be clarified, using existing literature from the field of psychology.

The type of trafficking this chapter focuses on is for the purpose of sexual exploitation, and the victims discussed are women. However, the source of information is service providers that work directly with women that were trafficked for the purpose of sexual exploitation. It should also be said that based on the results that will be presented, it is clear that service providers may confuse outcomes of the (re) integration process with factors that influence the (re) integration process. Outcomes of the (re) integration process will be the primary focus here and will be clearly listed at the end of each section of the main results. In order to clearly distinguish outcomes from influencing factors, the outcomes identified will be contrasted with the means necessary for achieving those outcomes. Finally, although service providers did note that some personal characteristics of victims are seen as elements of a successful (re) integration process, they will not be discussed here, but solely as contributing factors in the following chapter.

The importance of this chapter is in trying to bring together existing definitions closer to actual practical outcomes of (re) integration processes 
and discuss what can be considered a success and failure of the (re) integration process based on reports from the field, rather than focusing on ideal case scenarios that rarely materialise. It has clear policy implications as it will reframe how success is perceived and show that focusing and achieving progress along the lines of any of the dimensions of the (re) integration process is significant for the victim. Its additional academic value is in introducing theory from the field of psychology and in discussing the concept of recovery within the (re) integration process of trafficking victims.

The next section begins with a review of the relevant literature. First, psychology literature on the concept of recovery will be introduced. It will be followed by literature on (re) integration in the context of trafficking. ${ }^{10}$ The researcher will refer back to this literature in the discussion section of this chapter.

\subsection{Existing Literature}

\subsubsection{The concept of recovery and the role of resilience}

A multidimensional definition of recovery from psychological trauma, taking into consideration the ecological view of human development, is given by Mary R. Harvey (Harvey, 1996). Harvey criticises existing definitions of trauma recovery which either (1) refer "to the completion of a lengthy psychotherapy, and to the mastery and final resolution of psychological conflicts that may or may not have their origins in traumatic exposure" (Harvey, 1996, p.11) or (2) focus "particularly on post traumatic arousal and on the intrusive symptoms characteristic of post-traumatic stress disorder" (Harvey, 1996, p.11). Meaning, as soon as there are no more flashbacks or nightmares that relate to the traumatic experience, recovery has been achieved. Such an approach in the context of post-trafficking situations would also be particularly troubling, as recovery is an ongoing process that may go well beyond the time period in which the victim exhibits symptoms of PTSS.

Harvey (1996) instead introduces recovery as a 'multidimensional' process characterised by seven outcome criteria: (1) authority over the remembering process, (2) integration of memory and affect, (3) affect tolerance, (4) symptom mastery, (5) self-esteem and self-cohesion, (6) safe attachment, and

\footnotetext{
${ }^{10}$ Only literature specifically on (re) integration of trafficking victims is discussed here. For more expansive literature on (re) integration of returnees please refer to 'Comparative Research on the Assisted Voluntary Return and Reintegration of Migrants' by Khalid Koser and Katie Kuschminder (2015)
} 
(7) meaning making. Thus, according to Harvey, "recovery is apparent whenever change from a poor outcome to a desired outcome is realised in any one domain affected by traumatic exposure", and further "resiliency is evident when one or more domains remain relatively unimpacted and when the trauma survivor is able to mobilize strengths in one domain to cope with vulnerabilities and secure recovery in another" (Harvey, 1996, p. 14). Harvey's work has been relied upon in current research on the topic of resilience as well, which will be discussed next.

The concept of resilience also requires further exploration as it is the crucial determinant of the mechanism of recovery (Brown et al., 2011; Masten, 2007; Tummala - Narra, 2007; Ungar, 2013; Wright et al., 2003). According to Masten (2007), "resilience is a very broad idea referring to the capacity of dynamic systems to withstand or recover from significant disturbances", in other words, resilience is the capacity to adapt following a shock to the system. Tummala - Narra (2007) reiterates this definition of resilience but points out that we cannot study and look at resilience out of context, nor as a static characteristic, but as one that changes and develops with time. She notes that resilience from a cross cultural perspective "involves examination of multiple phenomena, including individual development, community impact, and cultural systems of thought", and "while both individual attributes and developmental transitions are important contributors to resilience and coping, both are also influenced by salient qualities of family, social support network, and community and by prevailing cultural beliefs and values" (Tummala Narra, 2007, p. 36). Thereby the concept of 'collective resilience' is introduced, and family support as one example of this form or resilience. Family support is a crucial factor in the process of recovery for trafficking victims as well.

Ungar (2013) says that positive functioning can occur in the presence of trauma related symptoms such as PTSD. He also introduces a social ecological definition of resilience and "rather than defining resilience as the individual's capacity to succeed under stress", he defines resilience as "the capacity of both individuals and their environments to interact in ways that optimize developmental processes" (Ungar, 2013, p. 256). According to Ungar (2013) the "ecological definition purposely de-centers individuals to avoid blaming them for not flourishing when there are few opportunities to access resources" (Ungar, 2013, p. 256). So, the study of resilience, and what we take to be indicators of resilience, depends on context and culture. In the context of post-trafficking situations, access to resources is particularly 
troubling when women come from villages, smaller towns or small cities. The problem in these situations is not only that resources that could help recovery are not accessible (usually available in larger cities or capitals) but also the stigmatisation that may follow if a person were to expose their experiences to the community. Of course, when even small communities are supportive rather than judgemental of the victim, this can have a particularly positive influence on recovery (as was the case with one of the respondents in this research).

In Wright et al. (2005), resilience is approached by looking at multiple domains of functioning of mothers that have been victims of sexual abuse as children. The domains are (1) the intrapersonal (absence of depression symptoms and perception of positive physical health), (2) the interpersonal (marital satisfaction) and (3) the intrafamilial (perceived parenting competence) (Wright et al., 2005, p. 1177). They found that while their target group showed resilience in one domain, it may not show resilience in another. Only $16 \%$ of the 79 persons sample showed resilience across all domains. But $81.9 \%$ of the mothers showed resilience in at least one domain. The authors also found that women who had spousal support, although they may have been depressed, functioned better in their role as parent. This is again consistent with some of the cases in this research where for instance one respondent showed symptoms of PTSS but still functioned fairly well in her role as a parent, and sustained a good relationship with her own mother.

Finally, Nicola et al., (2012) look at the characteristics of early recovery from complex trauma of 20 participants, engaged in treatment in an adult outpatient clinic which focuses on care of chronically and complexly traumatised survivors. The data was gathered through in-depth interviews focused on trauma and resilience. The authors find 3 themes 'preludes to vulnerability', 'corruption of self-experience' and 'paradoxical extremes of functioning'. The 'preludes to vulnerability' theme captured the experiences from childhood and/or family experiences, that set the stage for later vulnerability and possibly abuse. The second theme of 'corruption of self-experience' and identity development captured struggles in early recovery - for instance not trusting anybody, or trusting too easily and quickly, reckless behaviour that could physically hurt them, such as drinking too much, stealing, feeling suicidal, and a negative self-image, 'deficient or damaged' - internalisation of the perpetrator's image of them. The second theme also included disparaging talk about their body, as well as either sexual rigidness or sexual frivolity. And the last theme, 'the paradoxical extremes of functioning', focuses on 
nonlinear paths and efforts toward recovery - for instance, getting a wanted job, then burning out. These types of extreme functioning were explained through what is called 'compartmentalised adaptations to significant abuse' that can be internal and external. External are of particular interest for the context of trafficking victims, as they report of a 'split existence', 'having a successful work or school life but engaging in self-destructive behaviour (Nicola et al., 2012, p. 107). Thus, once again, as in the work of Wright et al., it seems that persons may show success along the lines of certain recovery dimensions, and failure along the lines of others. But still, overall, they continue to 'function'.

Thus, how can the literature reviewed, be applied to the process of recovery in the context of trafficking for the purpose of sexual exploitation? Recovery in the context of trafficking should be seen as a process that is a part of the wider (re) integration effort and begins with the start of the (re) integration process and may last as long as the (re) integration process lasts. Recovery from a trafficking experience may have two distinct aspects - physical recovery and psychological recovery. Physical recovery has to do with recuperation from the physical injuries suffered throughout the trafficking experience and will be touched upon more extensively in the following chapter that discusses the dynamics of the (re) integration process following identification as a victim of trafficking. Psychological recovery in the context of trafficking can be seen as having two dimensions individual and social. Individual dimension of psychological recovery would have to do with (1) having authority over the remembering process, (2) self-esteem and selfcohesion and (3) meaning making (Harvey, 1996) - again, more touched upon in the following chapter, as they have to do with personal characteristics of the victim, and finally, (4) recovery from PTSS (post-traumatic stress syndrome). The social dimension of psychological recovery however would be particularly significant here, as it would have to do with the ability of the victim to form safe attachments, meaning new healthy relationships (Harvey, 1996). ${ }^{11}$

\subsubsection{The concept of (re) integration}

This chapter began with a review of one of the key and most comprehensive definitions of what successful (re) integration is, offered by Rebecca Surtees. This section expands on available definitions of (successful) (re) integration.

${ }^{11}$ Please refer to Figure 1 
Bearup (2015) critiques the key definition given by Surtees, by pointing out that the provided definition is overly focused on 'empowerment' and 'selfsufficiency' of victims, therefore not taking into consideration the cultural context, where such elements would not be acceptable. Bearup instead puts particular emphasis on the concept of 'acceptance' by the society to which a person returns, as a key element in achieving successful and sustainable (re) integration (Bearup, 2015). However, what 'acceptance' means depends on whether a woman goes back to a 'traditional-rural' or a 'modern-urban' context. Further, Bearup points out that in the traditional-rural context, acceptance is achieved through adopting a "traditional social role within family and village community", while in the modern-urban context acceptance is achieved through "intimate relationships, market-based associations and engagement within the public sphere", which requires a "more individualized reflexive capacity" (Bearup, p.120).

Pandey et al. (2013) use the definition of (re) integration as provided by the European Council on Refugees and Exiles in their study on the (re) integration process of trafficking victims in India. The definition states that "integration (and reintegration) implies long-term and multi-dimensional states of either integrating into a host country (or reintegrating into a home country setting), which are not achieved until the individual becomes an active member of the economic, cultural, civil, political life of a country and perceives that he or she has oriented and is accepted" (Pandey et al., 2013, p. 53). Pandey et al. (2013) find that the only way in which (re) integration of victims of trafficking for the purpose of sexual exploitation can be successful is when the causes of trafficking are addressed. What is meant by this is the situations of poverty that women who are trafficked often come from. Thus, due to economic need, even when women are identified as victims and (re) integration efforts are made they may choose to return to situations that put them in danger of retrafficking, because it is the only way for them to survive financially. Finally, many of the noted definitions of what (re) integration means for trafficking victims are criticised based on the reality that some trafficking victims were never truly integrated in their communities in the first place and additionally, even if they were to return to the situation of before, that may simply not be possible or even desirable in certain cases (Asquith \& Turner, 2008).

The above presented literature will be revisited in the discussion section of this chapter, after the presentation of the main empirical results of this research. At this point, it should be said that this chapter will not decide on a clear definition of what successful (re) integration is, based on the provided 
review above, nor on the results of this research. The outcomes, or dimensions according to which success can be evaluated, will be discussed. However, instead of a clear definition of success, the focus will be on a continuum of success. It is also the explicit intent of this research not to discuss in detail the distinctions between what could be identified as small progress as oppose to full success of the (re) integration process. The success of the (re) integration process is a continuum, very much dependent on the initial starting point.

\subsection{Methods}

The present research was conducted in Albania, Bosnia Herzegovina, Bulgaria, Montenegro, Serbia, the Netherlands, and Italy. A total of 40 interviews with service providers were conducted, ranging from 20 minutes to over 2 hours in length. Interviews with service providers were mostly conducted in offices of assistance organisations.

Service providers interviewed for this chapter were from Albania (11), Bosnia Herzegovina (5), Bulgaria (5), Italy (1), Montenegro (2), the Netherlands (6) and Serbia (10). Educational background or organisational role of those interviewed was that of case manager (2), children center coordinator (1), clinical social worker (1), crisis unit operator (1), day center coordinator (1), NGO or government agency director (4), member of female NGO lobby (1), hot line operator (1), government worker (1), lawyer (2), police official (2), program manager (3), project coordinator (1), psychologist (7), psychotherapist (1), shelter coordinator (2), shelter staff (3), social worker (2) and course teacher (1).

The interview protocol used for service providers, covered a variety of questions. ${ }^{12}$ Service providers were asked about their daily work and tasks; about how they come in contact with trafficked persons (how victims are identified); what is their initial approach when they come in contact with trafficked women for the first time (what are the needs of the women, what do they want, what do they tell the service provider, how does the service provider interact with them); what the challenges are during these initial contacts; how interactions proceed as time goes by (how do the needs and wishes of the trafficked women change with time; how long do service providers follow up with each case, what are some of the challenges in later interactions) and what according to the service provider constitutes successful

${ }^{12}$ Full interview protocol can be found in Annex 2 
(re) integration (with examples given of successes and failures of the (re) integration process). This chapter is primarily based on the answers given to the last noted question, namely, what constitutes successful (re) integration, accompanied with examples of successes and failures from their experience.

\subsection{What are the elements of (re) integration success?}

Interviewees were asked what they considered to be successful (re) integration, as well as to give examples of successes and failures of the process that they have experienced throughout their work. Based on the responses and results, several groups of elements of successful (re) integration can be identified: economic elements, professional assistance and institutions, and social relationships.

\subsubsection{Economic elements}

Respondents noted that having a house (although it was not clearly stated if this meant owning a house or simply a place to live), the possibility to continue education if this is the wish of the woman, a job or an environment that would give the woman some kind of financial stability and sustainability, as important elements of success. However, there was some contradiction in responses as to whether the woman should be self-sufficient or rely (partly) on help from a partner and/or family. One interviewee defined (re) integration a success:

"if she has let's say a job, or a micro business and she is capable to provide her own income, so to afford her living cost, and not to be fully dependent on her husband, so she is economically empowered. "(Service provider, Albania, 23 October 2015).

However, another respondent noted:

"A lot of them stay, according to a lot of criteria, at the border of some kind of poverty, in the sense of income. But still, they have, we have to measure success, in the sense that now, financially, together with the partner, or in a community, she has some kind of sustainable financial circle. It may be through social benefits too, but also through some kind of work, formal and informal."(Service provider, Serbia, 29 October 2014).

Thus, the economic elements of a successful (re) integration are (1) having a place to live, and (2) having financial stability and sustainability. The 
disagreement lies as to the means through which these economic goals are achieved. In some situations, such goals may be reached through the efforts of the victim (if she is financially independent) or through the efforts of her support system such as her family (if she is financially dependent). It is clearly portrayed through the above noted first quote, that service providers put emphasis on terms such as 'economically empowered' and seem to show preference for the 'economic independence' of the woman. However, this research found that, where the family environment was a healthy one, cases of (re) integration were not called a failure if the woman was financially dependent on her family. Thus, the question may be raised, how much does this emphasis on 'economic empowerment' and 'economic independence' reflect the reality in the field, and how much does it reflect a preference for certain terminology that in some cases may not be in the best interest of the woman. If the woman makes the autonomous decision to be financially reliant on her partner, her relationship is a healthy one built on mutual trust, would this emphasis on independence and 'economic empowerment' make her insecure in her own choices, and doubt her self-worth, which in turn may reverse an otherwise sustainable and successful (re) integration process? This point will be briefly elaborated on in the discussion section of this chapter, contrasting independence to autonomy in the context of (re) integration. However, as this pertains to the means for achieving financial stability and sustainability, it is thus a factor influencing (re) integration rather than an element of successful (re) integration and will be more extensively discussed in the following chapter.

\subsubsection{Professional assistance, institutional and legal empowerment}

In certain cases, respondents noted that the cooperation of many different actors, from a variety of institutions was necessary in order to bring about a successful and sustainable (re) integration process. Speaking of a success case, one respondent noted:

"I have an example of a mother with child. She was here [in the shelter] for a long time. And who couldn't rehabilitate, because of safety reasons. The state helped. There were re-locations, the prosecution, the inspectors, they helped a lot. We moved them so many times, from one place to another, because the safety was compromised. The Ministry of Human Rights, of Security, the people that work there, other NGOs, everyone helped. Its one shining example...we had a lot of mistakes in the process, from which we learned, so we had many mistakes. But we also had so many positive things, that at the end, they were re-located in a third country. And I know they are 
The key factor that made this example a success case of (re) integration was institutional support and inter-institutional cooperation in initially keeping the victim and her family safe and thereafter assisting her in beginning a new life in a new environment. In many situations that were encountered throughout this research, it was not uncommon that victims were kept in shelters or safe houses while the police investigated their trafficking case or were in pursuit of the trafficker. However, in this particular situation, institutional support went beyond concerns about safety during an investigation or trial and succeeded in creating a sustainable life for the woman and her family beyond the reach of her traffickers. It is not clear how often this occurs in practice, but when it does, it is evident that institutional support was crucial for securing and sustaining a successful (re) integration process.

In addition to coordination and cooperation amongst different actors, participation in occupational trainings offered usually by NGOs was also considered as an important element of a successful (re) integration since such participation gave tools for future job search. An established relationship either with a service provider who the victim came to trust, or with a peer another woman that has gone through the same experience, are two additional aspects of professional assistance. Finally, knowledge sharing and assistance given by service providers was seen as important in keeping women informed about their rights, but also making it possible for them to become, what service providers referred to as 'full citizens', and thus, more 'empowered' than they were before trafficking occurred. When speaking about this topic, service providers noted that it is not uncommon for trafficking victims to lack basic documentation such as birth certificates and IDs (service providers saw this as not being a 'full citizen'), which in turn may be necessary to be able to receive health cards, which are crucial for receiving any kind of health treatments. Access to medical treatment, whether related to issues connected to the trafficking experience or not, was seen by service providers as a basic right of the victims in these cases. Thus, providing assistance in securing documentation that would ensure that women could practice this basic right, was seen as an important element of the (re) integration process. The possibility to visit a dentist or a doctor, and in some cases women were encouraged to do so, as part of the (re) integration process meant for service providers that the woman was beginning to take greater care of herself, and thus showed more commitment to staying out of potentially dangerous 
situations. Although not explicitly stated by all respondents, it is believed that in these cases, service providers were often speaking of women belonging to the Roma community.

As one responded stated:

"Because in essence, if we are talking about the cause, as to why someone became a victim of trafficking, then we can ask if that person in the first place had the rights, constitutional, legislative rights that belonged to her, in the country in which she was born. Was that person integrated, and recognised as a citizen, of first, second, third, fourth, fifth order. Then, we come to the issue of integration, (re) integration. It's a fact that most of our clients, did not have the same rights, were not included in all the systems, in the same way. They were not included in the education system, in the health system. They were invisible as subjects, as legal beings. "(Service provider, Serbia, 29 October 2014)

Thus, the institutional elements of success are the (1) institutional functioning, coordination, cooperation in offering support (2) relationships built with service providers and peers (3) legal empowerment in acquiring documents which give access to further support and additional services. Some of the means through which these outcomes can be achieved is building a relationship with victims based on trust from the very first moments of contact, as well as continuous provision of information to victims, which will be discussed in greater detail in the following chapter. Exchange of information between different institutional actors is also an important means through which to achieve the above stated goals.

\subsubsection{Functioning social relationships}

Having family support and a social network to fall back on has been identified as one of the crucial elements of successful (re) integration. In certain cases, it can be the family of origin, where the trafficking survivor either comes back and is welcomed back or is successfully reconciled with the family and is thereafter offered crucial support to sustain their recovery and (re) integration. In other instances, it is considered a success when the victim forms their own family. In fact, being married has been identified as an important element of a successful (re) integration by many respondents. As an interviewee noted:

"Yes, well, I think that when a person... I am glad when they "marry well". It seems to me that for some girls, who are so alone, if they find a good 
companion of life, they make a family, and so: the job is not enough, the job is not enough." (Service provider, Italy, 30 December 2015)

One of the issues that comes up concerning family relations is whether or not victims are open about their trafficking experience with those around them. Some women choose to not disclose their past experiences and continue to have functioning family relationships. It was noted, however, that there are cases when the woman does choose to share her past with her family and sometimes this makes the family unit even stronger:

"But sometimes you know, sometimes the shock that they have lived, the trafficking, the exploitation, sometimes this makes the family stronger, when the family is not involved in the trafficking." (Service provider, Bulgaria, 12 October 2015)

Thus, the social elements of a successful (re) integration are (1) reconciliation with the family of origin or the family created before the trafficking experience and (2) creation of a new family. Briefly going back to the means necessary to achieve this, two conditions would have to be fulfilled for the above outcomes to materialise - first, that the victim regains her ability and wish to re-build or create new relationships and second, the willingness of the family members to form relationships with the victim. It is in this context that service providers spoke about whether or not victims reveal their past to their families and those closest to them. It seems that what is a sign of a healthy relationship is not whether or not the past is revealed, but that the decision to reveal or conceal the past is made solely by the victim. Thus, whether or not the past of the victim is known to those closest to her does not seem to have a clear influence on her ability to form relationships. But, what does seem to have influence is that she is the one who makes the decision to disclose or not. Once again, as this pertains to the influencing factors, it will be discussed in greater detail in the following chapter.

\subsection{A continuum of success}

So far, various economic, institutional, and social elements of a successful (re) integration have been noted and briefly related to the means necessary to achieve them. However, the next question to ask would be, for (re) integration to be marked as a success, do all of these outcomes need to be achieved? Based on the results of this research, the answer would be no. In fact, rather than speaking of successful (re) integration along each element discussed, we 
should be speaking about the continuum of success. And even when (re) integration succeeds along some dimensions, but fails along others, the overall process may still be seen as satisfactory, in practice.

For instance, respondents noted that women with some addiction issues may be considered success cases. As one interviewee pointed out:

"Yes, for instance, what is successful (re) integration? We have a woman that was for years a heroin addict, has Hepatitis etc. She speaks 4 languages, works in a foreign firm, makes much more money than all of us combined. She drinks. So what? That is nothing, compared to what she was in the past. If you ask me, it's a triple success. She went through rape, through the life of an addict, drugs... and from that into human trafficking. Then, she went into drug addiction therapy. She was on different types of drugs, over 3 years. Now, finally, she found employment, she is working.... she drinks. But so what? She is functional, every day she is at work. She is doing very complex tasks, she has knowledge, she cooperates, lives normally. She has no connection with the people of the past. If you ask me, that is successful (re) integration." (Service provider, Serbia, 2 November 2015)

Thus, it seems that in measuring and looking at success, we should clearly consider the starting point. When a person comes from a severely disadvantaged position, independently of the trafficking situation, and has made such economic progress that would be considered for any individual a move up the social ladder, the outcomes outlined above should be considered as significant success. In this context however, the progress that she has made comes with the tradeoff of showing dependency on alcohol. This was clearly stated by the respondents:

"Successful (re) integration? Well, when I began working, I thought that I have to satisfy a lot of needs, to call a situation successful, that the quality of life should be significantly better. So someone that has no education, has to be enrolled, etc. But with time, I realised that the capacity for change, is not the same in everyone. And that not everybody has that need, that we often have in the head, that they have to be like this, this and this, and that they have to live a perfect life, without problems. I understood that even a small step of progress, makes their life better, and betters their quality of life." (Service provider, Serbia, 3 November 2015) 
The same interviewee further noted in regard to addiction that:

"with time, I learned that when we have a collection of bad factors, if the person is an addict, someone that does not have good contact with family, no education, no job, no place to live, no basic subsistence. I decrease the demands, in front of myself, and also in front of the person. When we do even small steps, then I am happy with the person and myself. At the beginning, I was always putting very high goals, and then I would be very unsatisfied, myself and also the victim. I would feel a failure. But now, I can see the risk factors, the strengths, the potential for which I can get a grip. I need to do a good diagnosis, evaluation."(Service provider, Serbia, 3 November 2015)

During this conversation, the above respondent remarked that when she began working in this field, she would actually have had the same very high expectations for each of the persons that she encountered, but high expectations as to what is desirable in her opinion. Thus, she would have been disappointed if every person she took on would not have continued or completed their education. In the period of the interview, the organisation was actually dealing with the loss of life of one of their beneficiaries. The person was a victim of trafficking and a former addict that was recently doing very well, but her body had suffered too much in the past, so although the exact cause of death was still under investigation, it was assumed that it was due to past physical traumas. Thus, taking all of this into consideration, in certain cases, finding ways of keeping the person alive, away from drugs, and out of situations of exploitation, would be considered a success.

According to other service providers, a successful (re) integration is:

"To be able to say no, to things that are not fitting to her. To be able to make choices that according to some standards could be good or bad. That she does not enter into, that is, that she recognises violence, not just physical, but also in the sense places that make her feel good, and make her feel bad. So she is able to stay away from violent relationships. "(Service provider, Serbia, 29 October 2014)

"That in a way, they get back to normal life, normal things in life. That they stand on their own two feet. That they connect with those people, whether its family or friends, that are for them real support. And not find people who may again introduce them to some problems. To continue with education, if education had been interrupted. Or continue further, in case they had finished 
high school. Maybe they should go to university. To find a job, to start living independently. Not to live in a situation of violence. To have some healthy, functional relationships. Whether with romantic partners, with family or something similar."(Service provider, Serbia, 30 October 2014)

As one interviewee summed it up:

"What I think is a successful case is sometimes a miracle because you never can...It is very rare that you can reach these...It's a woman that can have her autonomous and independent life, without violence."(Service provider, Bulgaria, 9 July 2014)

\subsection{Failed (re) integration}

In the previous section it was stated that the (re) integration process may be considered a success, even if a woman is still for instance struggling with issues of addiction. In fact, drug and substance abuse problems were often mentioned by service providers as some of the most significant challenges for victims, following a trafficking experience (that may or may not be a direct consequence of the trafficking - as in some cases, addiction issues predate the trafficking experience). But nonetheless, addiction was not seen as an outcome which would mark a (re) integration process a failure. However, it should be noted that this reference is to alcohol addiction only. Drug addiction, which as described in one of the paragraphs above, could lead to loss of life and would not in any situation be considered a success. In addition, outcomes such as permanent institutionalisation and continuous dependence on institutional help by certain victims dealing with mental challenges, were also not seen as a failure of the (re) integration process. An example was given of a victim with significant mental challenges that was permanently placed in an institution, as she was seen as incapable of living independently. However, although institutionalised, it was recommended that she retain custody of her underage son, who was to live with her in the institution. This was not considered a failed (re) integration process.

Thus, this leads to the question, what is the bottom line outcome which if present, would clearly signal a failed (re) integration process? Based on this research, a failure of the (re) integration process is when a woman would return to a situation of exploitation, when regression or re-trafficking occurred and the woman was again under threat or in a situation of violence and exploitation. It was in this context that some service providers would note 
that they strongly discourage return to sex work, as it would again place the victim in potential situations of exploitation - physical, mental abuse or financial exploitation.

\subsection{Discussion}

The overall (re) integration process, of which recovery is a part (as discussed at the beginning of this chapter), has 3 additional distinct dimensions along which success can be measured, which correspond to the empirical findings of this chapter. The following dimensions have been identified as a result of this research. All of the dimensions already discussed throughout this Chapter, thus fall within the following 3 elements and their subgroups. Economic dimension, that has to do with (1) having a place to live, and (2) having financial stability and sustainability; institutional dimension: (1) institutional functioning, coordination, cooperation in offering support (2) relationships built with service providers and peers (3) legal empowerment in acquiring documents which give access to further support and additional services and finally social: (1) reconciliation with the family of origin or the family created before the trafficking experience and (2) creation of a new family. However, success in regard to the social dimension of psychological recovery would mean the ability of the victim to form safe attachments, whereas success in regard to the social dimension of (re) integration would mean acceptance of the victim from the side of the family, whether family of origin or newly formed. Thus, social psychological recovery is from the perspective of the victim and falls within the wider umbrella term of overall (re) integration, whereas social (re) integration is from the perspective of the 'others', which most often in the case of trafficking victims, is family members. 


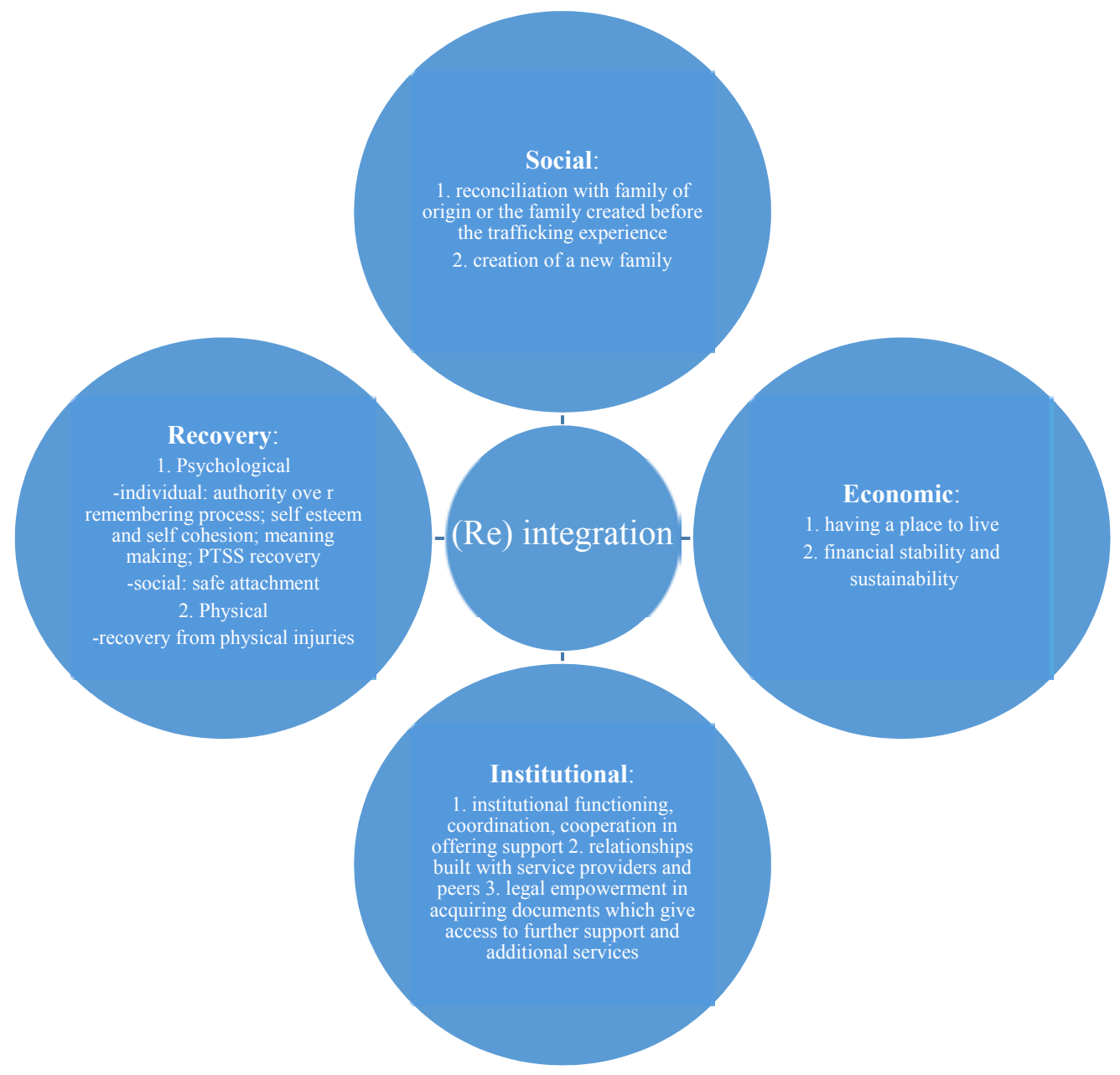

Figure 2: Elements of the (re)integration process

The elements of (re) integration described in Figure 2 can additionally be categorised at the micro, meso and macro level of the ecological environment conceptualised as part of the ecological model of human development. Additional priorities could also be identified based on the chronology of needs of the victim. In this regard, all subelements of the process of recovery belong to the micro level of the ecological environment, as they have to do solely with activities, roles and personal relationships, from the perspective of the individual. The elements of (re) integration which fall under the social dimension belong to the meso level of the ecological environment, as they entail an interaction of two different settings - that of the individual and her social environment, in particular her interactions and relationships formed 
with members of her family of origin, as well as a newly formed family. Certain elements of the institutional dimension, as well as the economic dimension belong to the meso level as well. Thus, any interaction of the survivor with members of different institutions, the formation of relationships with service providers or peers belong to the meso level. Her success in finding a place to live, as well as a way to sustain herself economically, also belong to the meso level of the ecological environment. However, certain aspects of the institutional as well as economic dimension of (re) integration do not depend on the interactions of the individual with the institutional and economic sphere, but rather on the wider societal context, which places them in the macro level of the ecological environment. Thus, the efficiency of institutions, occurrence of corruption which may prevent their functioning, lack of funding are all aspects which do not depend on the interactions of the victim with the institutions she has access to. In addition, a period of economic and wider political crisis in the country in which she resides are also not dependent on her interactions with these environments, but nonetheless, determine her institutional and economic (re) integration. Certain (re) integration outcomes should be aimed at early on in the (re) integration process. These mainly have to do with the micro level, and the immediate psychological and physical recovery of the victim. Thus, dealing with issues such as PTSS and making sure that the survivor has recovered from any physical injuries would always take priority over ensuing financial stability and sustainability, as well as re-building of social relationships.

Having the overall theoretical framework, the remaining of this discussion will focus on the empirical findings of this chapter. First, it should be said that this is not an exhaustive list of elements of success. These are simply the outcomes that were identified by service providers when defining what they see as successful (re) integration. These particular outcomes are not contrary to the outcomes identified in existing definitions of successful (re) integration. As already noted, Surtees (2010) identifies elements of success such as living in a stable and safe environment, a reasonable standard of living, access to emotional and social support, and opportunities for personal, social and economic development.

However, where the findings of this chapter diverge from current definitions of successful (re) integration is in stating that success does not necessarily mean positive outcomes along every dimension identified. And in this regard, the findings here are more relatable to the literature on recovery (Brown et al., 2011; Harvey, 1996; Masten, 2007; Tummala - Narra, 2007; Ungar, 2013; 
Wright et al., 2003; Nicola et al., 2012). Namely, success can be seen whenever there is progress in regard to any of the dimensions of (re) integration. Thus, instead of speaking of definitive success of the (re) integration process, it is much closer to what happens in practice to speak of the continuum of success along different dimensions. Similarly, what Nicola et al. (2012) coined 'paradoxical extremes of functioning', particularly in the context of recovery from complex trauma, are not uncommon in the context of (re) integration following a trafficking experience. As already noted in some of the examples given, a woman may be addicted to alcohol, but still hold a steady job, which by her service providers was seen as significant positive progress. This, particularly when compared to her initial starting point of being a heroin addict, with various physical health problems, and who suffered rape even before entering a situation of trafficking. Thus, one of the crucial points to be made from this research would be to say that the focus should be on the continuum of success, in particular considering the starting point of many of the victims, rather than demanding or expecting clear success along all dimensions identified as relevant. The one condition, which if present would mark the (re) integration process as a failure, was if a woman was still in a situation of violence and exploitation.

An additional divergence of the current research with existing definitions of (re) integration success has to do with the issue of empowerment, independence, and self-sufficiency, which were identified as key ingredients of a successful (re) integration process (Surtees, 2010). What this research has found to be crucial is autonomy in decision making, rather than independence, issues touched upon here but discussed in great detail in the context of marriage, in Chapter 5. As previously acknowledged, it was not seen as a failure of the (re) integration process, if a woman was financially dependent on her social network - be it her family of origin or her husband. Marriage, rather than a woman being 'independent' was seen as one of the elements of success that gives stability and sustainability to the (re) integration process. The partial explanation as to the divergence of existing definitions of success and their emphasis on independence, with actual practices outlined by this research, where marriage is actually seen as a positive element, have to do with confusion between the concepts of 'independence' and 'autonomy'. According to Ryan et al. (2016), confusion between the two concepts in psychology literature is not uncommon. Where independence means "not relying on others", autonomy implies "willingness or volition" (p. 389). Thus, as the study of Ryan et al. (2016) goes on to say, when a teenager willingly asks for help from a parent, this is considered as an 
occurrence that may positively contribute toward the development of the teenager. Nonetheless, he/she would in this case be making an autonomous decision to be more dependent on another (in this case the parent). Along the same lines, what this research found to be crucial is the autonomous decisions of women to enter healthy relationships, even if those relationships may mean financial or other types of dependence (dependence for emotional support for instance). And in this regard, a new marriage was found by this research to be equally important as healthy relations with the family of origin. Wherever the emotional support, which may translate into financial support, is coming from, the important element is that it is derived from healthy relationships, which women entered into through their own autonomous decisions independence and self-sufficiency in this context were found to be irrelevant.

To summarise, this chapter has framed the overall (re) integration process and outlined where the concept of recovery comes in within this process. Furthermore, based on definitions of successful (re) integration provided by service providers, it has outlined various dimensions along which success should be measured and what should be considered a failed (re) integration process. However, the primary importance has been in showing that we should not be speaking about clear success of the (re) integration process, but rather about the continuum of success along its different dimensions. In this context, it is autonomy, not independence of the woman, that should be seen as a desired outcome of the process. 


\section{Chapter 5: Long-term (re) integration of persons trafficked for the purpose of sexual exploitation ${ }^{13}$}

\subsection{Introduction}

Current assistance programmes monitor the (re) integration progress of their beneficiaries, most often for up to 3 years after their identification as a victim of trafficking (Surtees, 2010). Thus, it is this initial 3 year period, which for the purpose of this thesis will be considered as the short run, that is academically most familiar (the most relevant of this research will be reviewed in the next section of this chapter). Nonetheless, some questions remain, not only in regard to this initial (re) integration period, but also in regard to (re) integration efforts in the long run. Are some factors more important than others, in the short and long run? What are the overall dynamics of the (re) integration process, how do the relevant influencing factors interact? What factors are crucial for a positive (re) integration immediately after the experience, and how do they differ from what becomes important as the years go by? What is crucial in order to ensure a sustainable (re) integration? These are the questions that this chapter will address.

The focus of this chapter is trafficking for the purpose of sexual exploitation, and the target group is women that have been victims of this type of trafficking. The respondents are service providers and victims that come from both origin and destination countries in Europe. This research is relevant, as it focuses on the issues trafficking survivors face in the short term and longterm. In addition to identifying the factors that influence the (re) integration process, it will also discuss the dynamics of the same, and distinguish those that are more from those that are less important. In turn, this could inform policy making and practitioners in the field and orient them toward adequately addressing the needs of trafficked persons immediately after, but also many years after the trafficking experience.

This chapter begins with an overview of literature that will be an important reference considering the research questions. Thereafter the methodology of this chapter will be discussed. The main results of the chapter will be presented in the following sections, reflecting the influencing factors on the

${ }^{13}$ This chapter will be submitted for publication as an individual paper 
(re) integration process namely (1) background of trafficked person (2) trafficking experience (3) role of institutions, NGOs and service providers (4) economic factors (5) personal characteristics (6) role of family and friends. The first 3 sections $(1,2,3)$ have to do with the past of the trafficking victim or are relevant to the initial post-trafficking period. The last 3 sections $(4,5$, 6) are factors that influence the present-day situation of the trafficked person. In the concluding section of this chapter, the dynamics of the interactions of all of the above outlined factors will be discussed, the relative importance of some over others will be noted, and finally, those factors found to be of great importance for the long-term sustainability of the (re) integration process will be identified.

\subsection{Existing Literature}

One of the most comprehensive overviews of the factors influencing the (re) integration process of victims of sex trafficking is offered by Rebecca Surtees through her research and reports on monitoring anti-trafficking re/integration programs (2010) and more recently, research on post trafficking situations in the Greater Mekong Sub-region (2013). Surtees has perhaps provided the most exhaustive list of factors which influence the (re) integration process of women trafficked for the purpose of sexual exploitation to date (Surtees, 2010). The identified 'impact indicators' are 1) safe and affordable accommodation; 2) legal status; 3) professional and employment opportunities; 4) education and training opportunities; 5) security and safety; 6) healthy social environment; 7) social well-being and positive interpersonal relations; 8) economic situation; 9) physical well-being; 10) mental wellbeing; 11) access to services and opportunities; 12) motivation and commitment to the reintegration process; 13) legal issues and court proceedings; 14) assistance to secondary beneficiaries (family members) (Surtees, 2010). In her study 'After trafficking experiences and challenges in reintegration in the Greater Mekong Sub-region', Surtees outlines the challenges to the (re) integration process, which often have to do with assistance provided (or not provided to victims), lack of individualised support, lack of accommodation, legal and administrative issues, economic challenges, poor physical and health well-being, psychological challenges such as stress, anxiety, depression and trauma, being unsafe and insecure, and finally, the many challenges when working with families of victims of trafficking (2013). She does note that (re) integration is a long-term process and where it is possible to conduct monitoring of individual cases, this may be beneficial to the victims. 
However, questions still remain about the particular dynamics and interactions of identified factors that influence (re) integration processes. In addition, more research is needed in the direction of factors that contribute to the sustainability of the (re) integration process. It should be noted that some studies particularly focus on the importance of the family in the (re) integration processes of women after a trafficking experience (Brunovskis \& Surtees, 2012), However, the primary focus here is mostly on (re) integration dynamics that occur when a woman goes back to family that she had formed before the trafficking experience, or the (re)integration dynamics for children born while the woman was in a trafficking situation. The role of families formed after a trafficking experience remains to be explored in greater detail. This research is one small step in that direction.

Jyoti Sharma (2015) examines the issue of (re) integration of female sex trafficking victims in Nepal. The focus is on exploring how women who are survivors of sex trafficking experience the process of (re)integration, and thus, identifying the barriers to (re)integration as well as the supporting factors which promote (re) integration. What is of particular importance concerning this research is that through the experience of fieldwork, it is concluded that (re) integration is an 'ongoing process that can span decades' and thus, the time distance from the trafficking experience for the women on which this study is based ranges from one month to 20 years (Sharma, 2015; p. 45). Some of the identified barriers to reintegration are the social construction of gender in Nepalese society, negative community attitudes, physical and emotional health problems, and the NGOs' lack of a victim centered approach to (re) integration. Factors which support the process of (re) integration are family support, increased autonomy, resilience, and independence (although it should be noted these factors were more relevant for women that chose to leave their village of origin and settle in Kathmandu). However, Sharma does not clearly distinguish between factors that are important in the short term and factors that are important in the long-term, which will be the contribution of this chapter.

Overall, stigma and social discrimination by the family and community are overwhelmingly distinguished as some of the primary challenges to (re) integration of women that have been trafficked for sexual exploitation (Gjermeni et al., 2008; Brunovskis and Surtees, 2012; Gan et al., 2014; Dahal et al., 2015). Additional factors identified as influencing the (re) integration process are financial instability and lack of economic and job opportunities, as well as health and mental problems (Gan et al., 2014). Resilience has been 
identified as a personal characteristic of trafficking victims, which aids the process of (re) integration (Sharma, 2015; Le, 2016). Finally, marriage has been noted as a factor which contributes toward the sustainability of the (re) integration process (Crawford and Kaufmann, 2008; Sharma, 2015; Le, 2016).

One factor that influences the (re) integration process and should be singled out is the issue of trust, as identified from the work of Surtees, but also from the work of Walsh, Black \& Koser (1999) and Koser \& Pinkerton (2002) on repatriation of refugees to Bosnia Herzegovina in the 1990s and 2000s. Trust in information that trafficking victims or returnees receive from the service providers that are in touch with them throughout the (re) integration process, as well as trust in the service providers themselves. Brunovskis \& Surtees (2012) identify trust as crucial in decisions victims make as to whether or not to accept assistance throughout the (re) integration process. The work of Walsh, Black \& Koser (1999) and Koser \& Pinkerton (2002) on the other hand focuses on the lack of trust in information that returnees receive from government agencies and the trust they have in information received from migrant and refugee community organisations. This is a factor that comes through in this research as well, relevant for both the short and long-term (re) integration of the trafficking victim and will be discussed in detail later on. Table 2 gives a comprehensive overview of the above discussed literature. 


\begin{tabular}{|c|c|c|}
\hline Authors & Focus & Chapter 5 (this research) \\
\hline Surtees, 2010 & $\begin{array}{l}\text { identifies impact indicators (2010): 1) } \\
\text { safe and affordable accommodation; 2) } \\
\text { legal status; 3) professional and } \\
\text { employment opportunities; 4) education } \\
\text { and training opportunities; 5) security } \\
\text { and safety; 6) healthy social environment; } \\
\text { 7) social well-being and positive } \\
\text { interpersonal relations; 8) economic } \\
\text { situation; 9) physical well-being; 10) } \\
\text { mental well-being; 11) access to services } \\
\text { and opportunities; 12) motivation and } \\
\text { commitment to reintegration process; 13) } \\
\text { legal issues and court proceedings; 14) } \\
\text { assistance to secondary beneficiaries; }\end{array}$ & $\begin{array}{l}\text { All the noted impact factors will } \\
\text { be discussed throughout this } \\
\text { research as well. However, a } \\
\text { distinction will be made as to } \\
\text { what is more relevant in the } \\
\text { short versus long run, and what } \\
\text { is overall more important what } \\
\text { less. }\end{array}$ \\
\hline Surtees, 2013 & $\begin{array}{l}\text { identifies challenges throughout (re) } \\
\text { integration process: } \\
\text { 1) assistance provided (or not provided to } \\
\text { victims), 2) lack of individualised } \\
\text { support, 3) lack of accommodation, 4) } \\
\text { legal and administrative issues, 5) } \\
\text { economic challenges, 6) poor physical } \\
\text { and health well-being, 7) psychological } \\
\text { challenges such as stress, anxiety, } \\
\text { depression and trauma, 8) being unsafe } \\
\text { and insecure, 9) challenges when working } \\
\text { with families of victims of trafficking }\end{array}$ & $\begin{array}{l}\text { In this chapter, the focus is not } \\
\text { only on the challenges } \\
\text { throughout the process, but also } \\
\text { on factors that are found to have } \\
\text { a profound positive influence on } \\
\text { the (re) integration process. }\end{array}$ \\
\hline $\begin{array}{l}\text { Brunovskis, } \\
\text { Surtees, } 2012\end{array}$ & $\begin{array}{l}\text { role of families of origin, or families } \\
\text { formed prior to the trafficking experience, } \\
\text { children born prior to the trafficking } \\
\text { experience }\end{array}$ & $\begin{array}{l}\text { This chapter will also focus on } \\
\text { the role of the family that is } \\
\text { formed after the trafficking } \\
\text { experience. }\end{array}$ \\
\hline Sharma, 2015 & $\begin{array}{l}\text { Challenges: social construction of gender, } \\
\text { negative community attitudes, physical } \\
\text { and emotional health problems, NGO's } \\
\text { lack of victim centred approach. Factors } \\
\text { that support (re) integration: family } \\
\text { support, increased autonomy and } \\
\text { independence, resilience. }\end{array}$ & $\begin{array}{l}\text { A clear distinction of challenges } \\
\text { and supporting factors in the } \\
\text { short run and long run will be } \\
\text { made, in particular focusing on } \\
\text { what is important for (re) } \\
\text { integration in the long run. }\end{array}$ \\
\hline
\end{tabular}




\begin{tabular}{|c|c|c|}
\hline Authors & Focus & Chapter 5 (this research) \\
\hline $\begin{array}{l}\text { Gjermeni et al., } \\
2008 ; \\
\text { Brunovskis \& } \\
\text { Surtees, 2012; } \\
\text { Gan et al., } \\
\text { 2014; Dahal et } \\
\text { al., } 2015\end{array}$ & $\begin{array}{l}\text { stigma and social discrimination by the } \\
\text { family and community }\end{array}$ & $\begin{array}{l}\text { Stigma around the issue of sex } \\
\text { work has been found to be so } \\
\text { significant through this research } \\
\text { that these factors are the sole } \\
\text { focus of Chapter } 7 .\end{array}$ \\
\hline Gan et al., 2014 & $\begin{array}{l}\text { financial instability, lack of economic and } \\
\text { job opportunities, health and mental } \\
\text { problems }\end{array}$ & $\begin{array}{l}\text { All factors covered in this } \\
\text { chapter. }\end{array}$ \\
\hline $\begin{array}{l}\text { Sharma, 2015; } \\
\text { Le, } 2016\end{array}$ & resilience & Addressed in this chapter \\
\hline $\begin{array}{l}\text { Crawford \& } \\
\text { Kaufmann, } \\
\text { 2008; Sharma, } \\
\text { 2015; Le, } 2016\end{array}$ & marriage & $\begin{array}{l}\text { Discussed in this chapter as well } \\
\text { as Chapter } 4\end{array}$ \\
\hline $\begin{array}{l}\text { Walsh, Black } \\
\text { \& Koser, 1999; } \\
\text { Koser \& } \\
\text { Pinkerton, } \\
\text { 2002; } \\
\text { Brunovskis \& } \\
\text { Surtees, 2012 }\end{array}$ & trust & $\begin{array}{l}\text { Whereas the noted authors } \\
\text { addressed trust either in } \\
\text { information received by } \\
\text { beneficiary from service } \\
\text { provider, or initial trust in the } \\
\text { service provider upon meeting } \\
\text { them, this chapter will focus on } \\
\text { the role trust plays in } \\
\text { establishing a more lasting } \\
\text { relationship between service } \\
\text { provider and victim, that may } \\
\text { have positive influence on long } \\
\text { term (re) integration efforts. }\end{array}$ \\
\hline
\end{tabular}




\subsection{Methods}

The data collection was conducted in Albania, Bosnia Herzegovina, Bulgaria, Montenegro, Serbia, the Netherlands, and Italy from 4 November 2013 to 30 December 2015. A total of 52 semi-structured interviews were conducted. Interviews ranged from 20 minutes to over 2 hours in length. Interviews with service providers $(N=40)$ were mostly conducted in offices of assistance organisations, while interviews with victims were in locations where they felt most comfortable, such as offices of assistance organisations, cafes or in one case, the home of the victim, where the family of the victim (mother, brother and sister) were also present and participated in the conversation. A total of 9 victims of trafficking, 8 female, and 1 male were interviewed, all victims of trafficking for purpose of sexual exploitation. 4 of the victims ( 3 female and 1 male) were from Bulgaria trafficked to the Netherlands, Spain, and an undisclosed country of destination, 2 of the victims were from Albania and trafficked to Italy and Greece, 2 from Romania were trafficked to the Netherlands, and 1 from Bosnia Herzegovina was trafficked internally. Ages ranged from 22 to 42 , while the duration of the trafficking experience ranged from 14 days to 3 years. Although it was not always possible to ask or understand how much time has passed since the trafficking experience, for those victims that made it clear, distance from the trafficking experience ranged from several months to 10 years. In addition, 2 women were sisters, who as young children were identified as 'at risk' of being trafficked, and as such were placed in a shelter until they reached adulthood. Finally, one of the victims from Albania, as already noted, was interviewed at her home, with the presence of her mother, brother, and sister. The sister of the victim also participated in the conversation and was included in the interview.

Service providers were from a range of professions, where the educational background did not always coincide with the current assistance role in the (re) integration process. Overall, 40 service providers were interviewed, coming from Albania (11), Bosnia Herzegovina (5), Bulgaria (5), Italy (1), Montenegro (2), the Netherlands (6) and Serbia (10). Educational background or organisational role of those interviewed was that of case manager (2), children center coordinator (1), clinical social worker (1), crisis unit operator (1), day center coordinator (1), NGO or government agency director (4), member of female NGO lobby (1), hot line operator (1), government worker (1), lawyer (2), police official (2), program manager (3), project coordinator (1), psychologist (7), psychotherapist (1), shelter coordinator (2), shelter staff (3), social worker (2) and course teacher (1). 
Different interview protocols and questionnaires were used for trafficking victims, those at risk of trafficking, and service providers. The questionnaire for trafficked persons covered several general areas, focusing on 1) life before the trafficking experience, 2) the trafficking experience itself; how it happened and what helped the person to get through it, 3) how identification as 'trafficked' occurred, 4) description of the period immediately following trafficking, including questions to do with experience of stigma and participation in assistance programs, 5) description of economic situation at the time of the interview, 6) description of social life, and mental and physical health at the time of interview, finally, 7) description of how the person envisions the future. A selection of the above presented questionnaire areas were used for those at risk of trafficking, omitting any inquiry about the trafficking experience.

A different interview protocol was used for service providers. Service providers were asked about their daily work and tasks; about how they come in contact with trafficked persons (how victims are identified); what is their initial approach when they come in contact with trafficked women for the first time (what are the needs of the women, what do they want, what do they tell the service provider, how does the service provider interact with them); what the challenges are, during these initial contacts; how interactions proceed as time goes by (how do the needs and wishes of the trafficked women change with time; how long do service providers follow up with each case, what are some of the challenges in later interactions) and what according to the service provider constitutes successful reintegration (with examples given of successes and failures of the reintegration process).

The interviews were conducted in English, Bosnian, Bulgarian, Montenegrian, Serbian, and Albanian. For the interviews conducted in Albanian, a professional translator was used, or in certain cases an employee of the organisation that arranged the interviews. The English language interviews were then transcribed verbatim, while the interviews conducted in languages other than English were translated and transcribed directly into English.

The data analysis was conducted with the use of QSR Nvivo 11.2.2 (1707) for Mac. There were multiple levels of analysis. Initially, themes were identified based on the distinctive sections of the questionnaires. The second level of analysis produced in vivo and descriptive codes (types of codes described in Saldaña, 2009) within each theme. The third level of analysis 
entailed designing mind maps based on the themes and codes within each theme, and producing categories on the topics covered within this chapter. The mind maps were further used to draw relationships between categories and connections of codes within each category, and among different codes from different categories identified. All text from the interviews was coded.

\subsection{What factors influence the (re) integration process?}

The various factors that influence the (re) integration process of sex trafficking victims can be grouped according to the time period they belong to in the life of the trafficking victim. Namely, factors stemming from the background of the individual, prior to the trafficking experience, factors related to the trafficking experience, factors having to do with the identification process as well as the role of different service providing institutions, and the factors from the present in terms of economic and social aspects, as well as personal characteristics of the victim. Some factors influence the (re) integration process in a positive way, some in a negative way, and each will be discussed in regard to how they shape the life of the trafficking survivor and determine the presence or absence of violence, exploitation, and possibility of re-trafficking or in other words, in regard to how they determine the success or failure of the (re) integration process.

\subsubsection{Background factors}

There are various ways in which the background of the individual not only influences the (re) integration process at present but may have been one of the causes of the trafficking experience.

The social group to which some women belong has been identified as a possible factor that contributes toward the vulnerability of becoming a victim of trafficking. Respondents noted that women that come from communities which are usually socially isolated, discriminated against, and poor, such as the Roma, may be especially vulnerable to becoming trafficking victims. As one respondent noted:

"It is often that in Roma neighbourhoods, where people that are in social need and at risk live, that according to our experience we find risk groups that may be vulnerable to trafficking (because of early marriage, forced marriage, begging, family violence, and sexual abuse)." (Service provider, Montenegro, 7 December, 2015) 
Another interviewee stated:

"I've seen, especially with the Roma women, in their own country they are looked down upon so much, if they go to a supermarket they are even shut out. For them to be treated as human being is for some of them so exceptional, if their whole life has been a cast way and nobody has never looked at them as a person." (Service provider, Netherlands, 14 March 2014)

It was also found that victims may have come from communities where the business for the girls, from a young age was prostitution. In all such instances, the background of the victim should be considered as a negative influence on the (re) integration process, or in this case, integration process, as it would not necessarily be advisable to seek the return of a woman to her community in these situations.

Migration is another component of the background of women who have been found to fall victim to sex trafficking. Victims interviewed for this study reported falling victim to trafficking when moving from village to city, when moving out of the country of origin in search of employment, or when simply following a partner abroad who thereafter exploited them. However, it should be noted that in all cases encountered, it was not the migration process itself that precipitated the trafficking experience, but rather the unsafe conditions under which it occurred as many women were misled or simply lied to about where they were going, what they would be doing, or the conditions in which they would be doing it. Thus, women even after the trafficking experience did not show to be discouraged from migrating again. As one victim stated to her English language teacher:

"Teacher, I want to learn English, because I see it as very necessary for my life, because I plan in my life to not live here in Albania, but to live outside Albania, and that's why I think it is very useful for me to learn it, that's why I want to learn it, really."(Service provider, Albania, 23 October 2015)

Background economic factors such as poverty and joblessness, which are sometimes related to low levels of education, are also an active influence on the success or failure of the (re) integration process of victims. In this section, what is taken into consideration is the situation of the victim in regard to these issues prior to the occurrence of trafficking, not the situation at present. Namely, it has been found through this research that many of the victims of trafficking had low levels of education or low paying jobs that pushed them 
to seek other possibilities. In these cases, aiming for the return of the victim to such situations or conditions may not have a positive influence on the (re) integration process. As one interviewee noted, while explaining why she would not consider returning to the country of origin:

"I have too much things not nice in past. You know. I was starting to go working, when I was in school I had 10 years, and my mom send me in the work. I had no child [meaning I had no real childhood], and the work, for me was not well. And I said, I want, what I don't have in my past, I want my child to have."(Trafficked person, Netherlands, 13 August 2015)

Respondents also noted having lives filled with problems and struggles to make ends meet prior to the trafficking experience:

"Because I was very very very ugly life. All my life. Was not normal life. I was very much work. I only work, only my mother sick, I have only problems (...)I don't like stress. I think I have problem here. Too much stress, I am not resistant for stress, because when I was young, I was, my family, my parents, my father left when I have 15 years. My father dead. My mother alone gave me ate. Was problem in my family. 4 kids, alone my mother. Only stress. I have never money for what I want. Then I go work, yeah"(Trafficked person, Netherlands, 13 August 2015)

The educational background of the victim of trafficking may be another factor that influences the (re) integration process. There are cases where women who have received only a very rudimentary education then chose to make the continuation of their education one of their goals and aims, which gives them the motivation and energy to go on, thus positively influencing the (re) integration process. However, as some service providers point out, having an education does not guarantee a job and financial stability, thus women should not be pressured to continue their education. In these cases, trainings and specialisations in different professional fields may be of greater importance.

Finally, perhaps the most significant factor from the past that influences the (re) integration process is family background. As already observed, many of the victims come from families with difficult relations and, in some cases, history of violence or situations where the parents are the traffickers. As one respondent noted: 
"Depends on the life they had earlier. There was a girl that was a victim of violence, from her parents, since she was little. She learned that for her, the way you express love is through violence. When someone hits her, it's not violence, because her mom and dad were hitting her, and in that way were showing their love. She connects these things. So, then, when she enters a situation, a situation that was traumatic, she needed some time to get out of that."'(Service provider, Serbia, 2 November 2015)

In another case, a victim had been rejected by the family early on in life:

"First it was mom and dad, until I was 23, I was with my parents. Then, it was stressful, the stress came. My mom came, and she said, I will throw you out of the house, all because of their problems. I said, no, don't fight."(Trafficked person, Bosnia Herzegovina, 1 October 2015).

It is clear that in cases where the victim experienced family violence and abuse, family reunification would not have a positive influence on the (re) integration process of the victim. However, it should be noted that in certain situations, when the victim was not physically abused but emotionally rejected, reunification was still sought after by the service providers as they evaluated that overall, if achieved, it would have a positive influence on the psychological recovery of the victim. Cases were also reported, however, of when the victim was trafficked as a minor by their family, where despite past abuse, the victims craved reunification with their families of origin.

Another family related factor from the background of victims interviewed for this study was the occurrence that they had lost a parent or caretaker at various moments, some in a violent manner, prior to the trafficking experience. Of the 9 trafficking victims and 2 at risk of trafficking individuals interviewed for this study, 3 had a mother who was murdered by another family member, 3 had a mother who had died through natural causes and one person only spoke of her father when talking about family. Of course, no conclusions can be drawn as to whether or not this could have been a factor that may have precipitated the trafficking experience in some way. If family relations were good in the past, the family becomes one of the crucial factors contributing toward the (re) integration process of the victim at present. It is in these cases that return to the family environment may be one of the most significant factors in bringing about not only successful reintegration, but also a sustainable one in the long run. One of the victims interviewed for this study was precisely such a case. As emphasised by her case worker: 
"That is the family of origin that is very strong. So they welcomed her. In difference with other families, they did not accept, they wanted to accept her (...) we saw that she took lots of dependence and she now became dependent in our shelter and sometimes she got out a lot, maybe we saw it even as a risk to stay for more time in X [town in Albania omitted] because of...she could get in contact with somebody...That is why we said ok, now is the right time. She was ready psychologically because, you know, she has passed every period and every stage in a normal way and that is why...we saw that the family is ready so we decided now is time. "(Service provider, Albania, 29 November 2013)

In conclusion, there are various factors from the background of the individual that may influence the (re) integration process in a positive or negative way. Those factors stem from belonging to a vulnerable community, different paths of migration, employment and education history, and most importantly family background.

\subsubsection{Trafficking experience}

The way that the trafficking experience occurred, who the trafficker was, as well as the severity of the experience, has a significant influence on the (re) integration process. The duration of the trafficking experience has not been included as an influencing factor. Firstly, duration was not clear for all victims of trafficking. In addition, taking into consideration the overall context of the trafficking experience, it was found that severity took precedence over the duration.

It has been found through this research that there are instances when the trafficker has been a family member or an in-law. The exploiter of one of the interviewees, who was trafficked while in the later part of her pregnancy, was her mother-in-law. As she noted:

“And there she was talking with one man, Turkish, I don't know him, I don't understand, when I was there, I did not understand what she talked with him. I just understand, she said to him, she can have contact sexual with you for money. My mother in law, yeah. I said to her, I cannot do that, I am pregnant, I don't go. And later, she said for me, you have to do that, because we don't have choice." (Trafficked person, Netherlands, 13 August 2015)

There are many different factors that may damage the trust trafficked women have in other people. However, it should be noted that in the case where the 
trafficker was a person that has in some way been close to the victim, then the trust issues they may have become increasingly severe. Since building trust is an essential element of creating long lasting healthy relationships, cases where the trust of the individual has been betrayed by someone close to them may then take more time and effort in order to recover and (re) integrate.

The nature and severity of the trafficking experience as well as how it occurred may also have a significant impact on the (re) integration process of victims. Based on this research, three distinct types of sex trafficking can be identified.

The first group of victims are those that were either misled by and forced into sex work by a 'loverboy', or were migrating to find work and in the process were forced into sex work. Some of the women in this group were subject to rape, drugged and beaten in the attempt to force them to become sex workers. The traffickers would have total control taking away any documentation and offering no financial compensation for the work done. No contacts outside of the traffickers and clients were allowed. They would have daily money quotas that they would have to reach and would be subject to extreme mental and physical violence and abuse. In the 2 cases where the women had a child, in both cases an infant, they were threatened that the child would be taken away unless they complied with the wishes of the traffickers. At least 2 of the women interviewed that would belong to this group had visible physical marks that they were still recovering from, in one case a permanent injury that was evident 10 years after the trafficking experience. One of the interviewees described her experience:

"Then, he starts to really pressure me [to perform sex work]. To try to influence me. I said, this will simply not happen. I said, then I will go home. I will pack up and go. And that is when everything started. He said, you are not going anywhere. (...) That is how it all started. We started to fight, he started to beat me, more and more often. Every single day. He was hitting me with things, with wood...with a chair. I didn't have a white spot on my body. Everything was blue. Everything was blue. The head. Every single day. I said, no, no, no, I don't want. Every single day, he was beating me. With parts of tables, he would hit me. Four times, I collapsed. (...) He locked me up in a room with just a closet, and nothing else. He said, scream as much as you like, nobody will hear you. Every single day, for 25 days. 25 days, I could not move, go anywhere. The doors were locked. My ribs were broken, 5 ribs broken. My head was full of bumps. Four times I fell in the bathtub. (...) One 
day, simply...it hurt me when I was breathing. When he sees me throwing up, he comes again, he beats me again. He wanted to cut my hair. I have very long hair, he wanted to shave my hair. Horrible things I lived through. When he would close me up in the bathroom, it was horror. It was horror."'(Trafficked person, Bulgaria, 15 October 2015)

Another of the victims interviewed noted:

"They asked me to go, I said, ok, I thought, ok, they are good people. When money is involved, they are other people. Then, I got to know them, and I said, I would not be here. I realised what is happening. Then, after all of that, (...) I had blue marks, everywhere. The colleagues say [service providers], its impossible, how you survived this. (...) I could not be quiet though, I said what happened there. My hands were shaking from fear. They threatened that they would take my baby, and nothing will be left of me."(Trafficked person, Bosnia Herzegovina, 1 October 2015)

The women who have been victims of sex trafficking with the severity described above would not only take more time to recover and (re) integrate, but would also need more types of services. Primarily, it should be noted that the women needed extensive medical assistance as well as psychological assistance. One of the victims interviewed for this study who would be classified in this group has not accepted any of the services offered by institutions so far, as she has had strong family support and two children who she said had given her the strength and energy to go on. However, she reported being extremely nervous, regularly having nightmares, and that she had increased her smoking. As the trafficking experience was recent in this case, it cannot be said if family support was sufficient to help her recover and (re) integrate.

The second group of victims are persons who knew that they would be working in sex work or were somehow persuaded to work in sex work. However, they were thereafter either misled about the working conditions or were taken advantage of and exploited financially, as well as having had their freedoms limited. Some of the victims in this group noted that they were fully aware that they would be working in the sex industry. However, one victim pointed out how she explicitly asked repeatedly to use a condom but was either not permitted or was manipulated and persuaded into not using one. The behaviour of the trafficker towards these women may have been verbally, but usually not physically, aggressive. Victims report hearing threats against 
their family or threats that they will be exposed to their family in order to convince them to continue working. Their freedom of movement was limited and often constrained by the threats that they had received in case they try to leave the trafficker. Some of the victims received some financial compensation, however noted that it was much less than expected, and that a large share of the total fee from the client went to the trafficker. Although victims who have been classified in this group did not report explicit physical violence against them, one victim reported that she had various medical problems, including potentially suffering from sexually transmitted diseases. However, she was never given the opportunity to receive any kind of treatment until she exited the situation. As she explained:

"Yes. I have sterility. I have sterility. Was very not good. Aw, aw, aw, when I have contact. Much client not have sex. When see, aw, aw, I do so. Much client, masturbated, alone. Because he is thinking, what has happened. One man want call hospital. Because I have sex with the man, and come blood. She say to me, $X$ [name of the trafficker omitted], no, it's nothing maybe its menstruation. (...) Now, you see how I feel. Yes, I see the doctor in Romania. Here now. This week will see me, [unclear word] for the doctor. And I wait for the results. But you think what I feel, if one time, one client want call the hospital. Because he sees blood. Because I did one time, Aaaaahhhh! So. I try save me, and what is possible I do, but it's not possible, because was come every time client. Not with everybody you can do, aaaa only with massage, only blow job, or. Not with everybody. And I try, what I can do, I do. But it's not possible with everybody. I was bad, but, I have every time, I have pill from Romania. I call my sister, my sister bring me pill."(Trafficked person, Netherlands, 13 August 2015)

Women that would be classified in this group of victims of trafficking, as with the first group, would need urgent medical attention, as well as additional (re) integration services such as counselling. One of the victims, who had recently testified against the trafficker in her case also reported being extremely worried about her safety, although the trafficker was convicted and was serving a 6 year sentence. Another one of the victims reported that she would not go back to sex work, because she is certain the traffickers would find her again among other reasons. Apart from the immediate medical concerns, women in this group had security concerns and concerns about their economic survival in the future. 
Finally, the third group of trafficking victims are those that took some time in identifying and seeing themselves as trafficking victims. From those interviewed for this study, only the male victim would be classified in this group. However, service providers spoke extensively of such cases that they encountered throughout their work. Such cases would be persons that have accepted and wanted to do sex work, however, were sharing profits with their pimps. Even though for some of the victims such arrangements were acceptable, they are still considered, legally, as victims of human trafficking. In the words of one of the police officials:

"There is also difference between legal victim and feeling like a victim. (...) So, it's common that they don't feel directly as a victim. It's like building up, it's like water in a bucket, and eventually the water is high enough and will flow over the bucket, and that's the moment the woman is willing to step forward and say, hey, this is not right, I feel like I'm a victim. But until that period, it's difficult, she has to in that moment, she is still under the influence of the pimps or under the influence of the trafficker."(Police official, Netherlands, 4 November 2013)

Another service provider noted the following in describing persons that would belong to this last group of trafficking victims:

"Especially when you look at the situation of women from the Balkans, quite a few of them already worked in prostitution in their country and they say 'well, it is just a deal. He takes half of the money.' That is just a business arrangement they made. So then...I have seen them also working in Budapest, in the middle of winter, I was there in January and it was really cold and I saw them working in the street with skirts and having sex in cars. Well, if they are then transferred in this country and they have room where is warm and also they have, in their country, they were used to give away their money so that is not any different, so for them, there has been an improvement to come here. And then we really need to explain 'well, this is not a business arrangement, this is cold exploitation'." (Service provider, Netherlands, 14 March 2014)

As some of the victims that would belong to this group would need to realise that they have been victims of trafficking, their (re) integration process would differ from that of the other two groups, in that the focus would then be on counselling, and thereafter the possibilities of financial compensation to right the material exploitation they were subjected to during the trafficking 
experience. What may be crucial in these situations is to make sure that individuals are protected from recurring exploitation, especially if they consider going back to sex work. In these cases, legislation that exists in regard to sex work and the protection sex workers have under the law, may be extremely relevant. For the male trafficking victim who would belong to this group of victims, the biggest challenge in the (re) integration process seemed to have been accepting that testimony against the traffickers was the right thing to do. He noted the following:

"The work with the psychologist helped me a lot to get positive things from everything. Because maybe you agree with me, but if you have this situation with 4 different people, when everything is finished, and went out, someone thinks, oh my god, was this good or not? Did I make a mistake? Or I think it was not good to come here...for me was like, I share experience, I talk about things people don't talk about. I give you good information, for me is positive, I know I am helping in some way. I know that if I am not telling you that, maybe you will not find that on internet. This is not a recipe for soup or cake. This is not what people share, and its, maybe it makes a little bit angry, people share what is not necessary and don't share the important things. "(Trafficked person, Bulgaria, 14 October 2015)

To conclude, the influence of the trafficking experience itself is significant on the nature and the manner in which the (re) integration proceeds, as well as on the longevity of the (re) integration process. Depending on who the traffickers were, as well as the severity of the experience, the needs of the victims may change, and thus, the (re) integration process may proceed along different paths and timelines.

\subsubsection{After the trafficking experience: Institutions, NGOs, service providers and their role}

Another group of factors that influence the (re) integration process, come from the interactions between the victim and official institutions, NGOs, and other service providers that they come in contact with. In this regard, how identification is conducted, the initial exchanges with service providers, the experience of victims in the shelter, as well as how the contact dynamics with service providers change with time, are all relevant topics that will be explored in greater detail in the next part of this chapter. By looking at the longer-term contact dynamics between victims and service providers, the life of the trafficked persons several years after the experience will also be discussed in order to be able to get an insight into the progress of their (re) 
integration. In this context, experience from victims interviewed with some years of distance from the trafficking event will be taken into consideration as well.

Service providers noted that the majority of identifications of persons as trafficking victims are conducted by the police. However, in addition to the police, women have also been identified by government social service officials, NGOs, SOS hotline calls, doctors, clients, and self-identification. What was emphasised was that services should be offered to victims, regardless of whether or not women would agree to participate in the criminal proceedings against the trafficker. Of course, some victims choose not to take advantage of these possibilities, in particular in cases when they wanted to return to their families immediately, as was the case with one of the trafficked persons interviewed for this study. It should be noted however, that the victim in question had strong family support, but was still suffering from nervousness, nightmares, and had increased her consumption of cigarettes.

Upon identification, it is the first impressions the victim has of the service provider that is important:

"Sometimes, the victims, they say the first contact with people from those institutions is very important. And they say, their impression depends, a lot depends on what the initial contact is. Depending on what the contact is, you may ask for help. But, if the person starts to abuse you, or put you in a position of being blamed, you don't achieve anything. The person feels they are to blame, that they caused what happened to them. "(Service provider, Bulgaria, 16 October 2015)

Consequently, the way the initial interviews and conversations with victims are conducted will have a significant impact on the trust the victim begins to build towards institutional figures. Thus, interviewees noted it is important to give information to the victim in regard to their rights and options immediately, in order to be transparent and reference all the services that they would have access to. Service providers from NGOs and shelters that accept the victims and offer them services or shelter, in particular, noted that often they require very little information from the victim herself and do not 'dig deeper' than what the trafficked person chooses to share.

While some victims like to talk to the service providers, others are quiet in their initial contact: 
"In any case, we do not ask questions about the situation of trafficking at all, not at any moment. We don't think that there is need for this from our side. (...) First, you have, it's called the victim that is quiet. And you have victims that talk. Each person has their own dynamic. They don't have strength. They don't have strength to talk, some of them. There are those that talk, but completely negate what happened."(Service provider, Serbia, 2 November 2015)

The immediate needs of the victim that are usually addressed are physical, such as offering food, clothing, medical assistance, legal assistance, and if needed, shelter. Service providers report that what victims need is a period of stabilisation in a place and environment that is warm. A risk assessment for their safety is conducted, as fear is one of the most common emotions expressed by victims upon identification. In addition, victims are described as being anxious, mistrustful, stressed, depressed, with an irregular biorhythm, low self-esteem, and no real plan for the future. At this initial phase, service providers report that the victims:

"Need space to come to themselves, they are in an acute state, when they are still under the initial state, of going out of the trafficking situation. So, we leave them a bit of space, so that they deal with themselves, their own thoughts, their own feelings."'(Service provider, Serbia, 2 November 2015)

Next, there are several aspects of the accommodation experience and experience with shelters that victims have immediately after identification, which then may shape their (re) integration process. It should be noted that shelter practices are not uniform.

In most cases, victims have a choice as to whether or not they would like to remain at a shelter or acquire their own accommodation upon identification. However, some service providers did mention that, in certain cases, the police were bringing the women to the shelter by force:

"Since the first moment there have been plenty of cases where these girls were taken by force from the police there, and brought here. They were unclear of what was happening. "(Service provider, Albania, 25 November 2013)

Still, it should be stressed that this report was the exception rather than the rule based on the information from those interviewed for this study. Service 
providers usually reported that it was important that the victims chose to stay at the shelter themselves:

"because the only way to ensure the safety of the house is that she also voluntarily comes to the house." (Service provider, Serbia, 29 October 2014)

One of the victims interviewed commented that she simply did not see any other choice for herself but to be at the shelter:

"I don't know. For me is good. Other persons don't like, but for me is good. Because I'm looking at my past, I look in my situation, I don't have choice. You know, somebody tell me if I come here, him help, she help, I have one house, I have one future for my child. But for me is good, I am safe here, I don't know. Yeah. For me here is good." (Trafficked person, Netherlands, 13 August, 2015)

Another difference in practice, was that some shelters had personnel that would remain at the location at all times, some going even further in offering in-house medical services. On the other hand, at other shelters women lived at the location on their own and were simply informed of the house rules, which they were asked to respect. Some shelter rules permitted communication with persons from the outside, however, other guidelines noted that mobile phones should be surrendered. Nonetheless, at all shelters, participating in sex work was not allowed and was one of the strict conditions for staying at the shelter.

Shelters also differed from one another in regard to the group of victims they tended to. In some shelters, women victims of trafficking were placed together with women that were victims of domestic violence. Shelter staff reported that they believe such models work well. However, victims that were placed in such environments reported negative experiences, noting that they felt discriminated against by the victims of domestic violence. In the words of one of the trafficked persons interviewed for this study:

"There are foreigners there and national ones and the prejudices among each other are too much. Sometimes even developed from the social workers and the staff. You see, they see with another eye. They see victims of domestic violence, ok, they see this softness, victims of trafficking they see in another way. And the same happened either even maybe not in a big amount, in X [city in Albania omitted], but in X [town in Albania omitted] as well. Not from 
the staff, because our services mixed, but from the beneficiaries to each other.' (Trafficked person, Albania, 29 November 2013)

Finally, shelter rules showed variety regarding the recommendations given to victims concerning sharing their past with one another. It was more common to find shelters where the staff recommended exchanging experiences, guiding one another, group therapy sessions, peer support:

"We notice that they share. They share with each other, the clients at the shelter. But more often, the good practices, how they dealt with a certain situation, we have a support group, where they tell how they dealt with a certain situation. It's very hard to talk about the survival from the violence."(Service provider, Bulgaria, 16 October 2015)

"What we saw gives the best results, is the peer to peer. We empowered some of the girls that are here for many years. And that has managed to in some way close the traumatic experience, and they have managed to live in a functional way, make some dreams and wishes come true, to be happy the way they want. So, we took some of those ladies, and from time to time, they expressed the need to help others that are in a similar situation. We have people that have passed through similar or same experience, so, from their position, they approach persons that have just entered the program. When they come to the shelter, they can say, I understand, and I am here for you, you can count on me, from today, that I can help you in everything. We think that this is a very equal relationship. Because all the relationships that we build, are still relationships of power, we are not equal. When we talk every day, we realise this. "(Service provider, Serbia, 2 November 2015)

However, not all shelter rules supported exchanges between beneficiaries. Namely, one victim noted:

"No, here it's banned to talk about that. There are problems later, if you do this. I don't talk about that. Sometimes, by mistake."(Trafficked person, Bosnia Herzegovina, 1 October 2015)

A psychologist working at the shelter clarified the rule:

"We told them that the intimate things, they should not share with each other. Because there is a lot of turn over from the safe house. So, when someone exits, they would have all the information of the person. Sometimes they joke 
around, with the kids, in front of the kids, so we want to avoid this. They mostly know what the situation of the others are, but we avoid explicit talking." (Trafficked person, Bosnia Herzegovina, 1 October 2015)

In any case, conflict among beneficiaries while at the shelter was also reported:

"Conflicts happen. Someone doesn't clean the dishes, the glasses. But a small thing is then enlarged, becomes something big, something personal. We are all people, we should be tolerant. Some of them manage quickly, others no. For others it's hard to learn to live with other people."(Service provider, Bulgaria, 16 October 2015)

However, one practice uniform in all shelters that were the focus of this study, is that staff saw it important to organise various kinds of social activities for beneficiaries in which they can participate once the initial period of stabilisation has passed. Even further, shelter staff pointed out that it is extremely important, once the victim becomes acclimated to the environment, to have a structured schedule, things to do, and tasks outside as well as inside the shelter, where possible. Crucially, it was seen as counterproductive to be inactive:

"What my thing is, or what I think we really do good, is that I think we have a really strong or really complete daily activity programme. We have the vision that laying in your bed, and not feel good, is not helping you. You have to get up, you have to get up in the morning, you have to get out, to see people, have contact with people. (...) So, we have training, self defence, biking lessons, cooking, making soup. All kinds of stuff. And I think it helps on many levels, because you get a distraction."(Service provider, Netherlands, 28 March 2014)

It was also expressed that victims often want to be noticed and may crave attention. Thus, in situations where they complete even small daily errands and tasks, it was found important to give positive reinforcement, encouragement, and compliments.

Overall, taking into consideration the services offered to victims and the options for help they have, whether they stay in a shelter or they have their own accommodation, what seems to be of crucial importance is establishing a relationship of trust with a service provider, be it a social worker, 
psychologist or shelter staff. If established, this relationship carries importance not just in the initial stages of (re) integration, but also later on in life. It may be years after the trafficking experience, that service providers report re-establishing communication with victims, at the initiative of the beneficiary. This re-establishment of contact is important not only for monitoring purposes, but also because victims may ask for help with possible current problems they face, and thus reoccurrence of abuse can be prevented, a point elaborated on in greater detail later in this chapter. In establishing a relationship with victims, service providers have described it as an interaction which is not just a consequence of a human trafficking experience, but it becomes a part of life.

The establishment of trust and the initial positive first impressions victims get from service providers are relevant even for those victims that have not yet sought any professional assistance. A victim interviewed for this study, who had reported returning to her family immediately after the trafficking experience but was at the moment experiencing financial and psychological challenges, noted that she had a good first impression from the service provider that conducted the intake upon her return in the country of origin. She stated that she may get in touch with this person, precisely because she felt she could have trust in her, in order to inquire about the possibilities of financial compensation, as well as possible therapy.

Following the initial periods of stabilisation, again, regardless if victims were staying at a shelter, or had their own accommodation, two significant turning points can be identified, which may be taken as signs that the victim is making a leap forward and is ready for the next phase of the (re) integration process. The first is when a victim begins to think more about the future and less about the past. As noted by one of the psychologist interviewed for this study:

"So, at the moment when we passed to the group sessions, it is noticed, that they are psycho-emotionally more stable, and they think more about the future, than about the past. (...) After the beneficiary has passed a 3 months period in the shelter, and conducted these individual sessions, so you can understand that her psycho-emotional situation is not unstable as before, so it is more stable now, now she tells her story as being a third person, and not as she has been the one that has had this experience. And her concern now is closely related only to the future."(Service provider, Albania, 23 October 2015) 
It is usually at this moment that possible employment options are discussed with the victim. It is investigated if the woman would like to continue her education or not, what kind of profession she would like, as well as the training she can possibly attend. Again, this would be the case for women that are staying in shelters and for those that are on their own. It should be noted, that in many instances where a woman could not find employment, she was hired to do some tasks such as cooking at the shelter itself. However, this was not seen as an ideal situation by the women in particular, as it was never certain how long the shelter would continue to exist.

The second turning point and important leap of progress in the process of (re) integration, is the conscious decision to leave the shelter and look for their own, independent accommodation, and obviously, this step is of particular relevance to women that were staying at the shelter following their identification as trafficking victims. However, it is reported that this decision is also accompanied with fears. Fears not only about the economic struggles they may face, but, also in regard to how society, as well as their own families, would accept them. In certain cases, fears of the trafficker can also be present. In this regard, the support of institutions is again important, as organisations were found to offer payments of rent in certain instances, until the woman finds a job, as well as give small business loans for small start-up enterprises.

Throughout this research, one case was encountered where a woman did not make her own decision to leave the shelter, but to the contrary had become more and more dependent on shelter staff. In this situation, it was precisely this occurrence that prompted service providers to make the decision, in cooperation with the family of origin of the woman, to return the woman to her home. This case which was observed 10 years after the trafficking experience had occurred, was one of a successful and sustainable (re) integration process, primarily due to support from the family of origin, even though clear dependency issues were identified.

From this second turning point of realisation of the need for greater independence onward, once again, the relationship built between victim and service provider becomes relevant and significant. Namely, while service providers did report that some victims simply cut contact and did not want to be followed, thus no follow up to monitor their progress was conducted, others did reach out with a shift in intensity of contact, as time went by. Service providers reported that women continued contact and communication with them throughout the criminal proceedings against the trafficker, wanting 
to be informed on the progress with the case or in situations where they were asked to give further testimonies. Some women wanted financial assistance or business start-up funds, so again, would contact their service providers. Others simply needed ongoing assistance with orienting themselves through the system, either in regard to jobs, attaining legal personal documents, social assistance or other official tasks. Others still would simply need someone to talk to, to receive advice, encouragement, or simply confirmation and affirmation of what they are doing. Some would need a check on the decisions they were making, or simply needed to share their everyday problems and dilemmas. Service providers noted that in particular, mothers with children would reach out once they gained greater economic independence and ask for assistance in caring for their children:

"If they have children, they might have problems with children. For the payment of kindergarten, of school. For school material, for clothing of children, for food, for meat, for food. And to be sincere, for a mother it is very difficult to bring them up, only on the generation of income that she has, from the one job that she has. And even in the case when they might have 2 jobs, then there is the problem, where to leave the children. They should get a person to care about the children, when they are absent."(Service provider, Albania, 23 October 2015)

Overall, what is of particular significance is that women, even years after the trafficking experience, reached out to service providers that they trusted, in cases when they were either facing problems within their newly formed families or had fallen into abusive relationships with a partner. For example, a service provider described a situation of a woman that was clearly considered a success case; she had been a part of the (re) integration programme of the institution, stayed at the shelter, found a job, began to live independently, and eventually found a partner and married. However, her mother-in-law and sister-in-law had found out about her past and began putting pressure on her husband to abandon her. It is at this point that she reached out to her case worker once again and asked that they help her out:

"And she calls me one night, crying, saying to me, that I need to meet you. I go and meet her, and she was close to my [place?] she was standing there, saying I am [unclear] and she tells me that my sister-in-law is obliging my husband to get divorced from me. And they will come into the center, and they will ask about me. But please notify the executive director, the former executive director of $X$ [name of assistance organisation omitted], Ms. X 
[name of former director omitted], not to tell about me. I calmed her down, and then she left. I called the executive director and told her about this thing. And after some days her sister-in-law together with her mother-in-law, come here in the center. And they have been received by the executive director, and the executive director told to them that we do not remember this girl at all, it has not been our case. That's why even the prejudice exists in the city. Now, she has even another child, and lives happily with her husband. We say hello to one another. She has two wonderful children."(Service provider, Albania, 23 October 2015)

In some reported instances, women simply asked for advice on their romantic relationships:

"During the time she was here in X [town in Albania omitted], she made the acquaintance of a guy, here in X [town in Albania omitted], but she was afraid to decide about her life. She talked even with us. And I had a coffee with the girl and the guy she had met. Because we were afraid that he might be a trafficker too. But he was a good boy. And to say the truth, I intimidated a bit this guy, because I told to him, that you must be very careful, because this girl is monitored even by police. So, that he was very careful with her. But, that guy, he was very interested in her, and he knew that she was accommodated in the shelter, and he knew that there were girls, even trafficked girls, but he loved her. And when she went to X [city in Albania omitted], he went and met her there, met her family. They got engaged and married, and now they live in X [town in Albania omitted]."(Service provider, Albania, 23 October 2015)

However, in other examples, victims reached out to their service providers in situations where they were once again being exploited or in a violent relationship:

"So, depending on the problem, it has happened that the victim of trafficking, our clients, through the telephone inform us of the current violence they are experiencing from their partners. So, this has nothing to do with human trafficking. There is violence in the family, so the contact with us still helps. We put them in touch with organisations that are dealing with this problem. We also report it. So, we were also in urgent situation, when the violence was happening. So, we had to call the police, and were intervening, we had to inform the center for social work etc. So, it really depends on the client."(Service provider, Serbia, 30 October 2014) 
In conclusion, what seems to be crucial over time as far as the institutional assistance programs for victims are concerned, is that the relationship built with service providers is one based on trust, so that the woman may feel confident enough to reach out in situations of difficulty, but in particular in situations of recurring violence and exploitation.

\subsubsection{After the trafficking experience: Economic factors}

Factors related to the present-day situation of the victim in regard to education, employment, and accommodation also have a significant influence on the (re) integration process. Not having any financial means for survival puts women at risk of being exploited once again.

As it has been already noted some victims of trafficking have only rudimentary education, which one could argue limits their employment options. However, based on the interviews conducted as part of this study, it should be noted that service providers expressed doubt as to the importance of education in these cases. As one interviewee acknowledged:

"We had a client that we all thought that it's best to go to school. But after the trauma she lived through, she said, it's not even on my mind to go back to school. So, you say to yourself, ok, I will try to understand this decision, I understand. So, when the cases last many years, 3, 5, you think, ok, I will convince her on the importance of education during this time. But how to do that, in a country where you know that even people who have gone to university, have studied, they still may not have a job, or a person with no education, for whatever reason, may manage to get a job before someone that is educated. So, how do you make a case for education?"(Service provider, Serbia, 2 November 2015)

One of the interviewees who was a victim of trafficking, noted that although she had higher education and had finished university, it was very difficult to find a job based on her educational background. However, what is of particular interest is that although victims may be doubtful in regard to the practical usefulness of their own education, those that had children certainly wanted them to have the opportunity to receive higher education and stated that they would do everything in their power so that their children have a chance to go to university.

Regarding employment, some victims explicitly expressed that the type of job they have does not matter, that they would do any job that gives them some 
kind of financial security. However, service providers often pointed out that some victims become disappointed by the low financial benefits from jobs they find after trafficking and would rather go back to sex work due to the greater economic returns:

"There is this stigma about money. The money they used to have, to be able to afford their life. So, now, when they find a job, for 150 dollars a month, they feel offended, they say, what, I used to have 150 dollars for two days. They are used to a different type of life. They forget they are in Albania, and there is a different way of life here. "(Service provider, Albania, 25 November 2013)

Some women, through the trainings offered to them by shelters or NGOs, could specialise in a skill that they could then use to find a job. One example that came up often throughout this research was cooking lessons. One of the victims interviewed, whose ideal job was to be a cook, received appropriate training but could not find a job. In this case, she was hired by the shelter to prepare food for the beneficiaries. Of course, as already mentioned, the fear in these situations is that the job is there for as long as the shelter is there, and that is dependent on acquiring external grants and funding.

Finally, it can be said that although employment was mentioned as one of the most important components that lead to successful and sustainable (re) integration, it should be noted that if unemployed, the (re) integration process should certainly not be seen as a failure, but that it just makes the women potentially more vulnerable to exploitative situations in the future. Victims interviewed as part of this research who were at the moment unemployed, were not in a situation of violence or exploitation, due to a variety of different factors, such as family and institutional assistance.

In conclusion, the influence of education on the (re) integration process would need further study and exploration, however, having employment certainly has a positive impact. Still, unemployment should not be seen as a reason to mark a (re) integration process as a failure.

\subsubsection{Personal characteristics, challenges, motivations and coping}

There are some personal characteristics of the trafficked person, which may contribute toward a successful (re) integration process. Service providers noted that being smart, having the ability to think, being emotionally developed, open, honest, persistent, consistent, principled, and stubborn were 
characteristics of women that have been successful in their (re) integration process. It was commented that women should also have the will to change their quality of life, take advantage of the help offered, and fight to continue forward.

However, based on the interviews with women for whom the trafficking experience was recent and the reports from service providers, most women following the trafficking experience report feeling dirty, used, not human, nervous, ugly, lonely, disrespected, and as if they deserved what happened to them:

"It had to happen, something had to happen to me in life, so that I can value, and examine the little things I have. Because until something happens, people don't realise about what they have in their life, the little things. Something very bad had to happen, I paid a very high price. But, the higher the price, the more you value the little things, you accept the little things, that you have in life."(Trafficked person, Bulgaria, 15 October 2015)

Women also reported feeling fear for the well-being of their children, fear of the trafficker, from exposure, as well as from the possibility of the same exploitation happening again.

Thus, what are some of the ways in which the personal characteristics of success can be reinforced, rather than the negative feelings women experience, in particular the fears and the self - blame after the trafficking experience? One of the primary motivating factors mentioned, which will be discussed in greater detail in the next section, is family and children. Moreover, women noted that having a job was also of help, as was counselling with professional therapists. Service providers noted that the primary goal of therapy is to make sure that the victim no longer blames herself for what happened, increase self-esteem, or simply release. Another important issue for victims of sex trafficking is loss of trust. Victims interviewed for this study stated that they either trusted nobody, or only trusted a small intimate circle of family and friends. Thus, trust is another issue addressed in therapy, and overcoming trust issues may have a positive influence on the (re) integration process.

Service providers observed that working through the trafficking experience itself with a professional therapist is also important, in particular so that after such therapy sessions, the women can close that chapter of their life and not 
think about it any longer, as far as that is possible. This is in fact how one of the women interviewed for this study, with the longest time distance from the trafficking experience, looked upon it today. Others noted that they were either too busy to think about it, tried to keep busy, so as not to think about it, or fought their urge to think about it. One woman noted, that remembering what had happened, is always extremely difficult:

"I don't want to think about the past. Sometimes it comes in my dreams, and I want to strangle myself. Sometimes, I wanted to strangle my own son. But I hope to god he is good and healthy, and I hope I remain healthy too. Now, I am ok, thank god."(Trafficked person, Bosnia Herzegovina, 1 October 2015)

In conclusion, women may have some inherent personal characteristics that determine if they manage to recover and (re) integrate successfully. However, following the trafficking experience, and for quite some time afterwards, many women may have a negative self-image. The presence of such feelings should not be considered a determining factor of success or failure of the (re) integration process, as a negative self-image can be improved by means of cognitive-behavioural therapy. Finally, women may have difficulties in fully overcoming what has happened to them, thus, it is important that once they work through the experience and realise they are not to blame, they should not be reminded of the experience nor asked to talk about it against their will.

\subsubsection{Social support: family and friends}

The influence of the family of origin on the (re) integration process of women has already been discussed in the section covering the background of the woman. However, in addition to the family of origin, what seems to have a significant influence on the (re) integration process are also the families that are formed by the victim, whether prior to the trafficking experience or newly formed families after the trafficking experience. All women who reported having children stated that they are one of the primary motivating factors for them to do well. Women that were currently separated from their children, as they were recovering in the country of trafficking, voiced their wish to be reunited. Those that were currently with their children but residing in an institutional setting mentioned that being with their child is what brings them happiness.

Furthermore, what received particular emphasis as a significant stabilising factor for trafficking victims, was finding a partner, getting married, and forming their own family based on healthy relations. 
"Then, she got married, she has a child. So, everything ended in a very good way."(Service provider, Bulgaria, 13 October 2015)

"Many of them we consider them as successful cases the ones that have created their own families. (...)I would define it if she has created a family, a sound family, and if her past it does not constitute a problem for her husband and for the relatives of her husband."(Service provider, Albania, 23 October 2015)

"Ideally, and most successful (re) integration, when the person that has survived an experience, they enter into the normal flow of life, they find a job, form a family, they have their most intimate family surrounding, a partner, children, and they live some kind of a normal life.(...)those cases with foreign victims, girls, that have married. And here, that is the best (re) integration.' (Police official, Serbia, 30 October 2014)

Finally, one of the trafficked persons interviewed for this study expressed the following wish for the future:

"Maybe I married with somebody who like my boy [her son]. Maybe. If I meet somebody good, and he don't make problem because I have boy. Maybe the man can have one boy, one girl. I don't want alone forever. (...)Because I don't want to speak with nobody. My sister not like, if I say, I have problem. Me, alone. Everybody is married. My sister married, my brother married, only me. Because maybe I am ugly, I don't know. (...) And little by little, when I work and have my little house, I don't know where, maybe I look for somebody friend, relation or maybe more. If, if its possible. If. Maybe married. Why not? Yeah, I think, maybe its possible. I cannot marry with everybody, only because I am alone. I want easy man. Because I was very very very ugly life [had a very ugly life]. All my life."(Trafficked person, Netherlands, 13 August 2015)

In regard to the role of friends in the (re) integration process, women reported that they either had no friends or very few friends, and while some admitted to not being social at all, the one case where she reported that she was social, still perceived that she did not make good friends:

"I have many acquaintances, no friends. (...) I don't want. I have been burned many times. If someone wants to sit for a coffee, and chat, ok. But I don't have friends. I never got anything good. I've always given a lot, a lot, never gotten 
anything good in return. Better not to have, than ... acquaintances, you sit for a coffee, then bye bye. So that nobody knows what you are doing, what is really happening in your life. People know a lot, and then they become very jealous, they are invasive. Why should I also create these problems for me. I talk to my mom. With her, with her I talk. Also with the children. My children know everything. I don't hide anything."(Trafficked person, Bulgaria, 15 October 2015)

In conclusion, the role of children and reunification with children and family following a trafficking experience where the members of the family were not involved in the trafficking experience has a significant, positive influence on the (re) integration of women. However, based on information presented by service providers as well as on wishes expressed by victims themselves, what seems to be seen as an ultimate goal of the (re) integration process, and the most significant stabilising factor in the long run, is finding a trustworthy partner, marriage, and the formation of a new family based on healthy relationships.

\subsection{Discussion}

A variety of factors that influence the (re) integration process were explored in this chapter: (1) background of the individual, (2) trafficking experience who the trafficker was and its severity, (3) the role of institutions, NGOs, and service providers (3) economic factors (4) the personal characteristics, challenges, motivations, and coping mechanisms of the victim, and finally (5) social support. Identifying the relevance of economic factors is commonplace in research focusing on (re) integration following a trafficking experience (Surtees, 2010, 2013; Sharma 2015). In regard to social factors, although complex issues of reunification with children have been outlined by other researchers (Brunovskis \& Surtees, 2012), in all cases of this research, children were a positive, motivating factor and the women did not delve deeper into the complexities of these relationships. The reason may be that the questionnaire did not go deeper into this issue. But also that, for some of the victims, the children were still very young and not aware of what the mother was going through. Motivation throughout the (re) integration process (and as already noted in the context of this study, children often being mentioned as one of the primary motivating factors for victims) has also been identified as a relevant factor for (re) integration in other research (Surtees, 2010). However, the importance of the trafficking experience, in particular the exploration of how its longevity versus severity may influence (re) 
integration, which has been discussed in this chapter, has not been dealt with in other work to the best knowledge of the researcher.

The identified factors that influence the (re) integration process can be categorised based on the ecological model of sexual assault recovery (Neville \& Heppner, 1999), at the micro, meso and macro level. Within this model, the meso system consists of factors related to building and re-building social relations (family of origin, newly formed families and friends) as well as institutional factors (support from NGOs, government and other institutions, as well as factors influencing the formation of relationships with service providers). Economic factors mostly belong to the meso level. Some factors that are related to the background of the individual (wish to migrate, background economic situation, educational and family background) belong to the micro level of the ecological environment. All personal characteristics (challenges, motivations, coping) as well as all factors related to the trafficking experience (means of trafficking, the identity of the trafficker, severity of the trafficking experience) belong to the micro level of the ecological environment. However, as with the elements of (re) integration, some influencing factors from the area of economic well being as well as some elements of the background of the individual (belonging to a specific social group) belong to the macro level of the ecological model. The general economic situation of the country in which the survivor resides will determine some aspects of her economic functioning, regardless of her own role within that context.

\subsubsection{The importance of forming healthy social relationships}

Having outlined all of the influencing factors found through the course of this study, a few conclusions can be drawn regarding the relative importance of each. The background of the individual is significant in as much as it means that the victim may or may not have a social support system in place. It may also have an impact on the ability of the person to form healthy relationships in the future. The trafficking experience may have a significant influence on the (re) integration process, particularly in regard to the physical and mental recovery of the victim. And the feelings the victim has toward herself may have a significant influence on the recovery aspects of the (re) integration process. However, all of the issues noted can be dealt with in the initial interventions through health assistance, as well as therapy. Pursuits of the victim that have to do with economic aspects of her life may be positive influencing factors, if they are driven by the autonomous decision making of the victim. However, what seems to underline and supersede all of the above 
are the social factors - relationships built with service providers, relationships rebuilt with existing family members, or relationships built with new families that were established after the trafficking experience. As long as a relationship based on trust is built with service providers, challenges - economic or psychological - that may arise in the future, may be dealt with in a way that keeps the woman out of potentially exploitative situations if she feels she can come to the service provider for help. Along the same lines, if a support system based on healthy relationships is in place in the form of a family (whether the one of origin, one formed before the trafficking experience, or one that is formed following the trafficking experience) most challenges can be dealt with in such a way as to not endanger the victim again.

As already noted in the literature review of this chapter, other scholars have dealt with the role of the family in the (re) integration process of trafficking victims and its potential positive or negative influences (Surtees, 2010, 2013; Brunovskis \& Surtees, 2012). However, what existing research seems to focus on is the different challenges that family reconciliation poses. What comes out of this current research is that when exhibiting positive influence, such as the situations where the family has understanding for what the victim went through or when the victim has the capacity to form a new family, based on healthy relationships, that positive influence seems to be the most significant factor leading to successful and sustainable (re) integration process. Of particular note is the creation of new families after the trafficking experience, an aspect that has not been explored in depth. The significance of these new families can be seen here through the focus of service providers on the importance of marriage. At first sight, it may seem that service providers are promoting for these women a replacement of one situation of dependence, with another. However, the researcher believes this is not how service providers see 'marriage' for trafficking victims. To understand what a new, healthy 'marriage' means in the context of (re) integration of trafficking victims, a reference to literature on the 'need to belong' and selfdetermination theory (SDT) is necessary.

The belongingness hypothesis states that "human beings have a pervasive drive to form and maintain at least a minimum quantity of lasting, positive, and significant interpersonal relationships" (Leary, Mark R. \& Roy F. Baumeister, 1995, p. 497). According to Leary \& Baumeister (1995), the two main conditions of a relationship that must be satisfied for it to fulfil the belongingness need, is that contact between the persons involved is frequent and that the bond itself is stable and based on mutual care. They further 
explored such relationships and came to some conclusions that are of relevance for this study. Namely, such bonds can be created also in adverse conditions, that is, in situations where people live through a crisis together. Once formed, there is an innate resistance to breaking such bonds. Finally, such bonds create positive emotions, while the deprivation from such bonds may bring about pathological consequences, such as mental and physical illnesses (Leary \& Baumeister, 1995; Leary, 2010). All of the above, are just some aspects of the need to belong that position it as a fundamental social motive, which should explain much of human behaviour (Leary \& Cox, 2008).

Having said that, for a relationship to fulfil the belongingness need it must include frequent contact between the two parties and be based on stability and care, the discussion inevitably leads to the issue of marriage. Marriage in this context is seen as a positive turning point, when a bond receives a permanent status (Leary \& Baumeister, 1995). Thus, marriage becomes "an institutional means of satisfying the desire for acceptance by offering a sanctioned way to promote long-term belongingness" (Leary \& Cox, 2008, p.35). Further on, the inclination to marry, even if seen as an attempt to conform to societal expectations, in addition to being a way of solidifying the longevity of a caring bond, may not be seen as a loss of autonomy, but in this case, conformity may be natural and adaptive in the pursuit of acceptance from others, and thus satisfaction of the most basic social need to belong (Leary \& Cox, 2008).

The issue of trust and its importance has been explored by other scholars as well (Walsh, Black \& Koser, 1999; Koser \& Pinkerton, 2002; Brunovskis \& Surtees, 2012). However, previous studies focus more on the trust persons have in the information they receive from service providers, or on the importance of establishing trust between service provider and victim, so that the victim accepts assistance. This research places emphasis on the importance of trust between a beneficiary and the service provider, and the significance of building strong personal relationships because of what that would mean for future (re) integration efforts. The establishment of a more personal relationship based on mutual trust is the key for the (re) integration process and its sustainability in the future. Service providers, in particular case workers and social workers, are among the first to face the victim after identification as a trafficked person. This contact is crucial. If the victim gets the impression that they can trust the service provider, they will ask for help, not only in the initial (re) integration period, but also later on. It may be that 
in the future life the victim builds, she decides not to share her past experiences with her new family. Thus, in this regard, the service provider remains as a contact that knows her entire story, that she may talk to about anything, at any time. Brunovskis \& Surtees (2012) talk about the importance of the 'monitoring' phase of the (re) integration process and how it can be very helpful but is not always feasible that a service provider closely follows the progress the victims make after they exit the phase of services offered by their assistance organisations. However, monitoring becomes unnecessary in certain cases if the woman feels she herself can turn to the service provider in a time of need, rather than being tracked down and observed by the service provider on a regular basis. It has been found that monitoring is in many cases too costly for the service organisation, and in others, the victim does wish to cut ties with these organisations. Thus, establishing a relationship built on trust, where the victim can always refer to her case worker in times of need herself, may make the monitoring phase beyond a few years, unnecessary.

\subsection{Limitations and implications}

Having reviewed the primary findings of this research, some of the limitations should also be discussed. The primary limitation of this research is the small number of victims who have been interviewed and within this group, the lack of examples of women that have been primarily financially exploited and have chosen to return to sex work, following a trafficking experience. However, it should be noted that this group is difficult to access and would require methods that were beyond the scope of this study. In addition, it should be said that service providers are also very knowledgeable and more willing to openly share their experiences (although it should be noted that their views may not reflect those of the women). This study has taken advantage of this knowledge in particular, thus also minimising the negative impact on the victim themselves that may be caused by discussing the trafficking experience. Finally, a strength of this chapter is that it has tried to identify some factors that may influence (re) integration in the long run - a topic that has not been studied thoroughly so far. Trust built between service providers and victims, as an important asset in sustaining the (re) integration process in the long run, is one of those factors.

\subsection{Future directions}

This chapter stemmed from the identified need to look at what factors influence the (re) integration process in practice, the overall dynamics of the 
(re) integration process, and the need to distinguish the more important from the less important factors, influencing (re) integration not just immediately after the trafficking experience, but also several years down the line. Studies focusing on the short-term (re) integration processes identify the importance of trust established between victim and service provider (Burnovskis \& Surtees, 2012). However, what would be useful going further is trying to explore what the relationship built on this trust may imply for the future. The importance of families and the importance of re-building intimate relationships with spouses from before the trafficking experience has been studied (Brunovskis \& Surtees, 2012). However, more focus must be placed on the role of newly established relationships and families formed following a trafficking experience and their importance for the (re) integration process in the long run.

Thus, there are significant policy implications based on the results of this current research. It has been found that one of the primary influencing factors that may have a positive impact on the (re) integration process of victims in the long run, is the trust built between service providers and victims, during their very first interactions. With this in mind, trainings that would teach service providers how to best build this trust from the first moments of interaction would be greatly beneficial. However, what also warrants attention is emphasis on building long lasting relationships between service providers and victims. Such relationships would ensure access to services and help for the victims not just immediately following a trafficking experience, but also several years after, thus lowering the chances of recurring exploitation.

One additional policy implication should be the importance to be placed on building new, healthy relationships after the trafficking experience. It may be that this issue is tackled between victim and therapist, but perhaps there can also be other ways in which to ensure that women have the capacity to establish new and healthy bonds. For instance, the establishment of a relationship built on trust with a service provider as the first person of contact after the trafficking experience may contribute toward building other healthy social relationships in the future.

Finally, based on this research, friendships seem to have a small role in the (re) integration process. The reason could be that victims have persistent trust issues and still face difficulties forming relationships outside of family or outside of the circle of service providers who are aware of their past 
experiences. What does this mean for the (re) integration process in the long run? Is it significant? Or is it the case that this research failed to capture individuals for whom friends may play an important role in their (re) integration? These questions may be reasons for further exploration of the role of friendships in the (re) integration process of trafficked persons. 


\section{Chapter 6: Trafficking of Women for Sexual Exploitation in Europe: Prosecution, trials and their impact $^{14}$}

\subsection{Introduction}

"Often, according to the law it's possible [prosecuting and convicting traffickers], but in practice it's impossible."(Police official, Serbia, 30 October 2014)

The introduction of anti-trafficking legislation at international and national levels has been heralded by academics, politicians, lawmakers, and practitioners alike as a major step forward in the fight against human trafficking. In fact, the definition of human trafficking, as presented in the Protocol to Prevent, Suppress and Punish Trafficking in Persons, Especially Women and Children (from here on the Trafficking Protocol), is by itself a major step forward in the fight against human trafficking. Since 2000, when the Protocol was introduced, legislation from the international level using the definition as a starting point, has in many countries slowly trickled down to the national level and has thus given people on the ground the tools to battle this phenomenon.

However, while an undeniable step forward, it is only a step forward in a much larger battle, and a considerable amount remains to be done. Attempting to evaluate the effectiveness of criminal justice responses to trafficking in the US, Farrell et al. (2014) gathered data from 140 cases of human trafficking in 12 US counties and conducted interviews with individuals from law enforcement, prosecutorial bodies, and service providers. They concluded that failures of state and federal authorities in the US to effectively prosecute trafficking cases is due to 'legal, institutional, and attitudinal challenges' when using anti-trafficking laws (Farrell et al., 2014, p. 161). Spohn (2014) places legislative reforms regarding human trafficking in the same line as reforms that were introduced to improve prosecution and conviction rates in areas of sexual assault and domestic violence cases, but clearly failed, as great

14 An earlier version of this chapter has been published as: Meshkovska, B., Mickovski, N., Bos, AE., \& Siegel, M. Trafficking of women for sexual exploitation in Europe: Prosecution, trials and their impact. (2016). Anti-Trafficking Review, 6(6). doi:10.14197/atr.20121665 
emphasis is placed in all three areas on the testimony of a 'genuine' victim who is beyond any moral reproach (Spohn, 2014, p. 175). Goodey (2004) looks at the prosecution of trafficking cases in the European Union and notes inadequate witness protection programmes as a legislative limitation (Goodey, 2004, p. 6). Goodey (2004) goes further to recommend that traffickers should be prosecuted under legislation other than that specifically introduced for trafficking, for instance, for charges such as money laundering. Such prosecutions will also lessen the burden placed on the victim throughout these processes.

Thus, the questions arise, what are some of the issues that come up when implementing human trafficking legislation in practice? What can we learn from the experience of the professionals in the field and trafficked persons themselves? Finally, and most importantly, what can be done to increase the benefits of such laws for the victims, or at least to make sure that they are not harmed by the implementation? This chapter will answer these questions, in the European context, by focussing on Albania, Bosnia Herzegovina, Bulgaria, Serbia, and the Netherlands. The focus of this chapter is on female trafficking for sexual exploitation as it is one of the most prevalent and severe types of trafficking in the countries mentioned above.

\subsection{Methodology}

For this chapter, a selection of European countries was made because of the progress they have made in introducing anti-trafficking legislation, as well as the prevalence of the issue of trafficking in those same locations. To provide a representative overview, countries from Eastern and Southeastern Europe (Albania, Bosnia Herzegovina, Bulgaria, Serbia) as well as Western Europe (the Netherlands) were selected. The countries chosen for the study are, with the exception of the Netherlands, principally countries of origin of victims. There were several reasons behind this aspect of the selection process. First, it is in countries of origin that victims and service providers are often most available for interview. Although trafficking cases may be identified in countries of destination, the trafficked person often wants to return to the country of origin immediately after identification. Additionally, the trafficker may also return to the country of origin, which may be his/her country of origin as well. Second, prosecutions - not least for internal trafficking - do take place in countries of origin. And third, as noted above, research to date has generally focussed on countries of destination, and thus an overview of the same processes in countries of origin seems to be lacking. 
The main part of this chapter focusses on the issues that arise when speaking to professionals in direct contact with victims, as well as those most intimately affected - the trafficked persons themselves. For this purpose, seven interviews with trafficked persons were conducted. Additionally, 33 interviews were conducted with service providers from the target countries (6 from the Netherlands, 9 from Albania, 5 from Bosnia Herzegovina, 5 from Bulgaria and 8 from Serbia). They include program managers, social workers, psychologists, psychotherapists, police officials, lawyers, directors of antitrafficking NGOs, shelter coordinators, and crisis hotline operators. Data was gathered through semi-structured interviews with follow up probes. Victims were asked if they have participated in the criminal proceedings against their trafficker and what that meant for them. Victims were also asked about their life prior to the trafficking experience. They were asked to talk about the trafficking experience, if they so wished and finally, about their current situation. In regard to their current situation, victim respondents were asked about their economic standing, physical and psychological well-being, as well as their social life. Service providers were asked about their contact with victims, identification, and needs from the initial period of communication until their last communication with victims, as well as what they consider successful and not so successful cases of recovery and (re) integration. Within this process, service providers were asked for their view on the impact of criminal proceedings on the victims. Service providers were also asked about their, and others', attitudes towards sex work, in addition to their personal feelings towards their job. All interviewees were asked if they would like to give additional comments on issues not mentioned or touched upon but related to the topics of discussion throughout the interview. Conversations lasted from 30 minutes to 2 hours and were held at offices, cafes or private homes depending on the wishes of the interviewee. The interview protocols were reviewed and approved by the Ethics Committee of the Faculty of Psychology of Maastricht University. Respondents did not receive any payment for the granted interview.

The analysis of the interviews has brought to the surface certain themes: (1) when referring to length of a process against a trafficker, it has been noted that it can last up to 10 years, (2) secondary victimisation, (3) specialist training and interviewing skills, (4) information and trust, (5) protection from intimidation, (6) not just conviction but financial compensation and finally, (7) the label 'victim' and the wish to testify, all of which are discussed in detail below. These are issues about criminal proceedings that, if addressed appropriately, can contribute towards the better recovery and (re) integration 
of trafficking victims. Trafficking for the purpose of sexual exploitation is among the most severe types of trafficking, it is the form of trafficking that service providers are most familiar with and as such has been chosen as the main focus of this chapter. As trafficking for the purpose of sexual exploitation predominantly affects women and girls, female victims of trafficking for sexual exploitation are the main target group of this research. Although men are also victims of trafficking for sexual exploitation, there could be significant gender-based differences in regard to experiences related to the prosecution of the trafficker. Thus, focus on male victims is outside of the scope of this particular chapter.

\subsection{The practice of the law: The stories of trafficked women and service providers}

Trafficked persons are the primary source of information when it comes to understanding the impact of criminal proceedings and prosecution of traffickers on the identified victims themselves. Additionally, service providers that work with trafficked persons, such as lawyers, social workers, psychologists, psychiatrists, program managers, crisis hotline operators, leaders of anti-trafficking NGOs, shelter coordinators as well as police officials, often have extensive experience and knowledge from which many lessons can be learned. Professionals in the field are also a valuable source of recommendations: of what to do, what not to do, what works, and what does not. Thus, based on the interviews with trafficked women and service providers there are a number of themes that have been identified. Additionally, as the stories of trafficked persons contain many characteristics that are indicative of issues raised by practitioners in the field, each section of identified general themes will begin with the re-telling of the personal experiences of the victims interviewed for this chapter.

\subsubsection{Length of the criminal justice process}

"Those trials were...come and go every time the government changed, the chief of the police was changed... We got really frustrated...10 years following the court...Tension ...The psychological tension was big...Such a psychological pressure. The guy, after 10 years after changes of government and so on, he got the decision of court for 7 years in prison but it was never served." (Trafficked person, Albania, 29 November 2013)

Throughout the interviews with service providers from the target Balkan countries it was often mentioned that criminal proceedings against traffickers 
can last up to 10 years. Various explanations for such drawn-out timeframes were given, such as a change of governments, change of judges, and change of court. In certain cases, so much time has elapsed between the criminal and the civil procedure, through which victims ask for financial compensation, that due to the short sentences imposed on traffickers (recruiters and exploiters, which in certain cases are the same person) the perpetrator is already out of jail and fails to appear for the civil procedure. It should also be noted that during the civil procedure for compensation (a procedure that must be initiated and funded by the victim), the burden of proof is on the victim, not the defendant. And civil proceedings, which effectively require everything to be done over again, may last just as long as the criminal procedure.

Although interviewees from the Netherlands also note that criminal investigations as well as criminal proceedings can last a long time, there is one stark difference between that country and the others studied in terms of the impact of such prolongation on the well-being of the victim. At the end of a trial in the Netherlands that results in conviction, victims will most likely be financially compensated as a part of that process. In addition, foreign victims residing in the Netherlands for the duration of a trial that lasts beyond 5 years are entitled to request Dutch citizenship, and thus are not compelled to return to their country of origin unless they desire to do so.

\subsubsection{Secondary victimisation}

"I was a bit nervous, when they called me to the police, to talk, the first time. Whenever I see there is something from the police, I am afraid. I know that I shouldn't be afraid. I said, I'm afraid, I don't know why, maybe it's my habit..."(Trafficked person, Bosnia Herzegovina, 1 October, 2015)

Giving one detailed statement of the trafficking experience is a severely traumatic event for a trafficking victim. Unfortunately, it often happens that multiple statements must be provided throughout a long time period, each going into great detail.

A social worker from Serbia describes the atmosphere before a victim of trafficking has to testify in court:

"We always try to go with them, to be their support, in case it's necessary, even if the psychologist prepares them for the testimony. Because they often feel a big fear, and are very upset, especially before the testimony, and it's 
important to be there for them, and to explain to them what the trial means, and what it may bring for them. Because of course there have been situations when they wanted to exit from all of that, to retreat, there are also cases when the trial has been postponed, because of the inability of the victim to face, not only the trafficker but to say what happened, in public, because of the bigfear they feel." (Service provider, Serbia, 10 October 2014)

A psychologist, also from Serbia, notes the following about the criminal process:

"This is very traumatic for women, because they have to again and again appear at the trial, to give statements, to meet the perpetrator. And that makes the recovery hard, and it brings back some of the traumatic experiences that she had, while she was trafficked. So, in that period, we have the most intensive communication with the women. After the end of the trial, then slowly, they also put a 'period' at the end of the experience."(Service provider, Serbia, 22 October 2014)

This situation of telling and re-telling the trafficking experience in such detail is particularly troubling considering that one of the ways in which women manage to move forward is by leaving the past behind. That aspect is captured well in the words of another psychologist:

"When they come to the shelter, they say 'I want to change, and not mention again what has happened in the past." (Service provider, Albania, 27 November 2013)

In fact, it is the practice of shelter staff interviewed throughout the countries studied to not ask anything about the past and only work with what the women themselves decide to share. Thus, victim testimonies during trials, in often intimidating and sometimes hostile court environments, can hinder the recovery and (re) integration process of victims. A more friendly environment may be one in which the prosecutor is more familiar with the victim.

Some of the interviewed service providers mentioned the possibility of using video to provide testimony. However, it is also remarked that even when this is available, it does not make a significant difference on the mental well-being of the victim. 
A Serbian police official interviewed for this chapter emphasised that victim testimony was not their primary concern, but victim protection was:

"We identify victims of trafficking independently of the acceptance to participate in criminal proceedings. We don't care about that, we don't care, if a victim is participating in trial, that is relevant to the prosecution. We want to protect the human rights of the victims of trafficking, and it's not important if that person has accepted to testify in criminal proceedings. "(Police official, Serbia, 30 October 2014)

A Dutch police official noted that there are different ways in which an investigation may be started and not all involve an immediate statement from the victim. When the investigation is initiated following a direct complaint by the victim, which may at some point translate into a testimony given in court, the police first check if the person is indeed a victim of human trafficking. If it is a case of human trafficking, the victim is given a 'reflection period' of up to 3 months, in which time she decides if she would definitively want to press charges and testify against the traffickers.

In some instances, progression of the case may not require the victim to testify in court. Rather, the victim is invited to provide a 'witness statement' to the police or the court. According to the Dutch police interviewee, this is often "less [hard] for the victim". However, although the case may be initiated based on a 'witness statement', it may occur as the case progresses that this is not sufficient and that the victim is still requested to give a testimony in court by the judge.

There are also situations in the Netherlands where the investigation is initiated based on an anonymous tip or information that is gathered through another investigation. In that situation, the interviewee stated: "The legal system in Holland makes it possible to control the prostitution business, both legal and illegal. These kind of controls also provide information on which you can start an investigation.”(Police official, Netherlands, 4 November 2013).

Victim testimony must, in any case, be supplemented by additional evidence. Primarily, according to the police official, the statement of the victim is always checked to make sure it is truthful. In cases where victims arrive in the Netherlands by plane, the passenger lists are checked. Internet, social media, and cell phones are also checked for locations mentioned in the statement. Other persons who may be able to confirm the statement or 
investigation information are identified. Finally, as detailed by the police official "we follow the money". Calculations of the victim's costs and benefits are made and double checked against the administration of the brothel keepers. All additional evidence is important because in cases where it is lacking, it often comes down to the statement of the victim against the statement of the suspect.

The Dutch lawyer and the police official quoted above both emphasised that there are cases when the police do not want to ask the victim to testify at all.

According to the police official:

"Sometimes we see a victim who is so mentally unstable that we think her statement will not stand during investigation/trial. Sometimes because she is emotionally harmed but sometimes because of her mental capacities. We also evaluate these questions together with the social workers and psychiatrist if a statement will cause damage to her treatment/recovery. If necessary we will drop the case."(Police official, Netherlands, 4 November 2013)

However, an exception may be made: "if there are more victims harmed or in danger by the same group/suspect...these are hard decisions as you will understand...sometimes it is a tactical decision: if we think a statement will reduce the chances of successful prosecution because a defence lawyer will be likely to cause doubt by a judge during interrogation."(Police official, Netherlands, 4 November 2013)

\subsubsection{Specialist training and interviewing skills}

"They [the police] told me, you didn't do anything bad. These people did something bad, they are bad, don't go with them again. When I really saw what they did, I was sick, I wanted to hang myself, God saved me....I'm not afraid anymore."(Trafficked person, Bosnia Herzegovina, 1 October, 2015)

The initial contact with the victim after identification is crucial. It is in these times of fear and low trust that those in touch with the victim must take special care to assure victims that they are not to be blamed for what happened, that they are the ones whose rights have been violated.

Law enforcement officials interviewed for this study placed great emphasis on the need for specialist education training of police, prosecutors, and judges. A Dutch police official pointed out: 
"You need, to do that part of the job, you need extra certification, extra diploma. And in Holland, this training is 256 hours of study for the detectives, and within this course there are three exams, and one third of all the participants fail the exams. So, we are trying to raise quality in investigation of human trafficking."(Police official, Netherlands, 4 November 2013)

A police official from Serbia noted the following:

"We in Serbia have a specialised police, working with human trafficking, sensitised about human trafficking issues...in 27 prosecutors offices, there are 27 contact points that have gone through the education. They are appointed by the state prosecutor, and are responsible about issues of human trafficking. Those prosecutors, have gone through 3 sessions of education."( Police official, Serbia, 30 October 2014)

However, not all professionals who come in contact with victims of trafficking have received such training. The Head of a Crisis Centre in Sofia stated:

"It's a huge difference, if the police official says, leave her, she is a whore, it's another thing if the police official treats them as victims." (Service provider, Bulgaria, 13 October 2015)

A shelter coordinator from Albania acknowledged that judges and prosecutors sometimes treat victims of trafficking as any other person that comes into their courtroom:

"The judges and court are, they say that they are independent. So they are a little bit cold about the victims. "(Service provider, Albania, 23 October 2015)

According to the Shelter Coordinator, a likely reason for this behaviour could be persistent misunderstanding about what trafficking is, as well as what the victims have gone through. Thus, prosecutors are sometimes more sensitive toward victims precisely because they have had contact with them, while judges have not.

Finally, examples were provided of professionals being abusive to victims. A case manager from Belgrade expressed the view that everything that happens in the courts is "a demonstration of power". According to the same person, there have been cases of judges who, when entering the courtroom, greet the 
trafficker who is on trial and say, "Hey, X [name of trafficker], how are you?" Another judge asked a victim of trafficking how much money they took when they migrated for work abroad (and were subsequently trafficked). Upon hearing the answer of 50 euro stated, "Ha, I don't even go to the market with only 50 euro!" (Service provider, Serbia, 2 November 2015).

\subsubsection{Information and trust}

"He is still free at the moment. I just came back now, in September, from Spain. He was in Bulgaria in September. He was renewing his ID. When they checked the 3 names that he is using, it came out on the computer, that with one of those names, he had applied for renewal of his ID. How did he pass the borders? I don't know. I know he was here in September, then I don't know. I don't know how they cannot find him. How did he enter Bulgaria? How could he pass the borders? He could have passed through Romania, Serbia, Greece, but how could he enter Bulgaria again? I cannot explain that. And how could he go to the police to renew his ID? I don't have any hope that he will be captured. Especially here in Bulgaria. I don't have one ounce of trust in the police." (Trafficked person, Bulgaria, 15 October 2015)

When receiving information about their trial, trust in the professionals with whom they interact as well as trust in the system itself, are crucial factors that determine if the participation in the criminal process would have a positive impact on the recovery of the victim.

Respondents noted that it is essential that victims receive information about their rights and obligations, as well as progress of their case throughout the criminal proceedings. Too often victims do not know what will happen next, are fearful of testifying, and stressed by the suspense that the case causes. A psychologist remarked:

"Fear of the suspense is big. So we try to explain, which are the institutions that are included in the process, who cares about them. We explain that we, as the shelter, as an institution, as well as the police and other institutions, we all care about them, they gain some courage. And when these people contact them, when they visit and tell them something, that gives them courage." (Service provider, Bosnia Herzegovina, 1 October 2015)

Keeping them informed is the only way to keep fears in check. A case worker stated: 
"The victims don't understand, they think that in many cases, they are the ones being prosecuted, because of the uncomfortable situation; they have already given a statement to the centre for protection, they have already given a statement to the investigative court. They don't understand why they have to testify again, you have to explain to them why it's so complicated and hard. And then, they understand that it's them prosecuting the person. But it's not them, it's the state, and it's not their responsibility, but of the state."(Service provider, Serbia, 2 November 2015)

Another challenge for professionals throughout the prosecution procedure is gaining the trust of the victim. Such trust is often only secured through transparency and action. According to a lawyer from Albania:

"They create this trust, because we inform them continuously, so they see the progress that is being made. Being informed continuously, so they start to build this trust with us. And the link that we realise with the police or with the prosecution, they are present and they hear with their own ears. As we accompany them into these institutions."(Service provider, Albania, 23 October 2015)

A psychologist from Bulgaria notes the importance of the outcome of the criminal process to victims' state of mind:

"When there is the trial and conviction, they feel vindicated, like something that has been wrong with society has been made right. The idea of jail, is not so much about punishment, but re-education. So, when someone does go to jail, they feel vindicated."(Service provider, Bulgaria, 16 October 2015)

Prolongation of the criminal proceedings and failure to make an arrest and subsequently conviction of the trafficker are ways in which trust is lost and eroded. The bare minimum in these situations is once again to keep the victim informed:

"Because of the slowness the victims lose wish and willingness to testify. They don't have the hope to wait that the traffickers are punished."( Service provider, Bosnia Herzegovina, 29 September 2015)

"There are many questions that are always on their mind - how long will it last, will the traffickers stay in jail, for how long. When they hear that someone is convicted, then they believe in the state, they believe in the 
institutions, and the power of those institution. When the police says that we will protect you, but the trafficker is not captured yet, then there is doubt in the power of the police. When they hear that the person is arrested, then it's a big encouragement."( Service provider, Bosnia Herzegovina, 1 October 2015)

"Legal processes they tend to be prolonged, and this influences on the issue of trust to the beneficiary. Trust in the justice system, in these institutions. In some cases they regret to have made this denunciation and to have had trust in these institutions. In these cases we have even post-traumatic stress disorder...mainly because of the delays."(Service provider, Albaina, 23 October 2015)

\subsubsection{Protection from intimidation}

"His family came and they put a pressure on her, offered her money in order to withdraw the report, but how can she withdraw the report, otherwise she could be punished by the law so you know, it was a real war... We were obsessed. When somebody was coming and knocked at the door, policeman or the policeman of the periphery...We were obsessed. That is why I said, let's move from here, because we will die....And we are really calm here..."(Trafficked person, Albania, 29 November 2013)

Interviewees mentioned on numerous occasions that traffickers are not part of complex organised crime networks but often individuals who act on their own or in small groups. It may be that the trafficker and his family live in the same city or village of the victim. In these situations, the victim should receive protection not only from actual physical danger but also from 'soft' methods of pressure that can be applied in these contexts.

Unfortunately, threats and pressure are not only aimed towards the victim but also towards judges and prosecutors. According to a case worker who has often accompanied victims to court:

"The trafficker has the main word. He threatens the judge, says, I know your wife, I know your son. He says, if the judge proposes to take away his assets, he will put his house on fire...the courthouses are so small, everybody is cuddled together, the trafficker threatens the victim. ...it's rare to say, oh, wish every court process was like this."(Service provider, Serbia, 2 November 2015) 
There is no information as to what impact, if any, such intimidation can have on the criminal justice process.

\subsubsection{Not just conviction, but financial compensation}

"The government is not being...is not taking the payback from the traffickers...So she never received any penny. And there is another big gap here; the law, that in order to get some money back, she has to pay in advance $5 \%$ of this amount requested to the government...ok, I will get 20 million lek, that means 50 thousand dollars and I can pay 1\%, 2\%...Maybe I am wrong with $5 \%$ but it is a percentage... But how can I pay this percentage when I am just me? When I have felt in this kind of situation, you know. I am a victim. I don't have...otherwise I wouldn't ask. So how can I pay? This is the ridiculous part of it."(Trafficked person, Albania, 29 November 2013)

Interviewees emphasised the importance of not only convicting the perpetrator for crimes of trafficking, but also providing financial compensation for unpaid wages as well as personal trauma and suffering. As one interviewee explained, financial compensation is a "recognition that something happened to you, and that what happened was not ok."(Service provider, Netherlands, 28 March 2014)

Very few cases of victims receiving financial compensation were uncovered in the Balkans. Reasons for this could vary; victims may not be aware of the possibility for financial compensation, victims may not have the resources to finance civil action, or they may be psychologically unable to participate in criminal proceedings (in situations where prosecution is a prerequisite to action for remedies).

"Do you think they even know what compensation is? They just say, I just want him to return my mobile, and my things, that he took from me. Overall, they don't want anything from him."'Service provider, Serbia, 2 November 2015)

Often the state will refuse to use confiscated funds to compensate victims and there may be no alternative source of compensation, such as a special fund. It was noted that another important change would be to tie the process for financial compensation to the criminal proceedings, instead of requiring a separate trial: 
"They didn't want to go through the process again, it was traumatic enough up to that point. It would have been useful if that process for compensation was also part of the criminal process, so they don't have to continue. When they have the information, ok, the trafficker is convicted, but now, for financial compensation I have to go further, they rarely want to go on. Even if they are severely poor. It would help if that procedure for the criminal act, also has a decision on compensation."(Service provider, Serbia, 3 November 2015)

The Netherlands provides an example of good practice on this point. Under recent legislative changes in the Criminal Code of the Netherlands, victims may be compensated by the state as part of the criminal proceedings against their exploiter. Thereafter, it is the state that attempts to recuperate the funds from confiscated assets of the trafficker. Thus, with this new possibility, lawyers are tracking down old victims now eligible for such compensations to come and claim their money. Those working with victims have noted that financial guidance should be part of this package so that victims can receive help on managing the funds they receive as compensation.

\subsubsection{The label 'victim' and the wish to testify}

"The police came to the house, asked her information; and she said, she has information, because she knows the people. Said 'yes, I know'. And they asked her if she wants to 'say information'. And she said, of course, I want to say. And I don't speak with anybody. I need to speak. Because I was sick. I was 'banged', I was hit. So, I need to speak. Because I was closed in the house. Very long time. ...I go outside, because I cannot sit here."(Trafficked person, Netherlands, 3 August 2015)

"I lived through it. I want him to get what he deserves. However much is the law, he should be in jail, not a day less not a day more. I want an effective judgment for him. But if he is sentenced here in Bulgaria, that will not be respected." (Trafficked person, Bulgaria, 15 October 2015)

In all countries, there must generally be some identification of a person who has been trafficked as a 'victim' for them to be eligible to access protection and recovery and (re) integration services. However, this is not always an easy and smooth process. As some interviewees mention, their clients may not always want to be identified as victims. In certain cases, it takes years for someone who has been coming to a service center to finally admit that they are a victim of trafficking. Victim reluctance may be connected to their 
trafficking experience. For example, in instances where the women had some knowledge of the situations they were entering, they may not want to be labelled as victims and 'saved', but simply be provided with another job and better working conditions. Also, if the trafficker is a family member, they may not be willing to be labelled as 'victims' nor press charges due to the emotional ties.

However, there are also cases where the victims find it very important to be identified as such; in order to testify, to secure justice for themselves, and to prevent their exploiters from inflicting harm on anybody else.

"We had that amazing person saying 'now I will tell you, word by word how it happened. How he destroyed my life knowingly. Knowingly he eliminated me as a person. I will tell you everything, and then you see what you do with that information, and what kind of a decision you will take.' That was... she showed such courage. The sentence was one of the longest sentences. She told them everything. She told them that she knows, that it's not her fault, it's not her fault the trial. She said, 'what you do with this, it's on you, don't blame me, for how long the sentence will be'. ..because in Serbia, they don't have any additional prooffor the case, except for the testimony of the woman. They say, now it will be different, but I don't see how. "(Service provider, Serbia, 2 November 2015)

"She was a hero for me, the way she answered, nobody disturbed her, she was courageous, calm. The lawyer was provoking her, saying you know Serbian, why do you want a translator. And she said, I have a right to answer in my mother tongue, do you maybe want to take it away? She was right next to the trafficker, and was answering... She wanted justice to be satisfied, to put them in jail for what they did wrong. And then, she had a little girl at home, and went away to make money for her, and they tried to abuse her. So, she didn't want them to get away with that."(Service provider, Serbia, 2 November 2015)

\subsection{Conclusion}

Testimonies of trafficked persons as well as of service providers who are in direct contact with them daily give valuable insight into the issues that arise throughout the prosecution process. Certainly, criminal proceedings are not only of importance for the justice system but also have a direct influence on the recovery and (re) integration of the victims themselves. However, there 
are many problems. Trials usually last a long time and require victims to testify on numerous occasions. Each testimony is stressful for the victim and may present a possible secondary victimisation. Sharing information with the victim on progress or lack of progress in the case is crucial for building trust and ease their participation in criminal proceedings. Insensitivities on the part of criminal justice officials are not uncommon. Specialist training for criminal justice professionals is therefore crucial. Throughout proceedings, victims are often not only in possible physical danger but may experience verbal pressure from the traffickers or their family members to not testify. This must be recognised and victims must receive the appropriate protection. Although financial compensation is often a legal possibility, it is a road rarely taken in certain countries. One reason for this may be the law itself, which in Balkan countries, requires financial compensation to be pursued through a separate legal process that can only follow a conviction of the trafficker. Finally, this chapter has found that there are cases when victims are not only willing, but eager to testify to gain justice for themselves and prevent those that have hurt them from hurting other women in similar situations. These findings point to the importance of valuing victim involvement in the criminal justice process for its own sake.

\subsection{Recommendations}

The following recommendations emerge from the information presented above. First, serious attempts should be made to shorten the time of investigation and collection of evidence, as well as to expedite trials. Criminal justice processes that last up to 10 years are unacceptable. In order for this to occur, better knowledge of the phenomenon of trafficking by police, prosecutors, and judges is crucial. This would make it possible that cases are not solely based on victim testimony but other supporting evidence as well and thus quicken the procedure overall. The criminal justice system itself must also reform to make sure cases do not get 'stuck' - for example, when key officials such as the judge are moved on mid-stream. Given the impact on victims, consideration could be given to imposing a maximum timeframe, beyond which a criminal case of trafficking may not last. Finally, the criminal process should include measures for compensation or otherwise be tied to civil proceedings so as to expedite the payment of damages.

Second, measures should be put in place to minimise victim exposure in court. Ideally, victims should not be required to testify repeatedly and should be given options which protect them from further harm - such as speaking, 
writing, talking to a video camera, talking from a different room adjacent to the courtroom, etc. As far as possible, subsequent investigations and trials should use this material and not demand additional testimony. Corroborative evidence should be used as much as possible in trafficking trials to lessen the burden on the victim.

Third, victims should be kept informed of the progress of their case by their lawyers and case managers. The establishment of a relationship of trust between the victim and relevant criminal justice officials (investigators and prosecutors) is important. If necessary, they should meet with police officials in charge of collection of evidence, as well as prosecutors in charge of the case, so as to make them more familiar and hopefully build trust. Social workers, psychologists and lawyers who already know the victim well should always be present at these meetings for emotional support. In addition, at regular intervals the same service providers should request such meetings where the victim will be officially informed of progress in the case.

Fourth, it is crucial that every single person from the state system and the criminal justice system, and every service provider that may come in contact with a trafficked person has the proper training to handle such situations and communicate with victims in a way that protects their best interests and prevents further trauma.

Fifth, protection of victims throughout the trial process should be tailored to the particular situation. For example, protection needs might change depending on whether the victim is in physical danger or under psychological pressure, or both. Primarily, the trafficker and all family members should be prevented and restrained from speaking to the victim or the family of the victim, in all cases. In situations where there is danger to the life of the victim or her family, witness protection should always be possible not only within the country of origin but also with the possibility of settling the victim anonymously abroad. In cases where the traffickers are not yet arrested, the victim should be regularly informed by the police of any progress in the case, including any information on the possible whereabouts of their alleged exploiter.

Sixth, conviction of the trafficker as well as financial compensation for the victim have strong significance for the recovery and (re) integration of victims. As already noted, the Netherlands has made significant progress in this area by obligating the state to ensure compensation of the victim, 
regardless of whether or not the funds have been confiscated from the trafficker. Other countries should follow this lead. In practice, victims should be advised by their lawyers of the possibility of financial compensation, which would be more likely if ensured by the state and not solely dependent on confiscated funds of the trafficker.

Finally, while acknowledging that victim status determination is usually essential to the provision of services, entitlements, and indeed to commencement of legal action against traffickers, it is important to find ways so persons who have been trafficked are not further victimised by this label. Many victims find it difficult to identify as such and they should be left to come to this term by themselves, in their own time if they so desire. They should have the freedom to see themselves as 'victims' or 'survivors' or whichever label they prefer, if any at all. Irrespective of that choice, all should feel that the grave harm done to them is recognised and that they are not to blame. Victims who wish to participate in the prosecution of their exploiters should be given every support possible throughout the process by their case manager, psychologist or lawyer. They can serve as an inspiration not only for other victims but also for the service providers that are with them every day who are invigorated by the exhibition of such courage and strength from their beneficiaries. 


\section{Chapter 7: Sex work and trafficking for the purpose of sexual exploitation ${ }^{15}$}

\subsection{Introduction}

"I was once interviewing two prostitutes, which have been trafficked and we arrested the trafficker, and after the statement, I asked them, where do you want to go? Do you want to go to the shelter, or where should we drop you off? Well, you can drop me off at the red light district, she said."(Police official, Amsterdam, 2013)

Sex work and trafficking for the purpose of sexual exploitation have been linked since the origins of human trafficking in the form of 'white slavery' in the late 1900s (Outshoorn, 2005). Primarily, discussions have been focused on whether sex work can be a choice or if it should always be considered as forced and thus indistinguishable from human trafficking (Gallagher, 2001; Doezema, 2005). Scholars have also looked at whether the legal status of sex work has any impact on the occurrence of human trafficking (Cho et al., 2013). However, what seems to be missing is a closer look at the relationship between sex work and victims of trafficking after the trafficking experience has ended. As the introductory quote demonstrates, it is undeniable that some women after being trafficked, choose to re-enter sex work. Thus, this leads to the question, how is sex work approached in the context of (re) integration of persons that are victims of trafficking for the purpose of sexual exploitation? How do service providers and victims view the issue of sex work? What kind of implications do these attitudes have for the (re) integration process of victims? These are all questions that will be explored throughout this chapter.

This chapter will not attempt to resolve the decades long discussion on whether all sex work constitutes violence against women, nor whether sex work can be considered as a legitimate form of labour. It will simply look primarily at the way the issue of sex work is viewed by service providers, but also by victims throughout the (re) integration process of trafficked persons. The target group under discussion will mostly be Southeastern European women that have been identified as victims of trafficking for the purpose of sexual exploitation with European countries as destinations.

${ }^{15}$ This chapter will be submitted for publication as an individual paper 
As the overview of empirical literature below will show, much of current academic research that discusses both the topics of sex work and sex trafficking mainly try to show the interconnectedness between sex work and sex trafficking - the legalisation of one (sex work) leads to a rise in the other (sex trafficking). However, such studies are questionable, as they are based on large scale quantitative research that often face serious data challenges that are hard to overcome when dealing with hidden populations (Weitzer, 2015). The research in this chapter is a valuable contribution to the literature as it is based on extensive, albeit explorative, qualitative work. It aims to better understand the evolving relationship between sex work and sex trafficking in the context of (re) integration efforts of victims of trafficking.

First, this chapter will present an overview of relevant literature. A brief historical review of the relationship between sex work and human trafficking will be given as context. Thereafter, the empirical studies that try to show the relationships between the legal status of sex work and sex trafficking will be presented. This chapter continues with an outline of the methodology used and then a presentation of the main findings based on responses regarding sex work, primarily by service providers, but also by victims. Three themes have been identified and will be explored in detail: (1) sex work in the context of (re) integration following a trafficking experience; (2) views on sex work (3) sex work and sex trafficking. The first theme will explore how service providers approach sex work in the context of the (re) integration process of victims. The second theme will look at how service providers and victims view sex work, and whether it is still an issue surrounded by stigma. The third and final theme will look at whether service providers make a distinction between sex work and sex trafficking in the context of their work. The chapter will conclude with a discussion of the findings and their implications for the (re) integration process of victims.

\subsection{History for context}

The issue of sex work has been part of the discussion on human trafficking from its very inception (Doezema, 1999). Human trafficking draws its roots from a phenomenon called 'white slavery', identified in the 1900s whereby women who migrated would through that process fall victim to trafficking and be forced into sex work. However, 'white slavery' has by some scholars been identified as 'largely mythical' and a 'moral crusade' against women that have decided to migrate to do sex work (Weitzer, 2015). Similarities have been drawn between the timing of 'white slavery' of the past and 'anti- 
trafficking' movements of today as simply attempts to stop women from migrating, and in particular from migrating with the purpose of engaging in sex work. It is thus to be expected that the issue of human trafficking today would certainly draw the attention of two divergent camps of thought. The abolitionist camp does not make a distinction between sex work and sex trafficking and sees all sex work as violence against women. According to the abolitionist, the attempts to make a distinction between sex work and sex trafficking is a political strategy that tries to give legitimacy to the sex industry, and continuously and consistently violates women's rights and perpetuates a culture of gender-based domination (Jeffreys, 2009). In this direction, all sex work should be abolished.

On the other hand, the term 'sex work' was first used in 1978 by Carold Leigh at a meeting of the Women against Violence in Pornography and Media in an attempt to identify sex work as legitimate labour and try to distance individuals engaged in such labour from the stigma that surrounds the word 'prostitution' (Ditmore, 2011). Since then, the pro-sex work camp has tried to make a clear distinction between sex work and sex trafficking. Namely, sex work is defined as the "selling of sex for a fee" (Farrell and Cronin, 2015). On the other hand, sex trafficking "involves acts of prostitution that are induced by force, fraud or coercion" (Farrell and Cronin, 2015), or an act that covers "coercion, forced labor and slavery" (Butcher, 2003). Having this in mind, the abuses and violations of human rights that occur through the process of trafficking should be distinguished from the form of employment itself. In the same regard, when addressing other forms of trafficking, such as trafficking for the purpose of domestic work or forced marriage, the aim should not be the eradication of domestic work as a form of labour or marriage as an institution, but eradication of the exploitation that happens within these activities (Sanghera, 2005).

Some scholars have tried to find a so-called 'third way' accepting some of the arguments from the abolitionist camp and some of the arguments of the prosex work camp (Shelley, 2010). The third way attempts to recognise that structural factors and limited options may be what drive some women into sex work, and consequently is not the choice that they would make in an ideal situation or even in slightly better circumstances. However, it also acknowledges that women do exercise agency, even when making decisions to enter sex work, and their choices should be respected. In addition, women have the right themselves to name the sources of their own oppression, rather than defining the trafficking experience for them and should not have a 'one- 
size-fits-all' intervention imposed upon them (Shelley, 2010). In the spirit of the third way, some studies recognised that regardless of whether or not sex work can be a choice, it is work that involves significant dangers and possible harms to health, and thus these issues should be addressed and women assisted, regardless of whether or not one belongs to the abolitionist or prosex work camp (Decker, 2013). Along the same lines, a study by Saunders (2005) notes that trafficking should be seen as one of the 'occupational hazards' of sex work. Occupational health and safety concerns persist and migrant sex workers should be made fully aware of such concerns as well as informed of the forms of violence they may face.

\subsection{Overview of empirical literature}

There are also a limited number of studies that are based on empirical evidence, which try to look at the interconnectedness of sex work and sex trafficking. A study by Busza (2004), based on interviews with Vietnamese sex workers in Cambodia, tries to identify some of the impacts of antitrafficking activities on sex work. It finds that through raids, women were often arrested and would have to go into further debt to be released from prison. Further, the presence of police or NGO workers in their communities made it more dangerous for the clients, thus decreasing possible profits for the women by driving customers away. Finally, in such conditions, negotiations about the use of condoms were more difficult, and thus the health of the women was compromised to a greater degree than in conditions where anti-trafficking activities are lacking (Busza, 2004).

Based on interviews with service providers in Nepal, Worthen (2011) looks at the programmes offered by service providers to sex trafficking victims.. Worthen identifies the so called 'prostitution framework' rooted in abolitionist policies and the 'labor framework' rooted in the pro-sex work camp and notes that most of the anti-trafficking programmes implemented by Nepalese organisations belong to the 'prostitution framework'. In this regard, programmes offered do not recognise any agency that women may exercise when entering sex work. Brunovskis \& Surtees (2008) conduct an important study focused on Southeast Europe 10 years ago. It investigated the values and attitudes that are 'inherent' in anti-trafficking assistance programmes and found that assistance programs tend to 'pathologize' the choice of the woman to migrate, and entry into sex work is a way to explain this 'deviant behaviour'. They find significant stigmatisation of the issue of sex work, which this present work will show still very much persists today, 10 years 
after their study and not only in Southeastern European countries, but also in Western Europe (in the case of this work, the Netherlands).

Most recently, a body of literature has been compiled based primarily on large scale quantitative data or case studies focusing on the connections between the legality of sex work and the occurrence of human trafficking, as well as the legality of sex work and societal attitudes toward sex work. Cho et al., (2013) looks at data from 150 countries and finds that the legalisation of sex work has two effects - the scale effect, which may increase trafficking due to rise in demand when sex work is made legal, and the substitution effect, which may decrease trafficking when women enter sex work legally after sex work is made legal. The study finds that the scale effect dominates the substitution effect. Cho (2016), again using global data from 149 countries, tries to further show that liberal sex work laws do not mean greater victim protection, instead what is of relevance for victim protection is institutional quality and gender empowerment. Thus, Cho (2016) notes that liberal policy makers, tend to believe that by liberalising sex work and protecting the rights of all sex workers, they also protect the rights of those that have been forced into sex work, which is not the case. Thus, those forced into sex work are neglected, causing weaker victim protection. On the other hand, (1) commitment of institutions to anti-trafficking policies (institutional quality) and (2) female policy makers that are committed to pursuing initiatives that promote the well-being of women, ensure better victim protection overall (Cho, 2016). Jakobsson and Kotsadam (2013) use global trafficking data from UNDOC and ILO and find that the trafficking occurrence is lowest in countries where sex work is illegal - a relationship, but not necessarily a causal one. They also augment their quantitative work with two case studies of Sweden and Norway and attempt to show that it may be that harsher sex work laws lower the amount of trafficking. Such large-scale studies however have been criticised by other researchers as flawed (Weitzer, 2015) for using data that has not been standardised in order to compare countries at the global scale, all the while targeting 'hidden populations' (sex workers, trafficked). This is an issue because the country data that they use has been collected through different means (in some countries it is government institutions that provide this data, in others NGOs or international organisations). And of course, there is always the issue that some countries (and by extension their governments or local NGOs) are more efficient at identifying victims, but also in compiling such data than others. 
Another set of quantitative empirical studies use survey data to draw conclusions regarding attitudes towards sex work. Immordino and Russo (2015) use a data set from the World Values Survey to look at how sex work policy may influence attitudes concerning sex work. They find that countries where sex work is legal seem to show more tolerance toward sex work. Jonsson and Jakobsson (2017) use survey data on attitudes towards sex work from the UK, the Netherlands, Germany, France, Spain, Denmark, Norway, and Sweden. They find that people who come from countries where buying sex is a criminal act are less tolerant toward this practice. They also find that where buying sex is illegal, people who consider gender equality important are not morally accepting of buying sex, whereas those that come from countries where sex work is regulated and consider gender equality important are morally accepting of buying sex. Finally, Jakobsson and Kotsadam (2011) use a 2008 Internet survey to look at attitudes toward sex work in Norway and Sweden, as countries that have criminalised the purchase of sex. They find that people who find gender equality important, have negative attitudes toward sex work.

The work reviewed above mostly tries to show, through quantitative studies, that legalisation of sex work may be related to high trafficking occurrences. Additionally, it attempts to investigate levels of stigmatisation based on the official policy that exists in regard to sex work. What is missing is more current, in depth qualitative work, which investigates how sex work is approached and how it may influence post-trafficking situations, in both countries where sex work is legalised and where it is not. This is what the current chapter tries to address.

\subsection{Methodology}

This research is based on qualitative interviews conducted in Albania, Bosnia Herzegovina, Bulgaria, Montenegro, Serbia, the Netherlands, and Italy, from 2013 to 2015. A total of 52 semi-structured interviews were conducted, lasting from 20 minutes to 2 hours. Of the 52 interviews, 9 interviews were conducted with victims of sex trafficking, 2 with persons identified as at risk of human trafficking, 1 interview with a sister of a sex trafficking victim and 40 with service providers. Of the victims of sex trafficking, 8 were female, while 1 was male. Victims came from Bulgaria, Romania, Albania, and Bosnia Herzegovina, and they were trafficked in Italy, Greece, Spain, and the Netherlands. Service providers were from Albania, Bosnia Herzegovina, Bulgaria, Italy, Montenegro, the Netherlands, and Serbia. Their background 
and organisational role was that of case manager, children center coordinator, clinical social worker, crisis unit operator, day center coordinator, NGO or government agency director, member of female NGO lobby, SOS hot line operator, government worker, lawyer, police official, program manager, project coordinator, psychologist, psychotherapist, shelter coordinator, shelter staff, social worker, and course teacher.

Different interview protocols were used for trafficking victims, those at risk of trafficking, and service providers. The interview protocol for trafficked persons covered several general areas, focusing on 1) life before the trafficking experience, 2) the trafficking experience itself; how it happened and what helped the person to get through it, 3) how identification as 'trafficked' occurred, 4) description of the period immediately following trafficking, including questions having to do with experience of stigma and participation in assistance programs, 5) description of economic situation at the time of the interview, 6) description of social life, mental and physical health at the time of interview, and finally 7) description of how the person envisions the future. A selection of the above presented questionnaire areas was used for those at risk of trafficking, omitting any inquiry about the trafficking experience. What has been used for this research were all references to sex work by the victims of trafficking.

Service providers were asked about what they see as sex work and what they see as sex trafficking. They were also asked about their position on sex work, as well as the position of their organisation regarding sex work. Finally, they were asked to speak about cases of sex trafficking where the women returned to sex work following a trafficking experience.

The interviews on which this chapter is based have also been used for the other chapters of this thesis. However, those chapters have focused on different themes than those covered by this particular one.

As already noted in the introduction, this chapter will look at 3 distinct themes that have arisen based on the explorative qualitative research: (1) sex work in the context of (re) integration following a trafficking experience, (2) views on sex work, and (3) sex work and sex trafficking. 


\subsection{Sex work in the context of recovery and (re) integration}

The first theme will look at how the issue of sex work is approached in the context of (re) integration by service providers and by victims. Do victims share their past, which may have entailed voluntary or involuntary entry into sex work that resulted in trafficking, with others? What is the advice of service providers in regard to sharing the victim's experience with others? And what about possible return to sex work following a trafficking experience? How is such a return viewed by service providers, and why?

\subsubsection{To tell or not to tell?}

Most service providers noted that the choice of whether or not to share their past, and whom to share it with, is left up to the victim of trafficking. However, some did admit that they would recommend not to share a past trafficking experience with employers after having had experience with victims that were further exploited by their employers once they learned of their past.

Regarding the victims interviewed for this study, 2 victims noted that they would not tell anyone about their experience, in particular their family, as they would never understand or accept what had happened:

"Noooo. In my family was not, not, not, never in my life. I don't speak about prostitution. Never. So, it's not possible. No. My brother also, never not speak with me. My family is normal people. She work, everybody work. My brother work in hospital. Not doctor, only little function. My sister work in shoes factory. Also, me too, I work. And my big sister work in shop." (Trafficked person, Netherlands, 13 August 2015)

At the other extreme, 2 victims speak about their experience openly, one noting that she tells everybody what has happened to her, and the other saying she has told her family and children as they live in a small community where their experience had become known to all. What should be noted of these 2 cases, is that the woman who pointed out she shares her experience with others openly, also claimed to be drugged for the duration of the trafficking and her time in sex work. While the woman who shared her past with her children, was the case that despite being physically and psychologically abused by the trafficker, never accepted entering into sex work, and thus did not admit to being sexually exploited. Thus, one might argue that it is easier 
to share the experience if the victim finds a strong justification for its occurrence or completely distances herself from it.

Between the 2 extreme groups, is the largest group of 4 victims who remarked that only their family knows about their past. 2 of these victims were accepted by their family, while 2 were rejected and had not reconciled. Finally, the male victim noted that although he is open about his experience, willing to share what happened to him, and talk about it with researchers, he is more reluctant about sharing it with friends or acquaintances, and even less open to sharing it with family:

"How you going to tell your parents that you were a prostitute. How you will tell your parents that you know it was a problem to do, and you don't mind it. How you tell them at this time you were kind of happy, because the job was not heavy for you, in a psychological way. "(Trafficked person, Bulgaria, 14 October 2015)

Thus, it seems that the largest group of respondents follow a particular pattern of disclosure characteristic of persons that may feel stigmatised - they divide the world into a larger group to which they do not disclose their experience, and a smaller group that they may trust, to whom they do disclose their experience (Goffman, 1963).

\subsubsection{Return to sex work?}

It was noted in the introduction of this chapter that it does occur, although it is not possible to say how often that women identified as victims of sex trafficking, return to sex work following the trafficking experience. This raises the question, how is such a return to sex work following a trafficking experience viewed by both service providers and victims? And further on, how is the overall (re) integration of victims that return to sex work evaluated by service providers?

When asked if they would consider re-entering sex work, all the victims interviewed for this study, answered negatively. When service providers were asked if they would consider a woman that goes back to sex work after a trafficking experience as a success case of the recovery and (re)integration process most also answered negatively. The reasons given for such answers, both by victims and service providers, were similar and mentioned that what is needed after a trafficking experience is a return to the 'normal' and sex 
work does not satisfy this need. In addition, it was noted that the return to sex work means exposure to the possibility of exploitation once again.

One victim voiced the need to feel 'normal' in the following way:

"I think I need work. And I am happy, because now I can start in this restaurant. Maybe it's two times, you know two times per week, but its ok, because after this, I can have you know, I can stay with normal people, I can do some normal stuff. (...) I said myself, maybe, I don't know, good job. Let's say this, good job, to stay to the floor [to stay grounded]. To little bit, to be normal life, you know. Little bit. Change.' (Trafficked person, Netherlands, 13 August 2015)

A service provider similarly stated:

"And the re-trafficking, or prostitution, is a thing of failure of (re) integration, (re) integration failure I think. Because if a victim of trafficking, she is going to prostitute, or re-trafficked again, that means that she has not the sources to live in a normal way, I think, with their family. "(Service provider, Albania, 23 October 2015)

Exposure to violence and possibly falling victim to exploitation once again, was perceived by both victims and service providers as another reason why a return to sex work following a trafficking experience would not be recommendable:

"Because it's a dangerous job. And if I do this problem for these people, if I work again in sex job, she [trafficker] meet me everywhere. In internet."(Trafficked person, Netherlands, 13 August 2015)

"I really think that this is a big risk. Especially, here, there are many different risks. Risks of re-trafficking, to different types of risks, health wise and other. Risks from violence, as such. So, there have been girls that were talking about eventually doing that, because it would be the only way to make money, but we always try to emphasise the risk factor. Especially because of the experiences that they have had, and the general violence that in our society dominates, and which certainly, if it doesn't come to trafficking again, we think they are in risk of other types of violence. "(Service provider, Serbia, 30 October 2014) 
Service providers also emphasised the attempts they make throughout the recovery and (re) integration process to direct women towards the 'right path' or direct them in the 'right way', which would not include returning to sex work:

"Of course, we do not judge such persons, who even after trafficking, and recovery, enter the world of prostitution. We accept them, before anything else, as human beings who need help and understanding, and a conversation that tells them that they are again in big problems and they should try to come out of them, with professional help, and the help of humanists who deal with this, and understand this."(Service provider, Montenegro, 7 December 2015)

"Our work as a group is to show her the right way. So, by providing her with all the services, is to show her the right way. Then is the choice of the person, if she chose either this path, or this one. But this cannot be considered as our success or failure, but as the choice of the person herself. So, it's either her success or her failure. But even, but we can say even this, even when she decides so to go back, so to be, we can say a prostitute, maybe there are different reasons why she does this, because of the vulnerable situation, where she might be, because she might not have the family support, and because of her economic situation, she has no support from the state."(Service provider, Albania, 23 October 2015)

"So, the moment that they want to continue their life, not staying in this shelter all the time, but when they say, I can't stay all the day without doing anything, I want to work, I want to find something, then it's this point, this very important point, that the people here try to direct them in the right path."(Service provider, Albania, 25 November 2013)

It should be pointed out, however, that in situations where the women persisted in their decision to remain in sex work, either due to financial reasons or in some instances mentioned by responders to finance an addiction such as alcohol and drugs, service providers did not pull away their support but still tried to assist in the limited ways they could:

"For me, this is acceptable. Because I think that it is a matter of choice. However, it should be said that in some cases, it is not said, it is kept quiet. So, we know they are prostituting themselves, they just openly tell. However, our victims know that although we know that, they cannot count on our support, to free them, in case they are arrested by the police, for prostitution. 
This we cannot do, because it's a criminal act, for which they would be responsible, and have to answer for it. But, we offer them emotional support and advise, if they want it. We have a couple of cases, a victim that we know is still in prostitution, she was already in prostitution before the trafficking experience. She requires our help only as far as the participation in the criminal trial against her trafficker goes. Otherwise, she doesn't want us to meddle in her other parts of life. Which for us is acceptable. We are giving advice and direction, but for what they don't want us to comment, we don't. We have it in mind, if there is a moment when she wants to work on it, ok. Otherwise, we do not judge, but we also don't solve it without her." (Service provider, Serbia, 3 November 2015)

"Our organisation, we are very liberal. In these cases, what is important, when someone works in this, we would say, it is okfor us. I mean, it is not ok, but we try to understand. And we ask that she tells us where it happens, how it happens etc. And we try to work with her again, to work through everything, to see how to take her away from that. But, we would not judge her. That is key. We would understand her. When they are in the shelter, they are banned from being in prostitution, while accommodated with us. But, it often happens. The permanent decision about exiting, it's like with addicts. It happens very often. A lot of them go back to it, as a source of income etc." (Service provider, Serbia, 2 November 2015)

To summarise, when considering whether or not to expose their past to others, victims of sex trafficking in this study seem to follow a pattern typical of persons that feel stigmatised - only sharing their past with a very small group who they trust and hiding it from the rest of society. In addition, the return to sex work following a trafficking experience is often seen as a failure of the (re) integration process by service providers. Part of the reason is that sex work involves many dangers and may put the victim in a situation of exploitation once again. However, both victims and service providers view sex work as something that is not 'normal' and service providers make attempts, throughout the (re) integration process, to set victims on what they see as the 'right path', which means away from further sex work. Once again, these are all attitudes that show sex work to be a source of stigmatisation.

\subsection{Views on Sex work}

Stigmatising attitudes toward sex work were outlined in the preceding section, in the context of discussing sex work with victims and service 
providers, as it relates to the (re) integration process. However, existing and persisting stigma toward sex work was also discussed more openly with service providers, as well as identified when expressed more subtly through their references toward the stigmatised group - in this case female sex workers.

Primarily, service providers openly observed that stigma goes hand in hand with sex work and sex work is a profession that is highly stigmatised by society, even in places where it is legal:

"The problem is that there are still a lot of stigma around prostitution. So, I think if you are choosing to do this work, it doesn't matter what your reasons are, isolation is always difficult to have a comfortable life, if you have to do the work you are doing.(...) Then it's also very difficult, because you have a lot of, you have to get a bank account, or you want to finish your rent, or you want to buy a house, it makes it all more difficult. So, it's a little bit difficult in the Netherlands, because we legalised it, but it's still a difficult subject."(Service provider, Netherlands, 4 November 2013)

However, what is perhaps of even greater importance are the more subtle, personal vocalisations of stigmatisation of sex work that were expressed through the references made by service providers towards sex workers and sex work. Although some service providers noted that some of the character traits of sex workers are strength because they are used to harsh conditions and must learn to survive, having power, and being freer than 'we are', the character traits that were expressed with negative connotations were numerous. Namely, respondents described sex workers as flirtatious, looking for thrills and high adrenaline, lazy, manipulative, promiscuous, and prone to blackmail.

Service providers also characterised sex work as not being a 'normal' profession and not a way to make an 'honest living':

"We still don't think it's a normal profession. So, it will always be difficult. So, as long as we don't accept it as a society that it's a normal profession, then it's really difficult to get accepted."(Service provider, Netherlands, 4 November 2013)

"She is trying a project now, for women to get work experience and eventually they can get a job in another line of work and make an honest living, not as 
much as she would earn as a prostitute, but..." (Police official, Netherlands, 4 November 2013)

"The salary they would earn from an "honest" job, let's say, is very low. It always helps them with the payments, the house, the children." (Service provider, Albania, 27 November 2013)

Service providers often tried to explain why women would be sex workers. Some of the respondents who believe that sex work can never be voluntary noted that women became sex workers because of structural reasons and a society that would not give them any other option. Or, from the same group of respondents who did not believe sex work can be a choice, it was perceived that it was always due to the existence of some form of sexual exploitation in the woman's past. In these cases, women may become promiscuous as a way of gaining back some control in their life. In this regard, for some women sex work becomes the only thing they know. However, other respondents also saw sex work as a trap, where once captured, it would be very difficult to exit largely due to getting used to a 'lot of money':

"How do you say it, prostitution is a ... a lot of the girls think, I do this for a short time, and then I have enough money, and then I can go home, or give to my family, or go to school. But they earn more money, they spend more money, they stay in prostitution, they need stuff to do the work, and it's a trap. That is the word. It can be a trap. And that's a big danger I think."(Service provider, Netherlands, 28 March 2014)

"There are women who can perform this action and they are able to separate love from sex, they are able to act like a mechanism just to get the money. And I think that's the biggest issue, is the money."(Police official, Netherlands, 4 November 2013)

Stigma towards sex work was expressed also by the victims interviewed for this research. Once again, what is most prominent is the view that sex work is not something that is 'normal':

"Because I see what is prostitution, it's not for me. I like job, normal job. (...) prostitution, this is not one job for old woman, or is not job for young woman normal."(Trafficked person, Netherlands, 13 August 2015) 
"They all say, 'I am voluntarily doing it'. How can you be doing it voluntarily, it is not normal for me. But, they don't know. According to me, they simply fall in love with the pimps." (Trafficked person, Bulgaria, 15 October 2015)

In addition, some of the victims interviewed for this study tried to present reasons as to why they may have entered sex work knowingly. Namely, two respondents noted that they would have never considered being sex workers if it were not for financial reasons - for one it was to pay for an electricity bill, for the another it was to provide milk for her infant.

The remaining respondents did not remark at any point that they were willingly entering a profession such as sex work and made attempts to go a step further and distance themselves from sex workers and sex work. In this regard, one respondent noted that she was drugged and does not remember anything related to the experience of being a sex worker. Another respondent was physically forced to be a sex worker, while yet another victim claimed that despite being severely physically and mentally abused, she never became a sex worker. In one instance, a victim made a reference to female sex workers as 'whores'.

It was only the single male victim of sex trafficking that did not try to distance himself from the experience of sex work, stating that:

"It was really funny, because people really liked me. In this moment, I fixed a lot of my debts. It was really good for me. And maybe because I was young, maybe because I was hungry for money, or I don't know how to say, I wanted more more more. It's like drug addiction, when you are good in something, you want to do it again, and go more and higher, and things like that."(Trafficked person, Bulgaria, 14 October 2015)

It seems that even outside of the context of (re) integration, sex work is heavily stigmatised by both service providers and victims alike. It is not seen as 'normal' and as an 'honest way' to make a living. Service providers stereotype sex workers as lazy, manipulative, and promiscuous. In addition, both service providers and victims try to justify entry into sex work, even when such entry was done knowingly. 


\subsection{Sex work and sex trafficking}

Finally, this last theme explores the view of service providers regarding distinctions, if any, between sex work and sex trafficking, and is reflected upon in light of the current debates on these issues between the abolitionist and pro sex work camp.

According to one group of respondents, sex work can never be voluntary. However, the reasons that would push women into sex work vary. According to an interviewee, even when a woman says that she has voluntarily entered sex work, it is not voluntary. The reasons being a system and a society that has forced her into the choice because she cannot find a job, take care of her family, and provide the basics for her children:

"For me, prostitution, a woman that 'voluntarily' is in prostitution, it is not voluntary. Because if we lived in a healthy society, a society where you have possibilities, this would not happen." (Service provider, Serbia, 2 November 2015)

However, for another respondent the reasons sex work is never freely chosen is because women that enter sex work are usually victims of sexual violence prior to entering sex work. It is being a victim of sexual exploitation that has led them along one of two paths, being promiscuous or avoiding any sexual relationships:

"According to me, girls that have been victims of sexual violence, go into prostitution. And this is a way to keep control. They had lost control, before, so they now try to get back that control, and they want to say 'I am deciding to do this'. It is not uncommon, that girls that have been victims of sexual violence, have promiscuous behaviour. They either stay away from sexual relationships, or they are promiscuous, and become prostitutes. (...)So, they have the belief that they are not good for anything else. They don't have anybody to teach them about anything else."(Service provider, Bulgaria, 16 October 2015)

Still, it should be noted that one of the interviewees, while still presenting the view that sex work is never voluntary, did make a slight distinction between sex work and sex trafficking: 
"There are cases where a girl that works in prostitution, is forced...she realises that she has to behave a certain way with the customer, because he paid, the sexual experience is his personal choice. So...this also happens, in prostitution. But, in human trafficking, she has to additionally bare the trafficker. That her freedom of movement is not there, that she is blackmailed, that in case she does not do something that they are asking from her, her family will be killed. Or that she will be killed. Both cases, the problems are very complex."(Service provider, Serbia, 2 November 2015)

A second group of respondents noted that although they do see a distinction between sex work and sex trafficking, society does not. The impression given by this group is that regardless of whether the two phenomena differ, society and the communities where the victims return, as well as many families of victims, see no difference. Thus, distinctions that may be made become irrelevant, especially when placed alongside the stigmatisation that victims face from their surroundings due to equating sex trafficking with sex work. It should be noted that it is implied in these statements that respondents believe society sees sex work as something voluntary, immoral, condemns it, and thus when associated with sex trafficking, the stigma it gives rise to has a negative impact on recovering victims:

"According to me, most people look at victims of trafficking, as prostitutes. They don't understand that this is something forced, and women didn't want that to happen to them. And according to people, if a woman wanted to be a prostitute, then this is a reason why trafficking happened (...) This is sad."(Service provider, Bulgaria, 16 October 2015)

"Well, here in Albania, it is, if the people know that you were trafficked, they label you prostitute, not only we can say the relatives, the neighbours, the people that surround you, but even your family. In most of the cases, family members, they do not accept their daughters back home."(Service provider, Albania, 23 October 2015)

Finally, a third group of respondents made a distinction between sex work and sex trafficking by noting that sex work may be voluntary and can be a choice, while sex trafficking is a criminal activity that implies the use of force. Even if a woman had entered sex work because she did not see any other alternative, it was still seen as her choice, while sex trafficking was a criminal act that was done through force and violence: 
"The difference is that the trafficking is a criminal thing and can operate through criminal cases, though the judiciary. The prostitution is the activity that is free, must be free. It is not a victimisation of people."(Service provider, Albania, 23 October 2015)

It was mainly within this group of respondents that the term 'sex worker' was used:

"Prostitution, is especially, is a profession that she does willingly. She is a sex worker. It's her choice. Meanwhile, the victim of trafficking suffered from this. While a prostitute she does this profession because she wants to get incomes from this profession."'(Service provider, Albania, 23 October 2015)

To summarise, three distinct groups of service providers were identified based on the distinctions they made (if any) between sex work and trafficking for the purpose of sexual exploitation. Although one group of respondents said that they clearly distinguish between sex work and trafficking, the other two either remarked that they do not make any distinction or that whether they make a distinction or not is irrelevant when you consider that for society, the two concepts are one and the same.

\subsection{Discussion}

The goal of this chapter was to explore how the issue of sex work is viewed in the context of (re) integration processes, how sex work is viewed in general as well in comparison to sex trafficking, and on the basis of that, derive what kind of implications this may have for the (re) integration of persons trafficked.

Based on the results presented in theme one, a return to sex work following a trafficking experience would be considered an undesirable outcome of the (re) integration process. Thus, service providers expressed their attempts to 'set victims on the right path', which means away from prostitution. This is in line with research conducted in Nepal, which found that service providers working in anti-trafficking followed more a 'prostitution framework' rooted in abolitionist policies in their (re) integration efforts, rather than a 'labour framework' rooted in the policies of the pro-sex work camp (Worthen, 2011). It also resonates with previous research on the topic in Southeastern Europe (Brunovskis \& Surtees, 2008). Abolitionist views on sex work were expressed by service providers also in the third theme, where one group of respondents 
did not recognise any distinctions between sex work and trafficking and another noted that society does not make such distinctions, so their own personal view is irrelevant. One of the reasons already mentioned by service providers, in the context of this study, for not wanting victims to return to sex work was the health hazards such an environment may pose. Once again, that sex work is a dangerous working environment has been recognised by other research in the field (Saunders, 2005).

However, the main overwhelming finding of this work is that sex work is stigmatised by both service providers and victims, openly and subtly, whether in the context of trafficking or outside of it. This disproves the assumption that service providers working in anti-trafficking would not exhibit stigmatising attitudes toward sex work. Unfortunately, this corroborates with research from other fields as well, such as of health care professionals who interact with patients that live with HIV and who stigmatise their patients, even though they may have good intentions overall (Sutterheim et al., 2014). Sex work has been labelled as 'not a normal profession' and 'not an honest way' of making a living, by respondents. Sex workers have been stereotyped as lazy, manipulative, promiscuous, flirtatious, and prone to blackmail. Such distinctions between what is seen to be 'normal' and 'not normal' and the construction of stereotypes which are then linked to the label of sex worker are the very basis of a stigmatisation process (Goffman, 1963; Link \& Phelan, 2001). In addition, both service providers and victims regardless of whether the entrance into sex work was conducted knowingly, try to explain and justify that entrance through the creation of 'stigma theories' (Goffman, 1963 ) - in the examples of this chapter, these were structural factors, financial reasons, or in some cases being drugged.

The fact that victims tried to distance themselves from the sex work experience and in some way explain or justify it through the creation of stigma theories may also suggest the presence of self-stigma (Bos et al., 2013). The occurrence of self-stigma in regard to sex work may also be traced in the majority of victims preferring to share the trafficking experience in which they may have been involved in sex work with only a small group of people they trust and try to hide it from the rest of the world. In addition, service providers sometimes advised victims to not disclose their past to employers. Also, as already noted, some pointed out that society does not distinguish between sex work and sex trafficking- in that, if victims of sex trafficking were exposed, they would be viewed as sex workers and blamed for what had 
happened to them. Such attitudes may show the presence of both public and structural stigma concerning sex work (Bos et al., 2013).

Also interesting, however with a note of caution due to the small sample of respondents, is that in the context of this study, stigmatisation of sex work was still expressed and evident by respondents coming from countries where sex work is not illegal (the Netherlands and Bulgaria). This is contrary to research presented at the beginning of this chapter, which has shown more tolerant attitudes toward sex work in countries where sex work is legal (Immordino \& Russo, 2015; Jonsson \& Jakobsson, 2017).

What does all this mean for the (re) integration process of trafficking victims, in particular for their recovery? As discussed in Chapter 4 of this thesis, recovery in the context of trafficking should be seen as a process that is a part of overall (re) integration. Two aspects of recovery were identified - physical and psychological, but it is the psychological recovery of victims that is relevant here. Psychological recovery was seen as having two dimensions individual and social. The individual dimension required that the self-esteem of the victim is restored, while the social dimension of recovery meant that the victim is capable of forming new, healthy relationships based on trust (Harvey, 1996). However, the occurrence that sex work is heavily stigmatised and victims often self-stigmatise, may mean that full recovery would be difficult as one of the consequences of stigmatisation is low self-esteem.(Bos et al., 2013; Major \& O'Brien, 2005). In addition, one could argue formation of new and healthy relationships built on trust would be difficult if the victim fails to fully reveal their past to those closest to her, out of fear of being stigmatised and blamed for her experiences.

The relevance of trust and the presence of stigma in this context is significant not only in regard to non-institutional social relationships that the victim forms following a trafficking experience, but also in regard to the relationships built specifically with service providers. The importance of such relationships has been discussed in detail in Chapter 5 of this thesis. However, the establishment of such relationships would seem difficult if victims perceive the stigmatisation expressed by service providers in regard to their involvement in sex work.

A group of respondents not captured by this study was in fact victims that return to sex work following a trafficking experience. Their perceptions in regard to sex work, as well as regarding how sex work is approached and 
handled throughout the (re) integration process of trafficking victims may be significant in reforming and improving anti-trafficking efforts, and is a good direction for future research.

Finally, an additional direction for future research may be to look at the experience of male sex workers and male victims of trafficking for the purpose of sexual exploitation. The one interview conducted for this thesis indicated that there may be significant differences in how men and women approach sex work. The experiences of the male interviewee of the (re) integration process following the trafficking, also largely differed from that of the women interviewed for this thesis. However, there were also significant similarities, such as the feeling of stigma. Of course, conclusions cannot be drawn from this, and thus further exploration of male experiences of sex work and sex trafficking would be necessary. 


\section{Chapter 8: Conclusion}

\subsection{Introduction}

As noted at the very beginning of this dissertation, human trafficking has become one of the major human rights issues of the present-day. The groundwork of how human trafficking is defined today, the basis of how to approach it and deal with it in the context of international law, was laid down in 2000 with the introduction of the United Nations Protocol to Prevent, Suppress, and Punish Trafficking in Persons, Especially Women and Children. Legislation at the national level in countries throughout the world followed as states ratified the Protocol and incorporated its recommendations in their own national legislation. Then, there was the wave of assistance programmes for victims, introduced and implemented by international organisations, as well as smaller non-governmental organisations. Some discussions in the context of all these developments have questioned the very existence of human trafficking, instead remarking that it is just a way to scare women away from migrating or entering sex work (Andrijasevic, 2007; Weitzer, 2007). There is also ambiguity when it comes to numbers - what is the scale of this issue? A question that remains unanswered, although new attempts at estimation are ongoing.

But are all these doubts that surround the issue and its scale really relevant when it comes to helping persons that have been identified as 'trafficked', meaning some form of exploitation has clearly occurred? The answer is no, even if the numbers that pertain to trafficking are limited to those identified as trafficked, which is around 50000 (UNODC, 2016). In this regard, academia has been lagging behind in terms of research that would address the issue of (re) integration, not only in the short term but also in the long run, of persons that have been trafficked. The goal of this thesis is to contribute relevant research to fill this gap - what are the short-term and long-term factors influencing (re) integration and what are the dynamics of their interactions and relative influence and importance, in the short and long-term.

This final chapter will begin by (1) referring back to the main research question and the relevant sub-questions to discuss the main conclusions of each of the individual chapters. Thereafter, it will outline the (2) limitations 
and implications of this particular research, general and methodological, (3) theoretical and practical implications, and finally, some (4) recommendations for future research.

\subsection{Main conclusions}

The principal research question that has been addressed throughout this thesis is:

\section{What determines successful recovery and (re) integration of women trafficked for the purpose of sexual exploitation, and how do influencing factors interplay, in the short and long run?}

Chapter 4 discussed the concepts of recovery and (re) integration and what could be considered a success or failure of the process. Chapter 5 gave an overview of the factors which influence the (re) integration process in the short run and long-term, how those factors interact, and which factors may be more important than others. Based on initial literature research and the pilot study, two other themes were investigated which are found to be relevant for the (re) integration of trafficked persons. Those topics are covered in Chapter 6 , focusing on the importance of prosecutions and trials against the trafficker for trafficked persons and finally, Chapter 7, which looks at how sex work is addressed in the context of (re) integration. The main conclusions of each of these chapters follows.

\subsubsection{Recovery and (re) integration (Chapter 4)}

The main goal of Chapter 4 was to discuss how to frame the concepts of recovery and (re) integration in the context of trafficking. The concept of recovery in the context of trafficking was approached by applying and referencing psychology literature on the more general topic of recovery from trauma (Brown et al., 2011; Masten, 2007; Nicola et al., 2012; Tummala Narra, 2007; Ungar, 2013; Wright et al., 2003). It was concluded that for the purpose of this thesis, recovery would be considered as a process that is a part of the wider (re) integration efforts, which may begin at the same time as the overall (re) integration and last throughout the (re) integration process. Recovery from trafficking may have two aspects - physical recovery from any physical injuries accrued throughout the trafficking, and psychological recovery. Psychological recovery in turn can have two distinct dimensions (1) individual - having authority over the remembering process, self-esteem and 
self-cohesion, meaning making, and recovery from PTSS; and (2) social - the ability of the trafficked person to form social relationships (Harvey, 1996). (Re) integration as noted would be an umbrella term which contains the concept of recovery. In addition, on the basis of empirical findings of this thesis, (re) integration would have 3 other dimensions: (1) economic - having a place to live, having financial stability, and sustainability; (2) institutional institutional functioning, coordination, cooperation in offering support; relationships built with service providers and peers; legal empowerment in acquiring documents which give access to further support and additional services and finally (3) social - reconciliation with the family of origin or the family created before the trafficking experience, creation of a new family. Although this is not an exhaustive list of elements which could be considered in evaluating the success of the (re) integration process, they are nonetheless in line with other research (Surtees, 2010).

Chronologically, some outcomes of the (re) integration process should be aimed at and achieved soon after a person has been identified as a victim of trafficking. The most urgent needs, as already elaborated upon through this thesis are psychological and physical recovery. Thus, if a victim is suffering from PTSS this should be addressed. In addition, any urgent physical injuries should be attended to. It is after these needs are met, that the survivor can work toward establishing and re-establishing social relationships, and ensuring her financial survival in the future.

However, the most significant contribution of this chapter, was not in listing elements of successful (re) integration. It was in trying to understand, based on feedback from service providers who directly work with trafficked persons, whether a (re) integration process should be evaluated as successful only if progress is seen across all the various dimensions of (re) integration. What this research found is that success can be observed when there is positive progress along any of the different dimensions of (re) integration. Thus, in the context of this thesis, there will be no definition given as to what a clear case of successful (re) integration is. Rather, the discussion will be about the continuum of success or continuum of success along different dimensions. These conclusions are consistent with literature on recovery from complex trauma (Nicola et al., 2012).

To conclude, although in this thesis recovery is clearly conceptualised, elements of successful (re) integration are clearly identified - what successful (re) integration is, will not be defined. The reason being, that we cannot speak 
about clear cases of success in the context of (re) integration of persons trafficked for the purpose of sexual exploitation. What can be discussed is the continuum of success and success along one or more of the different dimensions of (re) integration.

\subsubsection{Autonomy versus independence (Chapter 4)}

Some literature on (re) integration success places significant importance on the survivor gaining independence and being self-sufficient (Surtees, 2010). However, one of the frequently given answers by service providers, when asked what they consider successful (re) integration is, was when the person had married and formed her own family. At first, this finding would seem to clash with existing literature. However, what may be causing the seeming disagreement is a confusion of the terms 'independence' and 'autonomy'. Such confusion of terms is not uncommon in psychology literature (Ryan et al., 2016). Studies on human development emphasise that what should be desired is for a person to be able to practice their autonomy in decision making and not necessarily their independence (Ryan et al., 2016).

In the context of (re) integration following a trafficking experience, this seems to be of particular importance. An example was given where a victim was financially dependent on her partner. What should be looked at in this situation is not the issue of financial dependence, but the relationship between the survivor and her partner. Is it a relationship in which she has entered through her own decision? Is she receiving emotional support that she needs? Does she have autonomy within that relationship? If the answer to all of the above is yes, then such a situation, even though it may involve financial dependence on someone other than the victim herself, could be considered a positive influence on the (re) integration process. An additional reason for this would be one of the primary outcomes of this research regarding what factors are more important than others in (re) integration processes. Namely, the building of healthy relationships and the establishment of a supportive social network, be it based on personal contacts or a close relationship with service providers, are crucial for long-term (re) integration. These are points discussed in the following sections.

\subsubsection{Healthy relationships (Chapter 4, Chapter 5)}

The ability to form healthy, personal relationships in which the survivor can practice her autonomy, and relationships with service providers built on trust - to give two examples- are the most significant factors that could contribute toward successful long-term (re) integration of trafficking victims. Of course, 
for this to be achieved and for the survivor to have the ability to re-establish healthy relationships, other factors are also important upon being identified as a victim of trafficking, especially in the short term. Thus, therapy is crucial, as well as being given clear, open, and honest information from service providers on immediate steps that follow after being identified as a victim of trafficking. Such exchanges would work towards re-establishing trust that the victim has lost in social relations.

However, the importance of the ability to build healthy social relationships should not be taken to mean that long-term progress regarding (re) integration is impossible without such relationships. Thus, there may be cases where financial independence and economic security have been achieved and the trafficked person may be in a stable, sustainable situation where she is not being exploited or in danger of being exploited again. On the other hand, such situations may be more fragile than those where the survivor has established a social safety net, rather than just a financial one. Economic empowerment is important, and is a significant finding of this research, as well as other research on the topic (Surtees, 2010, 2013; Sharma, 2015). Nonetheless, in an environment of economic poverty, which many of the women that are victims of trafficking come from, and the general economic insecurity of today's world, economic empowerment as important as it is, may still be less so than the social aspects of the (re) integration process, at least as far as long-term sustainability is concerned.

In addition, the importance of building healthy social relationships may be a fulfilment of the belongingness need (Leary \& Baumeister, 1995). According to the belongingness hypothesis, "human beings have a pervasive drive to form and maintain at least a minimum quantity of lasting, positive and significant interpersonal relationships" (Leary \& Baumeister, 1995, p. 497). Some of these relationships may also be formed in times of crisis (Leary \& Baumeister, 1995), which is of particular importance in the context of (re) integration of a trafficked person, and regarding the bond created with service providers. The breaking of such bonds in the future or the overall lack of such bonds may cause mental and physical illness (Leary \& Baumeister, 1995, Leary, 2010). Thus, for victims of trafficking it may be of special importance to make sure that the ability to form positive interpersonal relationships is established or re-established following a trafficking experience. Many trafficked victims reported having lost trust. The recovery of trust would be one of the components necessary to begin rebuilding the capacity to connect to other people in a healthy way, which preserves their autonomy. This is 
consistent with literature on the recovery from sexual assault, which points to the importance of establishing trust, in order to benefit from relationships with family members as well as mental health professionals (Billette et al., 2008; Campbell et al., 2001; Glass et al., 2000; Starzynskia et al., 2017).

This thesis identified two types of relationships which may be significant for preserving successful (re) integration in the long run - relationships established when forming a new family following a trafficking experience and relationships built on trust with service providers. Both types of relationships will be discussed in the next sections.

\subsubsection{Personal relationships (Chapter 4, Chapter 5)}

The importance of the family of origin or of families that the victim had formed prior to the trafficking experience have been recognised as a very significant factor influencing (re) integration by research up-to-date (Surtees, 2010, 2013; Brunovskis \& Surtees, 2012). The challenges in family reconciliation have been identified, crucially in situations where the family, in particular children, may not have understanding for the the victim's experience. However, this thesis has found that when reconciliation efforts go well, they have an overwhelmingly positive influence on the (re) integration process of the trafficked person. This is also consistent with literature on the recovery from sexual assault, where women who involved their spouses in the process of their recovery showed more progress (Billette et al., 2008). Children were often found to be a source of strength and motivation for the women that were interviewed for this research, a force that pushed them to go on and survive.

A development that has received less attention in studying social relations of trafficked persons following a trafficking experience, has been the formation of new families, which may or may not be aware of the background of the victim. It has been the finding of this thesis that when new families are formed and women have found supportive partners, entered new relationships where exploitation and abuse does not occur, and where both individuals are on an equal footing, this development may have one of the most significant positive influences on the sustainability of (re) integration processes in the long run.

The ability to build healthy relationships, in which the survivor is capable of making her own decisions and practicing her autonomy, are key. The outcome of this process could be marriage, which was listed above as one of the dimensions of successful (re) integration. Thus, it was emphasised throughout 
this thesis that service providers often noted that they believed successful (re) integration has occurred, when the victim has married and formed her own family. However, the importance given to marriage should not be taken to mean that a woman is incapable of (re) integration without the help of a partner. Instead, marriage in this context may be better understood as a symbol - a symbol of a partnership that embodies a healthy relationship and which through marriage, is given a sort of permanent status (Leary \& Baumeister, 1995). Thus, a partnership, often solidified through marriage, may be seen as a way to satisfy the need for-long-term belongingness, and thus contribute towards long- term (re) integration (Leary \& Cox, 2008).

Chapters 5 through 7 also touched upon the issue of whether women expose their past to friends and family. Literature on recovery from rape has found that recovery tends to be more successful when survivors adopt expressive coping strategies, rather than avoidance coping strategies (Burt \& Katz, 1988). In the context of trafficking, this remains uncertain. There were cases reported and discussed in Chapter 7: Sex work and trafficking for the purpose of sexual exploitation, where women shared their experiences with friends and family, and this had a positive influence on their (re) integration, and cases where women preferred not to share their experiences, and again, this did not necessarily prevent them from moving on with their life or forming new relationships. Again, literature on recovery from trauma points to the benefits of being open in new relationships in order to be able to solidify the bond (Burt \& Katz, 1988). This may not be necessarily the case when it comes to victims of trafficking. The reason may be the existence of strong selfstigma and public stigma in cases of trafficking where the victim of sex trafficking is often indistinguishable from a sex worker, and sex workers themselves are the target of severe stigmatisation (a topic covered in more detail in Chapter 7). Thus, it is not just the trauma preventing victims from exposing their past, but also the fear of stigmatisation. In this regard, an establishment of a new family also promises a new beginning. The new families may also satisfy the need of victims to be in a social environment unfamiliar with their past, which works well for some victims.

\subsubsection{Relationships with service providers (Chapter 5)}

If the possibility of being in an environment that is unaware of their past is one of the positive aspects of forming new families for victims of trafficking, keeping in touch with service providers who are aware of their past and with whom they can always discuss it, may be one of the positive aspects of sustaining relationships between the survivor and service provider. This is the 
other type of relationship identified by this thesis, which has a clear positive influence on the (re) integration process.

One of the stronger recommendations of existing research has been to implement individualised assistance programmes throughout the (re) integration process of victims (Brunovskis \& Surtees, 2012). One way to achieve that is to be able to get to know the victim, talk to her, and hear what her needs and wants for the future are. Thus, building a relationship between service provider and victim is crucial. Additional research has identified the importance of trust in the initial exchanges between victims and service providers (Brunovskis \& Surtees, 2012; Walsh, Black \& Koser, 1999; Koser $\&$ Pinkerton, 2002). Some of the research mentioned puts emphasis on trust persons have in the information they receive from service providers (Walsh, Black \& Koser, 1999; Koser \& Pinkerton, 2002). Others note that trust in the service provider is crucial when women decide if they want to accept assistance or not (Brunovskis \& Surtees, 2012). Research focusing on recovery from sexual assault, also identifies the importance of building a strong relationship with mental health professionals, which is based on trust, for the recovery process. (Campbell et al., 2001; Glass et al., 2000; Starzynskia et al., 2017). It also recognises that victim blaming can have a significant negative influence on recovery (Campbell et al., 2001; Glass et al., 2000; Starzynskia et al., 2017).

Thus, based on existing research, relationships based on trust between beneficiary and service provider are significant in the immediate aftermath of being identified as a victim of trafficking. However, although the relationship is important in the initial phases of (re) integration, where future steps are decided, what this research has found is that this relationship may also have a long-lasting effect and contribution to the sustainability of (re) integration long after the victim has exited assistance programmes. If these relationships are built on trust, the service providers remain a valuable resource for the survivor in the future, in case of need or difficult situations. The process of building these relationships is key and begins with a clear, open and honest relay of information from the service provider to the victim, without the expectation of reciprocity. This thesis has found that as time goes on, survivors that have built a stronger relationship with their service providers then maintain that relationship. In certain cases, the relationship takes the shape of occasional talks and the survivor herself checking in with the service provider and providing updates. One may argue that in these cases, such relationships may also satisfy the need of trafficked persons to still have 
someone to talk to that knows all their past experiences, particularly in cases where they have chosen not to share their past with their current families.

In other situations, however, this research has found that trafficked persons contact their service providers even many years after the trafficking experience and after they have exited the assistance programmes, to ask for help with problems they may currently face in their life. Problems may range from assistance needed in taking care of their children, to assistance they need in preventing their current friends or families from finding out about their past. This is consistent with literature on the recovery from sexual assault, which finds that when survivors find a therapist that is trustworthy, they may turn to that therapist many years after their experience, concerning other life issues as well (Starzynskia et al., 2017).

Regardless of what the reasons for sustaining the relationship with the service providers may be, it is clear that the service provider remaining available as a possible resource in the future, may be one way of preventing re-trafficking or even other types of exploitation, such as situations of domestic violence. A word of caution, however, there is always the possibility that the trafficked person would rely too heavily on the assistance and care from the service provider and this situation of loss of autonomy should be avoided.

\subsubsection{Prosecutions and trials (Chapter 6)}

Literature on recovery from rape and recovery of women from domestic violence have shown the value of criminal justice for the victim (CurtisFawley and Daly, 2005; Herman, 2003). However, in the context of trafficking- taking into consideration certain factors such as the longevity of the trial against the trafficker as well as the need of testimony from the trafficked person, which may trigger re-victimisation- the influence of the trial against the trafficker on the person trafficked cannot be seen clearly as either positive or negative. As already noted in the introductory chapter of this thesis, Chapter 6: Trafficking of Women for Sexual Exploitation in Europe: Prosecution, trials and their impact, is more exploratory in its nature. The influence of prosecutions and trials on the (re) integration of victims of trafficking for the purpose of sexual exploitation is a relatively new area of study. If one considers that legislation that would allow prosecution of traffickers for the crime of trafficking was not introduced more widely at the national level until the 2000 s, and if one additionally takes into consideration one of the main findings of this research that sometimes trials take many years, it is not unusual that little research exists on this topic. Thus, the 
contribution of this chapter is in beginning to explore this area of study and identifying certain occurrences in practice, which may influence (re) integration processes of victims.

As already noted, two of the main observations of this chapter have been that trials against traffickers may last for a very long time, in certain cases up to 10 years and more, and throughout this time period victims may be asked to testify multiple times, even many years after the trafficking experience ended. Such processes of remembering the details of the trafficking experience, many years after the experience itself may re-victimise the person. Thus, more research is needed to determine if such cases have any positive influence on the (re) integration process of the victim, and if it is the satisfied need for justice that dominates, or the damage done through the remembering process necessary to testify.

What is clearer, is that cases where trials have been swifter, have the ability to not only satisfy the need of the woman to see her abuser put away, but also make her feel safer in the years to come, since he or she will be incarcerated. In addition, another distinct conclusion of this chapter links back to the relationship with the service provider. It has been found that relaying information concerning the developments of the trial to the victim by the service provider throughout the process, is beneficial to her (re) integration. It also serves to build further trust between service provider and victim. Thus, once again, the value of open, clear, trustworthy information given to the victim, as it has been found in other research, comes to the forefront (Brunovskis \& Surtees, 2012; Walsh, Black \& Koser, 1999; Koser \& Pinkerton, 2002).

\subsubsection{Sex work, trafficking and stigma (Chapter 7)}

There may be clearly identifiable health reasons (mental and physical health) to explain why a person who has been trafficked should not return to sex work following a trafficking experience. It is an environment that places the victim in a vulnerable situation with many possible hazards and dangers (Saunders, 2005). However, while being exploited once again should the person return to sex work remains a significant risk, being stigmatised for being connected to sex work and the damages that such stigmatisation causes the (re) integration process, is a certainty.

The last chapter of this thesis focuses on how sex work is addressed in the context of (re) integration of victims of trafficking for the purpose of sexual 
exploitation. It was found that service providers often discourage trafficking victims from considering returning to sex work following a trafficking experience. This is in line with existing research (Brunovskis \& Surtees, 2008; Worthen, 2011). Sex work carries its own dangers and a person that was already exploited may be at higher risk for falling victim again. However, the perhaps more significant finding of this chapter is the ongoing stigmatisation of not only sex workers by both service providers and victims of trafficking, but also of trafficking victims. This is precisely because of the association of trafficking victims with the issue of sex work and because of the lack of understanding of the differences between sex work and sex trafficking. By analysing the reports from service providers and victims on this topic, the stigmatisation process was clearly traced (Goffman, 1963; Link $\&$ Phelan, 2001). The existence of public stigma, institutional stigma, as well as self-stigma was clearly visible (Bos et al., 2013). It was visible through the advice some service providers gave to victims to not expose their past to their employers (institutional stigma). It was visible through the terminology used to describe sex workers, through the way sex work was labelled as 'not normal work' and persons who choose this work as somehow 'not normal' (public stigma). And finally, it was visible through the attempts of all victims interviewed for this thesis trying to distance themselves from any conscious choice they may have made to enter sex work or justify the conscious choice they may have made to enter sex work prior to the trafficking.

The existence of stigma around the issue of sex work and consequently by association with the issue of trafficking for the purposes of sexual exploitation, has at least 2 clear consequences for the (re) integration process of trafficking victims. Firstly, existence of stigma influences the psychological, individual dimension of recovery of the trafficking victim. The occurrence that victims themselves self-stigmatised means that they may have a harder time to fully recover from the experience (Bos et al., 2013; Major \& O'Brien, 2005). As already noted, some victims may choose to share their experience with those closest to them, others may not. One of the reasons for not sharing their experience is that they may be re-victimised when speaking about it. However, another reason for not sharing their experiences may be the fear of being stigmatised. Thus, there may be women that wish to talk about their past, but because of fear of stigma, cannot, and this could possibly interfere with their ability to form healthy relationships.

Another consequence of stigma around these issues is the possible loss of trust between victim and service provider. In the context of recovery from 
sexual assault, survivors reported mistrust toward their therapist, when they felt that they were being blamed or judged for the occurrence of the exploitation (Campbell et al., 2001; Glass et al., 2000; Starzynskia et al., 2017). The mistrust led toward breaking of the relationship with the therapist, and in some cases, discontinuing treatment altogether, which of course had negative impact on the recovery process (Starzynskia et al., 2017). If victims fear being stigmatised, particularly in cases where they made the choice to enter sex work and were subsequently trafficked, they may never share their experience with service providers. If they plan to re-enter sex work following a trafficking experience, they may never share their plans with the service provider. Then it becomes irrelevant whether or not sex work is a possible choice for victims of trafficking as service providers, ignorant of the plans of the victim to re-enter sex work, may never get a chance to present their arguments in either direction. Overall, these situations would undermine the trust building process between trafficking victims and service providers. Moreover, not having the possibility to establish a close relationship with a service provider may have long-term consequences for (re) integration attempts. This would be particularly relevant in cases where trafficked persons do return to sex work and do fall into a situation of exploitation - they would not ask for help from a service provider out of fear of being judged or shamed.

To summarise, the above sections have attempted to present the main conclusions of each of the individual thesis chapters in a topical way: (1) recovery and (re) integration (2) autonomy versus independence (3) healthy relationships (4) prosecutions and trials and finally, (5) sex work, trafficking and stigma.

\subsection{Limitations and implications of the research}

\subsubsection{General}

The main strength of this research has been to draw on the experience of service providers and victims not only immediately after the trafficking experience but focusing on the (re) integration needs of the victims in the long run. Research up until now has placed a lot of focus on the needs of victims immediately after identification as trafficked - immediately meaning up to 3 years after the trafficking experience as 3 years is the length of monitoring and evaluation programs that offer assistance to victims in practice. However, what happens to victims after this monitoring period has mostly remained unexplored. This research is a step in that direction. 
It has also explored factors such as the effect of criminal prosecution and trials of traffickers on the (re) integration of victims, as well as the issue of stigmatisation of sex work and how that influences (re) integration processes of victims. How criminal proceedings influence recovery of rape victims has been researched, however, there are significant differences between these two groups of victims (rape victims and trafficked persons) when it comes to how they experience the criminal prosecution and trial of their abusers.

The main weakness of this research is that it began with such a wide scope of study, thus it may lack depth regarding certain more specific topics. Subsequently, it was found that the formation of new families has a significant influence on long-term (re) integration of trafficking victims. However, it could not go deeper to investigate the exact nature of these relationships, how were they formed, and how are they sustained. It was found in this research that victims often lose trust in friends and put a low value on friendships in general. However, this research falls short when it comes to investigating why this happens. Finally, although this research did find that stigma plays a significant role in how sex work is approached in post trafficking situations, it did not explore what happens to those women that nonetheless return to sex work following a trafficking experience.

\subsubsection{Methodological}

Often when looking at research on (re) integration after trafficking, the main goal is to reach victims and speak to them about their experiences. This research has managed to do that. However, the fact that service providers may be an incredibly rich source of information that gives insight into (re) integration processes is often overlooked. Throughout this research, service providers that have worked directly with victims of trafficking, in some cases for many years, were consulted. They were not only able to speak on how the needs of the victim changed through time, but also able to compare the experiences of one beneficiary with another in the short and long-term.

Victims of trafficking are unlikely to be fully accessible as a group as they may be considered a 'hidden population'. (Tyldum \& Brunovskis, 2005). Some of them, once leaving assistance programs, break all ties with service providers, in particular due to issues of stigma, which makes them impossible to locate (Heckathorn, 1997 as quoted in Tyldum \& Brunovskis, 2005). In addition, as Chapter 6: Trafficking of Women for Sexual Exploitation in Europe showed, interviewing victims of trafficking should be approached with great caution. For many, discussing the trafficking experience may mean 
re-living it again, and thus re-victimisation. Due to ethical reasons, researchers should consider if such interviews should be conducted at all. And in the absence of those interviews, a remaining valuable source of information is the service providers with first-hand experience and knowledge.

This particular thesis tried to strike a balance in this regard - it was decided that attempts should be made to reach some victims of trafficking. Due to issues of access (discussed in detail in Chapter 3) and due to ethical considerations, this number has remained small. Although some may consider this to be a weakness of this research, it can also be seen as a strength, as it has led to greater focus on feedback from service providers, who in particular were able to shed light on (re) integration processes in the long run.

Finally, this research attempted to use a methodology that is relatively unexplored in this field - personal diaries that would be kept by victims over a longer period of time. The use of such diaries would possibly have provided a wealth of information on the current state of being of each of the victims that were interviewed. In addition, as writing is often used as a therapeutic method, it could have had positive influences for the (re) integration of the victim as well. However, although 2 victims interviewed for this study agreed to keep such diaries, they were unavailable when the researcher attempted to re-establish contact one month after they began writing. Thus, keeping in touch with victims in these cases was a methodological challenge that potentially prevented valuable material from being used in this research.

\subsection{Implications: theoretical and practical}

Theoretically and practically, what has been highlighted during (re) integration efforts of victims of trafficking for the purpose of sexual exploitation, is the importance of 'independence' of the victim.

A lot of emphasis is already placed on women that have been victims of trafficking being economically empowered and being financially independent. This is not necessarily the wrong focus of assistance programmes, although the very nature of the assistance programmes offered in these situations and how much their content is truly of help in economically empowering women could be questioned. One finds almost always, the same types of training mentioned across all assistance organisations - computer lessons, language lessons, cooking, dress making, and hairdressing classes. Are these really effective? A question that was not answered in this thesis, 
but should be by objective researchers and not by evaluation reports written by those financing or implementing these types of programmes. Also, and rightly so, a lot of emphasis in assistance programmes is placed on women, where possible, reconciling with their families of origin or with families they had formed prior to the trafficking experience.

However, what seems to be missing is any emphasis placed on making sure that new relations formed by trafficking victims following a trafficking experience, are healthy ones, and that the victim re-establishes the ability to start new relationships as well. The danger of victims of trafficking entering relationships that may place them back in a situation of exploitation, precisely because of their previous exploitative experiences, is particularly high, as illustrated in the example later in this section. On the other hand, the benefit of forming new, healthy relationships following a trafficking experience contributes to making sustainable progress regarding their (re) integration. What this thesis found is that the ability to form healthy relationships was mainly addressed through therapy sessions that victims underwent. Still, it is questionable if this is enough. To give one example from this research, which was covered in Chapter 5, a beneficiary was returned to her family when it was discovered that she was again making ties with persons that may exploit her. In this case, she had a supportive family that prevented any abuse from occurring. However, what happens to those women who do not have the support of their families? If organisations have means and programs that would address these needs of victims - to restore their ability to form new relationships that are healthy and not harmful, then there would be no need for families of origin, which are sometimes absent or not an option, to intervene.

Discussing the precise nature of such programmes would be beyond the scope of this research and their design should perhaps be constructed with the help of professionals from the field of psychology. Nonetheless, the need of such programmes is evident. The same could be said of programmes that would further train service providers in establishing relationships based on trust with their beneficiaries.

Chapter 6: Trafficking of Women for Sexual Exploitation in Europe: Prosecution, trials and their impact, gave precise recommendations regarding how criminal prosecutions and trials of the traffickers should be handled in the context of (re) integration of the victim. The main point to be emphasised is that in certain countries, in particular origin countries of Southeastern 
Europe, prosecutions and trials simply last too long, in some cases up to 10 years. This should be unacceptable. Mechanisms should be put in place that would ensure the flow of the prosecution and trial procedures, regardless of administrative changes within the justice system. Another point that should be highlighted is that asking a victim to testify in court against her trafficker already creates the danger that re-victimisation could occur. There should be ways to overcome this and policies in place, such as video testimonies, that would at least ensure she does not come in contact with her abuser. It should be enough for victims to testify once, and policies are needed that would make it possible to use this same testimony even many years after it was given. Contacting a victim to testify again, in some cases many years after her trafficking experience, is not only harmful to her health, but also possibly inaccurate, as much time has passed from the trafficking experience itself.

Finally, Chapter 7: Sex work and trafficking for the purpose of sexual exploitation showed the damaging effect stigmatisation of sex work and by extension, trafficking victims can have on their (re) integration. The debate as to whether sex work can be a choice is ongoing. The view of most service providers working with victims of trafficking is that they should not return to sex work following a trafficking experience. However, we do not have to wait for all the debate around the issues of sex work to be resolved in order to emphasise that the way we speak about sex work is damaging to the women involved and affected by it. Efforts should be made to end stigma around the topic and they can begin by choosing to use the term 'sex work' and not 'prostitution', as this research has done, following the results of Chapter 7.

\subsection{Recommendations for future research}

Significant questions were raised throughout the research for this thesis, but due to its wide scope, this particular work could not go deeper and attempt to answer them.

Primarily, there is a need for future research to investigate the dynamics of interactions between survivor and her new family formed following a trafficking experience. In particular what should be looked at are success cases. Looking at the particular personality traits, their backgrounds, and any help and assistance they may have gotten along the way of women that have formed such healthy ties, could be indicative of the precise policies that could be introduced that would help other victims of trafficking in regaining the ability to form healthy relationships. Looking at the interactions between 
family members may also be useful, as well as how, if in any way, the former trafficking experience is being addressed. Of course, there are ethical issues to be considered here, as there needs to be clear willingness from the survivor and possibly, where the family is aware of her past, the family members in order to conduct such research.

As already noted, this thesis showed that many of the victims had lost trust in past and current friendships and seemed to not establish or re-establish such relationships. There needs to be more research into the reasons as to why this is the case.

Finally, this research was not able to reach women that have been trafficked and thereafter returned to sex work. (Re) integration efforts of this group could arguably significantly differ from those women that do not go back to sex work, and consequently their needs may be very different. The primary purpose should always be ensuring that a woman who was a victim of trafficking once, would not become so again, and would not find herself again in a situation where she is being abused or exploited. At the moment, this group of women is stigmatised, and as a result of that, seems to have no public voice at all. 


\section{References:}

Abas, M. (2012). Physical health symptoms reported by trafficked women receiving post-trafficking support in Moldova: Prevalence, severity and associated factors. BMC Women's Health, 12, 1-9.

doi: 10.1186/1472-6874-12-20

Abas, M., Ostrovschi, N. V., Prince, M., Gorceag, V. I., Trigub, C., \& Oram, S. (2013). Risk factors for mental disorders in women survivors of human trafficking: A historical cohort study. BMC Psychiatry, 13, 1-11. doi: 10.1186/1471-244X-13-204

Acharya, A. K. (2010). Feminization of migration and trafficking of women in Mexico. Revista de Cercetare şi Intervenţie Socială, 30, 19-38.

Agustín, L. (2006). The disappearing of a migration category: Migrants who sell sex. Journal of Ethnic and Migration Studies, 32, 29-47. doi:

10.1080/13691830500335325

Allred, K. J. (2006). Peacekeepers and prostitutes how deployed forces fuel the demand for trafficked women and new hope for stopping it. Armed Forces \& Society, 33, 5-23. doi: 10.1177/0095327X06288803

Amar, P. (2009). Operation princess in Rio de Janeiro: Policing 'sex trafficking', strengthening worker citizenship, and the urban geopolitics of security in Brazil. Security Dialogue, 40, 513-541. doi:

10.1177/0967010609343300

Anderson, B., \& Andrijasevic, R. (2008). Sex, slaves and citizens: The politics of anti-trafficking. Soundings, 40, 135-145.

Anderson, B., \& O'Connell Davidson, J. (2003). The demand side of trafficking. A multi-country study. Stockholm: Save the Children.

Andrijasevic, R. (2003). The difference borders make: (Il)legality, migration and trafficking in Italy among Eastern European women in prostitution. In: S. Ahmed, C,. Castaneda, A. Fortier, \& M. Sheller (Eds.), Uprootings/regroundings: Questions of home and migration (pp. 251-272). New York: Berg. 
Andrijasevic, R. (2007). Beautiful dead bodies: Gender, migration and representation in anti-trafficking campaigns. Feminist Review, 86, 24-44. doi: $10.1057 /$ palgrave.fr.9400355

Andrijasevic, R., Aradau, C., Huysmans, J., \& Squire, V. (2012). European citizenship unbound: sex work, mobility, mobilisation. Environment and Planning D: Society and Space, 30, 497-514.

Aradau, C. (2004). The perverse politics of four-letter words: Risk and pity in the securitisation of human trafficking. Millennium Journal of International Studies, 33, 251-277. doi: 10.1177/03058298040330020101

Aronowitz, A. A. (2001). Smuggling and trafficking in human beings: The phenomenon, the markets that drive it and the organisations that promote it. European Journal on Criminal Policy and Research, 9, 163-195.

Asquith, R., Turner, E. (2008) (Re) integration of Children from the Effects of Sexual Exploitation and Related Trafficking. Oak Foundation Child Abuse Programme, Geneva.

Bales, K. (2012). Disposable people: New slavery in the global economy. Berkeley, CA: California University Press.

Barnhart, M. H. (2009). Sex and slavery: An analysis of three models of state human trafficking legislation. William \& Mary Journal of Women and the Law, 16, 83-131.

Bartholomew, L. K., Parcel, G. S., Kok, G., Gottlieb, N. H., \& Fernández, M. E. (2011). Planning health promotion programs: An intervention mapping approach (3rd ed.). San Francisco, CA: Jossey Bass.

Baumeister, R., \& Leary, M. (1995). The need to belong : Desire for interpersonal attachments as a fundamental human motivation. Psychological Bulletin, Vol. 117 No. 3 (mayo 1995).

Bearup, L.S. (2015) 'Trafficking and Protection: Theorising Reintegration and Defining Success'. Doctoral Thesis. Deaking University. 
Bennett, L., \& O-Brien, P. (2007). Effects of coordinated services for drugAbusing women who are victims of intimate partner violence. Violence against Women: An International and Interdisciplinary Journal, 13(4), 395411.

Berman, J. (2003). (Un) Popular strangers and crises (un)bounded:

Discourses of sex trafficking, the European political community and the panicked state of the modern state. European Journal of International Relations, 9, 37-86. doi: 10.1177/1354066103009001157

Bernstein, E. (2007). The sexual politics of the "new abolitionism". Differences, 18, 128-151. doi: 10.1215/10407391-2007-013

Billette, V., Guay, S., \& Marchand, A. (2008). Posttraumatic stress disorder and social support in female victims of sexual assault: The impact of spousal involvement on the efficacy of cognitive-behavioral therapy. Behavior Modification, 32(6), 876-96. doi:10.1177/0145445508319280

Bjerkan, L. (2005). A life of one's own: Rehabilitation of victims of trafficking for sexual exploitation. Norway: Allkopi AS.

Black, R., \& Koser, K. (1998). The end of the refugee cycle? : Refugee repatriation and reconstruction (Refugee and forced migration studies), v. 4). New York: Berghahn Books.

Bletzer, K., \& Koss, M. (2006). After-Rape among three populations in the southwest: A time of mourning, a time for recovery. Violence against Women, 12(1), 5-29.

Bonthuys, E. (2012). The 2010 football world cup and the regulation of sex work in South Africa. Journal of Southern African Studies, 38, 11-29. doi: $10.1080 / 03057070.2012 .642723$

Bos, A. E. R., Schaalma, H. P., \& Pryor, J. B. (2008). Reducing AIDSrelated stigma in developing countries: The importance of theory- and evidence- based interventions. Psychology, Health \& Medicine, 13, 450460. doi: 10.1080/13548500701687171 
Bos, A. E. R., Pryor, J. B., Reeder, G. D., \& Stutterheim, S. E. (2013).

Stigma: Advances in theory and research. Basic and Applied Social Psychology, 35, 1-9. doi: 10.1080/01973533.2012.746147

Bownes, I.T. O'Gorman, E.C. \& Sayers, A. (1991). Assault characteristics and posttraumatic stress disorder in rape victims. Acta Psychiatrica Scandinavica, 83(1), 27-30.

Brennan, D. (2014). Trafficking, scandal, and abuse of migrant workers in Argentina and the United States. The ANNALS of the American Academy of Political and Social Science, 653, 107-123. doi:10.1177/0002716213519239

Bronfenbrenner, U. (1979). The ecology of human development : Experiments by nature and design. Cambridge, Mass.: Harvard University Press.

Brown, N., Kallivayalil, D., Mendelsohn, M., \& Harvey, M. (2012). Working the double edge: Unbraiding pathology and resiliency in the narratives of early-recovery trauma survivors. Psychological Trauma: Theory, Research, Practice, and Policy, 4(1), 102-111. doi:10.1037/a0024969

Brunovskis, A., \& Surtees, R. (2008). Agency or illness: The conceptualization of trafficking victims' choices and behaviors in the assistance system. Gender, Technology and Development, 12, 53-76. doi: 10.1177/097185240701200105

Brunovskis, A., \& Surtees, R. (2012a). Out of sight? Approaches and challenges in the identification of trafficked persons. Norway: Allkopi AS.

Brunovskis, A., Surtees, R., (2012b). Coming home: Challenges in family reintegration for trafficked women. Qualitative Social Work, 12, 454-472. doi: $10.1177 / 1473325011435257$

Burgess, A., \& Holmstrom, L. (1978). Recovery from rape and prior life stress. Research in Nursing \& Health, 1(4), 165-174.

doi:10.1002/nur.4770010404 
Burgess, A., \& Holmstrom, L. (1979). Rape: Sexual Disruption and Recovery. American Journal of Orthopsychiatry, 49(4), 648-657. doi:10.1111/j.1939-0025.1979.tb02650.x

Burt, M., \& Katz, B. (1987). Dimensions of recovery from rape - focus on growth outcomes. Journal of Interpersonal Violence, 2(1), 57-81.

Burt, M., \& Katz, B. (1988). Coping strategies and recovery from rape. Annals of the New York Academy of Sciences, 528(1), 345-358. doi:10.1111/j.1749-6632.1988.tb50876.x

Busch-Armendariz, N., Nsonwu, M. B., \& Heffron, L. C. (2014). A kaleidoscope: The role of the social work practitioner and the strength of social work theories and practice in meeting the complex needs of people trafficked and the professionals that work with them. International Social Work, 57, 7-18. doi: 10.1177/0020872813505630

Campbell, R. Dworkin, E. \& Cabral, G. (2009). An ecological model of the impact of sexual assault on women's mental health. Trauma, Violence \& Abuse, 10(3), 225-46. doi:10.1177/1524838009334456

Campbell, R., Wasco, M.S., Ahrens, E.C., Sefl, T., \& Barnes, E.H. (2001). Preventing the "second rape" : Rape survivors' experiences with community service providers. Journal of Interpersonal Violence, 16(12), 1239-1259. doi:10.1177/088626001016012002

Chapkis, W. (2003). Trafficking, migration, and the law protecting innocents, punishing immigrants. Gender \& Society, 17, 923-937. doi: $10.1177 / 0891243203257477$

Chin, K. L., \& Finckenauer, J. O. (2011). Chickenheads, agents, mommies, and jockeys: The social organization of transnational commercial sex. Crime, Law and Social Change, 56, 463-484. doi: 10.1007/s10611-0119329-y

Cho, S. Y. (2013). Integrating equality: Globalization, women's rights, and human trafficking. International Studies Quarterly, 57, 683-697. doi: 10.1111 isqu. 12056 
Cho, S. (2016). Liberal coercion? prostitution, human trafficking and policy. European Journal of Law and Economics, 41(2), 321-348.

doi:10.1007/s10657-015-9519-7

Cho, S. Y., Dreher, A., \& Neumayer, E. (2013). Does legalized prostitution increase human trafficking? World Development, 41, 67-82.

doi: 10.1016/j.worlddev.2012.05.023

Chudakov, B., Ilan, K., Belmaker, R. H., \& Cwikel, J. (2002). The motivation and mental health of sex workers. Journal of Sex \& Marital Therapy, 28, 305-315. doi: 10.1080/00926230290001439

Cohen, L., \& Roth, S. (1987). The psychological aftermath of rape: LongTerm effects and individual differences in recovery. Journal of Social and Clinical Psychology, 5(4), 525-534.

Collins, S. P., Goldenberg, S. M., Burke, N. J., Bojorquez-Chapela, I., Silverman, J. G., \& Strathdee, S. A. (2013). Situating HIV risk in the lives of formerly trafficked female sex workers on the Mexico-US border. AIDS Care, 25, 459-465. doi: 10.1080/09540121.2012.720361

Copley, L. (2014). Neutralizing their involvement sex traffickers' discourse techniques. Feminist Criminology, 9, 45-58.

doi: $10.1177 / 1557085113501849$

Crawford, M., \& Kaufman, M. R. (2008). Sex trafficking in Nepal survivor characteristics and long-term outcomes. Violence Against Women, 14, 905916. doi: 10.1177/1077801208320906

Cribb, J., \& Barnett, R. (1999). Being bashed : Western samoan women's responses to domestic violence in Western Samoa and New Zealand. Gender, Place and Culture (print), 6(1999):1, S. 49-65.

Criminal Code of the Netherlands (Wetboek van Strafrecht), Section 36f, Subsection (6).

Curtis-Fawley, S., \& Daly, K. (2005). Gendered violence and restorative justice: The views of victim advocates. Violence against Women, 11(5), 603-638. 
Dahal, P Joshi, S.K. \& Swahnberg, K. (2015). 'We are looked down upon and rejected socially': A qualitative study on the experiences of trafficking survivors in Nepal. Global Health Action, 8, 29267-29267.

doi:10.3402/gha.v8.29267

Decker, M. (2013). Sex trafficking, sex work, and violence: Evidence for a new era. International Journal of Gynecology and Obstetrics, 120(2), 113114. doi:10.1016/j.ijgo.2012.11.001

Decker, M. R., McCauley, H. L., Phuengsamran, D., Janyam, S., \& Silverman, J. G. (2011). Sex trafficking, sexual risk, sexually transmitted infection and reproductive health among female sex workers in Thailand. Journal of Epidemiology and Community Health, 65, 334-339. doi: 10.1136/jech.2009.096834

Deering, K. N., Chettiar, J., Chan, K., Taylor, M., Montaner, J. S., \& Shannon, K. (2012). Sex work and the public health impacts of the 2010 Olympic games. Sexually Transmitted Infections, 88, 301-303. doi: 10.1136/sextrans-2011-050235

Dempsey, M. M. (2010). Sex trafficking and criminalization: In defense of feminist abolitionism. University of Pennsylvania Law Review, 158, 17291778 .

Deshpande, N. A., \& Nour, N. M. (2013). Sex trafficking of women and girls. Reviews in Obstetrics and Gynecology, 6, e22.

Di Tommaso, M. L., Shima, I., Strøm, S., \& Bettio, F. (2009). As bad as it gets: Wellbeing deprivation of sexually exploited trafficked women.

European Journal of Political Economy, 25, 143-162.

doi: 10.1016/j.ejpoleco.2008.11.002

Ditmore, M. (2011). Prostitution and sex work (Historical guides to controversial issues in america). Santa Barbara, Calif.: Greenwood.

Doezema, J. (1999). Loose women or lost women? The re-emergence of the myth of white slavery in contemporary discourses of trafficking in women. Gender Issues, 18, 23-50. doi: 10.1007/s12147-999-0021-9 
Doezema, J. (2005). Now you see her, now you don't: Sex workers at the UN trafficking protocol negotiation. Social \& Legal Studies, 14, 61-89. doi: $10.1177 / 0964663905049526$

Doezema, J. (2010). Sex slaves and discourse masters: The construction of trafficking. London: Zed Books.

Ellis, E. (1983). A review of empirical rape research: Victim reactions and response to treatment. Clinical Psychology Review, 3(4), 473-490.

doi:10.1016/0272-7358(83)90025-9

Farrell, A. Owens, C. \& McDevitt, J. (2014). New laws but few cases: Understanding the challenges to the investigation and prosecution of human trafficking cases. Crime, Law and Social Change, 61(2), 139-168. doi:10.1007/s10611-013-9442-1

Few, A. (2005). The voices of black and white rural battered women in domestic violence shelters. Family Relations, 54(4), 488-500.

Fischbach, R., \& Herbert, B. (1997). Domestic violence and mental health : Correlates and conundrums within and across cultures. Social Science \& Medicine (print), 45(1997):8, S. 1161-1176.

Fitzpatrick, J. (2002). Trafficking and a human rights violation: The complex intersection of legal frameworks for conceptualizing and combating trafficking. Michigan Journal of International Law, 24, 1143.

Flick, U. (2009). An introduction to qualitative research (4th ed. ed.). Los Angeles: Sage Publications.

Frazier, P., \& Burnett, J. (1994). Immediate coping strategies among rape victims. Journal of Counseling \& Development, 72(6), 633-639. doi:10.1002/j.1556-6676.1994.tb01694.x

Frazier, P., \& Schauben, L. (1994). Causal attributions and recovery from rape and other stressful life events. Journal of Social \& Clinical Psychology, Vol. 13, No. 1 (1994), P. 1-14. 
Gallagher, A. (2001). Human rights and the new UN protocols on trafficking and migrant smuggling: A preliminary analysis. Human Rights Quarterly, 23, 975-1004. doi: 10.1353/hrq.2001.0049

Gallagher, A. T. (2010). The international law of human trafficking. Cambridge: Cambridge University Press.

Gan, C., Ha L.T.T., Kao, B., Poch, K. (2014) An Assessment of the Role of Nongovernment Organisations in Combating Trafficking of Women and Children in Cambodia and Viet Nam Journal of GMS Development Studies. 6. 71-91.

Gjermeni, E., Van Hook, M., Gjipali, S., Xhillari, L., Lungu, F., \& Hazizi, A. (2008). Trafficking of children in albania: Patterns of recruitment and reintegration. Child Abuse \& Neglect: The International Journal, 32(10), 941-948.

Glass, C., \& Arnkoff, D. (2000). Consumers' perspectives on helpful and hindering factors in mental health treatment. Journal of Clinical Psychology, 56(11), 1467-1480. doi:10.1002/10974679(200011)56:113.0.CO;2-K

Goffman, E. (1968). Stigma : Notes on the management of spoiled identity (Pelican books). Harmondsworth: Penguin Books.

Goodey, J. (2003). Migration, crime and victimhood responses to sex trafficking in the EU. Punishment \& Society, 5, 415-431. doi: $10.1177 / 14624745030054003$

Goodey, J. (2004). Sex trafficking in women from central and east european countries : Promoting a "victim-centred" and "woman-centred" approach to criminal justice intervention. Feminist Review (print), 2004, 76, S. 26-45.

Gould, C. (2010). Moral panic, human trafficking and the 2010 soccer world cup. Agenda, 24, 31-44. doi: 10.1080/10130950.2010.9676321

Harvey, M. (1996). An ecological view of psychological trauma and trauma recovery. Journal of Traumatic Stress, 9(1), 3-23.

doi:10.1002/jts.2490090103 
Harvey, M. (2007). Towards an ecological understanding of resilience in trauma survivors: Implications for theory, research, and practice. Journal of Aggression, Maltreatment \& Trauma, 14(1/2), 9-32.

Haynes, D. F. (2004). Used, abused, arrested and deported: Extending immigration benefits to protect the victims of trafficking and to secure the prosecution of traffickers. Human Rights Quarterly, 26, 221-272.

Helfrich, C., \& Aviles, A. (2001). Occupational therapy's role with victims of domestic violence: Assessment and intervention. Occupational Therapy in Mental Health, 16, 53-70.

Herman, J. (2003). The mental health of crime victims impact of legal intervention. Journal of Traumatic Stress, 16(2), 159-166.

Hill, J., \& Zautra, A. (1989). Self-Blame attributions and unique vulnerability as predictors of post-Rape demoralization. Journal of Social and Clinical Psychology, 8(4), 368-375.

Hom, K. A., \& Woods, S. J. (2013). Trauma and its aftermath for commercially sexually exploited women as told by front-line service providers. Issues in Mental Health Nursing, 34, 75-81. doi: 10.3109/01612840.2012.723300

Houte, M., Driessen, K., Koser, K., \& Maastricht Graduate School of Governance. (2014). Moving back or moving forward? : Return migration after conflict (Doctoral dissertation, 2014). Uitgever niet vastgesteld.

Hovell, M., Seid, A., \& Liles, S. (2006). Evaluation of a police and social services domestic violence program: Empirical evidence needed to inform public health policies. Violence against Women, 12(2), 137-159.

Hoyle, C., Bosworth, M., \& Dempsey, M. (2011). Labelling the victims of sex trafficking: Exploring the borderland between rhetoric and reality. Social \& Legal Studies, 20, 313-329. doi: 10.1177/0964663911405394

Hua, J., \& Nigorizawa, H. (2010). US sex trafficking, women's human rights and the politics of representation. International Feminist Journal of Politics, 12, 401-423. doi:10.1080/14616742.2010.513109 
Hubbard, P., Matthews, R., \& Scoular, J. (2008). Regulating sex work in the EU: Prostitute women and the new spaces of exclusion. Gender, Place and Culture, 15, 137-152. doi: 10.1080/09663690701863232

Hughes, D. M. (2000). The "Natasha" trade: The transnational shadow market of trafficking in women. Journal of International Affairs-Columbia University, 53, 625-652.

Huisman, W., \& Kleemans, E. R. (2014). The challenges of fighting sex trafficking in the legalized prostitution market of the Netherlands. Crime, Law and Social Change, 61, 1-14. doi: 10.1007/s10611-013-9512-4

Immordino, G., \& Russo, F. (2015a). Regulating prostitution: A health risk approach. Journal of Public Economics, 121, 14-31.

doi:10.1016/j.jpubeco.2014.11.001

Immordino, G., \& Russo, F. (2015b). Laws and stigma: The case of prostitution. European Journal of Law and Economics, 40(2), 209-223. doi:10.1007/s10657-015-9491-2

ILO (2012). Global Estimate of Forced Labour 2012: Results and Methodology. Geneva: International Labor Organization.

Jakobsson, N., \& Kotsadam, A. (2011). Gender equity and prostitution: An investigation of attitudes in Norway and Sweden. Feminist Economics, $17(1)$.

Jakobsson, N., \& Kotsadam, A. (2013). The law and economics of international sex slavery: Prostitution laws and trafficking for sexual exploitation. European Journal of Law and Economics, 35, 87-107. doi: 10.1007/s10657-011-9232-0

Jayagupta, R. (2009). The Thai Government's repatriation and reintegration programmes: Responding to trafficked female commercial sex workers from the Greater Mekong Subregion. International Migration, 47, 227-253. doi: $10.1111 /$ j.1468-2435.2008.00498.x

Jeffreys, S. (2009). Prostitution, trafficking and feminism: An update on the debate. Women's Studies International Forum, 32, 316-320.

doi: 10.1016/j.wsif.2009.07.002 
Jones, L., Engstrom, D., Hilliard, P., \& Sungakawan, D. (2011). Human trafficking between Thailand and Japan: Lessons in recruitment, transit and control. International Journal of Social Welfare, 20, 203-211. doi: 10.1111/j.1468-2397.2009.00669.x

Jongsma, A., Jr.,. (2010). Handbook of social psychology (5th ed. ed.) (S. Fiske, D. Gilbert, \& G. Lindzey, Eds.). Hoboken, N.J.: Wiley.

Jonsson, S., \& Jakobsson, N. (2017). Is buying sex morally wrong? comparing attitudes toward prostitution using individual-level data across eight western european countries. Women's Studies International Forum, 61(2), 58-69. doi:10.1016/j.wsif.2016.12.007

Jordan, A. D. (2002). Human rights or wrongs? The struggle for a rightsbased response to trafficking in human beings. Gender \& Development, 10, 28-37. doi: 10.1080/13552070215891

Jordan, C.E. (2009). Advancing the study of violence against women: Evolving research agendas into science. Violence against Women, 15(4), 393-419. doi:10.1177/1077801208330692

Kelland, L. (2012). A narrative model of recovery. South African Journal of Philosophy, 31(2), 290-300.

Kelly, L. (2003). The wrong debate: Reflections on why force is not the key issue with respect to trafficking in women for sexual exploitation. Feminist Review, 73, 139-144. doi: 10.1057/palgrave.fr.9400086

Kenyon, S., \& Schanz, Y. (2014). Sex trafficking: Examining links to prostitution and the routine activity theory. International Journal of Criminology and Sociology, 3, 61-76. Retrieved from https://www.ncjrs.gov/App/Publications/abstract.aspx?ID=267352

Kilpatrick, D., Resick, P., \& Veronen, L. (1981). Effects of a rape experience: A longitudinal study. Journal of Social Issues, 37(4), 105-122. doi:10.1111/j.1540-4560.1981.tb01073.

Kim, K., \& Hreshchyshyn, K. (2004). Human trafficking private right of action: Civil rights for trafficked persons in the United States. Hastings Women's Law Journal, 16, 1-36. 
Konstantopoulos, W. M., Ahn, R., Alpert, E. J., Cafferty, E., McGahan, A., Williams, T. P., ... \& Burke, T. F. (2013). An international comparative public health analysis of sex trafficking of women and girls in eight cities: Achieving a more effective health sector response. Journal of Urban Health, 90, 1194-1204. doi:10.1007/s11524-013-9837-4

Koser, K., Pinkerton, C., \& Great Britain. Home Office. Research, Development and Statistics Directorate. (2002). The social networks of asylum seekers and the dissemination of information about countries of asylum. London: Home Office, Research, Development and Statistics Directorate.

Koser, K., \& Kuschminder, K. (2015). Comparative Research on the Assisted Voluntary Return and Reintegration of Migrants. Geneva: International Organisation for Migration.

Koss, M., Herrera, V., \& Lichter, E. (2003). Depression and ptsd in survivors of male violence: Research and training initiatives to facilitate recovery. Psychology of Women Quarterly, 27(2), 130-142.

Le, P.D. (2017). "Reconstructing a sense of self": Trauma and coping among returned women survivors of human trafficking in Vietnam. Qualitative Health Research, 27(4), 509-519. doi:10.1177/1049732316646157

Leman, J., \& Janssens, S. (2013). Creative adaptive criminal entrepreneurs from Africa and human trafficking in Belgium: Case studies of traffickers from Nigeria and Morocco. International Journal of Criminology and Sociology, 2, 153-162. doi: 10.6000/1929-4409.2013.02.15

Limoncelli, S. A. (2009). The trouble with trafficking: Conceptualizing women's sexual labor and economic human rights. Women's Studies International Forum, 32, 261-269. doi: 10.1016/j.wsif.2009.05.002

Lindstrom, N. (2004). Regional sex trafficking in the Balkans: Transnational networks in an enlarged Europe. Problems of PostCommunism, 51, 45-52.

Link, B., \& Phelan, J. (2001). Conceptualizing stigma. Annual Review of Sociology, 27(1), 363-385. doi:10.1146/annurev.soc.27.1.363 
Littleton, H., \& Breitkopf, C. (2006). Coping with the experience of Rape. Psychology of Women Quarterly, 30(1), 106-116.

Lobasz, J. K. (2009). Beyond border security: Feminist approaches to human trafficking. Security Studies, 18, 319-344.

doi: 10.1080/09636410902900020

Lyneham, S., \& Larsen, J. J. (2013). Exploitation of Indonesian trafficked men, women and children and implications for support. Trends and Issues in Crime and Criminal Justice, 450, 1-7.

Mai, N. (2013). Embodied cosmopolitanisms: The subjective mobility of migrants working in the global sex industry. Gender, Place \& Culture, 20, 107-124. doi: 10.1080/0966369X.2011.649350

Major, B. \& O'Brien, L.T. (2005). The social psychology of stigma. Annual Review of Psychology, 56, 393-421.

Mancuso, M. (2014). Not all madams have a central role: Analysis of a Nigerian sex trafficking network. Trends in Organized Crime, 17, 66-88. doi: $10.1007 / \mathrm{s} 12117-013-9199-\mathrm{Z}$

Marcus, A., Horning, A., Curtis, R., Sanson, J., \& Thompson, E. (2014). Conflict and agency among sex workers and pimps a closer look at domestic minor sex trafficking. The ANNALS of the American Academy of Political and Social Science, 653, 225-246. doi: 10.1177/0002716214521993

Marinova, N. K., \& James, P. (2012). The tragedy of human trafficking: Competing theories and European evidence. Foreign Policy Analysis, 8 , 231-253. doi: 10.1111/j.1743-8594.2011.00162.x

Masten, A.S. (2007). Resilience in developing systems: Progress and promise as the fourth wave rises. Development and Psychopathology, 19(3), 921-30.

McIntyre, B. L. (2014). More than just rescue: Thinking beyond exploitation to creating assessment strategies for child survivors of commercial sexual exploitation. International Social Work, 57, 39-63. doi: $10.1177 / 0020872813505629$ 
Miriam, K. (2005). Stopping the traffic in women: Power, agency and abolition in feminist debates over sex-trafficking. Journal of Social Philosophy, 36, 1-17.doi: 10.1111/j.1467-9833.2005.00254.x

Molland, S. (2010). The value of bodies: Deception, helping and profiteering in human trafficking along the Thai-Lao border. Asian Studies Review, 34, 211-229. doi: 10.1080/10357823.2010.481042

Molland, S. (2011). The trafficking of scarce elite commodities: Social change and commodification of virginity along the Mekong. The Asia Pacific Journal of Anthropology, 12, 129-145.

doi: $10.1080 / 14442213.2010 .546426$

Muftić, L. R. (2013). Attitudes regarding criminal justice responses to sex trafficking among law enforcement officers in Bosnia and Herzegovina. Journal of Criminal Justice and Security, 2, 177-189.

Munro, V. E. (2006). Stopping traffic? A comparative study of responses to the trafficking in women for prostitution. British Journal of Criminology, 46, 318-333. doi: 10.1093/bjc/azi070

Munro, V. E. (2008). Of rights and rhetoric: Discourses of degradation and exploitation in the context of sex trafficking. Journal of Law and Society, 35, 240-264. doi: 10.1111/j.1467-6478.2008.00437.x

Neville, H., \& Heppner, M. (1999). Contextualizing rape: Reviewing sequelae and proposing a culturally inclusive ecological model of sexual assault recovery. Applied and Preventive Psychology, 8(1), 41-62. doi:10.1016/S0962-1849(99)80010-9

Nieuwenhuys, C., \& Pécoud, A. (2007). Human trafficking, information campaigns, and strategies of migration control. American Behavioral Scientist, 50, 1674-1695. doi: 10.1177/0002764207302474

O'Connor, M., \& Healy, G. (2006). The links between prostitution and sex trafficking: A briefing handbook. Dublin: Coalition Against Trafficking in Women.

O'Brien, E., Carpenter, B., \& Hayes, S. (2013). Sex trafficking and moral harm: Politicised understandings and depictions of the trafficked experience. Critical Criminology, 21, 401-415. doi: 10.1007/s10612-013-9183-6 
O’Connell Davidson, J. (2006). Will the real sex slave please stand up? Feminist Review, 83, 4-22.

O'Connell Davidson, J. (2013). Troubling freedom: Migration, debt, and modern slavery. Migration Studies, 1, 176-195.

O'Connell Davidson, J., \& Anderson, B. (2003). Is trafficking in human beings demand driven? A multi-country pilot study. Geneva: International Organization for migration

Oram, S., Ostrovschi, N. V., Gorceag, V. I., Hotineanu, M. A., Gorceag, L., Trigub, C., \& Outshoorn, J. (2012). Policy change in prostitution in the Netherlands: From legalization to strict control. Sexuality Research and Social Policy, 9, 233-243. doi: 10.1007/s13178-012-0088-z

Outshoorn, J. (2005). The political debates on prostitution and trafficking of women. Social Politics: International Studies in Gender, State \& Society, 12(1), 141-155.

Overs, C., \& Hawkins, K. (2011). Can rights stop the wrongs? Exploring the connections between framings of sex workers' rights and sexual and reproductive health. BMC International Health and Human Rights, 11, S6. doi: 10.1186/1472-698X-11-S3-S6

Pajnik, M. (2013). Reconciling paradigms of prostitution through narration. Društvena Istraživanja, 22, 257-276. doi: 10.5559/di.22.2.03

Pandey, S., Tewari, H. R., \& Bhowmick, P. K. (2013). Antecedents and reintegration of sex trafficked victims in India: A conceptual framework. International Journal of Criminal Justice Sciences, 8, 47-62.

Pickering, S., \& Ham, J. (2014). Hot pants at the border sorting sex work from trafficking. British Journal of Criminology, 54, 2-19. doi: $10.1093 / \mathrm{bjc} / \mathrm{azt} 060$

Piscitelli, A. (2012). Revisiting notions of sex trafficking and victims. Vibrant: Virtual Brazilian Anthropology, 9, 274-310. doi: 10.1590/S180943412012000100010 
Raymond, J. G. (2002). The new UN trafficking protocol. Women's Studies International Forum, 25, 491-502. doi: 10.1016/S0277-5395(02)00320-5

Regehr, C. \& Jansen, K. (1999). Perceptions of control and long-term recovery from rape. The American Journal of Orthopsychiatry, 69(1), 1105 .

Reid, J. A. (2012). Exploratory review of route-specific, gendered, and agegraded dynamics of exploitation: Applying life course theory to victimization in sex trafficking in North America. Aggression and Violent Behavior, 17, 257-271. doi: 10.1016/j.avb.2012.02.005

Resick, P. (1993). The psychological impact of rape. Journal of Interpersonal Violence, 8(2), 223-55.

Richards, K., \& Lyneham, S. (2014). Help-seeking strategies of victim/survivors of human trafficking involving partner migration. Trends and Issues in Crime and Criminal Justice, 468, 1-10.

Roe-Sepowitz, D., Gallagher, J., \& Hickle, K. (2014). Exploring sex trafficking and prostitution demand during the Super Bowl 2014. Arizona State University School of Social Work: Office of Sex Trafficking Intervention Research.

Roth, S., Wayland, K., \& Woolsey, M. (1990). Victimization history and victim-assailant relationship as factors in recovery from sexual assault. Journal of Traumatic Stress, 3(1), 169-180. doi:10.1002/jts.2490030112

Rothbaum, B., Foa, E., Riggs, D., Murdock, T., \& Walsh, W. (1992). A prospective examination of post-traumatic stress disorder in rape victims. Journal of Traumatic Stress, 5(3), 455-475. doi:10.1007/BF00977239

Ryan, R. M., Deci, E. L. and Vansteenkiste, M. (2016). Autonomy and Autonomy Disturbances in Self-Development and Psychopathology: Research on Motivation, Attachment, and Clinical Process. Developmental Psychopathology. 1(9),1-54.

Saldaña, J. (2009). The coding manual for qualitative researchers. London: Sage. 
Sanghera, J. (2005). Unpacking the Trafficking Discourse. Trafficking and Prostitution Revisited. 9-10.

Saunders, P. (2005). Traffic violations determining the meaning of violence in sexual trafficking versus sex work. Journal of Interpersonal Violence, 20, 343-360. doi: 10.1177/0886260504272509

Scarpa, S. (2008). Trafficking in human beings: Modern slavery. Oxford: Oxford University Press.

Schaeffer-Grabiel, F. (2010). Sex trafficking as the 'new slave trade'? Sexualities, 13, 153-160. doi: 10.1177/1363460709359234

Segrave, M., Milivojevic, S., \& Pickering, S. (2009). Sex trafficking: International context and response. New York: Willan Publishing.

Shamir, H. (2012). A labor paradigm for human trafficking. UCLA Law Review, 60, 76-136.

Sharma, J. (2015) 'Sex Trafficking in Nepal: Women’s Experiences of (re) integration' Doctoral Thesis. University of Queensland.

Shelley, L. (2010). Human trafficking. A global perspective. Cambridge: Cambridge University Press.

Siegel, D. (2012). Mobility of sex workers in European cities. European Journal on Criminal Policy and Research, 18, 255-268. doi: 10.1007/s10610-011-9168-5

Silverman, J. G., Decker, M. R., Gupta, J., Maheshwari, A., Patel, V., Willis, B. M., \& Raj, A. (2007). Experiences of sex trafficking victims in Mumbai, India. International Journal of Gynecology \& Obstetrics, 97, 221226. doi: 10.1016/j.ijgo.2006.12.003

Skeldon, R. (2000). Trafficking: A perspective from Asia. International Migration, 38, 7-30. doi: 10.1111/1468-2435.00113

Skilbrei, M.L., \& Tveit, M. (2008). Defining trafficking through empirical work : Blurred boundaries and their consequences. Gender, Technology and Development, 12(1), 9-30. doi:10.1177/097185240701200103 
Smith, M., \& Kelly, L. (2001). The Journey of Recovery after a Rape Experience. Issues in Mental Health Nursing, 22(4), 337-352.

Soderlund, G. (2005). Running from the rescuers: New US crusades against sex trafficking and the rhetoric of abolition. NWSA Journal, 17, 64-87.

Spohn, C. (2014). The non-prosecution of human trafficking cases: An illustration of the challenges of implementing legal reforms. Crime Law and Social Change, 61(2), 169-178.

Starzynski, L., Ullman, S., \& Vasquez, A. (2017). Sexual assault survivors' experiences with mental health professionals: A qualitative study. Women \& Therapy, 40(1-2), 228-246. doi:10.1080/02703149.2016.1213609

Stoecker, S. (2000). The rise in human trafficking and the role of organized crime. Demokratizatsiya, 8, 129-144.

Stutterheim, S., Sicking, L., Brands, R., Baas, I., Roberts, H., Van Brakel, W., ... Bos, A. (2014). Patient and provider perspectives on HIV and HIVrelated stigma in dutch health care settings. Aids Patient Care and Stds, 28(12), 652-665.

Surtees, R. (2003). Negotiating violence and non-violence in Cambodian marriages. Gender and Development, 11, 30-41.

Surtees, R. (2004). Traditional and emergent sex work in urban Indonesia. Intersections: Gender, History and Culture in the Asian Context (10), Retrieved from http://intersections.anu.edu.au/issue10/surtees.html.

Surtees, R. (2008a). Traffickers and trafficking in Southern and Eastern Europe considering the other side of human trafficking. European Journal of Criminology, 5, 39-68. doi: 10.1177/1477370807084224

Surtees, R. (2008b). Re/integration of trafficked persons: How can our work be more effective. Vienna: Nexus Institute.

Surtees, R. (2008c). Re/integration of trafficking persons: Handling 'difficult' cases. Vienna: Nexus Institute. 
Surtees, R. (2008c). Why shelters? Considering residential approaches to assistance. Vienna: Nexus Institute.

Surtees, R. (2009). Re/integration of trafficked persons: Developing monitoring and evaluation mechanisms. Vienna: Nexus Institute

Surtees, R. (2010). Monitoring anti-trafficking re/integration programmes: A manual. Vienna: Nexus Institute.

Surtees, R. (2012). Re/integration of trafficked persons: Supporting economic empowerment. Vienna: Nexus Institute.

Thompson, M. (2000). Life after rape: A chance to speak? Sexual and Relationship Therapy, 15(4), 325-343. doi:10.1080/713697439

Todres, J. (2005). Importance of realizing other rights to prevent sex trafficking, The Cardozo Journal of Law \& Gender, 12, 885-907.

Todres, J. (2009). Law, otherness, and human trafficking. Santa Clara Law Review, 49, 605-672.

Tummala-Narra, P. (2007). Conceptualizing trauma and resilience across diverse contexts. Journal of Aggression, Maltreatment \& Trauma, 14(1-2), 33-53. doi:10.1300/J146v14n01_03

Tyldum, G., \& Brunovskis, A. (2005). Describing the unobserved: Methodological challenges in empirical studies on human trafficking. International Migration, 43(1-2), 17-34. doi:10.1111/j.00207985.2005.00310.x

Ullman, S. (1997). Attributions, world assumptions, and recovery from sexual assault. Journal of Child Sexual Abuse, 6(1), 1-19.

Ungar, M. (2013). Resilience, trauma, context, and culture. Trauma, Violence, \& Abuse, 14(3), 255-266. doi:10.1177/1524838013487805

UNODC (2003a). Protocol against the smuggling of migrants by sand, sea, and air to the UN convention against transnational organized crime. Retrieved from: http://www.unodc.org/unodc/treaties/CTOC/ 
UNODC (2003b). Protocol to prevent, suppress, and punish trafficking in persons to the UN convention against transnational organized crime.

Retrieved from: http:/www.unodc.org/unodc/treaties/CTOC/

UNODC (2012). Global report on trafficking in persons. New York: United Nations.

UNODC (2013). Issue paper: Abuse of a position of vulnerability and other "means"within the definition of trafficking in persons. New York: United Nations.

UNODC (2016). Global report on trafficking in persons. New York: United Nations.

US Department of State (2013). Trafficking in persons report. Washington DC: US Department of State.

Van Hook, M. P., Gjermeni, E., \& Haxhiymeri, E. (2006). Sexual trafficking of women tragic proportions and attempted solutions in Albania. International Social Work, 49, 29-40. doi: 10.1177/0020872806057086

Väyrynen, R. (2003). Illegal immigration, human trafficking, and organized crime. Helsinki: World Institute for Development Economics .

Verhoeven, M., van Gestel, B., de Jong, D., \& Kleemans, E. (2013). Relationships between suspects and victims of sex trafficking. Exploitation of prostitutes and domestic violence parallels in Dutch trafficking cases. European Journal on Criminal Policy and Research, 1-16. doi: 10.1007/s10610-013-9226-2

Vijeyarasa, R. (2010). The state, the family and language of 'social evils': Restigmatising victims of trafficking in Vietnam. Culture, Health \& Sexuality, 12, S89-S102. doi: 10.1080/13691050903359257

Vijeyarasa, R. (2012, February). The Cinderella syndrome: Economic expectations, false hopes and the exploitation of trafficked Ukrainian women. Women's Studies International Forum, 35, 53-62, doi: 10.1016/j.wsif.2011.09.004 
Vijeyarasa, R. (2013). Stigma, stereotypes and Brazilian soap operas: Roadblocks to ending human trafficking in Vietnam, Ghana and Ukraine.

Gender, Place \& Culture, 20, 1015-1032.

Vindhya, U., \& Dev, V. S. (2011). Survivors of sex trafficking in Andhra Pradesh evidence and testimony. Indian Journal of Gender Studies, 18, 129165. doi: 10.1080/0966369X.2012.759905

Wagenaar, H., \& Altink, S. (2012). Prostitution as morality politics or why it is exceedingly difficult to design and sustain effective prostitution policy. Sexuality Research and Social Policy, 9, 279-292.

doi: 10.1007/s13178-012-0095-0

Weitzer, R. (2007). The social construction of sex trafficking: Ideology and institutionalization of a moral crusade. Politics \& Society, 35, 447-475. doi: $10.1177 / 0032329207304319$

Weitzer, R. (2015). Researching prostitution and sex trafficking comparatively. Sexuality Research and Social Policy, 12(2), 81-91. doi:10.1007/s13178-014-0168-3

Wirth, K. E., Tchetgen, E. J. T., Silverman, J. G., \& Murray, M. B. (2013). How does sex trafficking increase the risk of HIV infection? An observational study from Southern India. American Journal of Epidemiology, 177, 232-241. doi: 10.1093/aje/kws338

Wong, W. C., Holroyd, E., Chan, E. Y., Griffiths, S., \& Bingham, A. (2008). "One country, two systems": Sociopolitical implications for female migrant sex workers in Hong Kong. BMC International Health and Human Rights, 8, 13. doi: 10.1186/1472-698X-8-13

Worthen, M. (2011). Sex trafficking or sex work? conceptions of trafficking among anti-Trafficking organizations in nepal. Refugee Survey Quarterly : Country Reports, Documentation, Literature Survey, Reviews, Vol. 30, No. 3, P. 87-106.

Wright, M., Fopma-Loy, J., \& Fischer, S. (2005). Multidimensional assessment of resilience in mothers who are child sexual abuse survivors. Child Abuse \& Neglect: The International Journal, 29(10), 1173-1193. 
Zhang, S. (2011). Sex trafficking in a border community: A field study of sex trafficking in Tijuana, Mexico. San Diego: San Diego State University.

Zimmerman, C., Hossain, M., Yun, K., Gajdadziev, V., Guzun, N., Tchomarova, M., ... \& Watts, C. (2008). The health of trafficked women: A survey of women entering posttrafficking services in Europe. American Journal of Public Health, 98, 55-59. doi: 10.2105/AJPH.2006.108357

Zimmerman, C., Kiss, L., Hossain, M., \& Watts, C. (2009). Trafficking in persons: A health concern? Ciência \& Saúde Coletiva, 14, 1029-1035. doi: $10.1590 /$ S1413-81232009000400010 


\section{Annexes}

\section{Annex 1}

\section{Some clarifications on terminology}

\section{$(\mathrm{Re})$ integration rather than reintegration}

The term (re) integration will be used throughout this thesis, and (re) deliberately placed in parenthesis. Some of the persons captured in this research were never truly integrated into the society in which they lived, prior to the experience of trafficking. They may have lacked basic personal documents such as birth certificates, ID cards and medical cards. Thus, the efforts following the trafficking experience were of integration, rather than reintegration.

\section{Victim, survivor, beneficiary are used interchangeably.}

The researcher is fully aware of the discussion amongst academics and practitioners, to avoid the use of the term 'victim' and use the term 'survivor' in its place. However, for the purpose of this study, these terms will be used interchangeably, as well as most often, the term 'trafficked person' to refer to anybody belonging to the main target group of the study. The reason for this is the fact that not all women may prefer to be called 'survivor', and even further, this term may not be a good descriptor of their current situation. It may be that for some women, using the term 'victim' is a recognition of the suffering they have endured. Finally, in certain cases, and countries, a person must be identified as a 'victim' in order to be recognised as a person trafficked. Although it should be said that the term most commonly used by organisations and service providers working with trafficked persons is 'beneficiaries'. Nonetheless, this study will use the term 'victim', 'survivor', 'trafficked person' and 'beneficiary', in order to refer to those belonging to the main target group. The interchangeability of these terms in this context should be taken to mean that this group of individuals should not be solely labeled as one or the other, and what term is used should ultimately be up to the person to whom it refers, depending on their context and circumstances. (Chapter 3: Methodology) 


\section{A deliberate decision was made to use the term 'sex work' and not 'prostitution'}

At the beginning of this research, both terms 'sex work' and 'prostitution' were used together. The intention was to be objective, and give equal space to the 'sex workers' rights camp and the abolitionist camp. However, after the data was analysed and Chapter 7 was written, which shows the potential harm of stigmatisation, when using terms such as 'prostitution', this researcher, at the advice and direction of her supervisors, has adopted the use of 'sex work' exclusively. The intention is still not to take any particular side, in the pro and anti sex work debates. However, what is clear is that stigma, partially perpetuated through the use of terms such as 'prostitution' does harm to the very people for whom this research was conducted, and intended to help. Thus, the only chapter in which the word 'prostitution' remains is Chapter 2, which was written and published as is, at the very beginning of this research.

\section{Long-term (re) integration}

When the term 'long-term (re) integration' is used throughout this research, it is meant (re) integration after the full 3 year monitoring and evaluation phase is completed, and the time period after which trafficked persons no longer have any official contact with assistance programs. 


\section{Annex 2}

\section{Interview Guide - Trafficked Persons}

\section{Guideline:}

Overall themes to be covered: bullet point, black

Main questions to be asked: bold, capital, within borders

Sub-questions: red

Clarification of sub-questions: red, italics

\section{- General Information}

-age

-current place of residence

-level of education / profession/previous training

-children? Married/living together?

-time passed since end of trafficking/end of time at Shelter/end of services provided by assistance programmes?

\section{- PRE-TRAFFICKING INFORMATION}

\section{MAIN QUESTION: CAN YOU DESCRIBE YOUR LIFE BEFORE} THE TRAUMATIC EVENT (IF POSSIBLE, SAY TRAFFICKING)?

-accommodation status

Where did you live?

-employment status

Were you working? Or studying?

-contacts with family and friends

Can you describe your interactions/relationship with family?

Can you describe your interactions/relationship with friends?

What did you do during the week, for entertainment?

What did you do during the weekend for entertainment?

Can you describe any interactions with community members?

Was religion a part of your life?

-physical, mental health/any prior abuse

Have you suffered any physical/sexual or mental abuse in the past? 
Did you feel physically healthy at this time?

Did you feel mentally healthy at this time?

(all questions pertaining to mental/physical health prior to trafficking may be posed to social worker/case manager)

- TRAFFICKING EXPERIENCE

MAIN QUESTION: WHAT CAN YOU TELL ME ABOUT THE TRAUMATIC EVENT (TRAFFICKING EXPERIENCE)? HOW DID IT HAPPEN? WHAT GOT YOU THROUGH IT? (IF NECESSARY, INFORMATION FOR THIS SEGMENT WOULD BE GATHERED FROM SOCIAL WORKER/CASE MANAGER)

-motivation that led to actions which led to trafficking

-recruitment - were family and /or friends involved?

-age when trafficked

-length of trafficking experience

-severity of trafficking experience

-manner of exiting trafficking experience

-danger of being re-trafficked

How old were you when this experience happened?

Can you describe how it occurred?

(Were there certain factors that were decisive in pushing you along this path?)

Who was the person/s involved?

Was anybody that you knew personally involved?

(Is there anybody/anything that you hold most responsible for what happened?)

If you think back to the traumatic experience today, how would you describe it?

(Was there anybody/anything giving you hope and energy to survive through it?) 
Did you feel like a victim?

How did it come to an end?

How did you survive throughout the traumatic period?

Did receive any financial reimbursements during?

(Did you send any money back to your family?)

Did you have any contact with your family or friends during?

- IDENTIFICATION

\section{MAIN QUESTION: HOW WERE YOU IDENTIFIED AS} TRAFFICKED?

Did you realize from the very beginning that you were a person trafficked? What do you think about the term 'trafficked'? Do you feel it applies to your case?

- Post-TRAfFicking

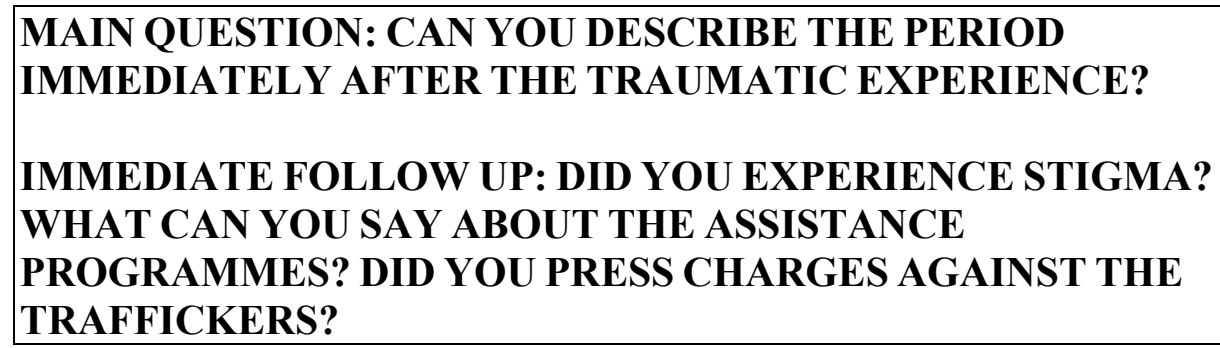

Can you describe what happened in the period immediately after the trafficking experience?

In general, looking at the period immediately following the trafficking experience, what would you say was of greatest help for you? What was the greatest hindrance?

What motivated you?

Can you think of a situation in which you experienced stigma,

discrimination or a negative reaction due to your trafficking experience?

Please describe the situation.

How did this experience affect you?

How did you react to this experience?

Did you feel like you had control over what was happening to you immediately after the trafficking experience?

Did you feel safe? 
What do you feel when you think back to this period?

(Did you have financial security at this moment? Social support? Did you deal with any mental or physical consequences?)

\section{Participation in (re)integration programmes}

-What types of programmes are available for trafficked women?

-What are the conditions for participation in such programmes?

-Do women practice agency when they participate in assistance programmes?

-What is the level of participation of women in the design of their individual (re)integration plans?

Did you participate in any (re)integration programme? (or any other type of social programme?)

(What motivated you to participate?/What motivated you to decline?)

What kind of programmes did you participate in?

How did you get involved in this program?

Were there any conditions for participating in such a programme?

What was your role in this process?

Did you participate in the design of the assistance offered to you?

Can you describe your relationship with some of the service providers?

Can you describe your relationship with the other women in the programme? (Do you still keep in touch?)

Overall, were you satisfied with the experience? What was the greatest benefit, if any? What did you not like from the overall experience?

- CURRENT ECONOMiC SITUATION

MAIN QUESTION: CAN YOU DESCRIBE YOUR CURRENT ECONOMIC SITUATION? 


\section{Economic situation}

- Employment status: perceived need versus current employment status

-Are you currently employed? (if not, are you happy with the current situation?)

-Are you happy with your current job?

-What would be your ideal job?

- Accommodation status: perceived need versus current housing situation

-Where do you live at the moment?

-Do you live alone or with family/friends/roommates?

-Are you happy with your current living situation?

-What would be your ideal living situation?

Financial status: perceived need versus current financial situation

-What is your current financial situation?

-Are you satisfied with your current financial situation?

What would you say is being economically stable?

- CURRENT SOCIAL AND HEALTH SITUATION

MAIN QUESTION: CAN YOU DESCRIBE YOUR CURRENT SOCIAL LIFE? (INTERACTIONS WITH FAMILY, FRIENDS, COMMUNITY?)

MAIN QUESTION: PHYSICALLY, PSYCHOLOGICALLY, HOW DO YOU FEEL TODAY?

\section{Social state of being}

- Family (parents, romantic relationship, children)

-Is there contact with family members, how often?

-Do current relationships satisfy perceived needs? 
Who would you consider as being part of your family today?

Are you in contact with them?

How often?

How would you describe your relationship?

Do you feel supported by your family?

Is this type of relationship satisfying to your needs?

Are family members aware of your trafficking experience? Why yes/why not? (If yes, what was their reaction?)

Does this create challenges for the relationship?

What do you think their understanding of trafficking is?

How do you think your family views persons that have been trafficked?

- Friends

-How many friends does the woman have and how often is she in touch with them?

-Do current relationships satisfy perceived needs?

Who would you consider as a friend today?

Are you in contact with them?

How often?

How would you describe your relationship?

Do you feel supported by your friends?

Is this type of relationship satisfying to your needs?

Are friends aware of your trafficking experience? Why yes/why not? (If yes, what was their reaction?)

Does this create challenges for the relationship?

What do you think their understanding of trafficking is?

How do you think your friends view persons that have been trafficked? 


\section{- Community members}

-Is there interaction with neighbors, and other members of community and how often?

-Do current interactions satisfy perceived needs?

What role does your community play in your life today?

How often do you come in contact with neighbors, members of your community?

Do you participate in any community sport/social activities?

Is this a satisfactory situation for you?

Do you feel a part of your community today?

Is anybody from your community aware of your trafficking experience?

If they are aware, what is their reaction? How do they treat you?

How do you think trafficking is understood in your community?

How do you think are trafficked persons viewed within your community?

\section{- Religious groups}

-Is there interaction with any religious groups and how often?

-Do current interactions satisfy perceived needs?

What role does religion play in your life today?

Did the Church provide support to you, or you felt like they condemn you for what happened?

Is this relationship satisfactory to you?

What do you do during the week, for entertainment?

What do you do during the weekend for entertainment? 


\section{Family/friends/community -in depth inquiry ( $a, b$, COVERED ABOVE)}

(a) Are the family/friends/community aware of the trafficking experience?

(b) If yes/no, does this create significant challenges for the relationship?

(c) Perception of stigmatization: family, friends, community members

(c) Can you think of a situation in which you experienced stigma, discrimination or a negative reaction due to your trafficking experience? Please describe the situation. How did this experience affect you?

How did you react to this experience? (coping)

What would you say is being socially included?

\section{Physical and mental state of being}

- Physical well being

- Anxiety and fear (danger of being re-trafficked)

- Depression

- Post-traumatic stress disorder (PTSD)

- Eating disorders

- Substance abuse

\section{Beliefs/Behavior/attitude}

- Agency

- Coping

-Are there any approach or avoidance coping strategies present? 
Do you feel physically healthy today?

Are there any permanent physical/behavioral consequences of the trafficking experience? (are there any eating disorders? Substance abuse?) What would you say is being in a good health condition - physical and mental?

How often do you think of the trafficking experience today?

What are some of the feelings that you experience when thinking of it? (Anxiety? Fear? Do you feel depressed?)

How do you deal with some of these feelings?

(What helps and what hinders coping with these experiences on a daily basis?)

Did (and if yes, how) your approach/feelings/attitude toward the trafficking experience change with time?

Do you feel in control of your life today?

Are you happy with your life today?

What would you say is of greatest value to you today?

(What gives you hope?)

What scares you?

WHAT MOTIVATES YOU?

- FUTURE; RAPPING UP

\section{MAIN QUESTION: CAN YOU DESCRIBE HOW YOU ENVISION THE FUTURE?}

Do you think about the future?

(Can you describe some of your ideas about the future?)

What are some of your goals for the future?

Overall, and in conclusion, what is in your opinion of greatest importance for achieving well-being (creating a well rounded life)?

If there is time:

- RE(INTEGRATION)

-What does re(integration) mean to you?

-When would you say re(integration) has been successful?

(Is it a matter of time, or certain supporting factors have to be present?) 


\section{- Questionnaire}

- What do you think about this questionnaire?

\section{Interview Protocol - Service Providers}

Name:

Title:

Organization/Institution:

- Can you tell me about your daily work? How does a normal day look like?

- How do you come in contact with trafficked women?

How were they identified?

How are they referred to you?

- What is your approach, when you come in contact with a trafficked woman for the first time?

What are the needs of the women?

What do they usually want from you?

What do they tell you?

What do you tell them?

How do you interact with them?

What are challenges during the first contact with a trafficked woman?

- What is the importance of the criminal trial? Financial compensation?

- How do you proceed as time goes by?

How do the needs and wishes of trafficked women change with time?

How long do you follow up on each case? / How do you interact with them as time goes by? 
What are some of the challenges in your later interactions with trafficked women?

- What in your opinion constitutes successful (re)integration of a trafficked woman?

- What is the importance of the criminal trial? Financial compensation?

- Can you give me some concrete examples from your personal experience?

Examples when (re)integration was successful?

Examples when (re)integration was not successful?

- Can you give one or more examples of women who have gone back to prostitution/sex work, after trafficking?

What can you say about their recovery and reintegration, in this case?

How would you say professionals in your field (other social workers/psychologist working with trafficked persons) respond to women that would like to return to sex work after a trafficking experience?

Does this differ from your own views?

How would you say professionals in your field approach women that state they have entered prostitution voluntarily?

Does this differ from your own views?

What kind of skills do you think professionals that work with trafficked persons should have?

Does your institution/organization have a particular policy toward prostitution/sex work?

Additional question: what is human trafficking? what is sex work?; do you make a distinction? 


\section{Annex 3}

Organisations and institutions contacted:

Albania:

Tjeter Vizion, Elbasan

Vatra, Vlore

Bosnia Herzegovina:

Emmaus, Sarajevo

Medica, Zenica

Bulgaria:

Animus, Sofia

IOM, Sofia

PULSE Foundation, Pernik

\section{Montenegro:}

Office to Fight Human Trafficking General Secretariat

Female NGO Lobby, Podgorica

\section{Serbia:}

NGO Astra, Belgrade

NGO Atina, Belgrade

Centre for Victim Protection, Belgrade

Netherlands:

Prostitutie\&Gezondheidscentrum, Amsterdam

Amsterdams Coordinatiepunt Mensenhandel (ACM), Amsterdam

Italy:

Farsi Prossimo, Milano 


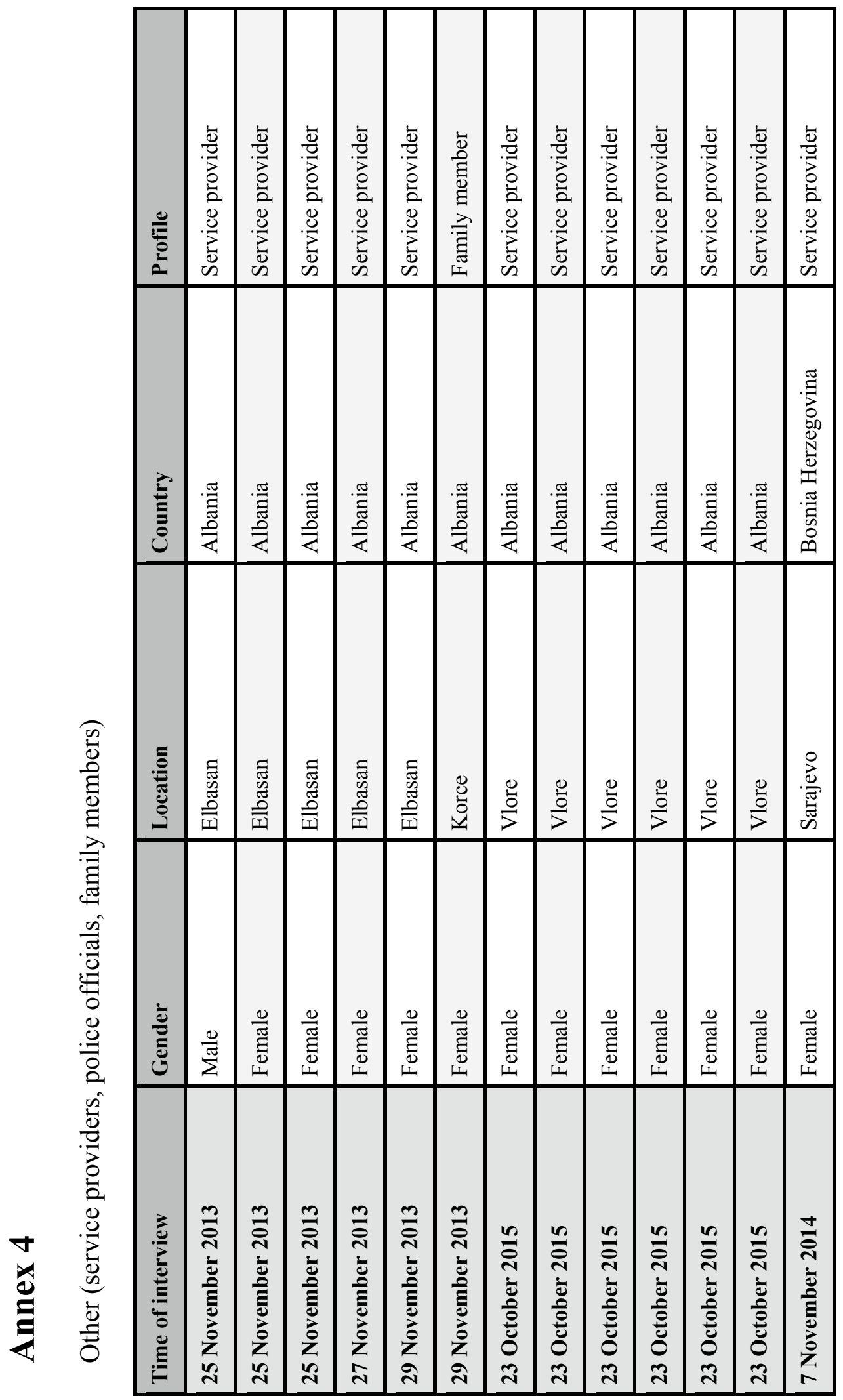




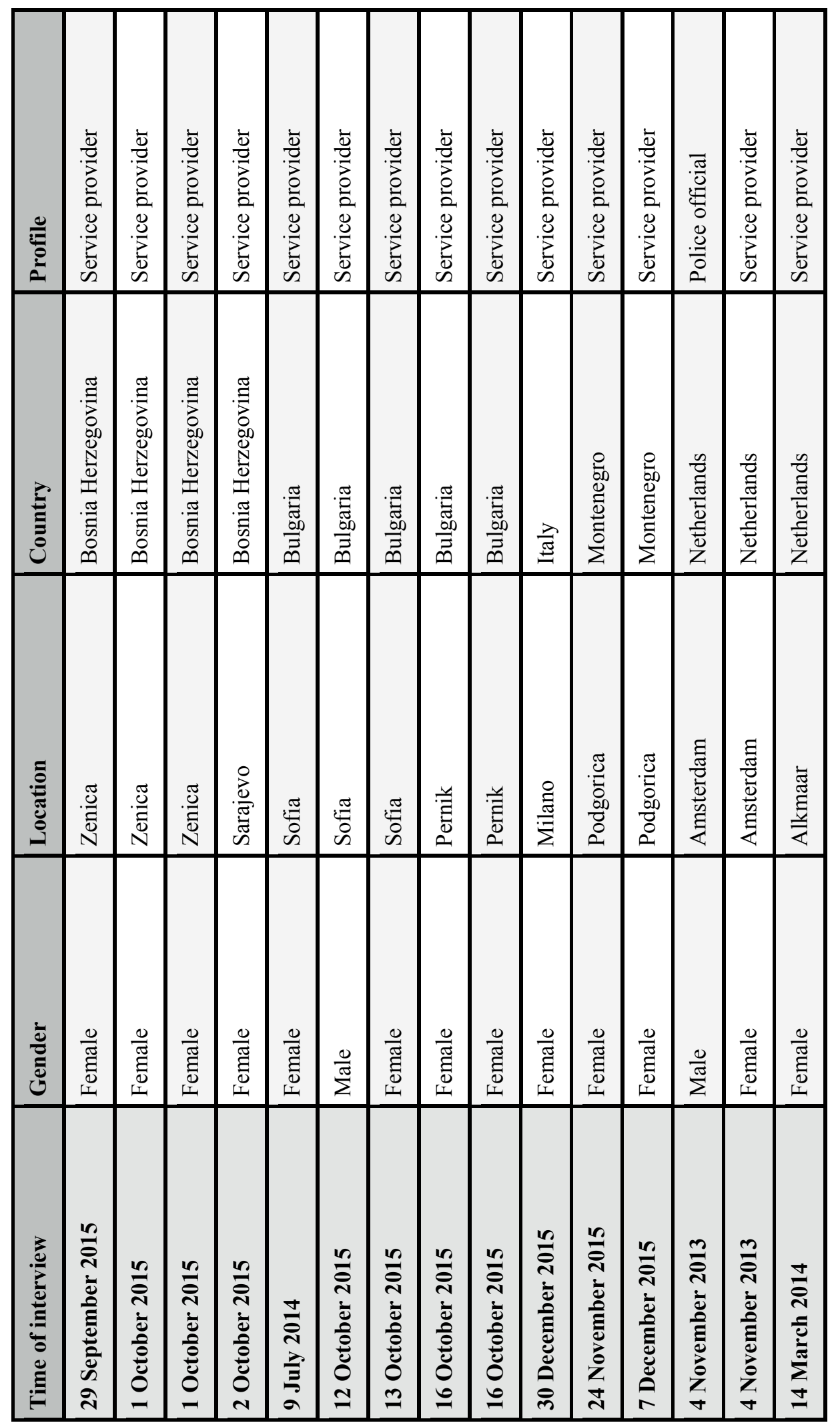




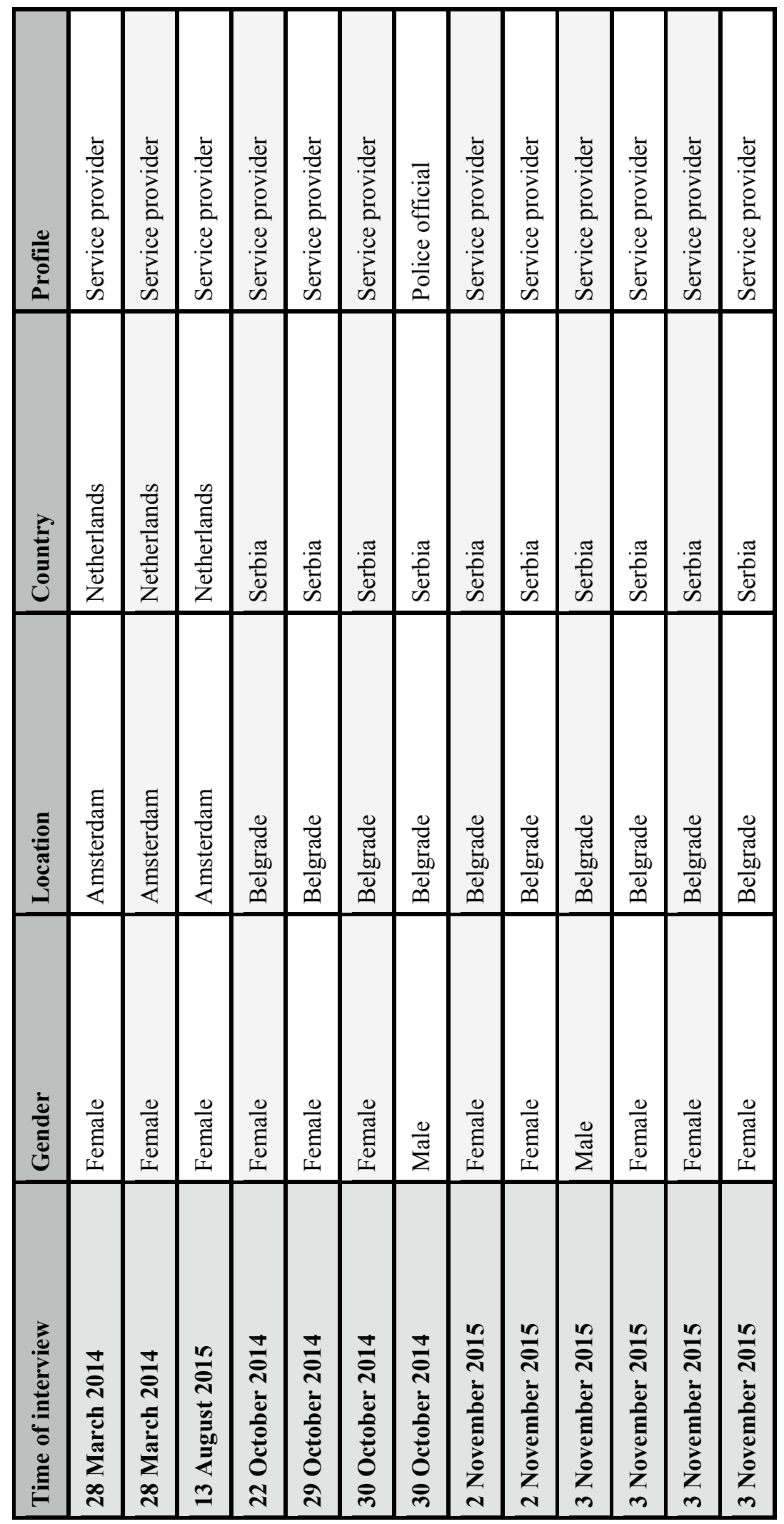




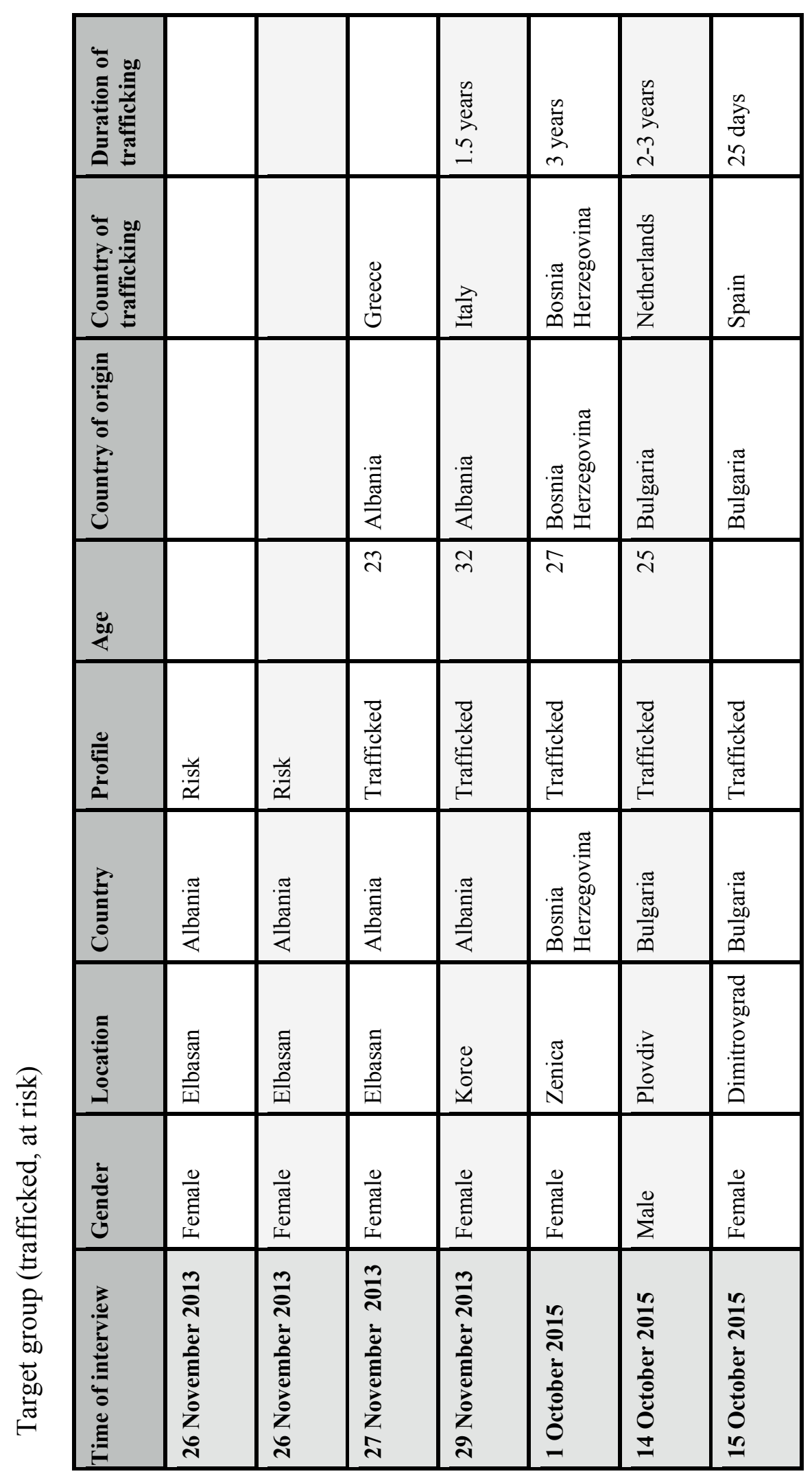




\begin{tabular}{|c|c|c|c|c|}
\hline 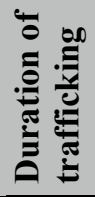 & & $\begin{array}{l}\frac{n}{0} \\
0 \\
0 \\
N \\
N\end{array}$ & 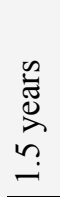 & $\underset{\sim}{\stackrel{D}{d}}$ \\
\hline 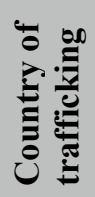 & & 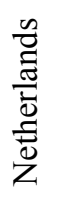 & 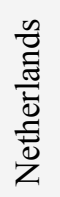 & 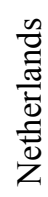 \\
\hline 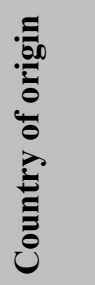 & 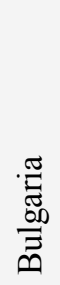 & 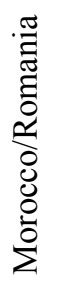 & 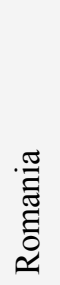 & 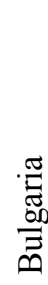 \\
\hline 总 & & N & $\stackrel{\sim}{\forall}$ & $\stackrel{n}{2}$ \\
\hline 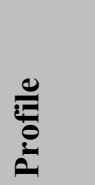 & 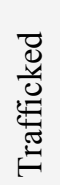 & 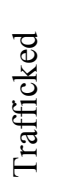 & 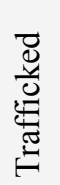 & 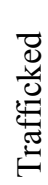 \\
\hline$\underset{\Xi}{己}$ & 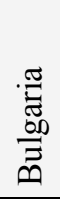 & 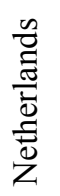 & $\begin{array}{l}\frac{n}{0} \\
\frac{\pi}{0} \\
\overline{0} \\
\overline{0} \\
Z\end{array}$ & $\begin{array}{l}\frac{n}{0} \\
\frac{\pi}{0} \\
\overline{0} \\
\overline{0} \\
\overline{0}\end{array}$ \\
\hline 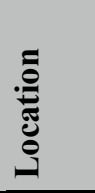 & 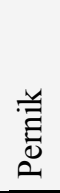 & 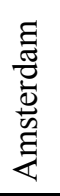 & 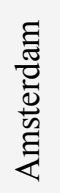 & 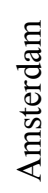 \\
\hline نั & $\begin{array}{l}\frac{0}{\widetilde{J}} \\
\text { đ̃ } \\
\text { I }\end{array}$ & $\begin{array}{l}\frac{0}{\widetilde{J}} \\
\text { U్d } \\
0 \\
\text { I }\end{array}$ & 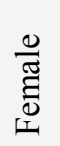 & $\begin{array}{c}\frac{0}{\widetilde{J}} \\
\stackrel{0}{0} \\
\text { İ工 }\end{array}$ \\
\hline 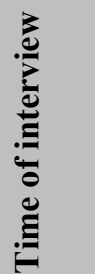 & 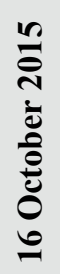 & 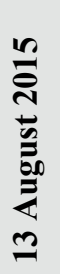 & 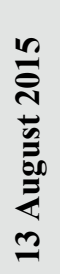 & 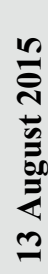 \\
\hline
\end{tabular}




\section{Valorisation}

\section{Background}

This thesis answers the question of what determines successful recovery and (re) integration of women trafficked for the purpose of sexual exploitation, and how influencing factors interplay, in the short and long run. The analysis is based on qualitative research conducted in Southeastern Europe (Albania, Bulgaria, Bosnia Herzegovina, Montenegro and Serbia) and Western Europe (Netherlands and Italy).

Much of research on the topic of (re) integration of trafficking victims has focused on the immediate aftermath of being identified as a victim of trafficking. Many of the (re) integration services which are offered to victims address immediate needs, although some programmes follow victims for several years. However, recovery and (re) integration is a long term process, which for some survivors may entail lifelong efforts. What this thesis addresses, is the (re) integration process in its entirety, and determines what some of the long term needs of victims may be, as well as what outcomes ensure the sustainability of a more successful (re) integration process. In addition, this thesis tries to escape the mostly polemic discussions around the issue of sex work, and instead, through empirical evidence looks at the influence attitudes toward sex work as well as existing stigma toward the issue may have on the overall (re) integration process of trafficking victims.

\section{Relevance and innovativeness}

There are a number of ways in which this thesis has made a contribution to research in the field of human trafficking.

\section{Methodological}

When research on human trafficking attempts to address any issue which concerns the survivors, efforts are usually made to capture the views of the target group. In this process, there are possibly two consequences. Firstly, involving human trafficking victims in research on the topic may in some way re-trigger past experiences, and thus, there is always the danger that victims 
may be re-traumatised. This is precisely why many organisations that offer (re) integration services have introduced strict policies where they do not make their beneficiaries accessible to researchers. Secondly, in the attempts to capture the views of survivors, researchers often miss another highly significant group which may be a source of valuable information, namely services providers. It is precisely this group that has been captured in this research. Service providers, some of whom have been doing their job for many years, have information on the (re) integration process of multiple beneficiaries. Additionally, they also have information on the (re) integration process over time. Capturing the needs of survivors many years after having been identified as trafficked is particularly difficult. Victims usually do not want to speak or remember the traumatic experience of the past, and some of them have cut all communication with any actor from that part of their life, including the organisations which offered (re) integration services. Individual survivors who do remain in touch with service providers, are often reluctant to speak to researchers. Thus, through speaking to service providers, this thesis has identified some of the needs of victims several years after they have exited official (re) integration programmes.

\section{Geographical}

Research on (re) integration of persons trafficked for the purpose of sexual exploitation usually focuses on the return of women in their country of birth. Women either express the wish that they would like to go back home, or have no choice as they do not have legal right and documentation to stay in the country to which they were trafficked. However, there is always a group that does remain in the country in which they were identified. Little is known of the recovery and (re) integration processes of this group. In addition to looking at (re) integration processes of women that did return to their country of birth, this research has also looked at the recovery and (re) integration of women that have remained in the country in which they have been identified - in this case the Netherlands and Italy. The differences between origin and destination countries were found to be particularly stark in regard to the way criminal proceedings against the trafficker are conducted, and the treatment of the victim throughout this process (Chapter 6). Thus, whereas criminal proceedings against traffickers in origin countries are long, over many years, often re-traumatise, and almost never offer financial compensation, in the Netherlands, trials are significantly faster, at times when the victim is too vulnerable do not request her testimony and almost always entail financial compensation for the survivor. On the issue of stigmatisation of sex work and 
by extension of victims of trafficking for the purpose of sexual exploitation, this research has found similarities, rather than differences between origin and destination countries, meaning these two groups are stigmatised in both the country of their birth, as well as in the country in which they were identified (Chapter 7).

\section{Conceptual}

The main concept which has been addressed throughout this thesis is that of (re) integration, and how the process of recovery fits within that context, for victims of trafficking for the purpose of sexual exploitation (in particular in Chapter 4). Thus, this thesis has found that we cannot speak of definitive success of the (re) integration process, but a continuum of success along different dimensions, which were also identified and discussed (within the recovery process, economic, social and institutional dimensions). Even further, it has determined what gives sustainability to the process of (re) integration in the long run, which is the building and re-building of healthy relationships, and formation of a new family. This is not seen as a loss of independence, but as having the autonomy to make decisions which ultimately satisfy the need to belong, and contribute to the long process of recovery and (re) integration. Thus, a key distinction is made between the concepts of autonomy and independence. Namely, autonomy is what is seen as desirable, the ability to make one's own decisions. As long as the survivor is free to practice her autonomy in every area of her life, is no longer in a situation of exploitation, independence is seen as largely not relevant.

\section{Empirical}

(Chapters 4, 5) One of the main findings of this research is that the ability of forming healthy relationships, personal in which the survivor can practice her autonomy, and relationships with service providers, built on trust, are the most significant factors that could contribute toward successful long term (re) integration of trafficking victims. As noted, significance of the newly formed family, following a trafficking experience is identified as an important factor influencing the success and sustainability of the (re) integration process. Of course, for this to be achieved, and for the survivor to have the ability to reestablish healthy relationships, other factors are also important, especially in the short term, upon being identified as a victim of trafficking. Thus, therapy is crucial, as well as being given clear, open and honest information from service providers on immediate steps that would follow after being 
identified as a victim of trafficking. Such exchanges would work toward reestablishing trust that the victim has lost in social relations.

(Chapter 6) When looking at the dynamics of the prosecution of the trafficker, it was found that trials often last a long time, and may require the victim testifying numerous times, which often causes re-traumatisation. Thus, although conviction of the trafficker may satisfy needs for justice and safety of some victims of trafficking, it is in situations where the prosecution and trial are swift that this is most pronounced. This thesis has also found that there are significant variations amongst countries as to the possibility of financial compensation of victims. Thus, countries such as the Netherlands have made the option of pursuing financial compensation for survivors easily attainable. In addition, most persons recognised as victims of trafficking, whose trafficker is successfully prosecuted, are granted financial compensation. This is not the case in most origin countries studied in the context of this research.

(Chapter 7) Finally, when looking at the approach to sex work in the context of anti-trafficking, this thesis finds ongoing stigmatisation, not only of sex workers, by both service providers and victims of trafficking, but also reports of stigmatisation of trafficking victims. Stigmatisation is due to the association of trafficking victims, with the issue of sex work, and also because of the lack of understanding of the differences between sex work and sex trafficking. Such stigmatisation has negative consequences for the (re) integration process of survivors.

\section{Target audience}

Much of the funds allocated to (re) integration services for trafficking victims and much of the type of (re) integration services available at the moment focus on the immediate aftermath of being identified as a trafficking victim. In addition, a lot of attention is being placed on the perceived need of particularly economic independence of trafficking victims. What this thesis has shown however, is that what seems to offer longevity to the potential success of the recovery and (re) integration process is the ability of survivors to form healthy relationships based on trust. In particular formation of new families, as well as sustaining a relationship with a service provider who has earned the trust of the survivor. Thus, much more attention should be given to these areas of the (re) integration process. In addition, it has been determined that victims are often in the danger of being traumatised again 
throughout the criminal proceedings against the trafficker, with little possibility of subsequent financial compensation for the survivor. Finally, it has been determined that stigmatising attitudes toward sex workers persist in current anti-trafficking organisations, attitudes which are often reflected also by trafficking victims. These may extend to the group of trafficked persons themselves, and thus negatively influence the recovery and (re) integration process. With this in mind, there are several groups in addition to academia, that would be the target audience of this thesis.

Primarily, the target of this thesis are all anti-trafficking organisations (national and international, governmental and non-governmental) who seek to offer recovery and (re) integration services to trafficking victims. The strong recommendation to these groups, in particular in Europe, would be to place significantly more attention to programmes that would assist victims in re-building their feelings of trust toward others. As well as programmes that would focus on the establishment of new, healthy relationships. At the moment, the decision about openness of victims about their past experience to newly formed relationships is largely left up to the victim. However, some victims may want more assistance and guidance in regard to this issue, in particular if this is something that is addressed and worked through in therapy sessions, and survivors are assured that they are not to blame for what has happened to them. In addition, anti-trafficking organisations need to give significantly more attention to training of their own staff, and in general sensitivity training of all individuals who may in any way come in contact with trafficking survivors. It has been found that stigmatising attitudes toward sex work extend toward the issue of sex trafficking as well, and thus influence the recovery and (re) integration process in a negative way. Thus, this is an issue that needs to be addressed within these organisations.

Finally, this thesis seeks to reach the judicial branch in origin countries in particular. The current length of criminal proceedings against traffickers are simply unacceptable. In addition, stigmatisation and maltreatment of victims exists in in the context of these trials as well, which in addition to the retelling and re-living of their experiences, further contributes to their revictimisation. Sensitivity training of all members of the judicial branch who may come in contact with trafficking victims, is greatly needed in this area. 


\section{Outreach}

With the completion of the writing of this thesis, several outreach activities have already been undertaken, and will be undertaken in the upcoming period, to ensure that the research does reach the target audiences identified. This thesis has produced a total of six articles, two of which (Chapter 2, Chapter 6) have already been published. The remaining are also being prepared for submission to peer-review journals, and will subsequently be published. Of particular academic value is Chapter 3 which discusses the many methodological challenges that arise when studying a topic such a human trafficking, specifically trafficking for the purpose of sexual exploitation.

In addition, this thesis will be disseminated to all service providers who have participated in this research. As already noted these are individuals who work in anti-trafficking organisations, government bodies, shelters as well as police officials. Each of these actors has significant opportunity and possibility to implement the recommendations proposed by this research, and to take into consideration the results, in order to inform their future work.

Finally, a list of additional relevant actors in the field of anti-trafficking has been drafted, from all the countries that were involved in this research, but who did not participate in it. The thesis will also be disseminated to the identified individuals and organisations that may further be able to take into consideration the results of this research and implement the recommendations in the area of anti-trafficking. 


\section{About the author}

Biljana Meshkovska received her BA from the American College in Thessaloniki (ACT), Greece with a major in History and International Relations and a double concentration in Foreign Affairs and International Relations and in Balkan and European Studies. She received her MA from the School of Advanced International Studies (SAIS), Johns Hopkins University in International Relations and International Economics with a double concentration in International Development and International Economics. While at SAIS she was introduced to the topic of human trafficking, which ultimately became the focus of her current research. She became a PhD research fellow at UNU-Merit/Maastricht Graduate School of Governance in 2012. The particular topic of her studies has been trafficking of women for the purpose of sexual exploitation, with a geographical focus on Europe. 



\section{UNU-MERIT/MGSoG Dissertation Series}

2018

\section{Vincenzo Vinci}

The Relevance of Institutions and

People's Preferences for Social

Protection

UNU-MERIT/MGSoG Dissertation Series № 213

\section{Silke Heuser}

The Effectiveness of Environmental

Policies on Reducing Deforestation in

the Brazilian Amazon

UNU-MERIT/MGSoG Dissertation

Series № 212

\section{Jennifer Waidler}

Social Assistance and Remittances and Their Role in the Fight Against

Poverty

UNU-MERIT/MGSoG Dissertation Series № 211

\section{Choolwe Muzyamba}

The role of community mobilization in the promotion of maternal health of women living with HIV in Zambia UNU-MERIT/MGSoG Dissertation Series № 210

\section{Juan Carlos A. Castillo Sánchez}

Assessing the Role of the Export Sector in Mexican Economic

Development,1965-2014

UNU-MERIT/MGSoG Dissertation Series № 209

\section{Tareq Abuelhaj}

Food Security Policy Impact Analysis:

The Econometrics of Cash and Food Assistance Cost Effectiveness

UNU-MERIT/MGSoG Dissertation

Series № 208

Marta Férnandez de Arroyabe Arranz

Essays on MEAS and Innovation UNU-MERIT/MGSoG Dissertation Series № 207

\section{Clotilde Mahé}

Essays on Migration and Occupational Choice

UNU-MERIT/MGSoG Dissertation

Series № 206

\section{Simone Sasso}

Talent on the move. Essays on Human Capital, Graduate Mobility and

Economic Development

UNU-MERIT/MGSoG Dissertation

Series № 205

\section{Khaled Walid Rajab}

Strategic Planning under Fragility

UNU-MERIT/MGSoG Dissertation

Series № 204

\section{Mutinta Hambayi Nseluke}

A Tall Order: Improving Child Linear Growth

UNU-MERIT/MGSoG Dissertation Series № 203 
Elvis Korku Avenyo

Innovations and Firm Performance

in sub-Saharan Africa: Empirical

Analyses

UNU-MERIT/MGSoG Dissertation

Series № 202

\section{Ni Zhen}

Employment Dynamics, Firm

Performance and Innovation

Persistence in the Context of

Differentiated Innovation Types:

Evidence from Luxembourg

UNU-MERIT/MGSoG Dissertation

Series № 201

\section{Caroline Wehner}

Too Scared to Achieve: The Relation

Between Neuroticism,

Conscientiousness

and Socioeconomic Outcomes

UNU-MERIT/MGSoG Dissertation

Series № 200

\section{Stefania Innocenti}

On Institutional Persistence

UNU-MERIT/MGSoG Dissertation

Series № 199

\section{Hassen Abda Wako}

Economic Globalization, Institutions

and Development: Essays on Aid, Foreign Direct Investment and Trade UNU-MERIT/MGSoG Dissertation Series № 198
2017

\section{Hans-Erik Edsand}

Winds of Change

UNU-MERIT/MGSoG Dissertation

Series № 197

\author{
Ana Patricia Silva Vara \\ Redressing the Gender Gap \\ UNU-MERIT/MGSoG Dissertation \\ Series № 196
}

\section{Andrés Iván Mideros Mora}

Essays on the Economic Effects of Noncontributory Social Protection

UNU-MERIT/MGSoG Dissertation

Series № 195

\section{Tobias Broich}

New Actors in the Global Economy

UNU-MERIT/MGSoG Dissertation

Series № 194

\section{Bernard Nikaj}

From No-government to E-government UNU-MERIT/MGSoG Dissertation

Series № 193

\section{Ali Safarnejad}

Prioritizing the HIV Response

UNU-MERIT/MGSoG Dissertation

Series № 192

\section{Clovis Freire}

Diversification and Structural

Economic Dynamics

UNU-MERIT/MGSoG Dissertation

Series № 191 


\section{Michael Verba}

Innovation and Knowledge Dynamics:

Essays on the Knowledge Economy

UNU-MERIT/MGSoG Dissertation

Series № 190

\section{Pui Hang Wong}

The Hearts and Minds in Conflict and

Peace: The Economics of

Counterinsurgency and the Psychology of Reconstruction

UNU-MERIT/MGSoG Dissertation

Series № 189

\section{Brenda Yamba}

Schooling Despite All Odds: Evidence

from Lesotho on Female Child Carers who Stayed in School

UNU-MERIT/MGSoG Dissertation

Series № 188

\section{Sheng Zhong}

Moving towards An Energy Efficient

Future: Essays on Energy Efficiency,

Technology and Development

UNU-MERIT/MGSoG Dissertation

Series № 187

\section{Julieta Marotta}

Access to Justice and Legal

Empowerment of Victims of Domestic

Violence through Legal Organizations

in the City of Buenos Aires: A

Qualitative Empirical Legal Study

UNU-MERIT/MGSoG Dissertation

Series, № 186

\section{Andrea Franco-Correa}

On the Measurement of

Multidimensional Poverty as a Policy

Tool: Empirical Applications to Chile,

Colombia, Ecuador and Peru

UNU-MERIT/MGSoG Dissertation

Series, № 185

2016

\section{Yesuf Awel}

Insurance for Growth: Empirical

Essays on Insurance Demand and

Impacts in Africa

UNU-MERIT Dissertation Series,

№ 108

\section{Tigist Mekonnen Melesse}

Grow More Food using Fewer

Resources: Agricultural Technology

Adoption and Innovation Practices for

Inclusive and Sustainable

Development

UNU-MERIT Dissertation Series,

№ 107

\section{Eleni Yitbarek}

Getting Ahead or left Behind? Essays on Poverty Dynamics and Social

Mobility in Africa

UNU-MERIT Dissertation Series, № 106

\section{Thuy Dieu Nguyen}

Firm-Level Theory and Evidence of

Corruption

UNU-MERIT Dissertation Series, № 105 


\section{Raquel Tsukada Lehman}

Essays on Household Production with

Labor-Saving Technology

UNU-MERIT Dissertation Series, № 104

\section{Eva Barteková}

Multi-Problem Challenges for a

Renewable Future: Empirical Studies on Competitive Disadvantages from

Electricity Price Differentials and

Mineral Supply Risk in an Open

Economy

UNU-MERIT Dissertation Series, № 103

\section{Jocelyn Olivari}

Entrepreneurial Traits and Innovation:

Evidence from Chile

UNU-MERIT Dissertation Series,

№ 102

\section{Muhammad Shafique}

Essays on the role of knowledge, RED, and Technology-based Firms in the Evolution of Socio-techno-economic System

UNU-MERIT Dissertation Series, № 101

\section{Serdar Türkeli}

Governance of Innovation Policy:

Empirical Studies on Applied Political Economy by Multi-Methods Analysis UNU-MERIT Dissertation Series, № 100

\section{Ayokunu Adedokun}

Pathways to Sustainable Peace

building in Divided Societies: Lessons

and Experiences from Mozambique

MGSoG Dissertation Series, № 75

\section{Luiz Rothier Bautzer}

Organizing Concurrent Engineering through ICT Platforms

Blueprinting Product Lifecycle

Management Platforms across

Disciplinary Agencies

MGSoG Dissertation Series, № 74

\section{Natalia Popova}

Migration in the Periphery of the

European Union:

Determinants of Successful and

Sustainable Labour Market Integration of Return Migrants in Albania, Egypt, Moldova and Tunisia

MGSoG Dissertations Series, № 73

\section{Richard A. Martina}

Uncertainty and Resource Constraint in the Small Island Developing States: Essays in Entrepreneurial Cognition MGSoG Dissertations Series, № 72

\section{Cécile Cherrier}

The Expansion of Basic Social Protection in Low-income Countries: An Analysis of Foreign Aid Actors' Role in the Emergence of Social Transfers in Sub-Saharan Africa MGSoG Dissertations series, № 71 


\section{Paul Caldron}

The Tacit Bargain in Short-Term

Medical Missions: Why U.S.

physicians go and what it costs

MGSoG Dissertation Series, № 70

\section{Mahmut Kobal}

Customs \& Excellence: A Comparative Approach on Administrative and

Regulatory Compliance Perspectives of the EU-Turkey Customs Union

MGSoG Dissertation Series, № 69

\section{Craig Loschmann}

Essays on Conflict-related Migration

and Development in the Case of

Afghanistan

MGSoG Dissertations Series, № 68

\section{Andrea Milan}

Rural Livelihoods, Location and

Vulnerable Environments: Approaches to Migration in Mountain areas of

Latin America

MGSoG Dissertation Series, № 67

\section{Farida Lada}

On Guarding the Welfare of Clinical

Trial Subjects While Promoting Novel

Drug Innovation

A Game Theoretical Approach

MGSoG Dissertation Series, № 66

\section{5}

\section{Hibret Belete Maemir}

Dissecting Aggregate Productivity:

International Integration and Growth

with Heterogeneous Firms

UNU-MERIT Dissertation Series, № 96

\section{Giorgio Triulzi}

Looking for the Right Path: Technology

Dynamics, Inventive Strategies and

Catching-up in the Semiconductor

Industry

UNU-MERIT Dissertation Series, № 95

\begin{abstract}
Abdul Baseer Qazi
Knowledge flows and networks in the ICT sector: The case of Pakistan

UNU-MERIT Dissertation Series, № 94
\end{abstract}

\section{Ajay Thutupalli \\ Technology Paradigm Shifts in Agriculture: Drivers of Sustainability and Catch up \\ UNU-MERIT Dissertation Series, № 93}

\section{Eduardo Urias}

Improving access to HIVIAIDS

treatment in Brazil: When are

Compulsory Licenses effective in Price Negotiations?

UNU-MERIT Dissertation Series, № 92

\section{Francesca Guadagno}

Why have so few Countries

Industrialised?

UNU-MERIT Dissertation Series, № 91

\section{Daniel Opolot}

The Evolution of Beliefs and Strategic

Behaviour

UNU-MERIT Dissertation Series, № 90 


\section{Alejandro Lavopa}

Structural Transformation and

Economic Development: Can

Development Traps be Avoided

UNU-MERIT Dissertation Series, № 89

\section{Jinjin Zhao}

Urban water management reform: The

Case of China

UNU-MERIT Dissertation Series, № 88

\section{Simona Vezzoli}

Borders, Independence and Postcolonial Ties: the Role of the State in

Caribbean Migration

MGSoG Dissertation Series, № 65

\section{Silvia Consuelo Gómez Soler}

Civil Conflict and Education: How

Does Exposure to Civil Conflict Affect

Human Capital Accumulation?

Evidence from Standardized Exit

Exams in Colombia

MGSoG Dissertation Series, № 64

\section{Paula Nagler}

Occupational Choice in the Developing

World

MGSoG Dissertation Series, № 63

\section{Jasmin Kientzel}

Determinants of Professional

Commitment to Environmental

Sustainability

MGSoG Dissertation Series, № 62

\section{Mehmet Güney Celbiş}

Regional Policies: Convergence, Trade, and the Allocation of Public Capital MGSoG Dissertation Series, № 61

\section{Florian Henning}

Living Up to Standard:

Interoperability Governance and

Standards Adoption in Government

Information Networks

MGSoG Dissertation Series, № 60

\section{Niels P. Groen}

The Never-Ending Project

Understanding E-Government Project

Escalation

MGSoG Dissertation Series, № 59

\section{Derek Copp}

Teacher-Based Reactivity to Provincial

Large-scale Assessment in Canada

MGSoG Dissertation Series, № 58

\section{Michaella Vanore}

Family-Member Migration and the

Psychosocial Health Outcomes of

Children in Moldova and Georgia

MGSoG Dissertation Series, № 57

\section{Sonja Fransen}

The Economic and Social Effects of

Remittances and Return Migration in

Conflict-Affected Areas: The Case of

Burundi

MGSoG Dissertation Series, № 56

\section{Ibrahim Khalil Conteh}

The Impact of Floods on Primary

School Education in Zambia

MGSoG Dissertation Series, № 55 


\section{Richard Bluhm}

Growth Dynamics and Development Essays in Applied Econometrics and Political Economy

MGSoG Dissertation Series, № 54

\section{Nevena P. Zhelyazkova}

Work-Family Reconciliation and Use of Parental Leave in Luxembourg:

Empirical Analysis of Administrative Records

MGSoG Dissertation Series, № 53

2014

\section{Dirk Crass}

The Impact of Brands on Innovation and Firm Performance: Empirical Evidence from Germany UNU-MERIT Dissertation Series, № 87

\section{Samyukta Bhupatiraju}

The Geographic Dimensions of Growth and Development

UNU-MERIT Dissertation Series, № 86

\section{François Lafond}

TheEvolution of Knowledge Systems UNU-MERIT Dissertation Series, № 85

\section{Annalisa Primi}

Promoting Innovation in Latin America: What Countries Have Learned (and What They Have Not) in Designing and Implementing Innovation and Intellectual Property Policies

UNU-MERIT Dissertation Series, № 84

\section{Fatoumata Lamarana Diallo}

Evaluation of Meal and Deworming Programs for Primary Schools in Rural Senegal

UNU-MERIT Dissertation Series, № 83

\section{Sachin Kumar Badkas}

Metachoice and Metadata: Innovating with Environmental Policy Analysis in Europe

MGSoG Dissertation Series, № 52

\section{Irina S. Burlacu}

An Evaluation of Tax-Benefit Systems Impact on the Welfare of Frontier

Worker:

The Case of Luxembourg and Belgium MGSoG Dissertation Series, № 51

\section{Özge Bilgili}

Simultaneity in Transnational Migration Research: Links Between Migrants' Host and Home Country

Orientation

MGSoG Dissertation Series, № 50 


\section{Yulia Privalova Krieger}

Reshaping the Big Agenda:

Transnational Politics and Domestic

ResistanceFinancial crisis and social

protection reform in Bosnia and

Herzegovina

MGSoG Dissertation Series, № 49

\section{Marieke van Houte}

Moving Back or Moving Forward?

Return migration after Conflict

MGSoG Dissertation Series, № 48

\section{Oxana Slobozhan}

Global Governance in the Management of Natural Resources: The Case of the

Extractive Industries Transparency

Initiative (EITI)

MGSoG Dissertation Series, № 47

\section{Luis Bernardo Mejia Guinand}

The Changing Role of the Central

Planning Offices in Latin America: A

Comparative Historical Analysis

Perspective (1950-2013)

MGSoG Dissertation Series, № 46

\section{Cheng Boon Ong}

Ethnic Segregation in Housing,

Schools and Neighbourhoods in the

Netherlands

MGSoG Dissertation Series, № 45

\section{Luciana V. Cingolani}

Bureaucracies for Development:

Oxymoron or Reality? Studies on State

Capacity in Challenging Governance

Contexts

MGSoG Dissertation Series, № 44

\section{Carlos Cadena Gaitán}

Green Politics in Latin American

Cities - Sustainable Transport Agendas

MGSoG Dissertation Series, № 43

\section{Katie Kuschminder}

Female Return Migration and

Reintegration Strategies in Ethiopia

MGSoG Dissertation Series, № 42

\section{Metka Hercog}

Highly-Skilled Migration and New

Destination Countries

MGSoG Dissertation Series, № 41

\section{Margaret Agaba Rugadya}

Can Remittances Influence the Tenure

and Quality of Housing in Uganda?

MGSoG Dissertation Series, № 40

\section{Ilire Agimi}

New Governance Under Limited

Statehood: The Case of Local

Government Reform in Kosovo

MGSoG Dissertation Series, № 39

\section{3}

\author{
Anant Kamath \\ Information Sharing through Informal \\ Interaction in Low-Tech Clusters \\ UNU-MERIT Dissertation Series, \\ № 82
}




\section{Flavia Pereira de Carvalho}

What we talk about when we talk about

Brazilian Multinationals: An

Investigation on Brazilian FDI,

Economic Structure, Innovation and

the Relationship between them

UNU-MERIT Dissertation Series, № 81

\section{Jun Hou}

Complementarity in Innovation and

Development: A Cross-country

Comparison

UNU-MERIT Dissertation Series, № 80

\section{Rufin Baghana}

Impacts of Government Incentives to $R \mathcal{E D}$, Innovation and Productivity:

A Microeconometric Analysis of the

Québec Case

UNU-MERIT Dissertation Series, № 79

\section{Lilia I. Stubrin}

High-Tech Activities in Emerging

Countries: A Network perspective on

the Argentinean Biotech Activity

UNU-MERIT/MGSoG Dissertation

Series, № 78

\section{Kristine Farla}

Empirical Studies on Institutions,

Policies and Economic Development

MGSoG Dissertation Series, № 38

\section{Marina Petrovic}

Social Assistance and Activation in the Pursuit of Happiness: Shedding New

Light on Old Policy Solutions to Social Exclusion

MGSoG Dissertation Series, № 37

\section{Laura Torvinen}

Assessing Governance Assessments:

The Case of Mozambique: Governance

Assessments in the Context of Aid

Effectiveness Discourse

MGSoG Dissertation Series, № 36

\section{Biniam Egu Bedasso}

Institutional Change in the Long

Shadow of Elite: Essays on

Institutions, Human Capital and

Ethnicity in Developing Countries

MGSoG Dissertation Series, № 35

\section{Sepideh Yousefzadeh Faal}

Deghati

Childhoods Embargoed: Constructing and Reconstructing Multidimensional

Child Poverty in Iran 1984-2009

MGSoG Dissertation Series, № 34

\section{Robert Bauchmüller}

Investing in Early Childhood Care and Education: The Impact of Quality on Inequality

MGSoG Dissertation Series, № 33

\section{Martin Rehm}

Unified Yet Separated: Empirical

Study on the Impact of Hierarchical

Positions within Communities of

Learning

MGSoG Dissertation Series, № 32 


\section{Abdul Waheed}

Innovation Determinants and

Innovation as a Determinant: Evidence

from Developing Countries

UNU-MERIT Dissertation Series,

№ 77

\section{Bilal Mirza}

Energy Poverty and Rural Energy

Markets in Pakistan

UNU-MERIT Dissertation Series, № 76

\section{Benjamin Engelstätter}

Enterprise Software and Video Games:

An Empirical Analysis

UNU-MERIT Dissertation Series, № 75

\section{Fulvia Farinelli}

Natural Resources, Innovation and

Export Growth: The Wine Industry in

Chili and Argentina

UNU-MERIT Dissertation Series

\section{Rodolfo Lauterbach}

Innovation in Manufacturing: From

Product Variety and Labor

Productivity Growth to Economic

Development in Chile

UNU-MERIT Dissertation Series

\section{Kirsten Wiebe}

Quantitative Assessment of

Sustainable Development and Growth

in Sub-Saharan Africa

UNU-MERIT Dissertation Series, № 74
Julio Miguel Rosa

Organizational Strategies, Firms'

Performance and Spatial Spillovers:

The Canadian Case in Research and

Development.

UNU-MERIT Dissertation Series, № 73

Johannes Wilhelmus Marie Boels

Joseph Schumpeter, Honderd Jaar

Economische Ontwikkeling: Een

Historisch-theoretische Beschouwing.

UNU-MERIT Dissertation Series

\section{Dorcas Mbuvi}

Utility Reforms and Performance of the

Urban Water Sector in Africa

MGSoG Dissertation Series, № 31

\section{Lina Salanauskaite}

Distributional Impacts of Public

Policies: Essays in Ex-Ante and Ex-

Post Evaluation

MGSoG Dissertation Series, № 30

\section{Esther Schüring}

To Condition or not - is that the

Question?

An Analysis of the Effectiveness of Ex-

Ante and Ex-Post Conditionality in

Social Cash Transfer Programs

MGSoG Dissertation Series, № 29

\section{Joe Abah}

Strong Organisations in Weak States:

Atypical Public Sector Performance in

Dysfunctional Environments

MGSoG Dissertation Series, № 28 


\section{Zina Samih Nimeh}

Social Citizenship Rights: Inequality and Exclusion

MGSoG Dissertation Series, № 27

2011

\section{Daniel Vertesy}

Interrupted Innovation: Emerging

Economies in the Structure of the

Global Aerospace Industry

UNU-MERIT Dissertation Series, № 72

\section{Tina Saebi}

Successfully Managing Alliance

Portfolios: AnAlliance Capability View UNU-MERIT Dissertation Series, № 71

\section{Nora Engel}

Tuberculosis in India: A Case of

Innovation and Control

UNU-MERIT/MGSoG Dissertation

Series, № 70

\section{Evans Mupela}

Connectivity and growth in Sub-

Saharan Africa: The Role of

Communication Satellites

UNU-MERIT Dissertation Series, № 69

\section{Nantawan Kwanjai}

Cross Cultural Intelligence amid Intricate Cultural Webs: A Tale of the UnDutchables in the Land of 1002 Smiles

UNU-MERIT Dissertation Series, № 68

\section{Lina Sonne}

Innovation in Finance to Finance Innovation: Supporting Pro-poor Entrepreneur-based Innovation UNU-MERIT Dissertation Series, № 67

\section{Lenka Eisenhamerová}

Legitimacy of 'Humanitarian Military Intervention'

MGSoG Dissertation Series, № 26

\section{Sonila Tomini}

Informal Payments for Health Care

Services in Albania

MGSoG Dissertation Series, № 25

\section{Jinjing Li}

Dynamic Microsimulation in Public

Policy Evaluation

MGSoG Dissertation Series, № 24

\section{Aziz Atamanov}

Rural Nonfarm Employment and International Migration as

Alternatives to Agricultural

Employment: The Case of Kyrgyzstan

MGSoG Dissertation Series, № 23

\section{Frieda Vandeninden}

Poverty Alleviation: Aid and Social

Pensions

MGSoG Dissertation Series, № 22

\section{Juliana Nyasha Tirivayi}

The Welfare Effects of Integrating AIDS Treatment with Food Transfers: Evidence from Zambia MGSoG Dissertation Series, № 21 
Agnieska Ewa Sowa

Who's Left Behind? Social Dimensions

of Health Transition and Utilization of

Medical Care in Poland

MGSoG Dissertation Series, № 20

\section{Emmanaouil Sfakianakis}

The Role of Private Actors in the

Provision of Public Goods with

Applications to Infrastructure and

Financial Stability

MGSoG Dissertation Series, № 19

\section{Siu Hing Lo}

White Collars Green Sleeves: An Inter-

organizational Comparison of

Determinants of Energy-Related

Behaviors among Office Workers

MGSoG Dissertation Series, № 18

\section{Treena $\mathbf{W u}$}

Constraints to Human Capital

Investment in Developing Countries:

Using the Asian Financial Crisis in

Indonesia as a Natural Experiment

MGSoG Dissertation Series, № 17

\section{Henry Espinoza Peña}

Impact Evaluation of a Job-Training

Programme for Disadvantaged Youths:

The Case of Projoven

MGSoG Dissertation Series, № 16
2010

Fernando Santiago

Human Resources Management

Practices and Learning for Innovation in Developing Countries:

Pharmaceutical Firms in Mexico

UNU-MERIT Dissertation Series,

№ 66

\section{Zakaria Babutsidze}

Essays on Economies with

Heterogeneous Interacting Consumers

UNU-MERIT Dissertation Series,

№ 65

\section{Bertha Vallejo}

Learning and Innovation Under

Changing Market Conditions: The

Auto Parts Industry in Mexico

UNU-MERIT Dissertation Series,

№ 64

\section{Donatus Ayitey}

Technical Change, Competitiveness

and Poverty Reduction: A Study of the

Ghanaian Apparel Industry

UNU-MERIT Dissertation Series,

№ 63

\section{Sergey Filippov}

Multinational Subsidiary Evolution:

Corporate Change in New EU Member

States

UNU-MERIT Dissertation Series, № 62 


\section{Asel Doranova}

Technology Transfer and Learning under the Kyoto Regime: Exploring the Technological Impact of CDM Projects in Developing Countries

UNU-MERIT Dissertation Series, № 61

\section{Florian Tomini}

Between Family and Friend:

Understanding the Interdependency of Private Transfers

MGSoG Dissertation Series, № 15

\section{Michał Polalowski}

The Institutional Transformation of

Social Policy in East Central Europe:

Poland and Hungary in Comparative and Historical Perspective

MGSoG Dissertation Series, № 14

\section{Maha Ahmed}

Defining, Measuring and Addressing Vulnerability: The Case of Post

Conflict Environments

MGSoG Dissertation Series, № 13

\section{Pascal Beckers}

Local Space and Economic Success:

The Role of Spatial Segregation of

Migrants in the Netherlands

MGSoG Dissertation Series, № 12

\section{Victor Cebotari}

Conflicting Demands in Ethnically

Diverse Societies: Ethno political

Contention and Identity Values in

Europe

MGSoG Dissertation Series, № 11

\section{Dennis Gyllensporre}

Competing and Complementary

Perspectives on the EU as a Crisis

Management Actor:

An Examination of the Common

Security and Defence Policy through

the Lenses of Idealism and Realism

MGSoG Dissertation Series, № 10

\section{Judit Vall Castello}

Business Cycle and Policy Effects on Labour Market Transitions of Older and Disabled Workers in Spain

MGSoG Dissertation Series, № 9

\section{Keetie Roelen}

False Positives or Hidden Dimensions:

The Definition and Measurement of

Child Poverty

MGSoG Dissertation Series, № 8

Denisa Maria Sologon

Earning Dynamics in Europe

MGSoG Dissertation Series, № 7

\section{Melissa Siegel}

Money and Mobility: Migration and

Remittances

MGSoG Dissertation Series, № 6

Jessica S. Hagen-Zanker

Modest Expectations: Causes and

Effects of Migration on Migrant

Households inSource Countries

MGSoG Dissertation Series, № 5 
Alexis Habiyaremye

From Primary Commodity Dependence to Diversification and Growth:

Absorptive Capacity and Technological Catch Up in Botswana and Mauritius. UNU-MERIT Dissertation Series, № 60

\section{Yoseph Getachew}

The Role of Public Capital in Economic Development

UNU-MERIT Dissertation Series, № 59

\section{Sandra Leitner}

Embodied Technological Change and Patterns of Investment in Austrian Manufacturing UNU-MERIT Dissertation Series, № 58

\section{Semih Akçomak}

The Impact of Social Capital on Economic and Social Outcomes UNU-MERIT Dissertation Series, № 57

\section{Abraham Garcia}

The Role of Demand in Technical Change

UNU-MERIT Dissertation Series, № 56

\section{Saurabh Arora}

Coherence in Socio-technical Systems: A Network Perspective on the Innovation Process UNU-MERIT Dissertation Series, № 55
Human Development and Autonomy in Project Aid: Experiences from four bilateral projects in Nicaragua and $E l$ Salvador

MGSoG Dissertation Series, № 4

\section{Christiane Arndt}

Governance Indicators

MGSoG Dissertation Series, № 3

\section{Britta Augsburg}

Microfinance: Greater Good or Lesser Evil?

MGSoG Dissertation Series, № 2

\section{8}

\section{Rutger Daems}

Medicines for the Developing World UNU-MERIT Dissertation Series, № 54

\section{Johannes Hanel}

Assessing Induced Technology:

Sombart's Understanding of Technical

Change in the History of Economics

UNU-MERIT Dissertation Series, № 53

\section{Rifka Weehuizen}

Mental Capital: the Economic Significance of Mental Health UNU-MERIT Dissertation Series, № 52 


\section{Danielle Cloodt}

The Relationship between RED

Partnership Formation, Social

Embeddedness and Innovative

Performance

UNU-MERIT Dissertation Series, № 51

\section{Sabine Fuss}

Sustainable Energy Development

under Uncertainty

UNU-MERIT Dissertation Series, № 50

\section{Geranda Notten}

Measuring and Managing Poverty

Risks

MGSoG Dissertation Series, № 1

2007

\section{Tobias Kronenberg}

Reconciling Environmental

Conservation with Economic

Prosperity: The Feasibility of Double

Dividends in the Short and Long Run

UNU-MERIT Dissertation Series, № 49

\section{Viktoria Kravtsova}

Assessing the Impact of Foreign Direct Investment in Transition Economies UNU-MERIT Dissertation Series, № 48

\section{Suhail Sultan}

The Competitive Advantage of Small and Medium Sized Enterprises: The Case of Jordan's Natural Stone Industry UNU-MERIT Dissertation Series, № 47

\section{6}

\section{Bulat Sanditov}

Essays on Social Learning and Imitation

UNU-MERIT Dissertation Series, № 46

\section{Mamata Parhi}

Dynamics of New Technology Diffusion: A Study of the Indian Automotive Industry

UNU-MERIT Dissertation Series, № 45

\section{Andreas Reinstaller}

Social Structures and the Innovation Process: Their Role in the Demand of Firms and Consumers

UNU-MERIT Dissertation Series, № 44

\section{Rose Kiggundu}

Innovation systems and Development: The Journey of a Beleaguered Nile Perch Fishery in Uganda UNU-MERIT Dissertation Series, № 43 
Thomas Pogue

The Evolution of Research

Collaboration in South African Gold

Mining: 1886-1933

UNU-MERIT Dissertation Series, № 42

\section{Geoffrey Gachino}

Foreign Direct Investment, Spillovers and Innovation: The Case of Kenyan

Manufacturing Industry

UNU-MERIT Dissertation Series, № 41

\section{Önder Nomaler}

Technological Change, International

Trade and Growth: An Evolutionary,

Multi-Agents-Based Modeling

Approach

UNU-MERIT Dissertation Series, № 40

2005

Samia Satti Osman Mohamed-

Nour

Change and Skill Development in the Arab Gulf Countries

UNU-MERIT Dissertation Series, № 39

\section{Elad Harison}

Intellectual Property Rights:

Economics and Policy Analysis

UNU-MERIT Dissertation Series, № 38

\section{Daniel Dalohoun}

The Relationship between RED

Partnership Formation, Social

Embeddedness and Innovative

Performance: a Multi-level Approach

of Social Embeddedness

UNU-MERIT Dissertation Series, № 37

\section{Müge Ozman}

Networks, Organizations and

Knowledge

UNU-MERIT Dissertation Series,

№ 36

\section{Bas Straathof}

Product Variety and Economic

Growth: The Counteracting Effects of

Scale and Idiosyncrasy

UNU-MERIT Dissertation Series, № 35

\section{Wilfred Schoenmakers}

Knowledge Flows between

Multinational Companies: A Patent

Data Analysis

UNU-MERIT Dissertation Series,

№ 34

\section{Myriam Cloodt}

Mergers and Acquisitions ( $M$ and As) in High-Tech Industries: Measuring the Post-M and A Innovative Performance of Companies UNU-MERIT Dissertation Series, № 33 
2004

\section{Paola Criscuolo}

$R \mathcal{E D}$ Internationalisation and

Knowledge Transfer: Impact on MNEs and their Home Countries

UNU-MERIT Dissertation Series, № 32

\section{Maarten Verkerk}

Trust and Power on the Shop Floor UNU-MERIT Dissertation Series, № 31

\section{Gottfried Leibbrandt}

Adoption, Harmonization and

Succession of Network Technologies across Countries

UNU-MERIT Dissertation Series, № 30

\section{Mark Sanders}

Skill Biased Technical change: Its Origins, the Interaction with the Labour Market and Policy Implications UNU-MERIT Dissertation Series, № 29

2003

\section{Nadine Roijakkers}

Inter-firm Cooperation in High-tech Industries: a Study of RED

Partnerships in Pharmaceutical

Biotechnology

UNU-MERIT Dissertation Series, № 28
Viki Sonntag

Speed, Scale and Sustainability

UNU-MERIT Dissertation Series, № 27

\section{Masaru Yarime}

From End-of-Pipe Technology to Clean Technology

UNU-MERIT Dissertation Series, № 26

\section{Stéphane Malo}

The Combinatorial Chemistry

Revolution: Sustaining a Superior

Performance Position through

Technological Learning

UNU-MERIT Dissertation Series, № 25

2002

\section{Annelies Hogenbirk}

Determinants of Inward Foreign Direct Investment: the Case of the Netherlands

UNU-MERIT Dissertation Series, № 24

\section{Bastiaan Johan terWeel}

The Computerization of the Labour Market

UNU-MERIT Dissertation Series 


\section{Marjolein Caniëls}

\section{John Adeoti}

Technology Investment in Pollution Control in Sub-Saharan Africa: The Case of the Nigerian Manufacturing Industry

UNU-MERIT Dissertation Series, № 23

\section{Edward Huizenga}

Innovation Management: How

Frontrunners Stay Ahead: An

Empirical Study on Key Success

Factors in the ICT sector

UNU-MERIT Dissertation Series, № 22

2000

\section{Machiel van Dijk}

Technological Change and the

Dynamics of Industries: Theoretical Issues and Empirical evidence from Dutch Manufacturing UNU-MERIT Dissertation Series, № 21

\section{9}

\section{Jan Cobbenhagen}

Managing Innovation at the Company Level: A Study on Non-Sector-Specific Success Factors UNU-MERIT Dissertation Series, № 20
Regional Growth Differentials: The Impact of Locally Bounded Knowledge Spillovers

UNU-MERIT Dissertation Series, № 19

\section{8}

\author{
Aldo Geuna \\ Resource Allocation and Knowledge \\ production: Studies in the Economics \\ of University Research \\ UNU-MERIT Dissertation Series, \\ № 18 \\ 1996 \\ Reinoud Joosten \\ Dynamics, Equilibria, and Values \\ UNU-MERIT Dissertation Series, \\ № 17
}

\section{Hugo Kruiniger}

Investment, $R \mathcal{E} D$, and the Financing Decisions of the Firm

UNU-MERIT Dissertation Series, № 16

\section{5}

\section{Hans van Meijl}

Endogenous Technological Change: The Case of Information Technology, Theoretical Considerations and Empirical Results UNU-MERIT Dissertation Series, № 15 


\section{René Kemp}

Environmental Policy and Technical

Change: A Comparison of the

Technological Impact of Policy

Instruments

UNU-MERIT Dissertation Series, № 14

\section{Rohini Acharya}

The Impact of New Technologies on

Economic Growth and Trade: A Case

Study of Biotechnology

UNU-MERIT Dissertation Series, № 13

\section{Geert Duysters}

The Evolution of Complex Industrial Systems: The Dynamics of Major IT Sectors

UNU-MERIT Dissertation Series, № 12

\section{Marjan Groen}

Technology, Work and Organisation: A Study of the Nursing Process in Intensive Care Units

UNU-MERIT Dissertation Series, № 11

1994

\section{Huub Meijers}

On the Diffusion of Technologies in a Vintage Framework: Theoretical Considerations and Empirical Results UNU-MERIT Dissertation Series, № 10

\section{Theon van Dijk}

The Limits of Patent Protection: Essays on the Economics of Intellectual

Property Rights

UNU-MERIT Dissertation Series, № 9

\section{Hans Voordijk}

Naar Integrale Logistiek in

Bedrijfsketens: Ontwikkelingen in de Bouw

UNU-MERIT Dissertation Series, № 8

1993

\section{Paul Diederen}

Technological Progress in Enterprises and Diffusion of Innovation:

Theoretical Reflections and Empirical Evidence

UNU-MERIT Dissertation Series, № 7

\section{Ben Dankbaar}

Economic Crisis and Institutional Change: The Crisis of Fordism from the Perspective of the Automobile Industry UNU-MERIT Dissertation Series, № 6

\section{Hanno Roberts}

Accountability and Responsibility: The Influence of Organisation Design on Management Accounting UNU-MERIT Dissertation Series, № 5 


\section{Bart Verspagen}

Uneven Growth between

Interdependent Economies: An

Evolutionary View on Technology

Gaps, Trade and Growth

UNU-MERIT Dissertation Series,

№ 4

\section{Sjoerd Romme}

A Self-organization Perspective on

Strategy Formation

UNU-MERIT Dissertation Series,

№ 3

1989

John Spangenberg

Economies of Scale, and Atmosphere in

Research Organisations

UNU-MERIT Dissertation Series,

№ 2

1988

John Hagedoorn

Evolutionary and Heterodox

Innovation Analysis: A Study of

Industrial and Technological

Development in Process Control and

Information Technology

UNU-MERIT Dissertation Series, № 1 
